University of Redlands

\title{
A Cartographic Standard for Hopi Archaeological Sites
}

\author{
A Major Individual Project submitted in partial satisfaction of the requirements \\ for the degree of Master of Science in Geographic Information Systems \\ by \\ Brittany Linnea Miller \\ Douglas Flewelling, Ph.D., Committee Chair \\ Nader Afzalan, Ph.D.
}

August 2016 
A Cartographic Standard for Hopi Archaeological Sites

Copyright (C) 2016

by

Brittany Linnea Miller 
The report of Brittany Linnea Miller is approved.
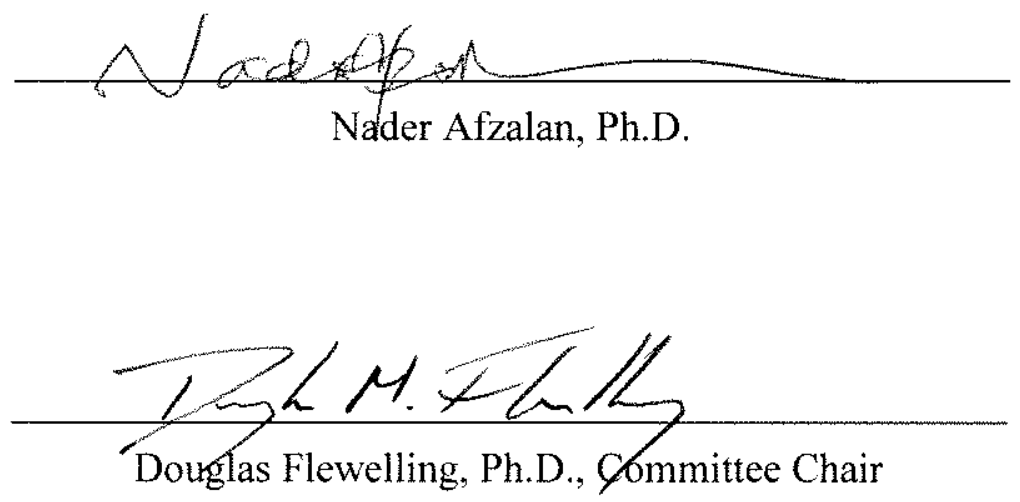

August 2016 



\section{Acknowledgements}

I owe a great deal of gratitude to those who made this thesis possible, not only for their insight throughout the project but also for making my experience a memorable one. First, I want to thank my advisors, Dr. Douglas Flewelling and Dr. Mark Kumler, for their time and patience while working with me, as well as Dr. Nader Afzalan for serving on my committee. Their unyielding encouragement and criticism helped me explore new avenues to address some of the most challenging aspects of this project. I will also like to thank all the Spatial Studies faculty and staff at the University of Redlands for providing invaluable knowledge and helping me navigate throughout the program. I must also express my gratitude to Dr. Wesley Bernardini who made choosing this project possible. As my client, he provided valuable feedback and resources as I worked on the project and was patient even when certain tasks took longer than anticipated.

Also, thank you to Cohort 28 for their support, friendship, and sporadic adventures that I will continue to cherish. I hope that the friendships we made this past year will continue to grow. Lastly, and with the sincerest gratitude, I want to acknowledge my parents, brother, and fiancé for all of their love and faith in me, not only in the past year but also for all the years together. They provided the basis for my success as an individual and give reason to my accomplishments, and without them, enduring this year may not have been possible. 



\begin{abstract}
A Cartographic Standard for Hopi Archaeological Sites
\end{abstract}

by

Brittany Linnea Miller

Many archaeologists use geographic information systems (GIS) to visualize existing excavation sites and predict new site locations. Maps depict much of this information, and though researchers conduct a significant amount of research on GIS in archaeology, few focus on the cartographic aspect of archaeological maps. Prior to GIS, archaeologists tediously hand-drew maps using various cartographic techniques, but many of the unique design elements found in archaeological maps have been lost in recent years. With a plethora of symbol choices contained in many GIS interfaces, it is difficult to remember that consistency and simplicity is vital for effective communication. This project improved archaeological efforts on the Hopi reservation in Arizona by establishing a standardized approach of representing spatial fieldwork data. The results included a geodatabase that standardized the projection system of the input data, a custom symbol set depicting archaeological features tested through an online survey, a map series using the custom symbology, and three-dimensional models of the major excavation sites. 



\section{Table of Contents}

Chapter 1 - Introduction ................................................................................................ 1

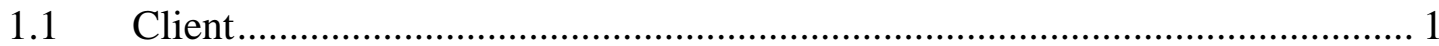

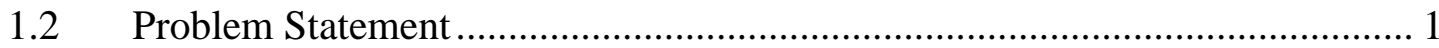

$1.3 \quad$ Proposed Solution ............................................................................... 1

1.3.1 Goals and Objectives ........................................................................... 2

1.3.2 Scope

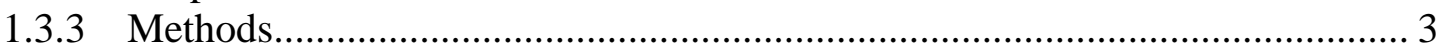

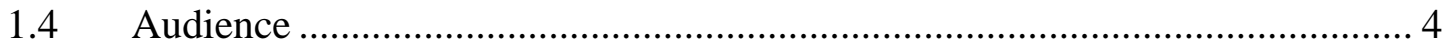

1.5 Overview of the Rest of this Report .......................................................... 4

Chapter 2 - Background and Literature Review ............................................................. 5

$2.1 \quad$ Overview of Cartographic Principles.......................................................... 5

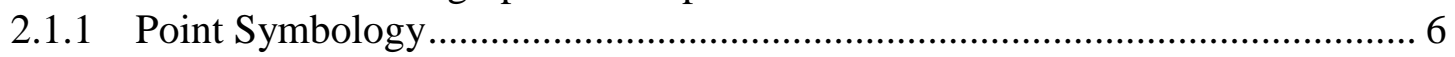

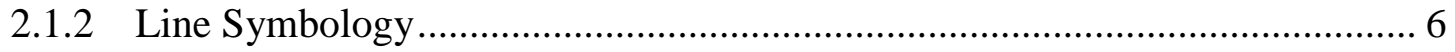

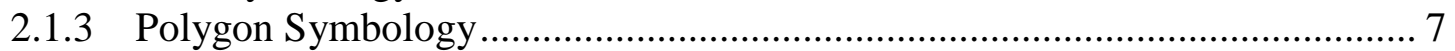

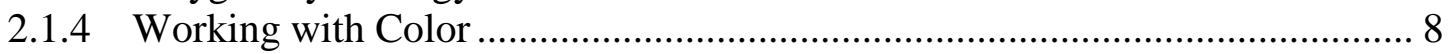

2.1.5 Text and Labeling .............................................................................. 9

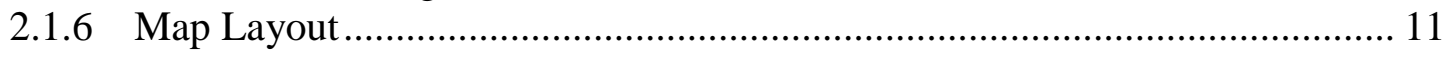

2.2 Cartographic Designs in Archaeology ………………………………........ 11

2.2.1 Current Archaeological Symbol Sets ........................................................... 12

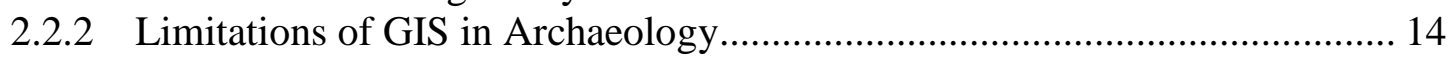

2.3 Modeling Archaeological Sites............................................................. 16

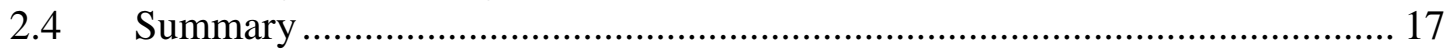

Chapter 3 - Systems Analysis and Design....................................................................... 19

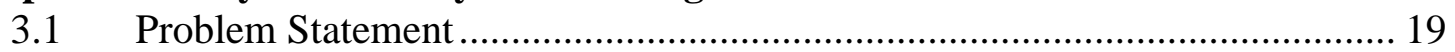

3.2 Requirements Analysis ………………………….................................... 20

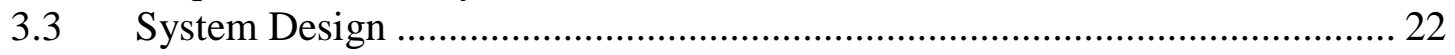

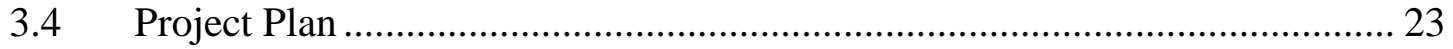

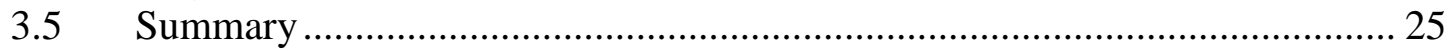

Chapter 4 - Database Design............................................................................................... 27

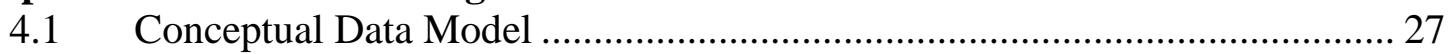

4.2 Logical Data Model ……………......................................................... 29

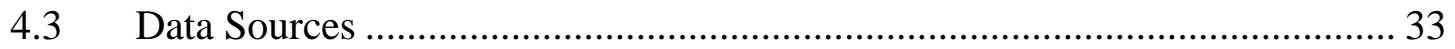

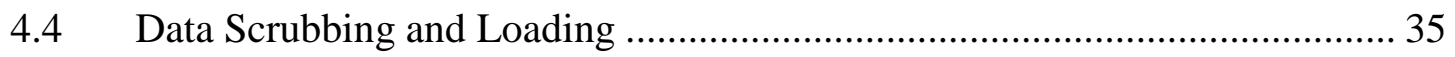

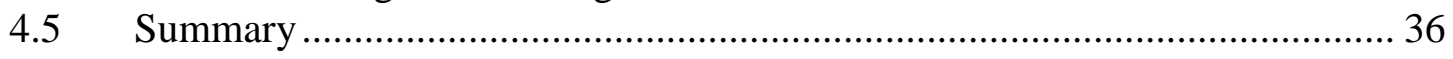

Chapter 5 - Implementation.................................................................................39

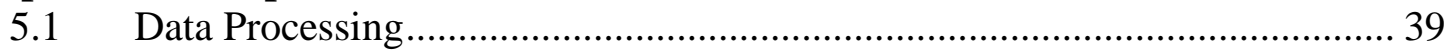

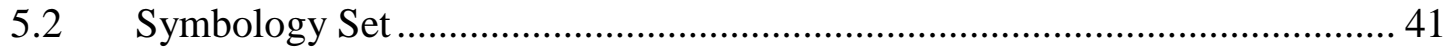

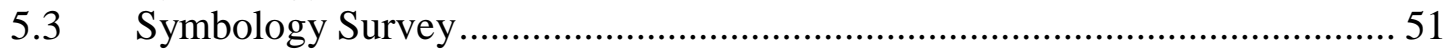

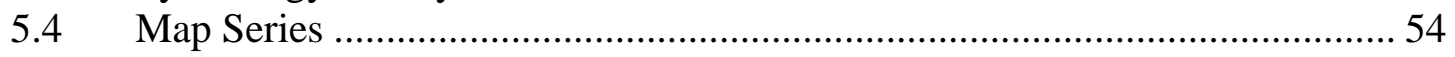

5.5 Three Dimensional Models....................................................................... 57

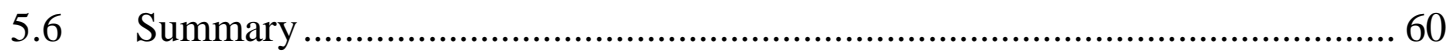


Chapter 6 - Results and Analysis............................................................................................ 63

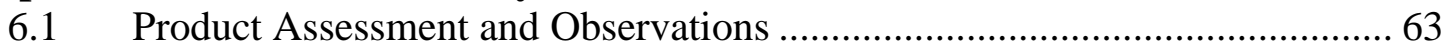

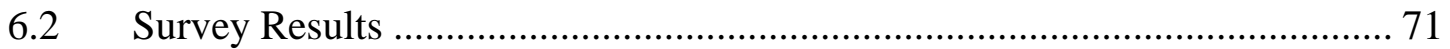

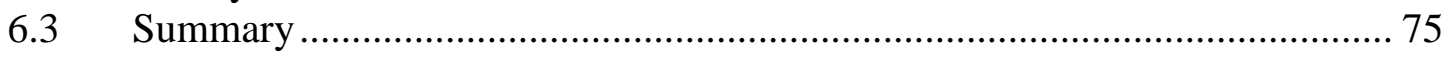

Chapter 7 - Conclusions and Future Work .............................................................. 77

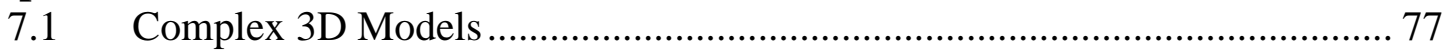

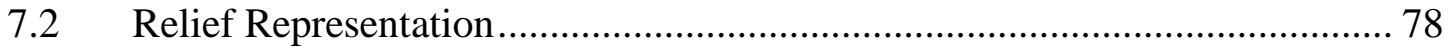

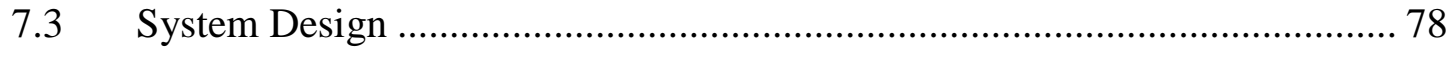

Works Cited …...................................................................................................................... 79

Appendix A. Map Series................................................................................................. 81

Appendix B. Symbology Catalog................................................................................. 226

Appendix C. Survey Results ................................................................................................. 227

Appendix D. Python Script .................................................................................................. 252 


\section{Table of Figures}

Figure 1-1: Archaeology Sites on the Hopi Reservation. ...................................... 2

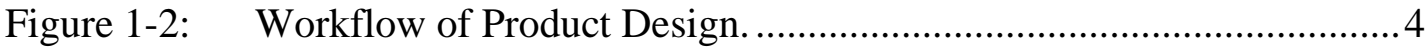

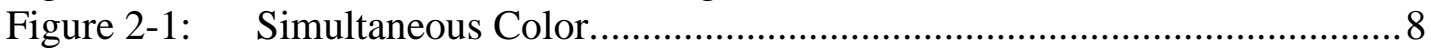

Figure 2-2: $\quad$ Labeling Hierarchy and Conventions. ............................................ 10

Figure 2-3: $\quad$ Four Observed Archaeological Symbol Sets. .................................... 14

Figure 2-4: Traditional Archaeological Map....................................................... 15

Figure 3-1: $\quad$ Original Map Designs Using the Asterisk Symbol Inconsistently. ... 20

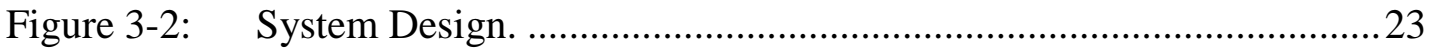

Figure 3-3: $\quad$ Original and Revised Project Plan Comparison..................................25

Figure 4-1: Conceptual Data Model. .................................................................28

Figure 4-2: Contents Diagram of Sites within the Hopi Reservation..................29

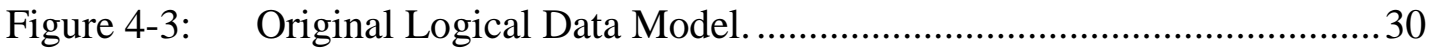

Figure 4-4: Attribute Schema Inconsistencies for Collection Unit Data...............32

Figure 4-5: $\quad$ Revised Logical Data Model. ............................................................ 33

Figure 4-6: Imagery Requested on Image Finder from Digital Globe Foundation.

World View 2 satellite 50cm resolution imagery (2010-2015)........ 34

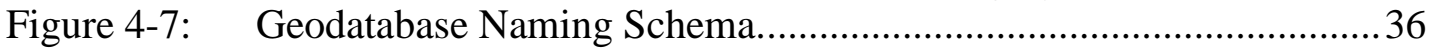

Figure 5-1: $\quad$ Position Inaccuracy after Projecting Input Data..............................40

Figure 5-2: Workflow for Merging the Mungyaovi Feature Dataset. ................... 41

Figure 5-3: $\quad$ Workflow for Creating the Hopi Archaeology Set Symbology......... 44

Figure 5-4: Marker Symbols in the Hopi Archaeology Set. ................................ 45

Figure 5-5: $\quad$ Adjusted Marker Symbols to Represent Point of Origin. ....................46

Figure 5-6: Polygon Fills in the Hopi Archaeology Set. .....................................47

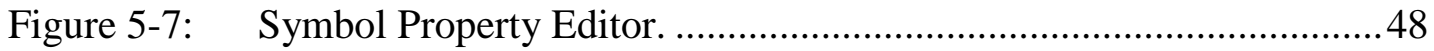

Figure 5-8: $\quad$ Line Symbols in the Hopi Archaeology Set ....................................49

Figure 5-9: Hopi Archaeology Set within the Style Manager ..............................50

Figure 5-10: Symbol Selector Interface ............................................................. 51

Figure 5-11: Samples of the Survey.....................................................................53

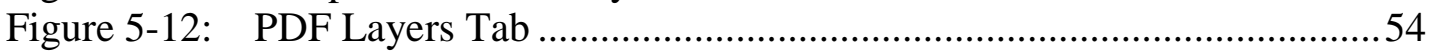

Figure 5-13: Maplex Labeling Interface ..........................................................55

Figure 5-14: Comparison between Wall and Inferred Wall Symbology ................56

Figure 5-15: Sketch Properties to Flip Vertices on Cliff Edge Data .......................57

Figure 5-16: Digital Globe Imagery overlay in ArcScene 10.3 ............................58

Figure 5-17: Digital Globe Imagery overlay in ArcGIS Pro 1.2 ..........................59

Figure 5-18: Symbology for 3D Models in Arc GIS Pro 1.2 ...............................60

Figure 6-1: $\quad$ Original Map Layout (left) and Final Map Layout (right) Examples . 64

Figure 6-2: Wall and Inferred Wall Symbology at Different Scales. ...................65

Figure 6-3: Grayscale Version of First Mesa Survey (Set 1)...............................66

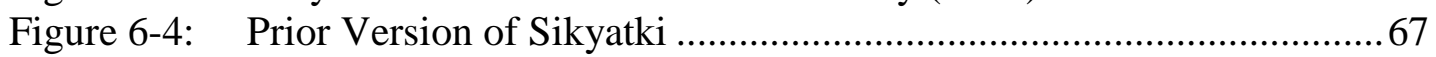

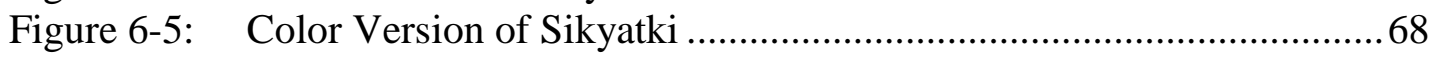

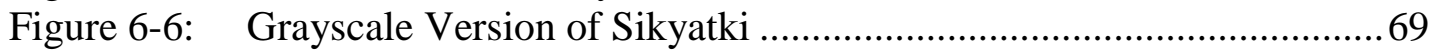

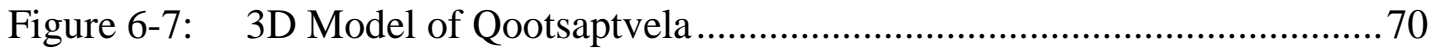

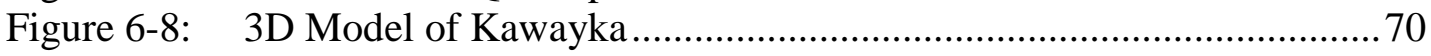


Figure 6-9: $\quad$ Survey Responses to Original and Custom Symbology Sets ........... 72

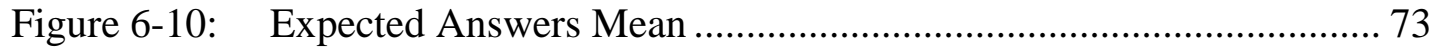

Figure 6-11: Scatterplot of Expected Answers Results ...................................... 74 


\section{List of Tables}

Table 1. Requirements List. ............................................................................ 22

Table 2. Image Strips Requested from Digital Globe Foundation............................ 35

Table 3. Features Required in Custom Symbology Set. ......................................... 42 



\section{List of Acronyms and Definitions}

$\begin{array}{ll}\text { CITI } & \text { Collaborative Institutional Training Initiative } \\ \text { DEM } & \text { Digital elevation model } \\ \text { EMF } & \text { Enhanced Metafile } \\ \text { FGDC } & \text { Federal Geographic Data Committee } \\ \text { GIS } & \text { Geographic information system } \\ \text { GPS } & \text { Global Positioning System } \\ \text { IRB } & \text { Institutional Review Board } \\ \text { NAD } & \text { North American Datum } \\ \text { PDF } & \text { Portable document format } \\ \text { SQL } & \text { Structured Query Language } \\ \text { SVG } & \text { Scalable Vector Graphics } \\ \text { TIFF } & \text { Tagged Image File Format } \\ \text { TIN } & \text { Triangular irregular network } \\ \text { USGS } & \text { United States Geological Survey } \\ \text { UTM } & \text { Universal Transverse Mercator }\end{array}$





\section{Chapter 1 - Introduction}

This project improved archaeological research on the Hopi reservation in northeast Arizona by establishing a cartographic standard for depicting excavation sites in both two-dimensional (2D) print maps and three-dimensional (3D) models. This chapter provides an overview of the project planning, design, development, and implementation phases to address the spatial problem brought by the client, Dr. Wesley Bernardini. The project complied fieldwork data over the last ten years into a geodatabase and produced a map series using a custom symbology set and 3D models of major Hopi archaeology sites to improve visualization of the fieldwork data.

\subsection{Client}

A professor in the Anthropology and Sociology Department at the University of Redlands, Dr. Wesley Bernardini's academic interests include researching the Hopi nation. For ten years, Dr. Bernardini collected fieldwork spatial data and visualized the data through a geographic information system (GIS). He used these data to illustrate excavation sites and share fieldwork observations with other archaeologists and members of the Hopi reservation, but he needed a consistent way of representing his research with a map series rather than individual maps. Dr. Bernardini provided spatial vector data representing archaeological features and raster data for this project. He also helped establish the cartographic requirements for visualizing the sites, provided input about the essential elements of each map, and evaluated the prototype symbology and map designs.

\subsection{Problem Statement}

Dr. Bernardini needed a custom symbology set to represent artifacts observed on the Hopi reservation to improve how readers interpret these archaeological data. Previously, Bernardini used generic symbology to create standalone maps and 3D models of the archaeological sites. As a result, the visual inconsistency of the original maps limited the way audiences read relationships between various sites. Prior to this project, Dr. Bernardini produced a set of custom symbols, but many of the symbols render poorly on the screen. He needed a product that represented a series of archeological sites to use in potential future publications.

Moreover, Dr. Bernardini provided spatial data located in a variety of files, causing inconsistency between datasets and making it difficult for navigation. He required a more efficient database that cataloged and maintained the integrity of these data. In particular, Dr. Bernardini required that the spatial data be in a standard projection to provide a basis for improving the cartographic functionality and overall veracity of the data.

\subsection{Proposed Solution}

With emphasis placed on organizing the data in a more systemized location and providing a cartographic standard for map designs, this project established a database that compiled the existing data and represented Dr. Bernardini's research through maps using GIS. The GIS solution included the following components: a geodatabase containing all 
the data pertaining to the project in a consistent projection; a customized symbology set representing multiple archaeological features; the creation of a map series adhering to cartographic requirements established by the client; and rendering 3D models of the inferred structures found on major archaeological sites. To determine the intuitiveness of the symbology set, a survey asked participants from the University of Redlands to interpret the symbols. The survey results provided a quantitative means to test the custom symbol set by analyzing the amount of expected answers chosen for each question.

Error! Reference source not found. shows the 48 archaeological sites within or surrounding the Hopi Reservation. The majority of sites lie along First Mesa, Second Mesa, Third Mesa, and Antelope Mesa while the outliers reside outside the legal municipal boundary of the reservation.

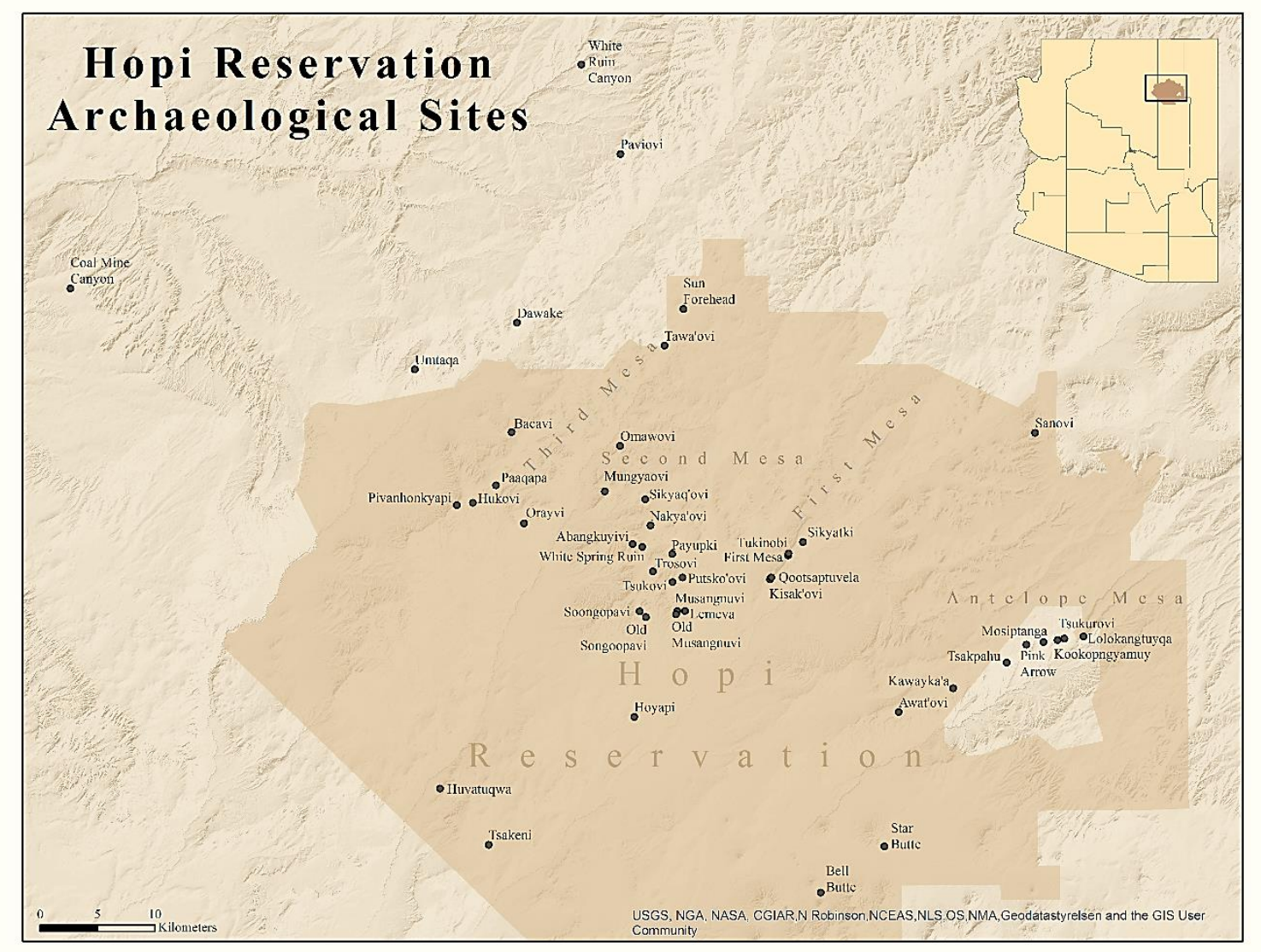

Figure 1-1: Archaeology Sites on the Hopi Reservation.

\subsubsection{Goals and Objectives}

The first objective was to design a custom symbol set representing common archaeology artifacts found on the Hopi Reservation, such as petroglyphs, stone walls, and middens that the client can access after the project's completion. A map series in color and grayscale using the custom symbol set and the 3D models of the major archaeological sites enhanced the visual depiction of the archaeological sites. Lastly, a geodatabase 
unified the input data into the same projection system to help maintain the integrity and cartographic functionality of the data.

\subsubsection{Scope}

The project consisted of four major deliverables developed using Esri's ArcGIS 10.3 and ArcGIS Pro 1.2 software. The first deliverable comprised a custom archeological symbol set that encompassed 21 major archaeological objects, such as isolated occurrences and collection units, found in the Hopi reservation and visited regularly by the client; the feature classes representing these objects included point, line, and polygon vectors. The second deliverable consisted of a geodatabase containing 595 input data files used for representing the sites. Developing a perfectly organized geodatabase became out of scope during the planning stage due to multiple data inconsistencies, but advancements during the data scrubbing process enhanced the integrity of the input data. The intent of the geodatabase provided a solution to standardize the projection system to all of the data before beginning the cartography portion of the project since the projections of the input data were inconsistent. Using the custom symbology, the third deliverable entailed a color and grayscale map series depicting each of the archaeological sites for potential publication in the client's future research. The last deliverable involved rendering 3D models of ten major Hopi archaeological sites using ArcGIS Pro 1.2. The Digital Globe Foundation provided the imagery used for the terrain overlays in the models.

\subsubsection{Methods}

Dr. Bernardini compiled and provided data in an ArcGIS compatible format as shapefiles, so this project continued to use ArcGIS since the client previously installed the software on his desktop. The ArcGIS 10.3 platform provided a means to design the geodatabase and map series; the 3D models used ArcGIS Pro 1.2; the custom point vector symbols were designed in an image and design-processing software called Inkscape 0.91 ; the Symbol Property Editor interface in ArcMap 10.3 provided a method for designing the lines and polygon fills.

The client provided input data collected through Global Positioning System (GPS) units spanning a ten-year period during fieldwork. The Digital Globe Foundation provided high definition $50 \mathrm{~cm}$ resolution imagery taken by the WorldView 2 satellite of the four mesas on the Hopi Reservation. The area of interest included all but one of the archaeological sites and surrounding geography. Imagery assisted in verifying the positional accuracy of the input data after standardizing the projection system.

Inkscape 0.91 provided multiple editor and drawing tools for designing point symbols and offered several export formats including Scalable Vector Graphics and Enhanced Metafile. The Symbol Property Editor in ArcMap 10.3 created and saved the semi-custom symbology within the software. With the symbology finalized, multiple map prototypes underwent revision until a final design received approval for the entire map series. An anonymous online survey was administered to students at the University of Redlands to determine the intuitiveness of the custom symbols and verify their functionality in the map series. The survey required approval from the University's Institutional Review Board and human subject training through the Collaborative Institutional Training Initiative. Saving the symbology in ArcMap 10.3 Style Manager 
made them readily accessible in the Symbol Selector interface. In ArcGIS Pro 1.2, the 3D models used semi-custom symbology and Digital Globe Foundation imagery. Figure 1-2 illustrates the workflow diagram for designing the products.

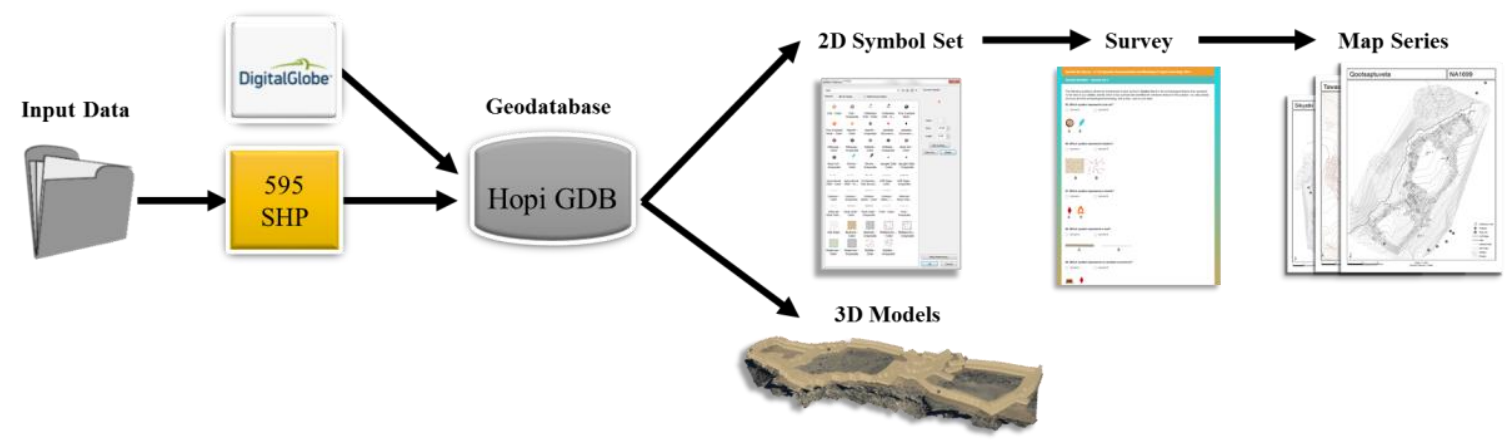

Figure 1-2: Workflow of Product Design.

\subsection{Audience}

The intended audiences for this report are those interested in applying GIS within the field of archaeology and those interested in cartography using GIS. Historians or archaeologists seeking a way to create a customized display of spatial information for their particular sector rather than relying solely on default symbology will also find this report beneficial. Although this report assumes some familiarity of GIS, a considerable amount of explanation for defining the technical and methodical terminology and concepts are included. Therefore, potential GIS users with archaeological knowledge may find the report constructive for applying spatial technologies within the discipline.

\subsection{Overview of the Rest of this Report}

The remainder of this report delves into further detail about the design, development, and implementation phases throughout the project. Chapter 2 reviews previous research regarding basic cartographic practices and the benefits, challenges, and limitations of creating custom archaeological symbols and maps using GIS. Chapter 3 discusses the overall project plan and individual tasks needed to complete the project, as well as detailed information about the system design and functional requirements of the final products. Chapter 4 addresses the information regarding the conceptual and logical models of the geodatabase design and data sourcing. Chapter 5 discusses the technical methods to develop each deliverable during the implementation phase. Chapter 6 evaluates the results of the final products and the survey data acquired after testing the symbology designs. Chapter 7 concludes by highlighting the major project elements, discussing recommendations for additional efforts, as well as notes the necessary data for pursuing these future works. 


\section{Chapter 2 - Background and Literature Review}

Spatial technologies have profoundly influenced the field of archeology since many archeologists seek to understand past human relationships with the surrounding environment. According to Harris (2002), since the birth of archaeology in the nineteenth century, archaeologists rendered sketches or watercolor maps to visualize excavation sites and the surrounding geographies. Today, the use of digital spatial technologies like GPS and GIS allows archaeologists to interactively visualize and explore sites in 2D and 3D settings throughout the excavation process. Before developing a GIS solution, examining previous works helped determine the best methodology for approaching the project. Section 2.1 reviews general cartographic principles that influenced the final design choices for the map series. Section 2.2 discusses how current applications of cartography in field of archaeology and the limitations archaeologists confront using GIS. Section 2.3 covers how archaeologists utilize 3D modeling render interpretations of building sites, and its ability to make the excavation process more efficient.

\subsection{Overview of Cartographic Principles}

Before looking explicitly at archaeology map designs, basic cartographic conventions and principles for effective map design outlined the fundamentals. Throughout the decades, cartographers have continuously analyzed and reconsidered individual cartographic elements, so acknowledging an array of cartographic theories proved excessive. Thus, this project evaluated a generic overview of the cartographic conventions before advancing to the design and development stages. Cartography must produce simple maps that clearly represent even the most complex data and not confuse the reader; otherwise, the reader becomes frustrated at what he or she does not understand or misinterpret the presented information.

Cynthia Brewer (2005), author of Designing Better Maps, noted that the most subtle of details within a map have a major impact on map interpretation. Cartographers must link visual and attribute characteristics when creating symbols. Brewer argues that "there are few excuses for using a default symbol that does not match well with the data it represents" (p.143) because cartographers have the ability to design any map symbol they envision. Due to their basic shapes, default symbols represent information generically to portray various objects. Custom symbology, or symbology that better represents a feature, is often more intuitive for the reader and therefore easier for the reader to interpret.

In The Visual Display of Quantitative Information, Edward Tufte (1983) warned against creating visual puzzles for the reader. For instance, a map with a color four-byfour grid sparks immediate visual interest, but depictions like this become enigmas for readers. Illustrating complex data can become what Tufte defines as "crypto- graphical mysteries" (p.153) that the reader has to memorize to decode, such as remembering what each color on a four-by four grid indicates when looking at the associated map. Similarly, representing data using default symbols is often difficult to interpret, leaving the reader to glance frequently at the legend to remember what symbol represents what feature.

Oftentimes, designers test if a graph or map is overly complicated if the information must 
be understood verbally rather than visually. Tufte wrote that "graphical elegance is often found in simplicity of design and complexity of data" (p. 177).

\subsubsection{Point Symbology}

Unfortunately, default symbology containing basic geometric shapes is common in many GIS products. Because of these readily available symbols, users often select a gray circle or green square over symbols that more accurately portray the features they represent. Though functional, default symbology is often ambiguous for a reader unfamiliar with the presented information since the symbols are not intuitive enough to interpret without occasionally glancing back at the map legend. Cartographers may opt for custom symbology, such as pictographs or marker symbols, to improve map legibility.

Brewer (2005) defined pictograms as icons or glyphs that use shape in a more embellished way to represent information. Generally distinguishable due to their design characteristics, a map designer should not assume universally discernibility with a pictogram symbol. Arthur Robinson (1995), author of Elements of Cartography, acknowledges the limitations of pictograms, which he calls mimetic symbols. These work when representing unambiguous data that readers understand easily, but these types of symbols becomes so intricate that reader struggle between differentiations. To prevent overly detailed pictograms, symbol set use various shapes and colors to be discernable at multiple map scales. Robinson also noted that some conventional symbology is so universal that shifting from the standard can also be problematic. For example, a cliff edge represented as a line with offset triangles showing the orientation of the ledge is a common representation, diverging from that symbology adds unnecessary confusion.

Overall, symbols must be uniquely identifiable and visually different from one another. Thus, cartographers assess the functionality of a symbol set collectively. Attributes such as color and shape need to be different enough to be visually unique, but all of the symbols must be cohesive and retain relational patterns such as size within the symbol set for a cohesive appearance (Robinson, 1995).

\subsubsection{Line Symbology}

Lines represent movement and connections between data, such as roads, rivers, boundaries, and contours. Lines illustrating qualitative data use shape and color to define different attributes, while lines depicting quantitative data may also use size. Robinson (1995) noted that cartographers must consider size before using a line symbol so readers do not interpret qualitative data as ordinal or quantitative information. According to Brewer (2005), the use of dashed lines with varying lengths represent complex patterns or arrangements. However, Robinson (1995) cautioned, "such symbols may be considered point symbols rather than line symbols. The distinction between whether a symbol is a point or line symbol becomes rather subjective" (p.484). Therefore, cartographers design dashed line segments with minimal spacing, so data do not appear as point symbols systematically aligned on a map.

This project worked with contour lines derived from individual control points noting the elevation using a GPS unit. Intervals of the input contour lines varied between sites, ranging from 3 meters, 2 meters, 1 meter, 0.5 meters, 0.25 meters, 0.2 meters, and 0.1 meters, which made it difficult to determine one method for representing contour 
lines. According to Edward Imhof (2007), contour lines depict the metric location of point data with the same elevation to represent topography and assumed to retain consistent numerical distance between each other called contour intervals. Imhof claimed that the smallest contour interval possible be selected depending on the scale in order to provide more detail about the topography of an area; however, he warned that using small contour intervals leads to overcrowding and are challenging to read. To make for easier legibility, contours need bolder index contours that represent simple, whole numbers; map scale determines the index contours. Generalization simplifies Earth's complex curvatures, especially when working with smaller map scales. Larger scaled maps at 1:100,000 or less need to retain more detail (Imhof, 2007).

Variation of line size and color make for easier visualization and interpretation. Imhof (2007) discussed how cartographers use color to represent variation in the topography to illustrate change in groundcover, elevation, or illumination. To indicate groundcover, Imhof used the following three-color conventions black for gravel or stone, blue for glacier surfaces, and brown for earth or vegetation groundcover. A color ramp also shows elevation changes, but Imhof stated, "This is a waste of time, since contours often occur on maps at widely spaced intervals... Also, it would be technically too complicated and expensive" (p.143). Cartographers need to consider line weights to maintain balance between index contours and intermediate contours. Heavy index contours result in the topography appearing as a series of steps rather than intervals. In contrast, thin index contours reduce the functionality of a map since intermediate contours appear the same weight (Imhof, 2007).

\subsubsection{Polygon Symbology}

Polygon pattern fills are literal or abstract in design to illustrate complex relationships between areas. Fills with repeating stone patterns represent rocky terrain in a literal manner while larger cross-hash fills appear as abstract. If spaces between the features in the polygon area are fine or sparse, this signifies lower values, while coarser or clustered patterns represent higher values (Brewer, 2005).

However, some researchers criticize the use of pattern fills when displaying quantitative data. As Edward Tufte (1983) wrote, "moiré vibration is an undisciplined ambiguity, with an illusive, eye-straining quality that contaminates the entire graphic. It has no place in graphical design" (p.112). Tufte explained that moiré vibrations or optical illusion patterns hinder the reader's ability to interpret the data; this is true for both graphs and map designs. He defines optical patterns and excessive ink used only for an artistic effect as "chartjunk" (p.107). Tufte argues that the use of patterns increased drastically since computers but that having the ability to produce artistic graphs does not mean that these designs represent data well. Tufte explained this concept using the infamous Big Duck duck-shaped store, discussing that though architects possess the tools to produce such a uniquely shape building, it distracts from the main purpose of the building. In other words, simplicity communicates information clearly, whether though a storefront or a map. Decorative patterns quickly clutter or confuse the simplest of information. Tuft argued that abandoning excessive decoration or needless ornamentation is an ornate design decision within itself (Tufte, 1983).

Though heeding Tufte's (1983) caution of moiré patterns, the final map designs included pattern fills to help readers visualize the archaeological sites. Rubble, for 
example, is an archaeological feature of scattered stones that infer that some structure previously existed. The use of a solid color fill in this instance would hinder the reader's ability to envision the site. The uses of brown circles of varying sizes randomly scattered in the area represent rubble more efficiently than a solid fill, yet the randomization of the repeated symbols prevents any optical illusion.

\subsubsection{Working with Color}

Many cartographers consider color one of the most captivating aspects of cartography due to their subjectivity and no set color rules. Arthur Robinson (2010) wrote, "of all the media of cartography, color is probably the most complicated and least understood" ( $p$. 75). Many cartographers study color and to research every theory on color would prove too demanding for this project, but the following section discusses key components to common color theory practices.

Some conventions, such as the United States Geological Survey (USGS) topographic maps, use standards agreed upon to keep maps consistent within an organization. Others look to common logic for color choices. In The Look of Maps, Robinson (2010) noted these cartographic conventions:

- Blue hues for hydrology

- Warm colors for rising or heat; cool colors for decreasing or cold

- Yellows and tans for dry areas with little vegetation

- Greens for vegetation and lush environments

- Browns for ground covers and contour lines

Robinson (2010) argued that color is difficult to describe, which results in a limited understanding of how colors function and express sensations. The eye notes colors though physical variations of hue, value, and intensity. However, the surrounding environment affects the visual characteristic of color even though the physical traits remain the same, known as simultaneous contrast (Robinson, 2010). Figure 2-1 provides an example of this phenomenon where different environment hues encompass the same gray hue in the center square. Some color combinations are more effective than others are.
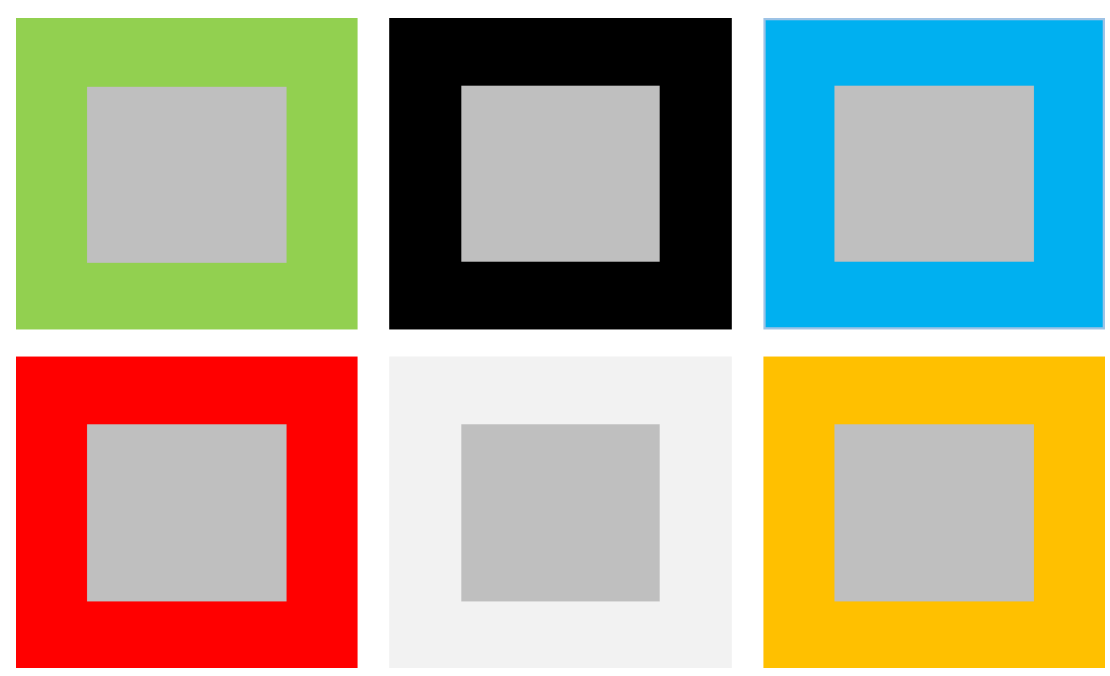

Figure 2-1: Simultaneous Color. 
In Principles of Thematic Map Design, Dent (1985) claimed that most cartographers opt for color maps given the increase in design creativity. The use of colors produces three different interactions according to Dent: simultaneous color, successive contrast, and color consistency. Simultaneous contrast is produced when colors that are opposite on the color wheel, or complementary colors, are used together on the same map. Surrounding one complementary color next to another color produces simultaneous contrast, and eventually, the two colors begin to take the same hue of the surrounding color to create the illusion that it is the same shade but with different illumination. Lastly, the perception of illumination from one common light source generates color consistency. This creates the effect of synthetic shadows depending on whether the color contains gray or not (Dent, 1985).

Dent (1985) also discussed that colors stimulate different psychological responses in individuals, which is why researches considered color subjective. However, some known color combinations make for more or less effective maps as discussed in Dent's research, which tested 10 different hues against 25 random background colors. The results were:

- Appealing color combinations ranged in different lightness values.

- Light or dark basemaps are the most effective.

- In general, colors with green, blue, or gray are considered the most visually appealing.

- The least appealing colors are yellow and yellow-green.

- Object colors should stand out against the background.

- Effective and non-effective color combinations are found anywhere on the color wheel.

Dent (1985) also considered three of Robinson's color theories for cartography. First, color is a simplifying method by developing figure and ground. Second, color affects the legibility of the map by making the most important information more distinct. Lastly, people respond differently to the same hues. Figure and ground development are essential for producing simple and legible maps. Objects of interest, or figure, stand out against the basemap containing reference information called ground. Retreating colors help promote figure and ground to create a visual hierarchy. High value warm colors such as yellow, orange, and red often represent crucial information, while cool colors such as greens and blues are visually less prominent and retreat into the ground. As colors reduce in saturation, the colors also recede to the back of the map (Dent, 1985).

\subsubsection{Text and Labeling}

Typefaces set the tone for a map, but text used on a map needs to be easily legible regardless of the size or orientation. From traditional to contemporary, serious to animated, official to whimsical, typefaces evoke specific tones towards information presented in maps. Brewer (2005) classified typefaces into three different categories. The following provides examples of the classifications:

- Serif: Times New Roman, Garamond, Century

- Sans Serif: Calibri, Arial, Verdana

- Display: Broadway, Papyrus, Monotype Corsiva 
Ornate strokes found on the ends of each letter signify serifs; sans serifs remove the additional strokes; and decorative display fonts often contain exaggerated characters. Maps commonly use serifs or sans serifs and generally avoid display typefaces due to their overt appearances with only some exceptions for titles and logos (Brewer, 2005).

Brewer (2005) claimed that the relationship between features influence the font, color, and symbol size to determine the reading order of the features. In general, larger fonts demand attention while smaller fonts subdue into the background. However, carefully considered color and widely spaced characters fade larger fonts into the background to span across a geographic area and make smaller labels increase on the visual hierarchy scale. Topographic features use sans serif fonts, while other geographic features such as cities or boundaries use serifs. Labels are a blue hue for hydrographic features, while labels for contour lines are usually the same color as the contours. These labels should curve around these linear features to increase legibility, while straight labels denote municipal features. Ideally, point features labels are placed in the top-right corner with wrapped text if necessary (Brewer, 2005). Figure 2-2 shows examples of the basic labeling conventions used to label points, lines, and polygons.

\begin{tabular}{|l|l|}
\hline $\begin{array}{l}\text { Point features are labeled in } \\
\text { the following order for } \\
\text { effectiveness. 1 preferred; } 6 \\
\text { least preferred }\end{array}$ & $\begin{array}{l}\text { Hydrology features are } \\
\text { often labeled in blue italics } \\
\text { and curve with the natural } \\
\text { feature. }\end{array}$ \\
\hline 2 & $\begin{array}{l}\text { Labels should reside within } \\
\text { the polygon, but if the } \\
\text { polygon is too small, text } \\
\text { should be next to the label } \\
\text { and possibly wrapped. }\end{array}$ \\
\hline Contour lines are often \\
brown with index intervals \\
labeled on the line center in same color.
\end{tabular}

Figure 2-2: Labeling Hierarchy and Conventions. 


\subsubsection{Map Layout}

Clean marginalia is key in designing a map, so the layout of the map series mitigated any visual awkwardness by selecting a simple, official layout design over a unique, artistic one since the information may be published at smaller scales in articles. In addition, the client can easily replicate the minimalistic design for future maps without spending extraneous time on the map layouts. Brewer (2005) noted that experimenting with map design elements can open new avenues for more effective map displays and that novice cartographers often fill every space using large text or ornate elements.

Brewer (2005) also warns against over-boxing elements since a box possesses a distinctive shape that blocks certain spaces and can be difficult to incorporate in the layout. Text and other map elements within a box can create distracting shapes or overcrowding. Brewer wrote that by removing the box, elements are more organic in design and form open spaces that are easier to balance with other marginalia, such as an inlay map or legend. In addition, map elements that possess a conceptual relationship worked best when physically located in close proximity to one another. Once these relationships are determined, elements on the map must be aligned or overtly unaligned to establish a firm design decision; slightly skewed elements appear as mistakes. Failure to consider these relationships makes for an unsophisticated and unpolished map that is confusing to interpret.

The maps designed for the Hopi archaeological sites use an upper-left to bottomright reading schema with the most important information starting at the top and working down to the lower-right corner of the map whenever applicable. By removing the lines that formed boxes, elements appeared less cluttered and did not generate unnecessary shapes; only a few exceptions existed. Boxing the names of sites and catalog numbers invoked a more official layout. Though removing the border on the inlay map was preferred, it became problematic to maintain differentiation between the maps and inlay since no basemap existed to provide a more organic transition. Removing the default boxes around the legend make it less distracting from the actual map.

\subsection{Cartographic Designs in Archaeology}

Archaeology is a spatial discipline. Anne Knowles (2005), a specialist in historical GIS, acknowledged that archaeology was the first of the humanities disciplines to implement spatial technologies. Since archaeologists understand fieldwork and study human relationships to geography, many archaeologists transitioned towards digital spatial methods. Today GIS is widely discussed in many archaeology textbooks and commonly used to analyze aerial photography, imagery, and GPS data collected during fieldwork (Knowles, 2005). Utilizing spatial technologies helps researchers visualize excavation sites to analyze spatial patterns and relationships between locations and individual features. They also aid in locating artifacts and predicting future excavation work more accurately. However, unlike many other spatial disciplines, archaeology often works with ambiguous artifacts from various time periods sometimes located in the sub-terrain. Representing time, space, and uncertainty are some of the limitations of GIS since it focuses on discrete objects. Due to these limitations, the use of 3D modeling to aid archaeological studies increased within the last decade in order to represent two of these three elements: space and time. 
Though a considerable amount of research focuses on archaeology and GIS, few have considered the visual aspect and need of cartographic standards within the discipline. Traditionally, many cartographers hand-drew with pen and ink to depict archaeological features on a map. However, the increasing use of GIS has left many cartographers concerned that intermediate analysis maps are being portrayed as final presentation maps, limiting the audience's ability to interpret the information.

Archaeology is no exception to this rising concern; presenting data in a well-designed manner is crucial for communicating archaeological fieldwork.

\subsubsection{Current Archaeological Symbol Sets}

After reviewing an archived forum from the Esri Discussion website in 2005, many archaeologists questioned whether an archeological symbol set exists in the ArcGIS platform. In the discussion, Bob Booth (2005), the Archaeology Group Coordinator at Esri, stated that the company did not have an established symbol set for archaeology, but that Esri does have a set of fonts, Cave 1, Cave 2, and Cave 3, which contain many applicable icons. Looking at many of the ArcGIS symbol font selections, there are a few general markers to represent archaeological sites. However, these are only indicators of excavation areas, not of actual features found on the sites. On the other hand, the Caves typefaces consist of many relevant symbols though many of these are not suitable when filling polygon features. Midden, for example, is an area of scattered small discrete artifacts, yet the Caves fonts represents midden as a point feature. Locating the Caves fonts is also a tedious approach to symbolize maps for some archaeologists since they must have the knowledge and time to edit symbols through the ArcMap Symbol Property Editor interface.

In a presentation given on managing cultural data, Kier Keightly (n.d.) discussed the need to unify maps and produce a symbol set for fields like archaeology. The goal was to help access large historic and prehistoric sites by establishing "spatial analysis and cartographic products" (p. 2). Specifically, the Merced Irrigation District required a way to maintain the McClure and McSwam reservoirs that contain over 286 archaeological sites that consist of approximately 4,300 artifacts and other archaeological features. To represent these findings, researchers formed a unified symbol set. These symbols, according to Keightly, tried "to emulate traditional illustrated [archeological] sketch maps" (p. 6) and were a combination of USGS and archeology symbols. The set included basic diamonds for artifacts, circles for specific features, a custom symbol for rock art, and many other line and fill symbols.

The Cal Fire Archaeology Program also possesses its own custom symbology set to produce maps for the Archaeological Site Records. These records consist of three types of maps. Sketch maps are conceptual hand-drawn maps used to create the final product maps, which include a small-scale location map using the USGS topology quadrangles and a detailed large-scale archaeological site map. The use of these symbol sets is limiting, however, since its purpose was to establish a visual consistency for Cal Fire archaeological projects and were not appropriate for universal use.

There is little information on symbology sets for the field of archaeology, and even less for representing archaeology sites in the American southwest. Though Elisabeth Cutright-Smith's (2013) dissertation titled "Mapping Ancestral Hopi Archaeological Landscapes" reported on the methodology of procuring a GIS solution and the benefits of 
using GIS to visualize the Hopi environment, the maps within the report became a catalyst in understanding a need for a symbol set specifically for the Hopi nation. The symbology used to produce the maps used default symbology in vibrant colors and worked well to contrast against the imagery. However, the default symbology did not evoke the Hopi cultures and required frequent glances back at the legend to remember which symbols were associated with what feature.

Figure 2-3 summarizes the four symbol sets found to represent archaeological features. The array of symbols illustrates that each sector requires its own unique symbology to represent certain features. The symbology sets designed by Cal Fire (a) and Kier Keightly (n.d.) (b) are comprised mostly of topographical and natural features; the majority of the Esri's Caves (c) fonts are for point features and have little to no line and polygon symbology; and, the symbology used by Elisabeth Cutright-Smith (2013) (d) are solely point symbols representing man-made features.

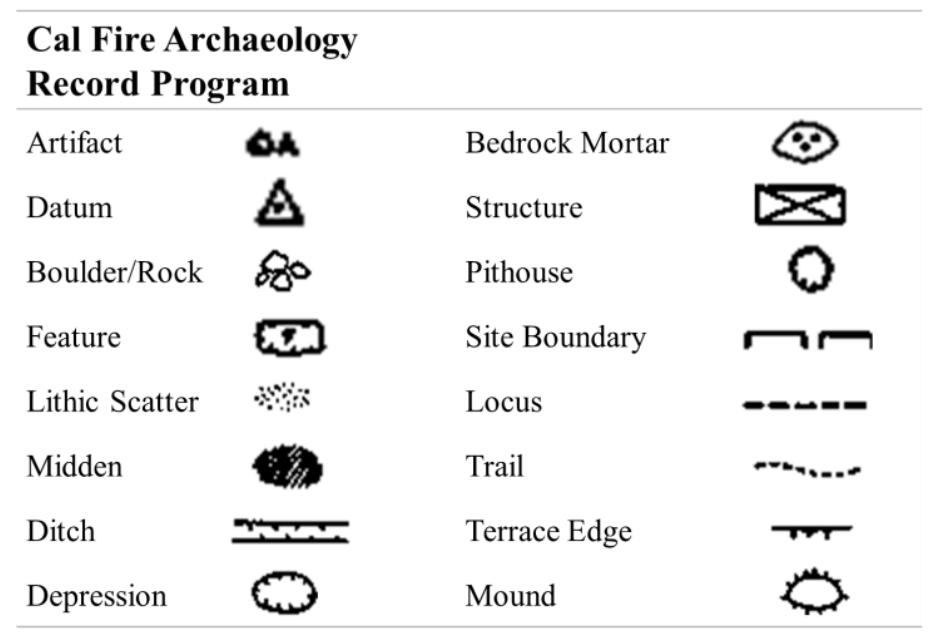

(a)

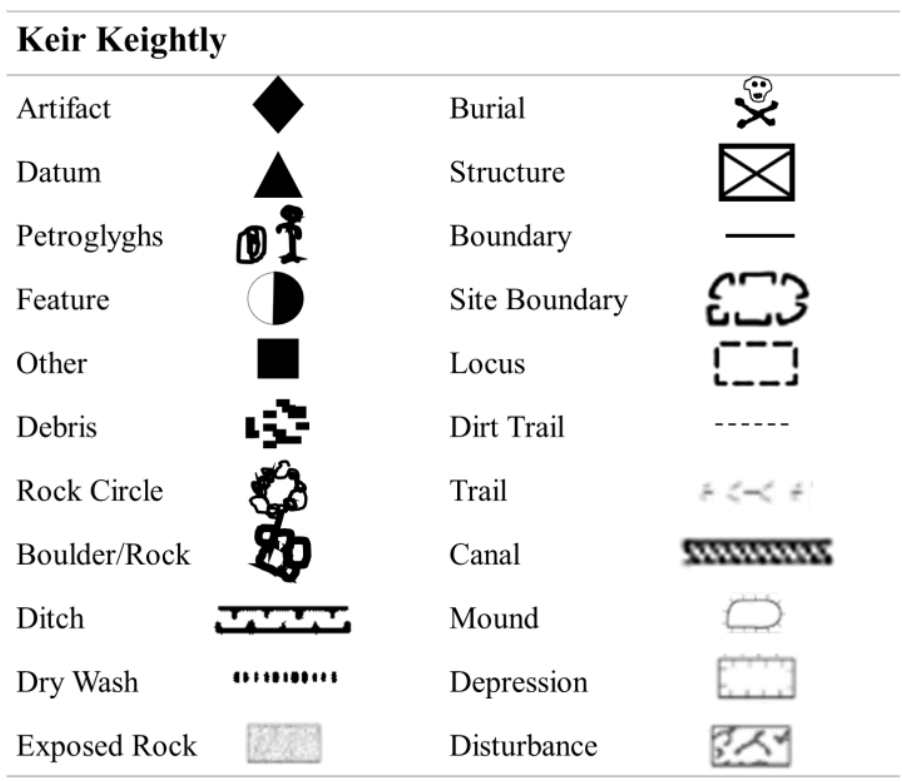

(b) 


\begin{tabular}{|c|c|c|c|}
\hline \multicolumn{4}{|l|}{ Caves Font } \\
\hline Artifact & 0 & Wall & 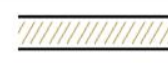 \\
\hline Datum & 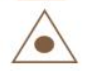 & Inferred Wall & \\
\hline Boulder/Rock & 808 & Hearth & $\mathrm{H}$ \\
\hline Feature & $\mho S$ & Mound & 89 \\
\hline Petroglyph & $r>-$ & Test Pit & $\phi$ \\
\hline Midden & $M$ & Rubble & $\underbrace{}_{5}$ \\
\hline
\end{tabular}

(c)

\begin{tabular}{ll}
\hline Elisabeth Cutright-Smith \\
\hline Artifact
\end{tabular}

(d)

Figure 2-3: Four Observed Archaeological Symbol Sets. Symbology adapted from "Cultural Resource Data Management and Mapping Using GIS: A Unified Approach for a Large Scale FERC Relicensing Project" by Keightly, K., "CALFIRE Archaeological Program" by Calfire Archaeological Record Program, by Esri Cave Font, and "Mapping Ancestral Hopi Archaeological Landscapes: An Assessment of the Efficacy of GIS Analysis for Interpreting Indigenous Cultural Landscapes" by Cutright-Smith, E.

\subsubsection{Limitations of GIS in Archaeology}

Many cartographers debate that the quality of maps significantly declined since the use of spatial technologies (Howard, 2007). Howard (2007) noted that GIS left cartographers upset by the poor use of representation and absence of cartographic principles applied when trying to communicate information in archaeology. Howard wrote, "Surveys portrayed have been deliberately straightforward, but the nature of archaeological information (or many other kinds of information recorded in the field) is seldom so simple" (p. 108). Howard argued this is partially due to a plethora of representation choices provided to the producer who often forgets that homogeneity and simplicity are key to communication. When communicating archeological information, it is vital to consider how effectively and quickly readers can interpret the information given to them, 
but this is often difficult given the need to illustrate a usually complex set of information (Howard, 2007).

Representation of archeological information varies depending on what type of feature is being represented in relation to its location and time in the real world. For instance, a symbol of a blue feather may work well depicting Native American shrines in the American southwest, but the same iconography may not be applicable to other places of worship around the world. Another challenge for archaeologists is to represent structures with the obscurity of what that structure looked like in the past. Archaeologists often work with uncertainty and theorize that a structure existed at a certain location. Traditional maps used stippling, or a hand-drawn random dot pattern, to represent uncertainty. Archaeology rarely deals with discrete object and stippling was a common technique to represent rubble and stone wall ruins. Figure 2-4 contains a photocopied image of a map using stippling to represent Payupki published by the Smithsonian Institution Bureau of Ethnology (1880, p.59). However, since GIS is designed to work with concrete objects, it is limiting in its ability to represent uncertainty.

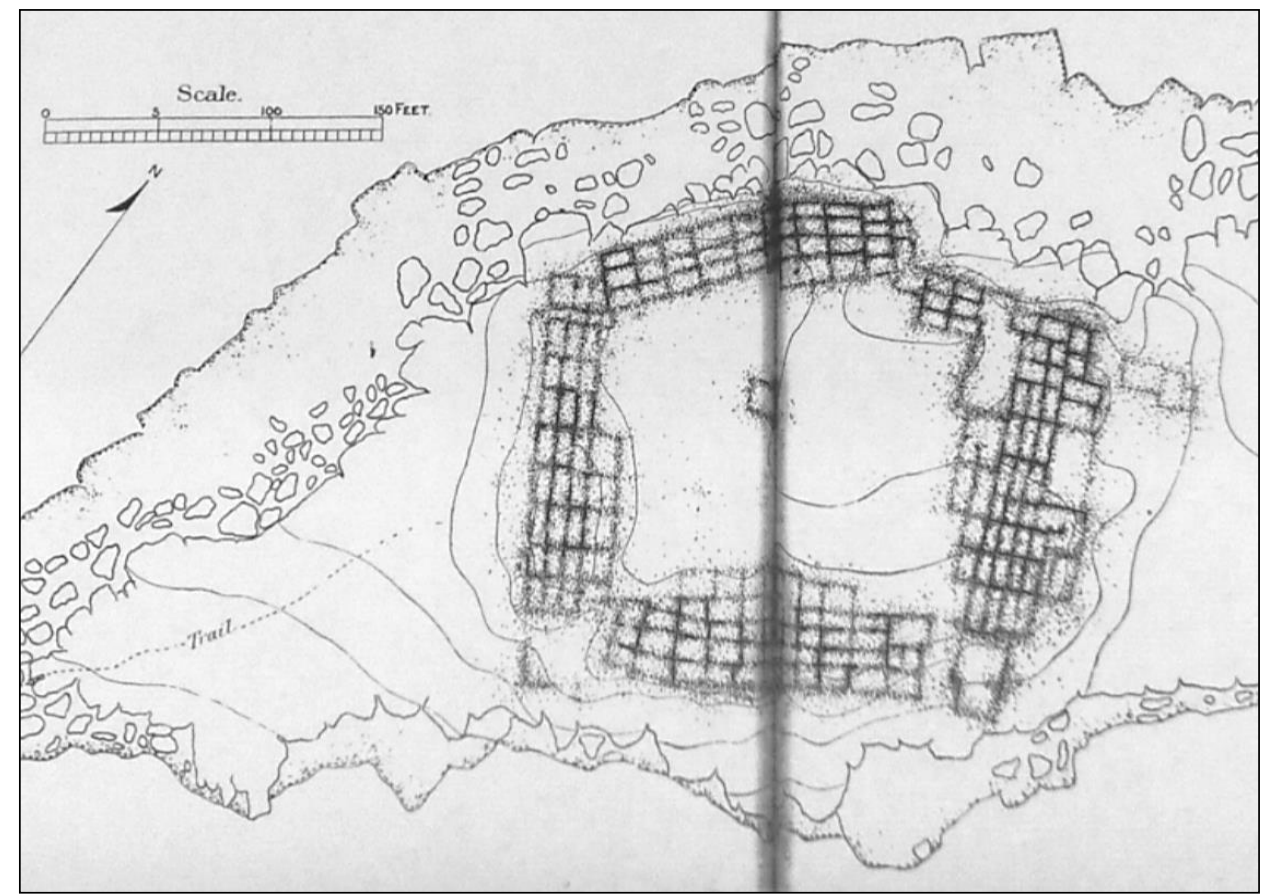

Figure 2-4: Traditional Archaeological Map. Image photocopied from Annual report of the Bureau of Ethnology to the Secretary of the Smithsonian Institution by Smithsonian Institution. Bureau of Ethnology, 1880, Washington: G.P.O., p. 59.

Howard's (2007) distinction between geographic technologies and maps is also something to consider. While geographic technologies do produce maps, Howard argued that GIS mainly functions as a database with a spatial emphasis and is not designed for cartographers. GIS may perform well at recording archaeological information, but that does not mean that spatial technology is able to represent information effectively through a cartographic lens. 
Nevertheless, some archeologists debate if database systems in geographic technologies are without fault. Kvamme (2012) discussed the problems archaeologists confront when working with spatial data such as imagery, GIS, and how to represent visualization. In summary, Kvamme (2012) defined representation as a way to depict "past places or objects by rendering them through advanced computer graphic methods" (p. 4). Using 3D modeling has allowed archaeologists to study virtual representations of the world in order to understand past cultures. However, with these advances in archaeology, Kvamme argued that the quality of data and their standards are of concern, not just the cartographic aspect. He proposed that an "archeological cyberinfrastructure" (p.338) must be established for regional, and possibly global, archaeological efforts as a way to standardize information. This may be due to a limited knowledge of database management overall and has caused the functionality and integrity of spatial site survey data to decrease. Inconsistences include attributes schemas or how data with various projections render on screen. Nevertheless, the construction of a well-organized geodatabase and a standardized cartographic system will assist in the functionality and effectiveness of GIS within the discipline.

\subsection{Modeling Archaeological Sites}

With rapid enhancements in satellite imagery and 3D GIS, archaeologists are able to visualize sites in 3D, arguably 2.5D since it draws on a computer screen, for further analysis and allowing for minimal excavation for conservation. One of Harris's (2002) studies included a 3D model of predictive Romano-British sites throughout England. The results of this project allowed archaeologists to visualize early settlements in Danbury, a well-known archaeological site with records from the Paleolithic civilizations to the Romano-British period. According to Harris, "By adding elevation data and displaying archaeological sites as three-dimensional images, we could more readily see how the location of ancient features related to topography and how the human landscaped changed over time" (p.132). Unlike static 2D maps, 3D models create virtual environments that allow archaeologists to consider time and space to predict what sites may have looked like in the past. Harris also noted that archaeologists find superimposed artifacts in the sub-terrain that range from various eras. The difficultly for using 2D maps is that these layers and time periods converge together as a single image, limiting archaeologists' understanding of the temporal relationship between features. Harris concludes that using 3D GIS helped locate the geographical position and depth of phosphate concentrations to verify site locations and determine how human behavior changed over 2,000 years in Danbury.

Rendering 3D models is also spreading to underwater excavations. In 2008, the Virtual Exploration of Underwater Sites project constructed a 3D virtual model of underwater archaeological sites from the 2006 Pianosa mission. Photogrammetric methods collected information about the topography of the sea floor. Researchers used an underwater remote vehicle to gather archaeological data on the sea floor (Drap, P., Seinturier, G., Conte, A., Caiti, D., Scaradozzi, S., Zanoli, M. \& Gambogi, P., 2008). Since underwater missions are expensive and often dangerous, using geospatial technologies to produce virtual models is of great value. However, representing underwater archaeological information is not an easy task. According to Drap, "in the context of archaeological survey, 2D representations are well known and largely used by 
archaeologists. These representations can be handmade drawings or digital maps" (p. 7). Drap argued that print map representations are quite useful since they are convenient for most archaeologists out in the field, use basic geometric shapes for representation, and use standard tools for terrestrial research. Since the ocean is a volume, however, representing an underwater archaeological site on 2D maps can only do so much; thus, the creation of 3D models and 3D symbols became essential to depict a realistic environment (Drap et. al, 2008). Nevertheless, cartographically illustrating excavated artifacts deep within the ocean floor is a massive task. Though Drap stated that mosaicked tiles were visually rudimentary, using photogrammetry and 3D graphical designed symbology created a virtual environment to visualize the underwater excavation sites in Pianosa.

\subsection{Summary}

While many archaeologists use GIS and a significant amount of research exists on the issue, the representation of archaeological information undergoes little investigated. Each culture has its differences, and symbology must represented those differences. The challenge is that archaeology sites vary temporally and geographically, so iconic symbols must change with these variables. The end use of a GIS product is to communicate information about an excavation site or other area of interest. If representation is not considered and cartographic principles are not applied, the information is often visually confusing or misinterpreted. The creation of a standardized archaeological symbol set and established cartographic principles is a promising investment for archaeology on the Hopi Reservation. 



\section{Chapter 3 - Systems Analysis and Design}

This chapter provides a detailed analysis of the system design that provided the client with a GIS solution for visualizing archaeology sites on the Hopi reservation. Section 3.1 revisits the spatial problem proposed by the client and details the final products delivered to the client upon the project's completion. Section 3.2 explains the functional and nonfunctional requirements of the system design. Section 3.3 discusses the system design structure in detail and notes how it satisfied project requirements. Lastly, Section 3.4 compares the original project plan and prior assumptions made during the planning phase to the final project plan and actual time constraints.

\subsection{Problem Statement}

Over a ten-year period, the client collected site survey data during fieldwork using a variety of GPS units. Data collection and recording methods became inconsistent due to the different projection systems and attribute schemas. The client produced simple standalone maps that used various generic default symbols representing fieldwork data. Often the same symbol selected to depict one archaeological object on one map represented another object on a different map. Figure 3-1 depicts an asterisk symbol used to portray hearths, potholes, and bedrock mortars in three different maps. Using the same symbol to represent various features on different maps limits the way readers interpret the maps collectively and requires users to refer to the legends frequently. 


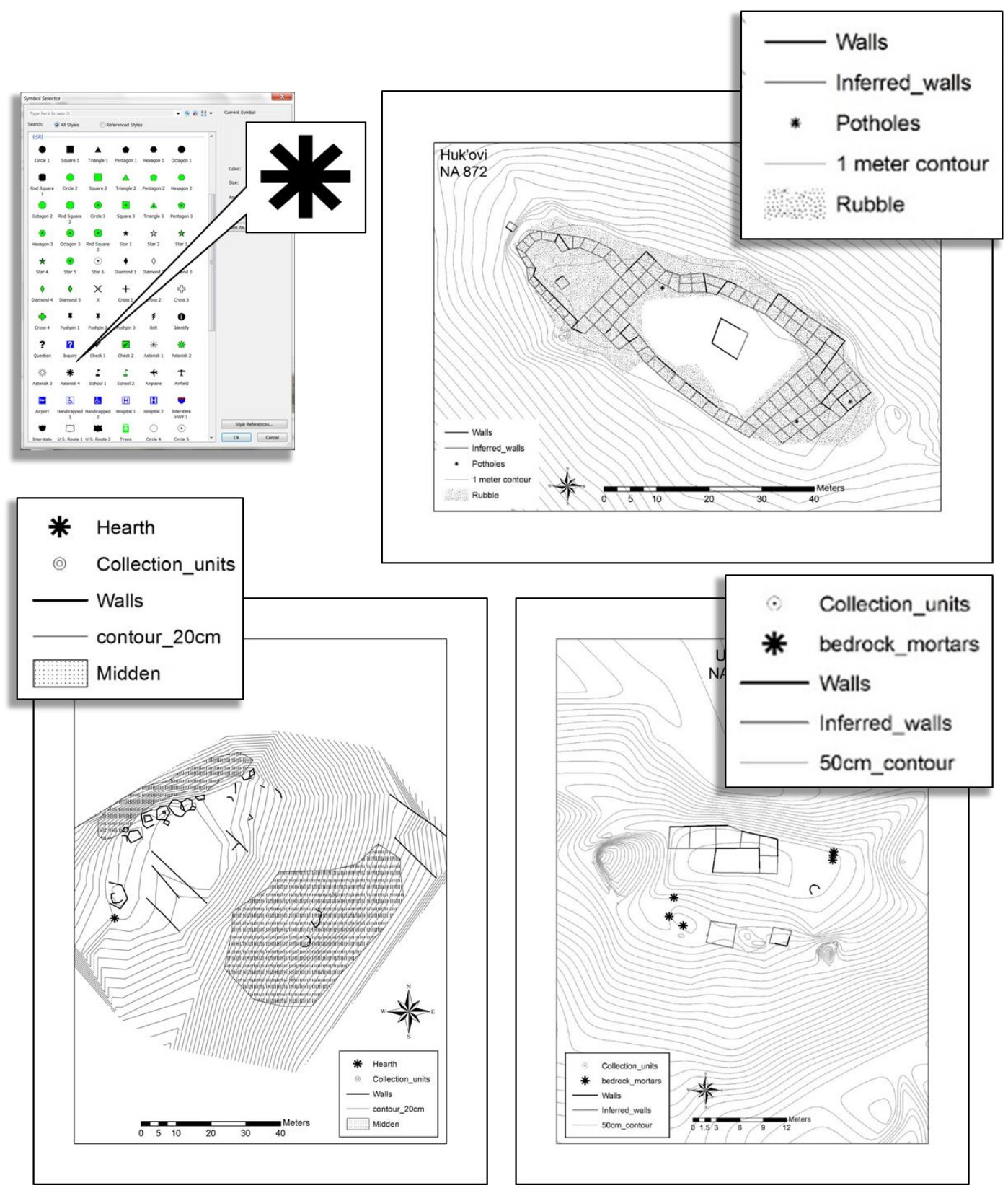

Figure 3-1: Original Map Designs Using the Asterisk Symbol Inconsistently.

\subsection{Requirements Analysis}

One of the main requirements ensured that the custom symbology set created to produce the map series satisfied the cartographic requirements defined by the client. Dr. Bernardini assembled a list of 21 commonly seen artifacts and required custom symbology that represented the Hopi people. The client also wanted the symbols created in grayscale and color for printing and be similar to traditionally styled maps. The symbology needed to be uploaded in ArcMap 10.3 and selectable in the Symbol Selector interface with the ability to change the symbol size. By conducting a survey, the 
intuitiveness of the symbols tested the symbology set by a quantitative means with undergraduate and graduate student participants at the University of Redlands.

The second requirement was a map series of the sites in grayscale and color using the symbology set. Exporting the maps in portable document format (PDF) provided Dr. Bernardini with a digital map series. In addition to the grayscale and color versions of the maps, the client also wanted a series of maps showing the archaeology sites with and without the inferred walls layer. Later research discovered that PDF formatting exports layers individually to allow the client to select or unselect visible layers within the PDF, reducing the assumed amount of maps needed for the series.

The third requirement produced 3D models of the major Hopi ruins using a semicustom 3D symbol set and imagery provided by the Digital Globe Foundation. The 3D symbol set used symbols similar to the 2D versions in the same colors but used shapes that are more geometric at a specified height value. These 3D models used the client's original 3D data for the building structures and overlaid landscape imagery to generate a more realistic view of the study areas.

Another requirement was to transfer the data into a geodatabase since it offered a more efficient format for easier navigation by categorizing data though each archaeological site with individual data layers associated with the site. The client also required standardized data in Universal Transverse Mercator projection using the 1983 North American Datum (NAD83 UTM Zone 12N). Archaeologists commonly use the UTM projection, and Arizona resides in Zone 12. The client wanted the data in the most recent datum since the majority of input data used NAD27. While not a requirement, the client suggested to begin data normalization efforts if time permitted. Due to post processing on some of the data over a ten-year period, some data were exposed to different projection tools. As a result, some data positioned inaccurately caused discrepancies with the cartographic aspect of the project because layers overlay inaccurately. Since the client retained better knowledge of the locations of the artifacts and the basemap imagery resolution made minute objects indiscernible, the client agreed to help manually shift the inaccurate data to their appropriate locations after being batch projected.

There were three non-functional requirements for this project. The first requirement stated that all the project data needed metadata that adhered to Federal Geographic Data Committee (FGDC) metadata standards. The second requirement tested the intuitiveness of the custom symbols after receiving validation of the designs from the client. The client then reviewed map design prototypes until agreeing upon a version for the final map series. The following table summarizes the functional and nonfunctional requirements: 
Table 1. Requirements List.

\begin{tabular}{|l|l|}
\hline \multicolumn{1}{|c|}{ Functional Requirement } & \multicolumn{1}{c|}{ Description } \\
\hline $\begin{array}{l}\text { ArcMap 10.3 File } \\
\text { Geodatabase with } \\
\text { standardized projection. }\end{array}$ & $\begin{array}{l}\text { File Geodatabase housing feature datasets of the } \\
\text { archaeological sites with associating feature classes. } \\
\text { All feature classes with a uniform projection. }\end{array}$ \\
\hline Symbol set interface. & $\begin{array}{l}\text { Custom symbols are readily available and searchable } \\
\text { by tags in the Symbology interface in ArcMap 10.3 }\end{array}$ \\
\hline Digital map series & $\begin{array}{l}\text { The } 48 \text { archaeological sites are mapped both grayscale } \\
\text { and color and saved as PDFs with exported layers }\end{array}$ \\
\hline ArcGIS Pro 1.2 3D Models & 3D models of the major sites with DGF imagery \\
\hline $\begin{array}{l}\text { Data Normalization and } \\
\text { Consistent Attribute Schema }\end{array}$ & $\begin{array}{l}\text { Though not a requirement, efforts were made to } \\
\text { normalize the data by writing a Python script to add } \\
\text { and populate three attribute fields for all data }\end{array}$ \\
\hline Non-Functional Requirements & $\begin{array}{l}\text { Point symbols designed for 10 point or higher but } \\
\text { discernible at smaller points. A survey tested the } \\
\text { intuitiveness of symbols with graduate and } \\
\text { undergraduate participants. }\end{array}$ \\
\hline Metadata & $\begin{array}{l}\text { Used Data Reviewer to test that all metadata adhere to } \\
\text { FGDC standards. }\end{array}$ \\
\hline The client visually critiqued map and symbol designs \\
\hline
\end{tabular}

\subsection{System Design}

The client shared the input data via the University of Redlands Spatial Studies server, which allowed the client to access the working deliverables from his desktop during the project's development. The database component of this project housed all of the spatial data used for designing the map series and 3D models. The database is accessible through the university's shared server in the client's personal folder. The client also has access to the database from his personal desktop. The custom symbology set allows the client to select a symbol on the Symbol Selector tab in ArcMap10.3. The symbol set is saved in a style format and loadable using the Symbol Manager. Tags for each symbol make it suitable for finding the custom symbols within the Symbols Selector user interface in the 
search window. If the client wants to share the symbols, he is able to send the style file to the end user, who can add it to the Style Manager on a desktop with ArcGIS installed. The map series components are saved as PDF files and stored on the server. The PDF format allows layers to be exported individually, so features can be turned on or off within the PDF to give the client more control of what to share with the public. The ArcMap 10.3 and ArcGIS Pro 1.2 files were also saved in a folder, so the client can download the files or open them within the server. Figure 3-2 shows the system design for the project.

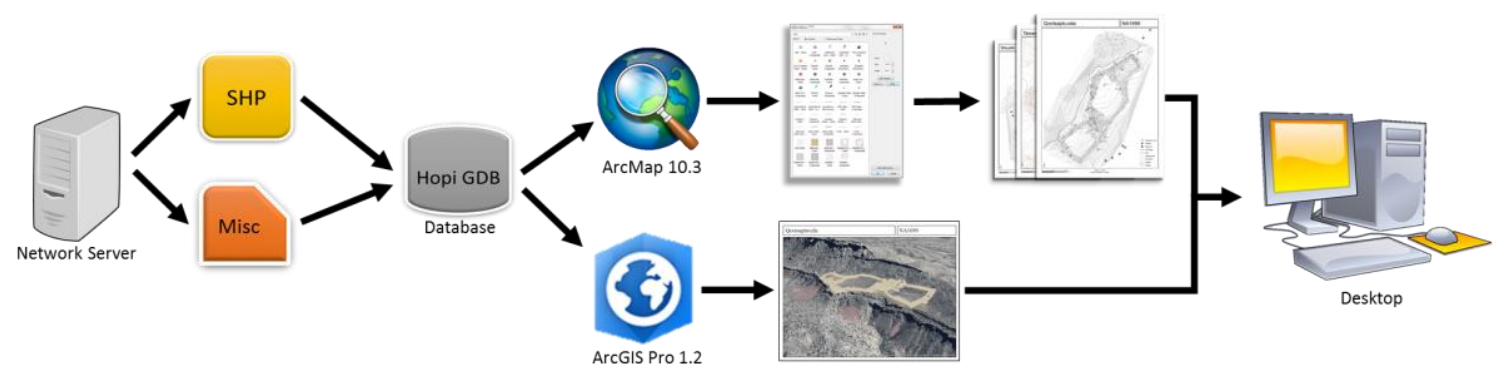

Figure 3-2: System Design.

\subsection{Project Plan}

Assuming that the input data would be acquired in November, the majority of data was actually received in December and took 12 hours to download from the server. The project began in December 2015 with a scheduled completion date in July 2016. The period for completion was accurate, but underestimating the expected hours of labor required additional hours to finish each task. As the project progressed, it became apparent that the initial time assigned for compiling the data, designing the symbology set, and producing the map series did not satisfy the actual time needed to complete those milestones. Though attempting to adhere to the original project plan, the periods for completing each task evolved throughout the project. Rather than being a static document as first assumed, the project plan actively changed as new tasks emerged and methods for completing tasks changed.

In the original project plan, March was the anticipated completion month for data scrubbing and designing the geodatabase. A significant amount of time went to data scrubbing, reconnecting some data pathways, projecting the data, and verifying that the data maintained their spatial accuracy within the database. Non-projected data required individually manual re-projection rather than being processed with the known projected data. Due to post processing prior to this project, it was unclear what datum some of the data actually possessed. Projecting and editing the data took approximately 80 hours to finalize and was finished in April 2016.

Initially, the database design comprised five divisions, each representing a different geographic mesa and outliers, with data representing similar features from different sites merged together. However, input data contained multiple attribute schemas, making it difficult to comply with the original task within the time constraint; thus, the scope was reduced. The contingency plan separated the database by archaeology sites, rather than mesa, and efforts to normalize the data were implemented. The geodatabase design was 
set back by one month and not completed until May 29, 2016 due to the change in the logical design and the additional steps involved in data scrubbing.

Originally, all symbols were to be hand-drawn in Inkscape 0.91 before uploading the designs into ArcMap 10.3. While designing the point symbols was uncomplicated, designing the polygon and line patterns proved to be difficult due to the obvious repeating patterns and were later created in ArcMap using the Symbol Properties Editor. Creating and validating the symbol set took a month longer than allotted in the original project plan; the foreseen end date in the original project plan was in April, and the actual completion date was in May. Designing, creating, and importing the symbols into ArcMap took approximately 70 hours to complete since the client reviewed multiple prototypes. Due to this setback, producing the map series was postponed by another month but still completed in late June by increasing the hours of labor. It took approximately one hour to make two to four maps and approximately 80 hours to finalize the map series.

Initially, all quality control was scheduled for June, but testing the symbology required more steps than first assumed and started in April. An online survey provided a method for testing the functionality and intuitiveness of the custom symbology in relation to the original symbology with University of Redlands undergraduate and graduate student participants enrolled in archaeology, anthropology, history, environmental science, or GIS. To administer the survey, the Collaborative Institutional Training Initiative (CITI) training for human subject testing needed to be completed and then attached to the Institutional Review Board (IRB) application for approval. The CITI training contained 16 lesson models that each took about an hour to complete; the certificate was received April 26, 2015. The IRB approved the form and sent it to Student Life for supplemental consent on May 6, 2016. The survey launched May 23, 2016 via email though an anonymously accessible link. Figure 3-3 compares the original project plan to the final plan. 


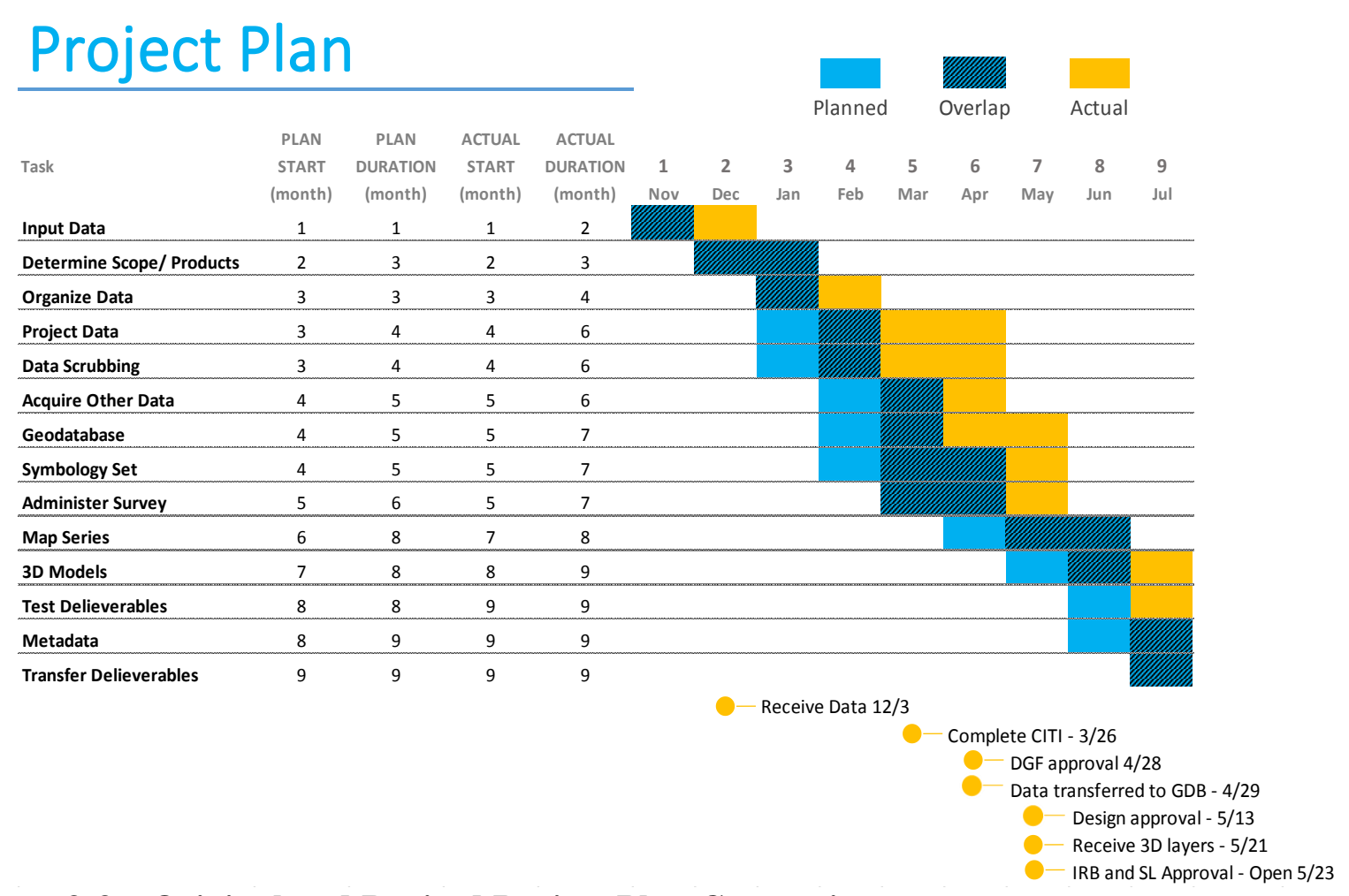

Figure 3-3: Original and Revised Project Plan Comparison.

As the project progressed, identifying what additional tasks required extra hours of labor became more apparent. Though the schema of the original project plan comparatively matched the revised version, there were two major differences. First, the original plan miscalculated the time needed to complete each task and was edited in the revised project plan. Second, the tasks overlapped during the actual development phase rather than completing each task individually as first assumed in the original plan.

\subsection{Summary}

This project provided a unique and uniform means of visualizing archaeology sites on the Hopi reservation. The original project plan assumed the hours of labor needed to complete each task, but the allotted hours needed revision as the project progressed. The final products included a spatial database, a custom symbol set, a map series, and 3D models to improve existing maps for future publications. Accessing the University of Redlands Spatial Studies server provided a stable means of acquiring the input data, as well as an efficient way of transferring the final deliverables to the client, which he can access from his desktop. Most of the final products were cartographic solutions that did not require a major system design, but the database, symbol set file, and final digital maps required a significant amount of planning to satisfy predefined requirements and be available for the client through the server. 



\section{Chapter 4 - Database Design}

Over the past decade, the client stored site survey data as individual shapefiles in Windows Explorer. This led to many inconsistencies as data collection methods evolved over the years. Producing a spatial geodatabase organized existing data and fulfilled the client's requirement to transfer the input data into a more systematic database. Section 4.1 discusses the conceptual data model to reveal the relationships found between realworld features on the Hopi reservation and explains how these relationships underpinned the final database design. Section 4.2 explains the logical data model of the spatial database, as well as outlines the feature and attribute schemas of the data. This session also notes how the logical data model evolved during the project. Section 4.3 covers the sources data provided by the client and the Digital Globe Foundation and explains why these datasets were used. This section also discusses data modifications prior to this project. Section 4.4 discusses the necessary data scrubbing and loading phase with the data provided by the client. Section 4.6 concludes the chapter by highlighting some of the key components and major milestones in completing the database design.

\subsection{Conceptual Data Model}

The Hopi Reservation covers a roughly 2,500 square mile area in northeastern Arizona, with 48 medium to large archaeological sites spread across approximately 1,270 square miles. The majority of excavation sites reside on or near four geographic plateaus - First Mesa, Second Mesa, Third Mesa, and Antelope Mesa - in the northeastern area of the reservation. Archaeological boundaries consist of three levels, which include surveys, site boundaries, and archaeological sites. Survey sites surround site boundaries that contain archaeological sites, but not all archaeological sites are within a survey. For example, the Dawake Survey boundary surrounds multiple site boundaries denoted by an archaeological catalog number. These site boundaries may or may not contain archaeological sites. Most site boundaries have catalog numbers to identify each site written as North Arizona followed by an integer (i.e. NA1699). Survey names identify the survey areas. Larger archaeological sites are usually independent from site or survey boundaries, while smaller excavation efforts are within these archaeological borders. The site name and catalog number identify the site.

Archaeological features make up archaeological sites and are separable into three categories: artifacts, structures, and excavations. Artifacts are archaeological remains left by the Hopi people and separated into two groups. Discrete objects found by archaeologists include distinct features such as rock art, hearths, or isolated occurrences (pottery or arrowheads). Area ruins consist of middens, zones of shattered debris or trash deposit, or an area of large rubble indicating habitation. Structures include edifices like walls, pithouses, and shrines. Observable structures are evident habitation features found during fieldwork while inferred structures are theorized wall alignments based on patterns found in rubble. Lastly, excavation features classify legal or illegal dug features created during digs. Archaeologists sanction areas, such as a survey site, to perform legal excavation digs and station collection units. Illegal excavations performed by looters entail potholes and defacing to remove discrete artifacts from the site. Dr. Bernardini originated these archaeological feature data through years of fieldwork, and these 
provided data determined the logical model structure. Figure 4-1 depicts the conceptual model.

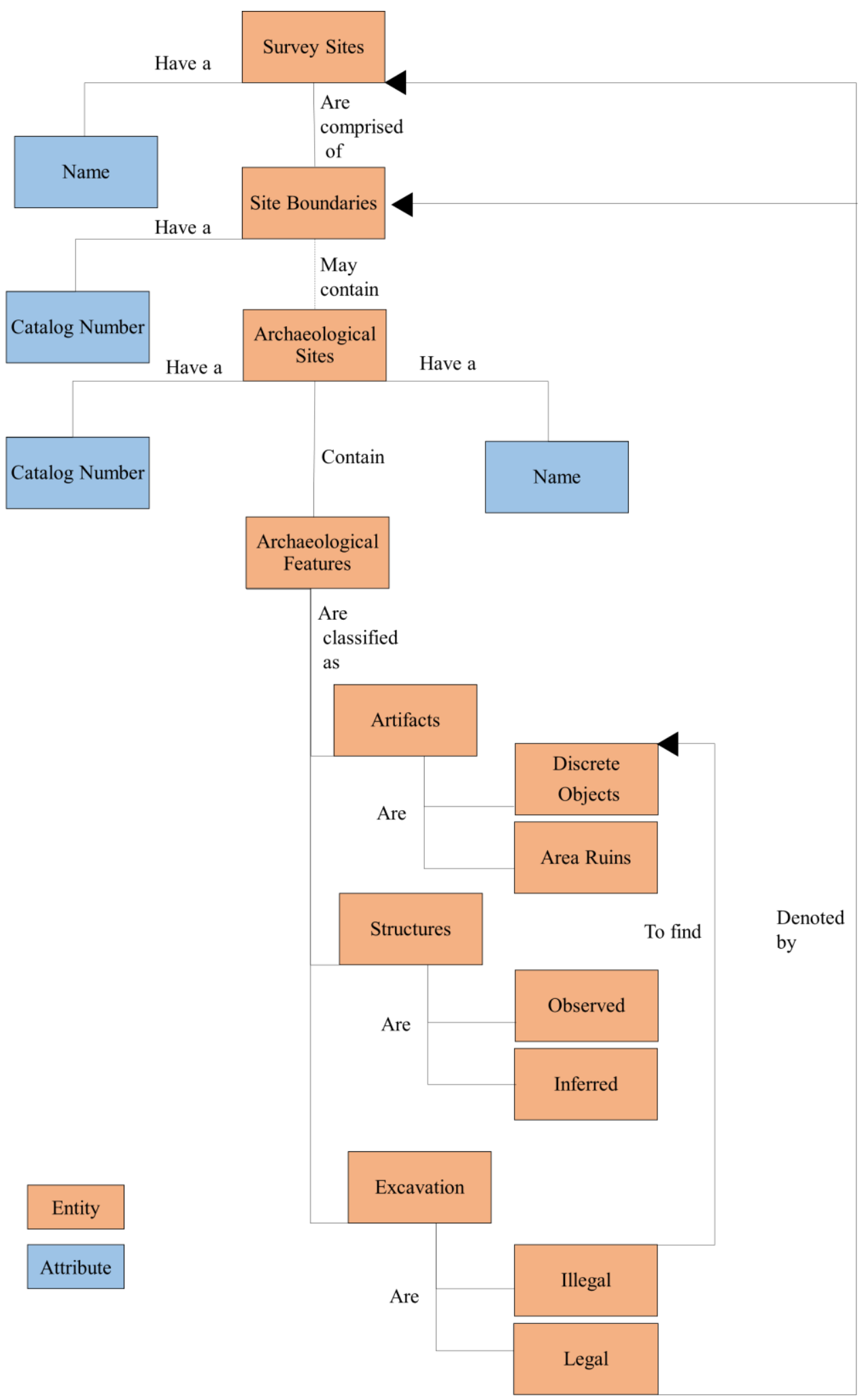

Figure 4-1: Conceptual Data Model. 


\subsection{Logical Data Model}

All of the shapefiles were transferred to an Esri ArcGIS10.3 File Geodatabase since the client is an ArcGIS user. The input data were collected at archaeological site level and contained five survey sites. Each Widows Explorer file denoted an archaeological site and contained a series of shapefiles pertaining to the particular site. Having such a substantial amount of shapefiles intermingled with other work and stored in multiple folders created unnecessary clutter for the client and made data management difficult. Since input data possessed inconsistences, transferring the information into a File Geodatabase standardized the projection system and stored the information in a more systematic format. The geodatabase is also more versatile, allowing the client to add, edit, and remove data in the database and relocation data to new locations more efficiently.

Since sites lie on or near four mesas, the geographical location of the sites along these regions characterized the first proposed logical model. Figure 4-2 depicts a contents diagram illustrating where archaeological sites are located within the reservation. The first proposed logical database model mimicked this relationship by dividing the archaeological sites into five feature datasets. Four feature datasets represented the four mesas and one represented the compiled outliers (Figure 4-3).

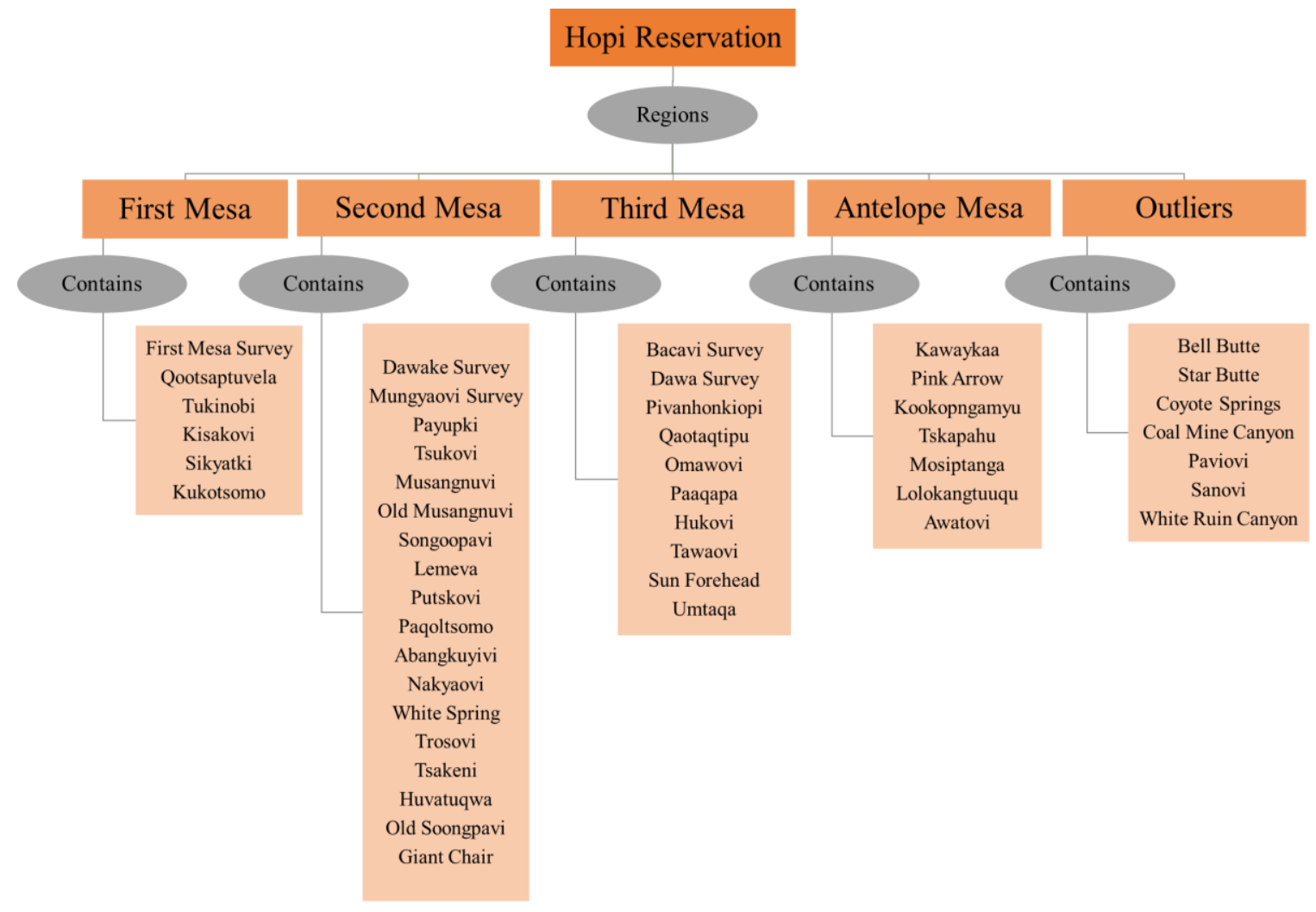

Figure 4-2: Contents Diagram of Sites within the Hopi Reservation.

The feature datasets contained feature classes of each type of archaeological feature found on that mesa by combining the similar shapefiles. For instance, all of the Second Mesa collection units would be amalgamated as one feature class rather than multiple 
shapefiles containing points for collection units on each archaeological site. By combining all of the collection units, the one feature class would increase the integrity of the data and spatial analyses could be performed for the entire mesa rather than repeating the process multiple times for each site.

These fields included one for the site name titled Site_Name, one for the archaeological catalog number titled NA_number, and a unique identifier titled Source_ID. Though the majority of boundary sites possess catalog numbers, some sites had duplicating numbers or did not have a number associated with them. Therefore, each site or survey received a unique identifier noting the mesa in which they are located and a number (i.e. FM1, SM9, TM2, AM6 and OL5). From there, similar features could be merged together but retain the information about where a particular object originated. Figure 4-3 illustrates the original logical data model.

However, merging the input data was more tedious than expected, and it became apparent that designing the first proposed data model did not match the existing data structure. Collection methods gathered data at the archaeological site level, so merging data by geographical region called for a new system design.

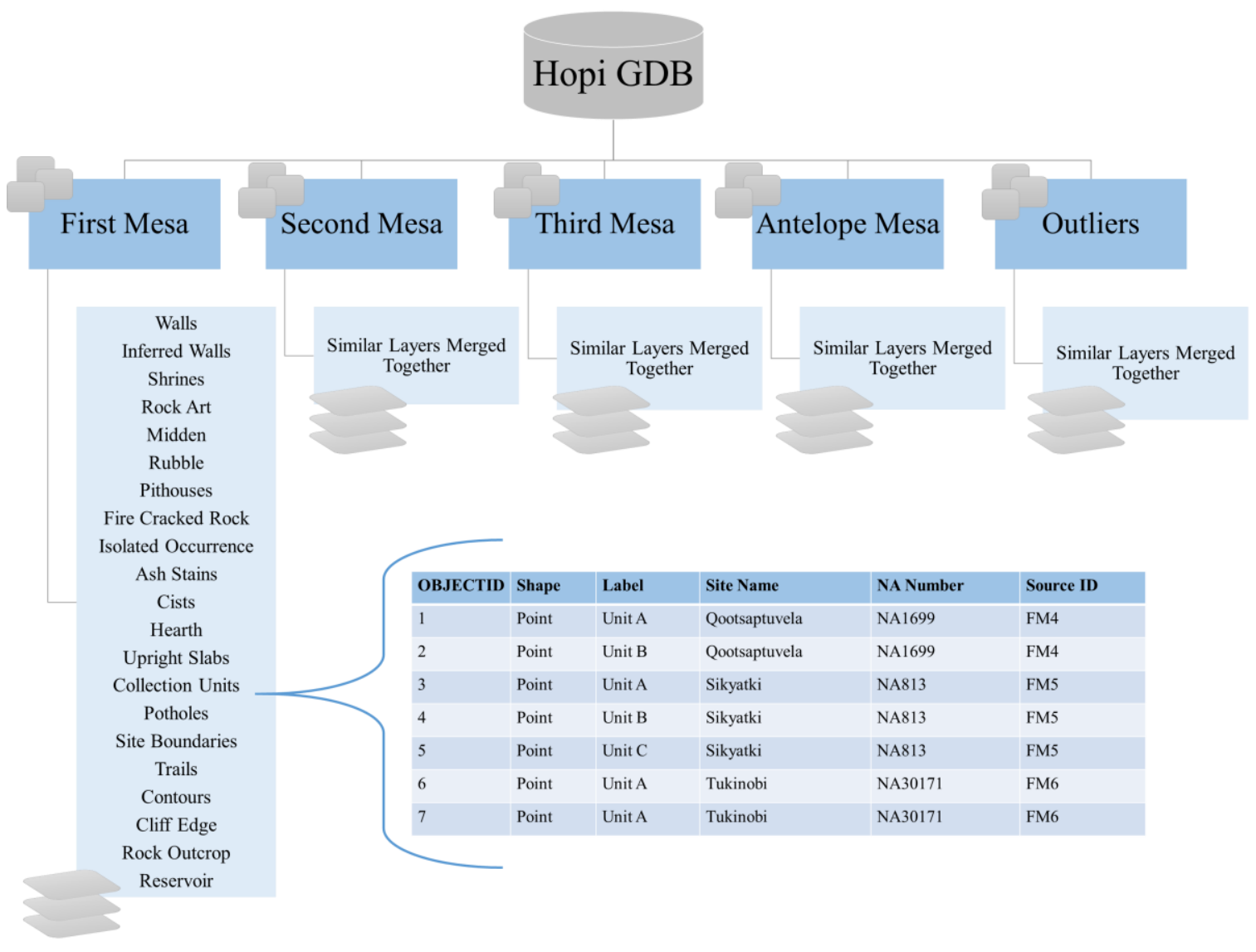

Figure 4-3: Original Logical Data Model. 
It was assumed that similar features maintained consistent attribute schemas; however, since data collection methods changed over time, so too did the attribute schemas. Even after adding the Site_Name, NA_number, and Source_ID fields to the feature class attribute tables, the feature classes merged incorrectly due to the multiple attribute schemas. In Figure 4-4, examples illustrate the various attributes schemas found in the input data to record collection units.

All of the attribute tables for collection units contained fields noting the Northing and Easting coordinates and the elevation. However, data about unit titles varied.

Examples (a) and (c) contain unit title information in the Desc_field while examples (b), (d), and (e) use the Unit field. Note the various ways for recording title information; (a) uses all capital letters, (b) uses a capital letter, (c) abbreviates collection units, and (d) and (e) use sentence structure. The final map series labeled the collection units, so the edited fields matched the sentence structure version. Additional inconsistences include fields for collection unit diameter, GPS collection date, photos, data files, and commentary.

Because input data contained multiple attributes schemas, combining similar features through the Merge geoprocessing tool in ArcMap became unfeasible. The tool did not identify what fields were, or were not, included in each attribute schema to merge the corresponding fields together. Moreover, fields with the same name incorrectly merged even though the cells did not contain similar information. In the final logical model, the redesigned geodatabase represents data at the archaeological site level. 


\begin{tabular}{|c|c|c|c|c|c|c|c|}
\hline \multicolumn{8}{|c|}{ NA1699_Collection_Units } \\
\hline & OB.JECTID * & Shape * & Point & Northing & Easting & Elevation & Desc \\
\hline & 1 & Point ZM & 0 & 3965213 & 554123 & 1808 & UNIT A \\
\hline & 2 & Point ZM & 0 & 3965219 & 554116 & 1803 & UNIT B \\
\hline & 3 & Point ZM & 0 & 3965297 & 554212 & 1805 & UNIT C \\
\hline & 4 & Point ZM & 0 & 3965369 & 554235 & 1814 & UNIT D \\
\hline & 5 & Point ZM & 0 & 3965305 & 554178 & 1818 & UNITE \\
\hline & 6 & Point ZM & 0 & 3965373 & 554249 & 1814 & UNIT F \\
\hline & 7 & Point ZM & 702 & 3965168.65466 & 554163.53749 & 1835.72791 & UNIT G \\
\hline
\end{tabular}

(a)

\begin{tabular}{|c|c|c|c|c|c|c|c|c|c|c|c|}
\hline \multicolumn{12}{|c|}{ NA30661_Collection_Unit } \\
\hline & OBJECTID * & Shape * & Unit & Diameter & Comment & Pic1 & GPS_Date & Datafile & GNSS_Heigh & Northing & Easting \\
\hline \multirow[t]{4}{*}{1} & 1 & Point ZM & A & $1 \mathrm{~m}$ & & & $6 / 26 / 2014$ & H062613A.cor & 1963.986 & 4002293.773 & 541047.44 \\
\hline & 2 & Point ZM & B & $1 \mathrm{~m}$ & & & $6 / 26 / 2014$ & H062613A.cor & 1960.958 & 4002273.173 & 541039.908 \\
\hline & 3 & Point ZM & C & $1 \mathrm{~m}$ & & & $6 / 26 / 2014$ & H062613A.cor & 1960.187 & 4002242.124 & 541019.27 \\
\hline & 4 & Point ZM & D & $1 \mathrm{~m}$ & & & $6 / 26 / 2014$ & H062613A.cor & 1971.671 & 4002301.959 & 540982.705 \\
\hline
\end{tabular}

(b)

NA1169_Collection_Unit
\begin{tabular}{|r|r|c|r|r|r|r|l|}
\hline & \multicolumn{1}{|c|}{ OBJECTID * $^{*}$} & Shape * & POINT & \multicolumn{1}{c|}{ NORTHING } & \multicolumn{1}{c|}{ EASTING } & ELEVATION & DESC_ \\
\hline 1 & Point ZM & 39 & 3971740.6752 & 526840.10825 & 1920.65135 & CU \\
\hline & 2 & Point ZM & 77 & 3971734.24649 & 526856.64064 & 1920.38847 & CU \\
\hline & 3 & Point ZM & 142 & 3971717.95517 & 526902.77209 & 1889.87508 & CU \\
\hline & 4 & Point ZM & 171 & 3971734.70915 & 526912.12868 & 1890.85749 & CU \\
\hline & 5 & Point ZM & 181 & 3971750.24373 & 526907.45016 & 1898.45512 & CU \\
\hline & 6 & Point ZM & 182 & 3971746.96697 & 526915.49396 & 1894.30535 & CU \\
\hline
\end{tabular}

(c)

\begin{tabular}{|c|c|c|c|c|c|c|c|c|c|c|c|}
\hline \multicolumn{12}{|c|}{ NA30661_Collection_Unit } \\
\hline & OBJECTID * & Shape * & Unit & Diameter & Comment & Pic1 & GPS_Date & Datafile & GNSS_Heigh & Northing & Easting \\
\hline & 1 & Point ZM & Unit A & $1 m$ & & & $6 / 26 / 2014$ & H062613A.cor & 1963.986 & 4002293.773 & 541047.44 \\
\hline & 2 & Point ZM & Unit B & $1 \mathrm{~m}$ & & & $6 / 26 / 2014$ & H062613A.cor & 1960.958 & 4002273.173 & 541039.908 \\
\hline & 3 & Point ZM & Unit C & $1 \mathrm{~m}$ & & & $6 / 26 / 2014$ & H062613A.cor & 1960.187 & 4002242.124 & 541019.27 \\
\hline & 4 & Point ZM & Unit D & $1 \mathrm{~m}$ & & & 6/26/2014 & H062613A.cor & 1971.671 & 4002301.959 & 540982.705 \\
\hline
\end{tabular}

(d)

\begin{tabular}{|c|c|c|c|c|c|c|c|c|c|c|}
\hline \multicolumn{11}{|c|}{ FMS_Collection_Units } \\
\hline & OBJECTID* & Shape * & Id & Unit & Label & Pic_1 & Pic_2 & Feat_Name & Datafile & Data_Dicti \\
\hline , & 1 & Point ZM & 0 & Unit A & & & & & & \\
\hline & 2 & Point ZM & 0 & Unit B & & & & & & \\
\hline - & 3 & Point ZM & 0 & Unit C & & & & & & \\
\hline & 4 & Point ZM & 0 & & unit a & & & Collecti & WRB061211A.cor & Mapping Dictionary \\
\hline
\end{tabular}

(e)

Figure 4-4: Attribute Schema Inconsistencies for Collection Unit Data.

Figure 4-5 depicts a simplified model of the revised geodatabase design. The File Geodatabase houses 48 feature datasets representing each archaeological site with each feature dataset contains the archaeological features as feature classes. Surveys site were represented as feature datasets while the site boundary data were included as a feature class with the archaeological features. Using this design allows the client to select a feature dataset and open all of the relevant feature classes to visualize one site. However, this design limits the representation of spatial relationships between sites. An analysis tool must be run multiple times for different sites using the same variables rather than processing the data at once under one tool. 


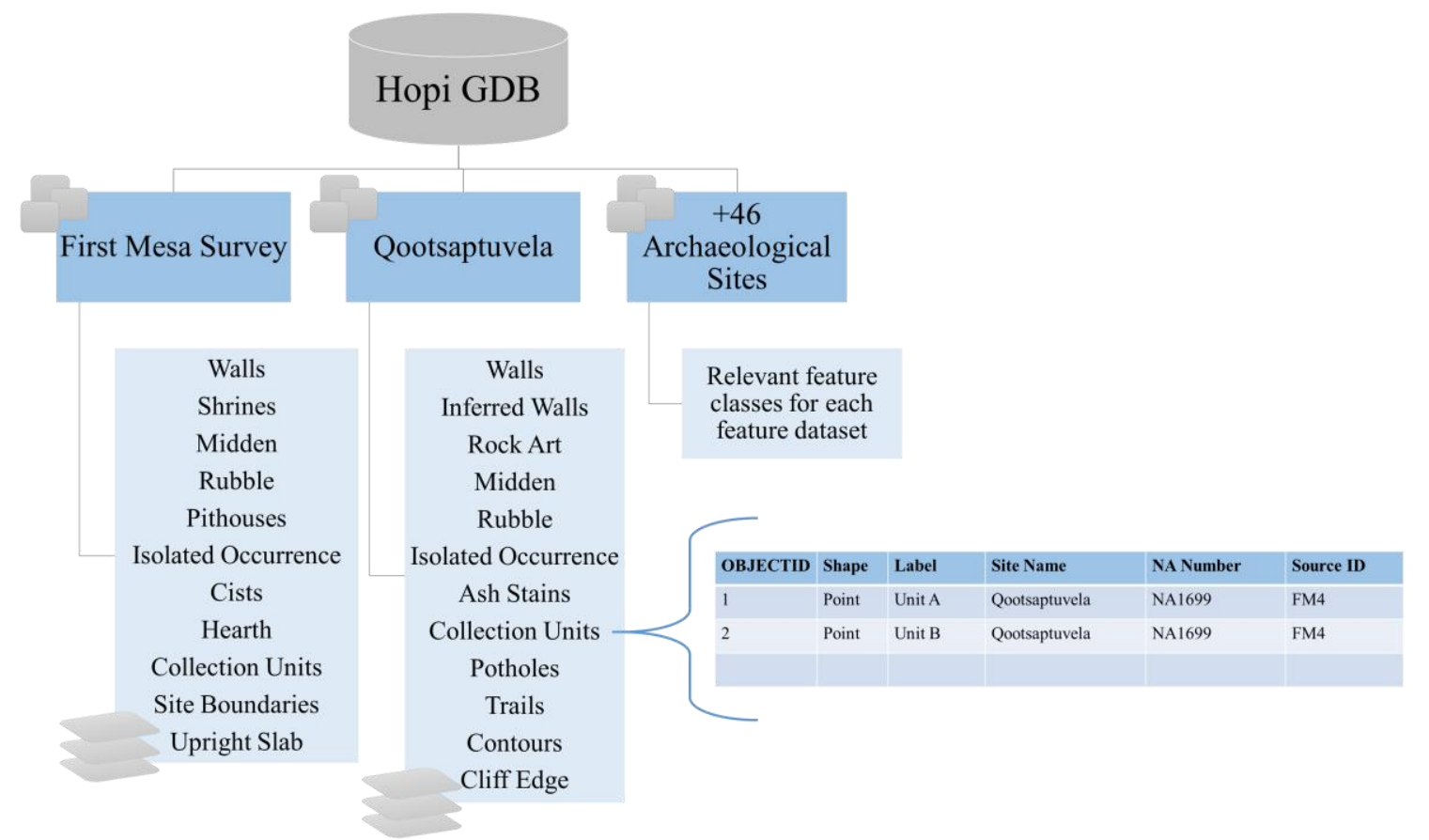

Figure 4-5: Revised Logical Data Model.

In summary, the final geodatabase modeled around the conceptual model starting at the archaeological site level. The three fields that recorded site name (Site_Name), archaeological catalog number (NA_number), and unique identifier (Source_ID) added to the legacy of each feature class within the geodatabase. However, due to the inconsistent attribute schemas previously created, merging similar data was beyond the scope of this project. The data received a standardized projection system and efforts to maintain the integrity of the data continued by consolidating the features classes within the geodatabase.

\subsection{Data Sources}

The client provided archaeological data collected using a GPS unit during personal fieldwork for the past decade; these data were stored in Esri shapefile format. These data included the survey boundaries and individual features found on each site (i.e. walls, rubbles, or collection units). Upon delivery, the client provided $22 \mathrm{~GB}$ worth of data comprised of survey photographs, non-referenced aerial imagery, shapefiles, prior maps, and ArcMap and ArcScene files. However, not all of the input data were necessary for this project. Shapefiles were separated from unnecessary data such as non-georeferenced images, photos, and Excel documents. There was 595 shapefiles (excluding test and unnecessary shapefiles) for this project, and no metadata existed within the shapefiles.

The Image Finder search tool on the Digital Globe Foundation website located the imagery strips and provided each strip's identification information. A generalized study area shapefile containing the site boundaries gave an approximation of the imagery needed. The selected data was $50 \mathrm{~cm}$ panchromatic and multispectral imagery taken from the World View 2 satellite. The requested imagery ranged from 2010 to 2015 since there 
was a limited selection of imagery strips in some areas and some imagery contained high cloud cover percentages.

Figure 4-6 is a snapshot of the Image Finder interface used to select the imagery strips. The red line represents the generalized boundary of the 48 archaeological sites contained on the four mesas and the seven outliers. The black lines represent the imagery options taken by the WorldView 2 satellite, while the strips containing imagery were the requested orthophotography. The yellow highlighted strips note the main area of interest.

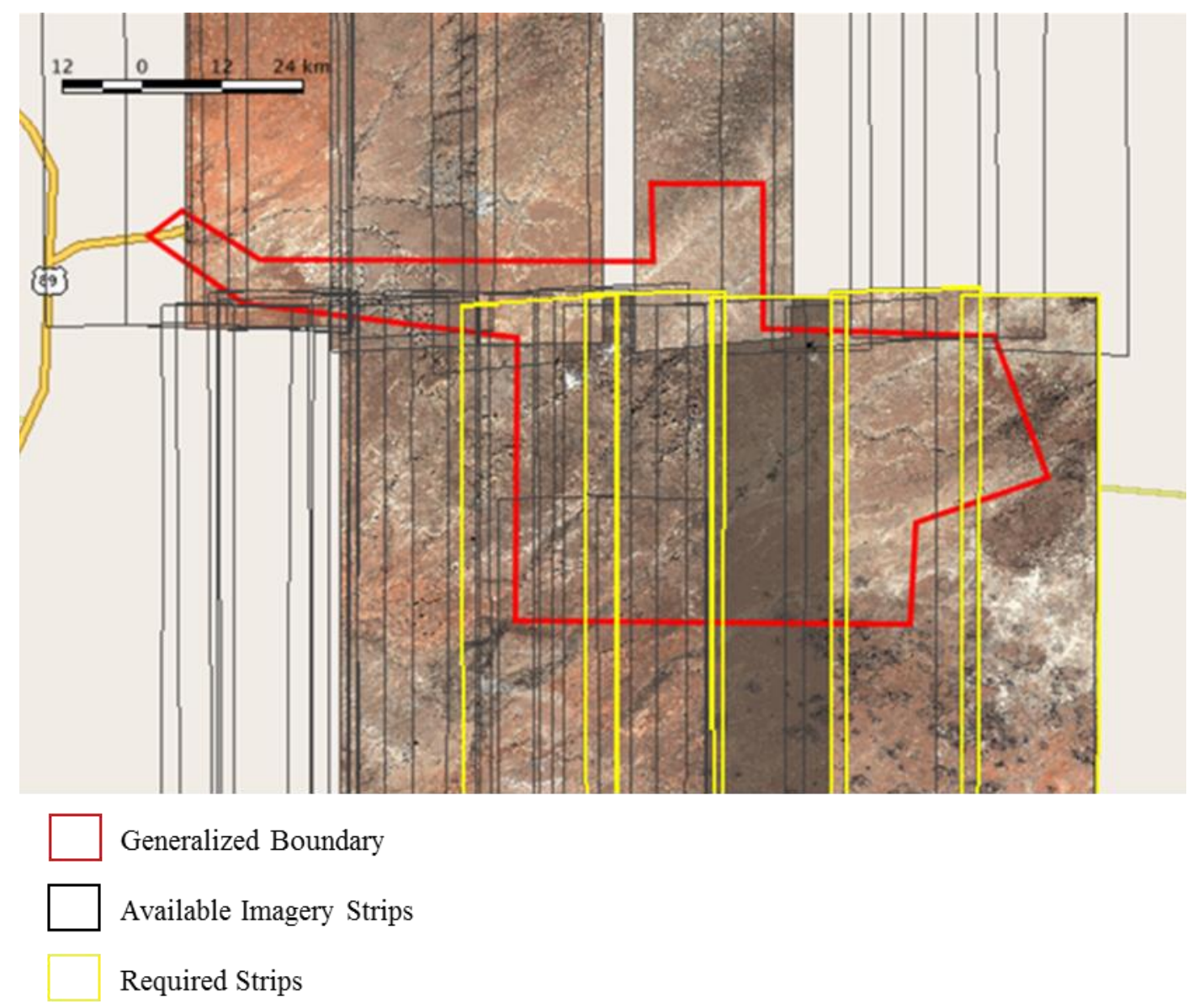

Figure 4-6: Imagery Requested on Image Finder from Digital Globe Foundation. World View 2 satellite $50 \mathrm{~cm}$ resolution imagery (2010-2015).

Unfortunately, no available strips covered one of the major sites in the study area. The Digital Globe Foundation clipped the imagery to the generalized study area shapefile before delivery. Any other outside data collection was unnecessary for this project; the client delivered all the vector data and Digital Globe Foundation provided the imagery.

The Digital Globe Foundation provided $50 \mathrm{~cm}$ resolution imagery for a 1,270 square mile area that encompassed the 48 archaeological sites after applying and receiving approval for the high-resolution orthophotography. Imagery was downloaded in Tagged Image File Format (TIFF). The imagery provided a means of visually testing the positional accuracy of the archaeological data before and after standardizing the projection and generated detailed orthophotography landscapes for the 3D models. While 
the Digital Globe Foundation website provided attribute information about the imagery, there were no metadata provided with the files.

\section{Table 2. Image Strips Requested from Digital Globe Foundation.}

\begin{tabular}{|c|c|c|c|c|c|c|c|c|c|}
\hline \multicolumn{10}{|c|}{ Digital Globe Request Areas } \\
\hline Catalog ID & Imaging Bands & Spacecraft & $\begin{array}{c}\text { Acquisition } \\
\text { Date }\end{array}$ & \begin{tabular}{|c|}
$\begin{array}{c}\text { Total Max } \\
\text { Off Nadir } \\
\text { Angle }\end{array}$ \\
\end{tabular} & \begin{tabular}{|c|}
$\begin{array}{c}\text { Area Max } \\
\text { Off Nadir } \\
\text { Angle }\end{array}$ \\
\end{tabular} & \begin{tabular}{|c|} 
Area Min \\
Sun \\
Elevation
\end{tabular} & $\begin{array}{c}\text { Area Max } \\
\text { GSD }\end{array}$ & \begin{tabular}{|c|}
$\begin{array}{c}\text { Total } \\
\text { Cloud } \\
\text { Cover Pct }\end{array}$ \\
\end{tabular} & $\begin{array}{r}\text { Area } \\
\text { Cloud } \\
\text { Cover Pct }\end{array}$ \\
\hline \multicolumn{10}{|l|}{ Data Required } \\
\hline 103001001BACE700 & Pan-MS1-MS2 & WV02 & $8 / 10 / 2012$ & 13.9 & 13.16 & 66.79 & $0.49 \mathrm{~m}$ & $0 \%$ & $0 \%$ \\
\hline $103001001 \mathrm{~A} 694500$ & Pan-MS1-MS2 & WV02 & $8 / 10 / 2012$ & 24.45 & 24.03 & 66.64 & $0.55 \mathrm{~m}$ & $0 \%$ & $0 \%$ \\
\hline $103001001 \mathrm{~B} 328200$ & Pan-MS1-MS2 & WV02 & $9 / 25 / 2012$ & 3.16 & 1.71 & 52.19 & $0.46 \mathrm{~m}$ & $9 \%$ & $0 \%$ \\
\hline $103001001 C 075600$ & Pan-MS1-MS2 & WV02 & $9 / 25 / 2012$ & 13.73 & 13.73 & 52.03 & $0.49 \mathrm{~m}$ & $7 \%$ & $0 \%$ \\
\hline 103001004 CA9B000 & Pan-MS1-MS2 & WV02 & $12 / 3 / 2015$ & 22.55 & 22.55 & 30.76 & $0.54 \mathrm{~m}$ & $0 \%$ & $0 \%$ \\
\hline \multicolumn{10}{|c|}{ Additional Data Request } \\
\hline $103001004 F 1 A 5100$ & Pan-MS1-MS2 & WV02 & $1 / 21 / 2016$ & 20.97 & 19.28 & 31.11 & $0.51 \mathrm{~m}$ & $0 \%$ & $0 \%$ \\
\hline $103001003 F 2 C 4100$ & Pan-MS1-MS2 & WV02 & $4 / 14 / 2015$ & 23.65 & 23.65 & 59.26 & $0.54 \mathrm{~m}$ & $0 \%$ & $0 \%$ \\
\hline $103001000896 \mathrm{~B} 300$ & Pan-MS1-MS2 & WV02 & $12 / 11 / 2010$ & 21.2 & 21.04 & 30.18 & $0.53 \mathrm{~m}$ & $0 \%$ & $0 \%$ \\
\hline $103001000 C 872200$ & Pan-MS1-MS2 & WV02 & $6 / 7 / 2011$ & 12.16 & 12.16 & 74.3 & $0.48 \mathrm{~m}$ & $0 \%$ & $0 \%$ \\
\hline $103001004079 \mathrm{BB} 00$ & Pan-MS1-MS2 & WV02 & $4 / 14 / 2015$ & 20.73 & 20.73 & 59.44 & $0.52 \mathrm{~m}$ & $0 \%$ & $0 \%$ \\
\hline 1030010046720000 & Pan-MS1-MS2 & WV02 & $8 / 6 / 2015$ & 24.44 & 23.62 & 63.04 & $0.54 \mathrm{~m}$ & $0 \%$ & $0 \%$ \\
\hline 103001004 C8FCF00 & Pan-MS1-MS2 & WV02 & $11 / 6 / 2015$ & 16.86 & 14.25 & 36.68 & $0.49 \mathrm{~m}$ & $0 \%$ & $0 \%$ \\
\hline
\end{tabular}

\subsection{Data Scrubbing and Loading}

Due to many inconsistencies, the archaeological input data required a substantial amount of data scrubbing. GIS has changed significantly over the past decade, and since the client collected fieldwork data over the past ten years, the data reflected these differences. Data management and formatting, for example, changed drastically from individual features being stored separately as shapefiles to data more systematically managed within a spatial database. Archaeological fieldwork collection methods also evolved over time, and as a result, attribute information for similar features began to vary with each excavation site. Because of the inconsistencies found in the attribute schemes, organizing the data in the originally conceived logical model became out of scope, and the logical model needed revision.

To determine the necessary data, the visible layers within the Table of Contents panel in original ArcMap files denoted the data to export into the geodatabase and use in the map series; the turned-off layers were unnecessary for the project. However, not all of the pathways connected between the data and the original ArcMap files when using the Spatial Studies server, so the pathways between the data and the matching layer were manually reconnect. Due to inconsistent and vague naming conventions, it was difficult to determine what data matched the maps layer, but the client received prototype geodatabases and provided insight on which layers were required in the geodatabase. Once the geodatabase design was complete, the data were given a new naming scheme for a more visually cohesive and navigable geodatabase. The feature datasets included the site name and unique identifier for the archaeological site while the feature classes contained the archaeology catalog number (Figure 4-7). 


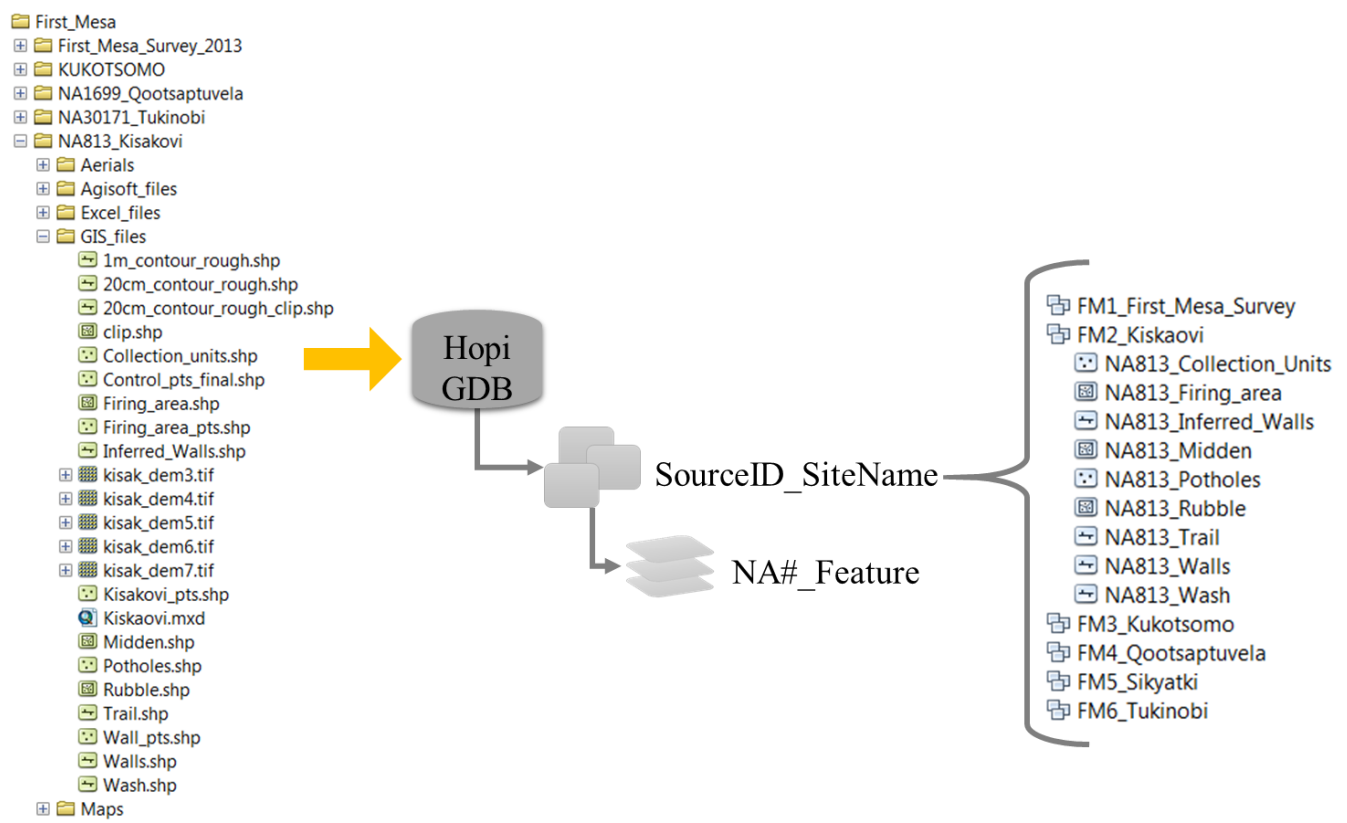

Figure 4-7: Geodatabase Naming Schema.

The input data used a variety of projection systems that included NAD27 UTM Zone12, World Geodetic System, and unknown spatial references. Therefore, the client chose a single projection system for the data to be re-projected (NAD 83 UTM Zone 12). Using the Batch Projection geoprocessing tool, the function converted all of the data except for those with an unknown spatial reference. Data without a projection system were changed manually since the computation from one projection system to another could not be performed on non-projected data.

Unfortunately, prior processing on the input data interchangeably used two geoprocessing tools to alter the projection: Project and Define Projection. One tool changes the projection system and shifts the data based on that projection, while the other simply redefines the current projection system. While the Sources tab stated that they were in NAD83 UTM Zone 12, manual checks revealed that some of the data were possibly in the NAD 27 datum due to prior geoprocessing. However, the absence of metadata made it difficult to determine any previous data processing. Some data shifted incorrectly after standardizing the projection system, so the client agreed to help manually shift the spatially inaccurate data back to their appropriate positions in order to move forward to the development phase.

\subsection{Summary}

Though shapefiles efficiently transfer data to new locations, storing data within a geodatabase maximizes the functionality and integrity of data. Prior to this project, the client stored fieldwork data as shapefiles, but over the last decade, this format became unsuitable and there became a need for a geodatabase. The conceptual model illustrates the relationship between archaeological features and their location on the Hopi reservation and provides the basis for constructing the logical model for the File Geodatabase. The end geodatabase compiled the spatial data needed for this project into 
48 feature datasets, each containing fieldwork data from one excavation site, and although a previous logical schema existed, the inconsistencies contained within the input data challenged that original design. Data sources and data collection methods included the client's data, as well as high-resolution orthophotographic imagery from the Digital Globe Foundation. 



\section{Chapter 5 - Implementation}

This chapter provides an in-depth discussion of the components implemented to design the final products for this project. Section 5.1 revisits the geodatabase design and development, as well as the inconsistencies found within the input data that set the cartographic workflow tasks back in the project plan. Section 5.2 discusses the custom symbology set creation process and the efforts to implement the symbology into Esri software. Section 5.3 covers the map series created using the custom symbology and the design choices selected for the maps. Section 5.4 addresses how the 3D models rendered, as well as how this aspect of the project evolved during the development phase.

\subsection{Data Processing}

Before designing the other components, the data required scrubbing and migration to a more manageable format. The input data used multiple projection systems, and the client wanted the data in a standardized format. Visually, the layers overlaid incorrectly and the data required editing before continuing to the cartographic tasks in the workflow.

The data necessary for this project were separated from the input data by opening the ArcMap 10.3 and ArcScene 10.3 files and exporting the visible layers into the geodatabase. Non-connected pathways were linked manually with the correct files in Windows Explorer. This was a setback since test data and unclear names were given to files intermingled with the required data. The geodatabase imported the shapefiles as feature classes and re-projected in NAD83 UTM Zone12. The client received prototype versions of the geodatabase to ensure the database contained the data for the map series. The database comprised of 48 feature datasets representing each archaeological site and contained feature classes of objects located on each site. To confirm that all of the data received a standardized projection system, the Batch Project geoprocessing tool tested whether the geodatabase transformed the input data.

The results showed that some data possessed an unknown spatial reference and needed manually changes since the geoprocessing tool could not adjust the nonreferenced data. Once the metadata confirmed that the data were projected in NAD83, a visual check of the data showed that some data shifted incorrectly to the new datum. Some data aligned to the NAD27 datum while the majority of data positioned accurately in NAD83. This data needed to be manually shifted to their accurate locations with the help of the client and visually checked with the Digital Globe Foundation and Esri imagery. Figure 5-1 shows the positional inaccuracy of features on Qootsaptuvela after adjusting to the NAD83 datum. The left image shows all of the layers located 60 meters in the northeastern direction except for the rock art layer (blue points). The right image shows the data manually edited to the appropriate locations along the edge of the cliff face. 


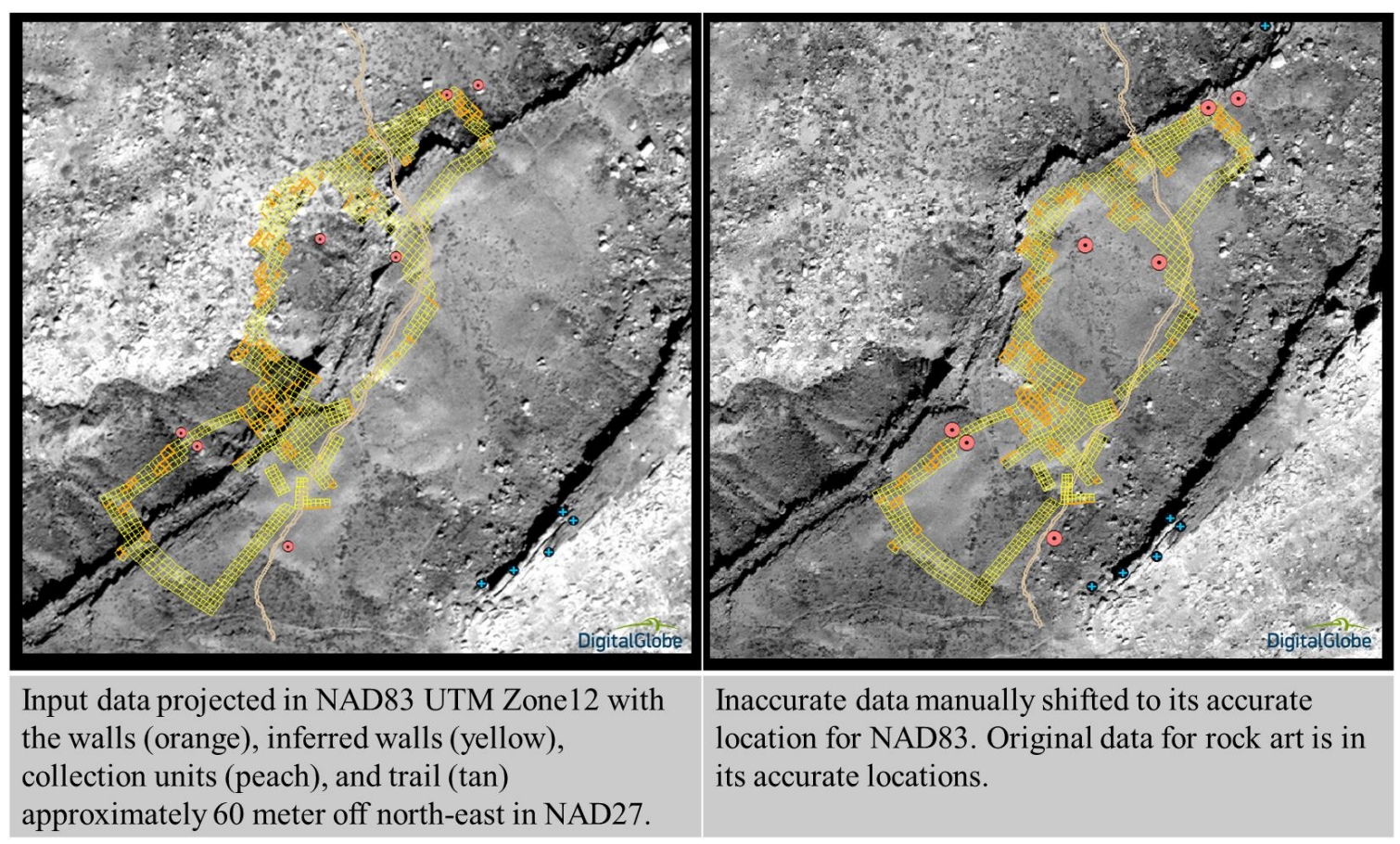

Figure 5-1: Position Inaccuracy after Projecting Input Data.

The data compiled into a geodatabase received unique names, so the data possessed a more consistent naming format. Feature dataset names contained the source ID and site name, SourceID_SiteName, while feature class names contained the archaeological catalog number and the name of the feature, NAnumber_Feature. Amalgamating the data would improve the geodatabase design, even though merging similar feature classes together was not a requirement. This added task, however, became out of scope due to inconsistent attributes schemas among similar features, and only initial steps to begin normalizing the data were implemented. A Python script parsed the new naming schema and populated three newly added fields to the future classes using the ArcPy extension. The script ran through the feature datasets and parsed the unique identifiers and names to populate the Source_ID and Site_Name fields in the attribute tables. The script then iterated through the feature classes and parsed the archaeological number from the name of the feature class to populate the NA_number field.

Only the Mungyaovi survey feature classes were merged since the input data contained approximately 165 shapefiles for the survey site. Though the data were migrated into a geodatabase, navigating though the feature classes was difficult and setting the symbology would be tedious. Instead, allotted time merged the data to produce only 19 feature classes to make the Mungyaovi feature dataset more manageable, totaling the number of feature classes representing archaeological features from 595 to 449 within the geodatabase. For example, the MGS_MNA_Site_Boundaries feature class combined several feature classes for site boundaries, but the polygons are uniquely identifiable by the added NA_number field. Instead of navigating through separate files for each boundary, the client can query a particular site boundary within 
MGS_MNA_Site_Boundaries attribute table. Figure 5-2 represents the workflow for merging the Mungyaovi dataset.

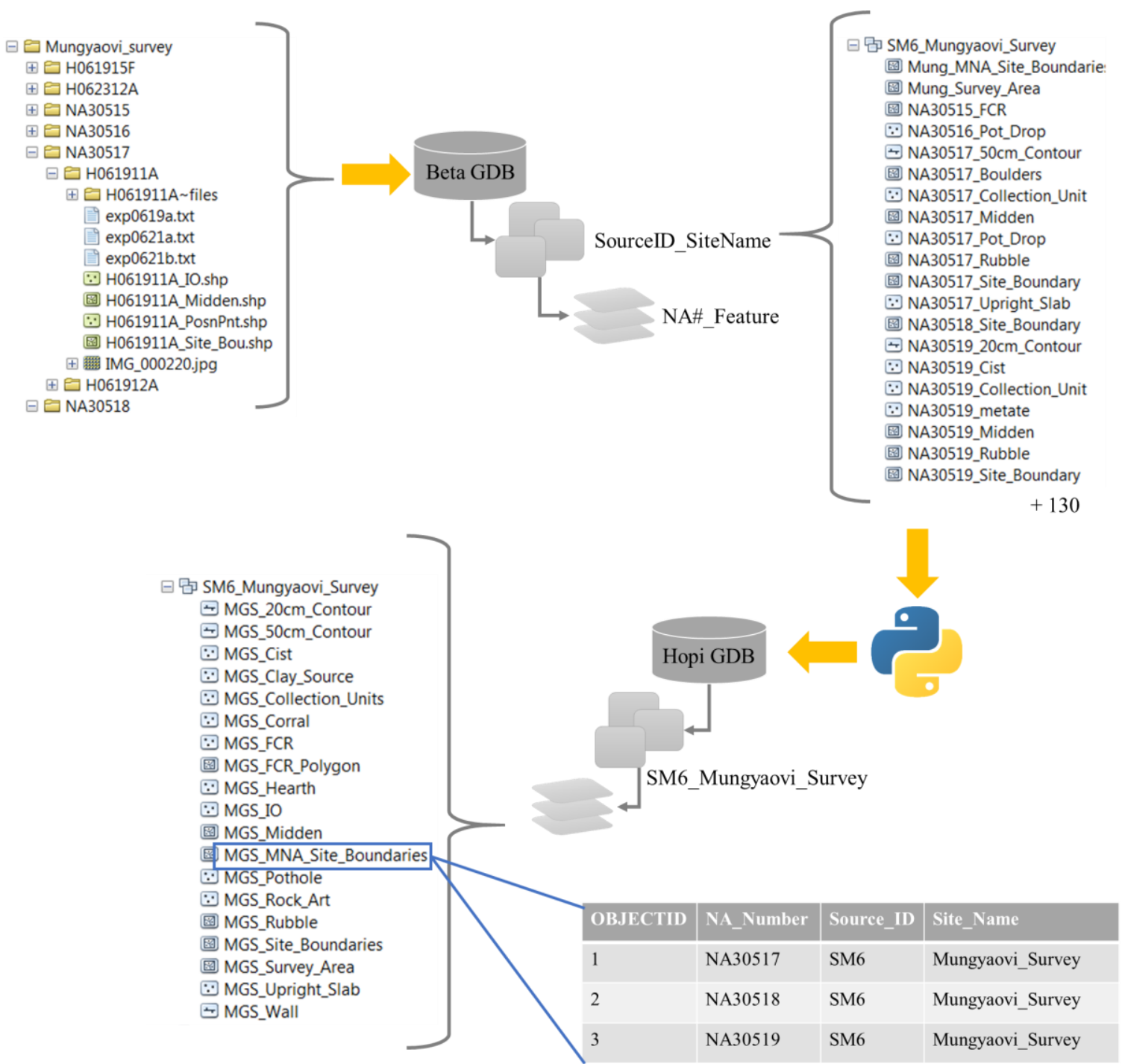

Figure 5-2: Workflow for Merging the Mungyaovi Feature Dataset.

\subsection{Symbology Set}

The client wanted iconic symbols to represent 21 features commonly found on the Hopi reservation. The client provided a list with images and descriptions of each object to provide a basis for the symbol designs, and later with the client, it was determined what vector type represented each archaeological feature. Table 3 provides the list of 21 features and the vector types used to represent the objects. However, there were inconsistencies with the vector type used to represent the data. For example, polygons vectors often represented middens, but older data portrayed middens as line features. For this project, middens were represented using polygon vectors, so the Feature to Polygon geoprocessing tool converted middens portrayed as lines into polygons. Methods for collecting data on cists also evolved over the decade from points to line vectors. 
Cartographically, these inconsistences proved difficult when designing the custom symbology and when determining how to represent the inconsistence without disturbing the standardized look of the maps.

Table 3. Features Required in Custom Symbology Set.

\begin{tabular}{|c|c|c|c|}
\hline & Feature & Description & $\begin{array}{l}\text { Vector Type(s) } \\
\text { Witnessed }\end{array}$ \\
\hline 1 & Cist & A one meter storage feature with circular upright stones & Point, Line \\
\hline 2 & $\begin{array}{l}\text { Collection } \\
\text { Unit }\end{array}$ & A one meter designated radius for locating artifacts & Point \\
\hline 3 & $\begin{array}{l}\text { Fire Cracked } \\
\text { Rock }\end{array}$ & $\begin{array}{l}\text { Small scatter of fire-reddened burned stones that are } \\
\text { often cracked from heat }\end{array}$ & Point \\
\hline 4 & Hearth & A fire pit & Point \\
\hline 5 & $\begin{array}{l}\text { Isolated } \\
\text { Occurrence }\end{array}$ & An individual artifact & Point \\
\hline 6 & Pithouse & $\begin{array}{l}\text { A circular three meter semi-subterranean feature erected } \\
\text { with sticks and mud }\end{array}$ & Point, Line \\
\hline 7 & Pothole & An illegally dug hole for looting & Point \\
\hline 8 & Rock Art & Petroglyphs etched into rock face & Point \\
\hline 9 & Shine & $\begin{array}{l}\text { A one meter religious feature constructed out of stone in } \\
\text { a circle, square, or U shape }\end{array}$ & Point \\
\hline 10 & Upright Slab & Isolated stone slab & Point, Line \\
\hline 11 & Site Boundary & $\begin{array}{l}\text { The archaeological study area with distributed artifacts to } \\
\text { the edges }\end{array}$ & Line, Polygon \\
\hline 12 & Cliff Edge & Edge of a cliff face & Line \\
\hline 13 & Contour & Topographic lines denoting elevation & Line \\
\hline 14 & Inferred Wall & $\begin{array}{l}\text { A theorized wall alignment based on patterns found in } \\
\text { rubble }\end{array}$ & Line \\
\hline 15 & Trail & A dirt road formed by repeated movement along road & Line \\
\hline 16 & Wall & A stone alignment indicating a foundation for a wall & Line \\
\hline 17 & Ash Stain & An ashy surface indicating burned features in sub-terrain & Polygon \\
\hline 18 & Midden & A trash deposit of pottery shards, stone tools, and debris & Line, Polygon \\
\hline 19 & Reservoir & A shallow depression used to collect water runoff & Polygon \\
\hline 20 & Rock Outcrop & Rocky and steep terrain & Polygon \\
\hline 21 & Rubble & Collapsed wall with no indication of wall foundation & Line, Polygon \\
\hline
\end{tabular}

The following requirements were determined for the symbology set:

- Point symbols were created for 10 point or higher size use but are still discernible (or functional) at 9 point or less.

- Marker symbols represented point features.

- Symbology is iconic of Hopi culture or archaeological features. 
- Symbology is unique through shape and color.

- Symbology has color and black versions for different printing options.

- Symbology is reminiscent of traditional stippled archaeology maps.

- Symbol set is selectable within the ArcMap 10.3 Symbology tab.

- Symbology is searchable using tags in the Symbol Selector search bar.

Once the requirements were determined, the next task was to create the symbol designs. Multiple prototype designs were hand-sketched or made in Microsoft PowerPoint and reciprocated to the client until agreed upon for the final designs. All the custom symbology was initially going to be drawn in an imagery processing software called Inkscape 0.91 and imported into ArcMap 10.3, but designing the line and polygon fills by hand became complex. Patterns repeated across large areas with obvious divisions and made the design visually awkward. Therefore, only the point symbology was handdrawn in Inkscape 0.91 and exported in Scalable Vector Graphics (SVG) and Enhanced Metafile (EMF) file formats. ArcMap10.3 uploaded the EMF files as marker symbols. The EMF file maintains graphic dimensions when printing regardless of dots-per-inch, so the image retains its detail at all scales. Line and polygon vectors were designed in ArcMap 10.3 Symbol Property Editor.

All of the symbols were individually saved in the Style Manager in a .style format titled Hopi Archaeology Set. The Hopi Archaeology Set file is viewable in the Symbol Selector interface under the Properties in ArcMap 10.3. The map series used the custom symbology by selecting the symbols in the Symbol Selector interface. Figure 5-3 diagrams the workflow for designing the symbology set. 


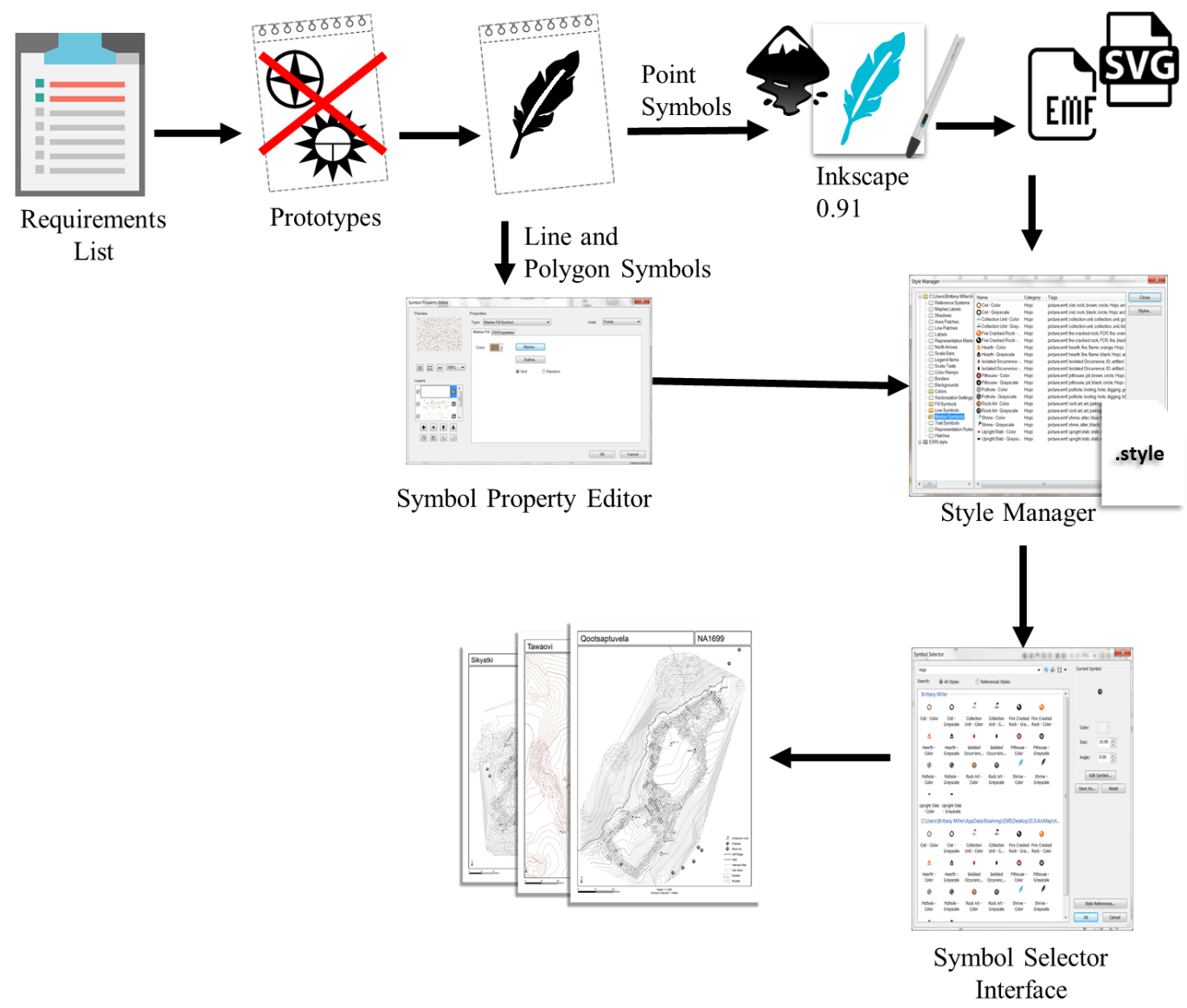

Figure 5-3: Workflow for Creating the Hopi Archaeology Set Symbology.

The client examined multiple prototypes of the symbol set until deciding upon a final symbology. Similar colors and shapes in the symbology set depict conceptual relationships between features. Excavation features (i.e. site boundaries, collection units, and potholes) use the same gray hue to denote the association between these recently created object. Similarly, fire cracked rock and hearths use the same red-orange hue to signify fire. Fire cracked rock and rock art use a hexagon to represent a rock feature. Figure 5-4 shows the ten symbol markers designed in Inkscape 0.91. 


\begin{tabular}{|l|c|c|c|}
\hline \multicolumn{1}{|c|}{ Feature } & Image & Color & Grayscale \\
\hline Cist & & & \\
\hline Upright Slab & & & \\
\hline Rock Art & & \\
\hline $\begin{array}{l}\text { Fire Cracked } \\
\text { Rock }\end{array}$ & & \\
\hline Hearth & & \\
\hline $\begin{array}{l}\text { Shrine } \\
\text { Unit } \\
\text { (looting) } \\
\text { (artifact) }\end{array}$ & & \\
\hline Pithouse & & \\
\hline
\end{tabular}

Figure 5-4: Marker Symbols in the Hopi Archaeology Set.

Spirals are a common artistic symbol that occurs in many Hopi petroglyphs. Due to its simple iconic shape, the spiral was incorporated into the rock art symbol. In addition, though there were many prototype versions to illustrate a shrine, the client determined that a feather symbol best represented the Hopi people. Since the color blue is often associated with wisdom and peace, a dark turquoise hue compliments the monochromatic warm oranges and brown hues used to represent an arid landscape.

The origin coordinates for the marker symbols are located in the center of the designs except for the collection unit and shrine symbols. Using the Symbol Property Editor, the adjusted symbols represent the exact coordinates more precisely by offsetting the symbol. The coordinate points are located at the base of the flag and tip of the feather. This ensures that the exact coordinate is not obscured by the symbol used to represent the point (Figure 5-5). 


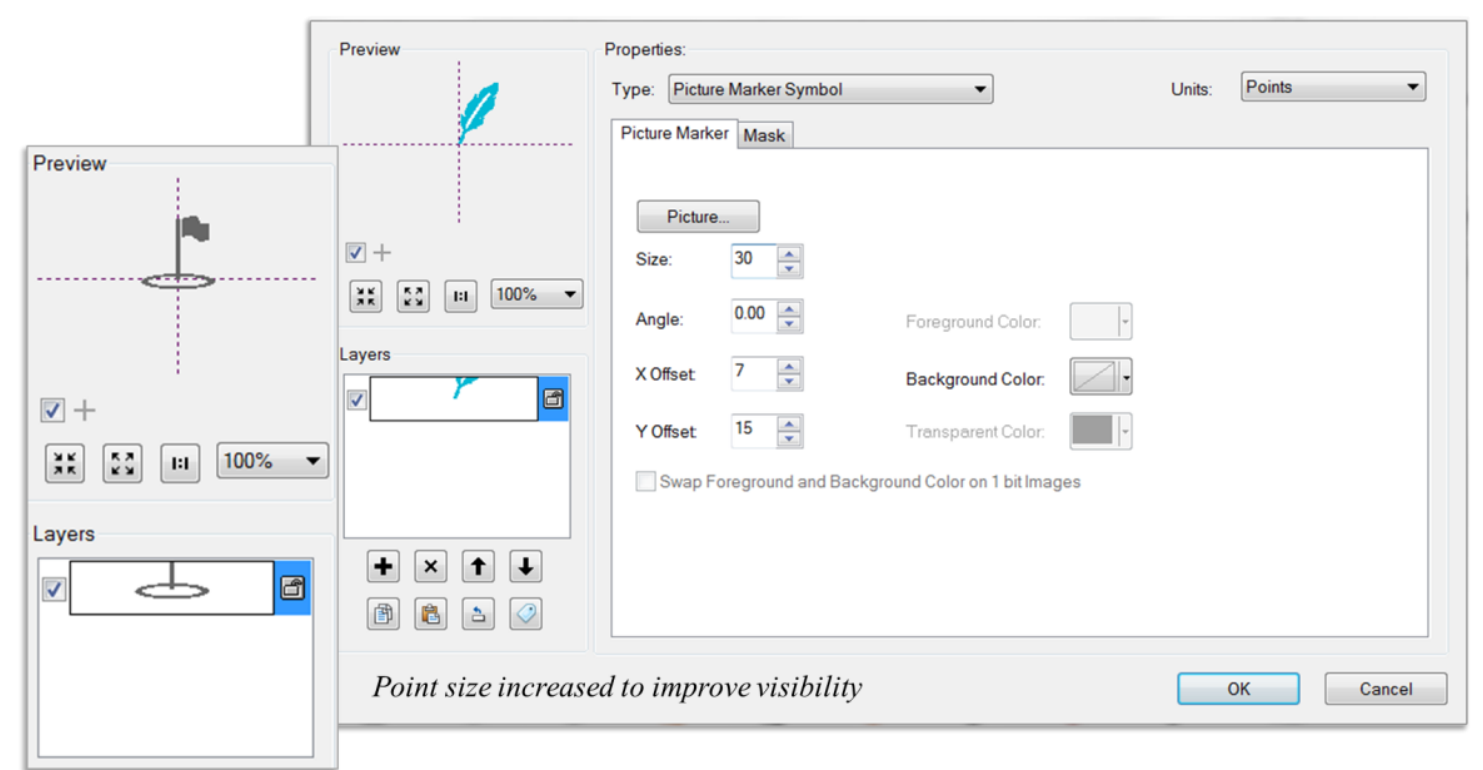

Figure 5-5: Adjusted Marker Symbols to Represent Point of Origin.

The fill patterns for rubble and midden used a random pattern generated within the Symbol Property Editor. Sharp-edged generic shapes, such as triangles and diamonds, simulate pottery shards found in midden sites, while rubble used a series of differentsized brown circles to represent scattered stones. Both of these fill patterns generated a similar stippled effect found in nineteenth century maps, but used additional shapes and colors to differentiate between the two archaeological features. The rock outcrop polygon fills used a picture pattern already installed in Esri's Symbology used to represent park trees, but the edited pattern color now represents a rocky area. The line patterns found in the reservoir and ash stain symbols were custom made in the Symbol Property Editor using the Template tab. Figure 5-6 contains the polygon fills included in the custom symbology set. 


\begin{tabular}{|c|c|c|c|}
\hline Feature & Image & Color & Grayscale \\
\hline Midden & & $\dot{\Delta} \Delta \diamond^{\Delta}$ & $\Delta \Delta \Delta \Delta^{\Delta}$ \\
\hline \multicolumn{4}{|l|}{ Rubble } \\
\hline \multicolumn{4}{|l|}{ Ash Stain } \\
\hline \multicolumn{4}{|l|}{ Reservoir } \\
\hline Rock Outcrop & & & \\
\hline (Bedrock) & & & \\
\hline
\end{tabular}

Figure 5-6: Polygon Fills in the Hopi Archaeology Set.

Designing the stone wall and inferred stone wall symbols was one of the more challenging aspects of the symbology set. Stone walls are observable rock foundations that indicate that a wall existed at an exact location; inferred walls are uncertain and theorized to exist due to patterns found within rubble surrounding an archaeological site. To mimic the stippling technique for both walls, four offset dashed marker lines shifted away from the origin line between -0.25 to 0.25 points. The Template tab in the Symbol Property Editor adjusted the lines to determine the distance intervals between each marker symbol (Figure 5-7). The challenge existed in maintaining the same distance and stippled effect at scales ranging from 1:500 to 1:2,500. At larger scales, such as 1:500, the lines became thinner and the markers decreased in size automatically. Maps with a scale of 1:1,000 or more rendered the stone wall and inferred wall symbols as intended, but when adjusted, 1:500 scale maps still maintain a scattered rock appearance. 


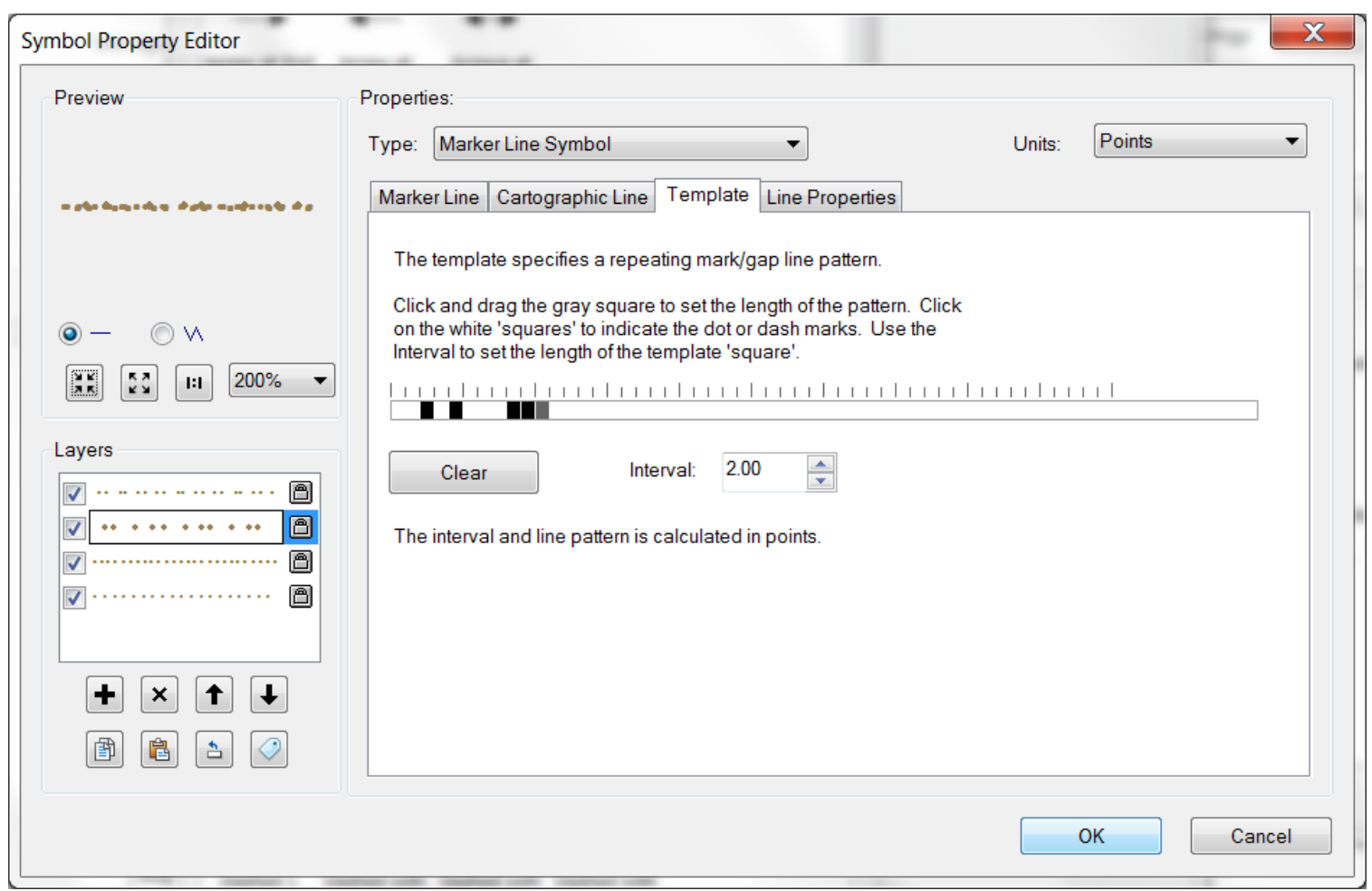

Figure 5-7: Symbol Property Editor.

A darker brown color denotes stone walls that are observable while the lighter hue represents walls inferred by archaeologists. A thin line was included in the stone wall symbol to help ground the linear pattern, but maintain the appearance of scattered stones. The inferred walls symbol more loosely separates the marker symbols to denote the uncertainty of the wall's existence. The inferred wall and rubble symbols were designed using the same light brown hue to show the correlation between the existing rubble and the notion that a stone wall may have been constructed in that location.

The archaeological site boundary was custom designed using the Template tab and continued the conceptual relationship between excavation features by being the same gray hue. Two additional line symbols were designed during the process: a contour line specifically for index contours and one for agricultural walls. This totaled the number of symbols in the set to 23 to represent 22 archaeological features. These additional symbols made the map designing process more convenient and accessible for the client when producing future maps. Figure 5-8 shows the line symbols contained in the Hopi Archaeological Set. 


\begin{tabular}{|l|c|c|c|}
\hline \multicolumn{1}{|c|}{ Feature } & Image & Color & Grayscale \\
\hline Site Boundary & & & \\
\hline Cliff Edge & & & \\
\hline Trail & & & \\
\hline Inferred Wall & & & \\
\hline Wall & & & \\
\hline Contour & & & \\
\hline Agricultural & & & \\
\hline
\end{tabular}

\section{Figure 5-8: Line Symbols in the Hopi Archaeology Set}

The symbols are in a .style format titled Hopi Archaeology Set and are available in the Style Manager in ArcMap 10.3. The client can import the symbology on his own desktop using the Style Manager and share the symbology set with other archaeologists at his own discretion. The client can also add, edit, or delete symbology within the file. The final style format also includes the colors used to produce the marker symbols, so the client can continue using the colors. Tags were included on the symbology and can be entered in the search bar of the Symbol Selector to query desired symbols quickly (Figure $5-9)$. 


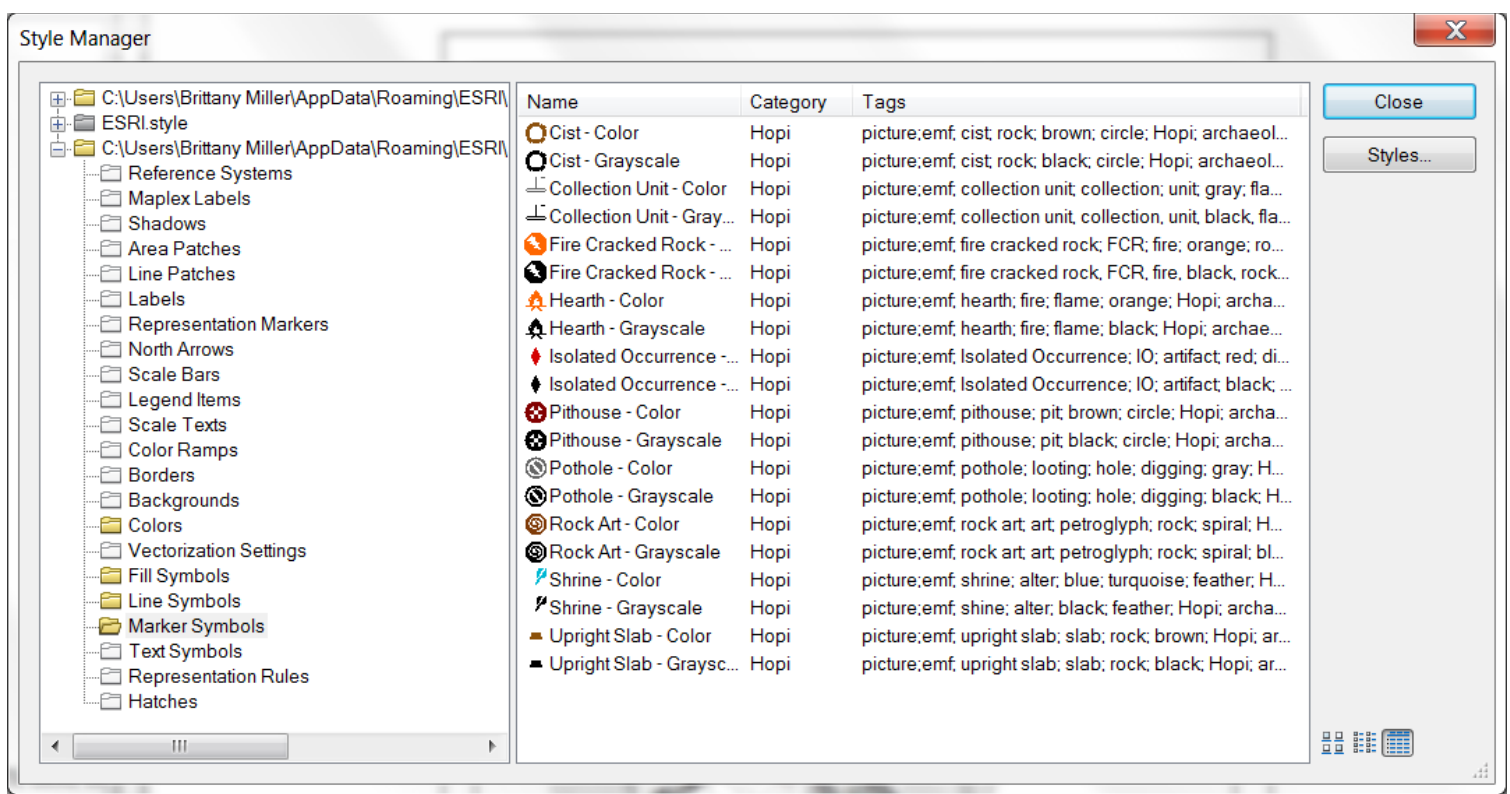

Figure 5-9: Hopi Archaeology Set within the Style Manager

The Symbol Selector automatically adds the symbology set from the Style Manager. The client can select any desired marker symbol and adjust the size to represent the map scale. For lines and polygons, the client can adjust the color and size using the Symbol Property Editor. Figure 5-10 shows the ArcMap10.3 Symbol Selector containing the returned Hopi Archaeology Set symbology after entering the universal tag Hopi into the search bar. 


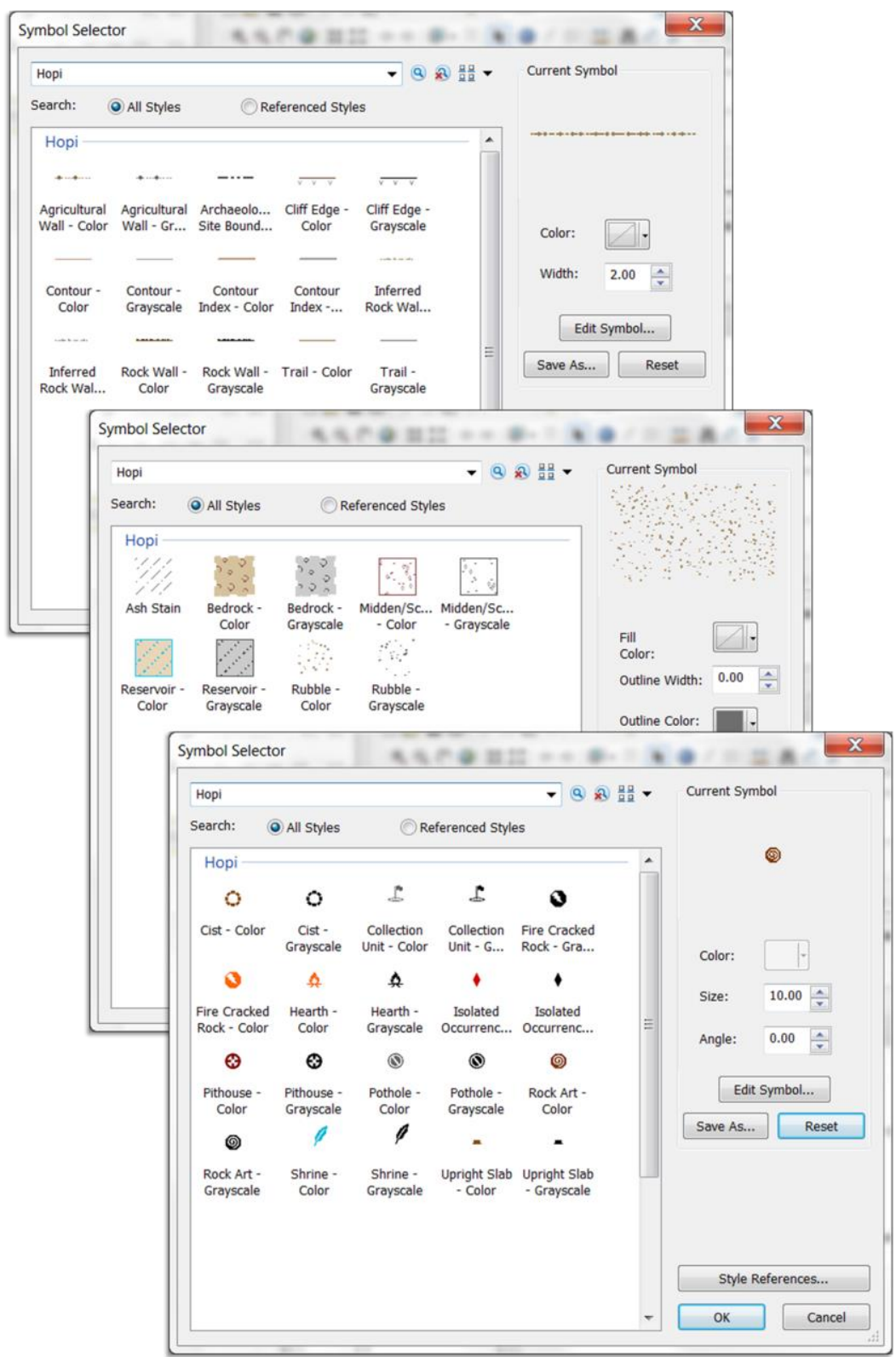

Figure 5-10: Symbol Selector Interface

\subsection{Symbology Survey}

The survey tested the intuitiveness of the new custom symbology against the formerly used default symbology. The survey was not a formal research study, but rather an anonymous review that provided feedback on the prior and custom symbol sets. Built through the online survey builder website, Survey Monkey, the survey contained two sections. The first section tested individual map variables against each other by having participants determine which of two maps was visually more appealing. The second portion of the survey asked participants to select what type of archaeological feature best matched one of two symbols. This portion tested the intuitiveness of the default 
symbology first, and then moved to the custom symbology set. Each symbol appeared twice against a different symbol from the same set.

University of Redlands undergraduate and graduate students currently enrolled or previously enrolled in archaeology, anthropology, history, environmental science, or GIS were eligible to participate. To administer the survey, the Collaborative Institutional Training Initiative (CITI) training for human subject testing was completed and consisted of 16 session modules. The Institutional Review Board (IRB) reviewed the application with the two attached CITI certificates. While IRB approved the survey, there were few options for administering the survey to the student population. After consulting with the Student Life department, students are not able to mass email the student population, and the department did not grant a waiver for this project. The contingency plan involved emailing professors teaching the specified disciplines with the expectation that they forwarded the email to students. Current and former cohorts from the University of Redlands MS GIS program also received emails. The survey opened on May 22, 2016 and closed June 9, 2016 with 29 participants. Chapter 6 reviews results and survey trends in further detail. Figure 5-11 provides page examples of the survey. 


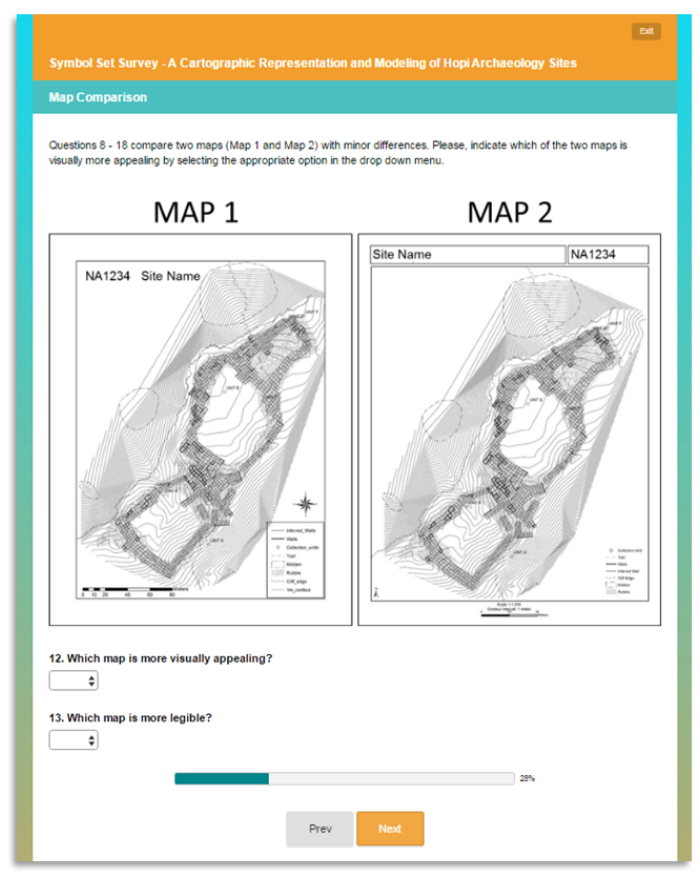

(a)

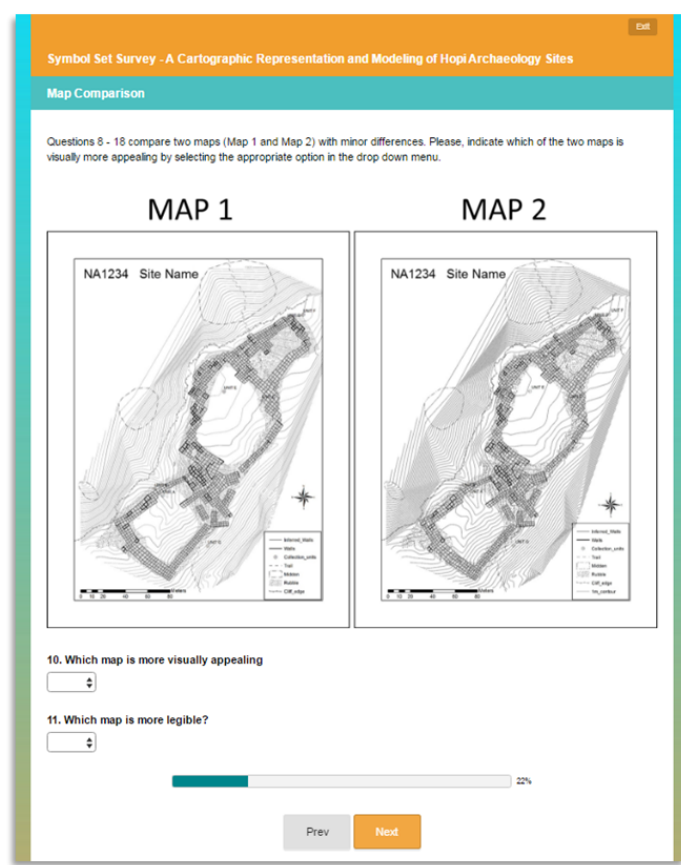

(b)

Section 1 compared map variables between two maps.

Here, the map layout (a) and contour lines (b) are compared.

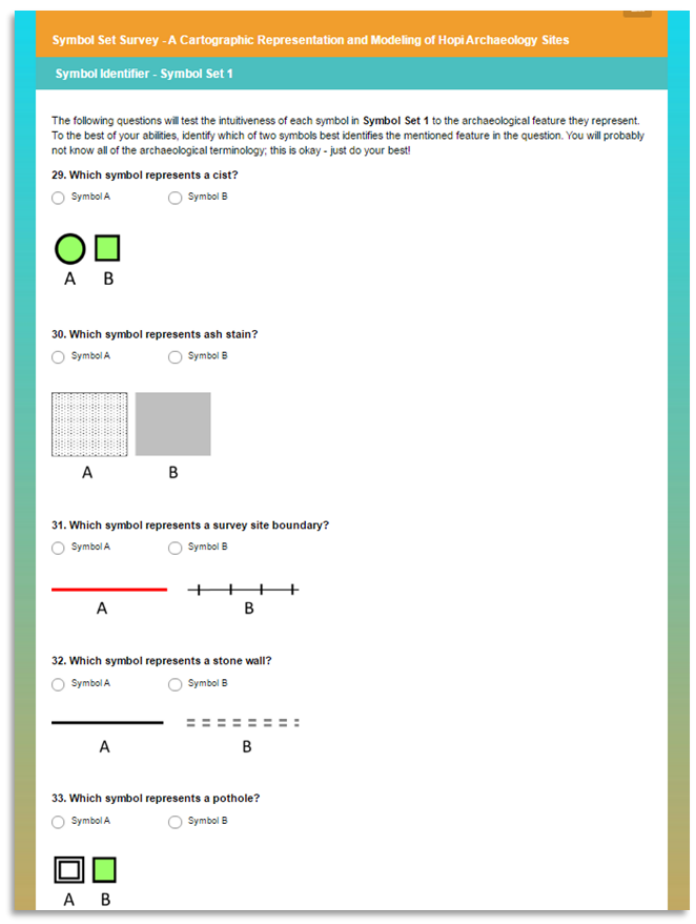

(c)

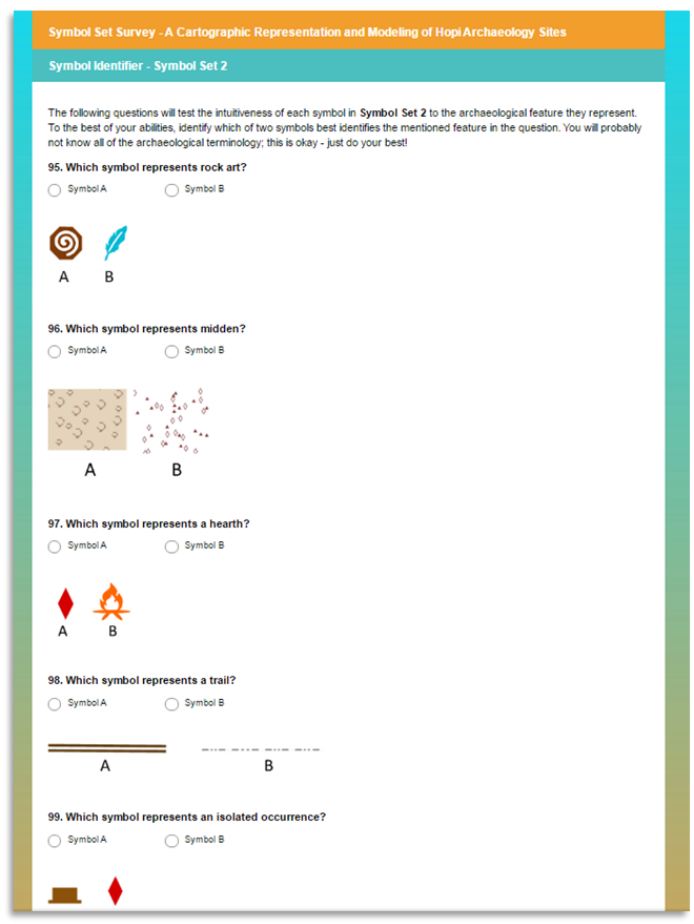

(d)

Section 2 compared the original and custom symbology.

The first portion tested the original symbols (c) while the second portion (d) tested the custom symbology.

Figure 5-11: Samples of the Survey 


\subsection{Map Series}

The map series contained 144 maps of the 48 archaeological sites. Since survey boundaries often contained several smaller archaeological sites, the surveys needed multiple maps to show each site at a specific scale. All of the maps scales range in 1:500 increments ranging from 1:500 to 1:2,500 at site level. Though some sites are small, the 1:500 scale was the set minimum to maintain consistency throughout the series. Each map included color and grayscale version for printing purposes and was exported as PDF files, which exported the layers individually. The client can select which layers are visible within the PDF to customize the document without editing and exporting a new map in ArcMap (Figure 5-12).

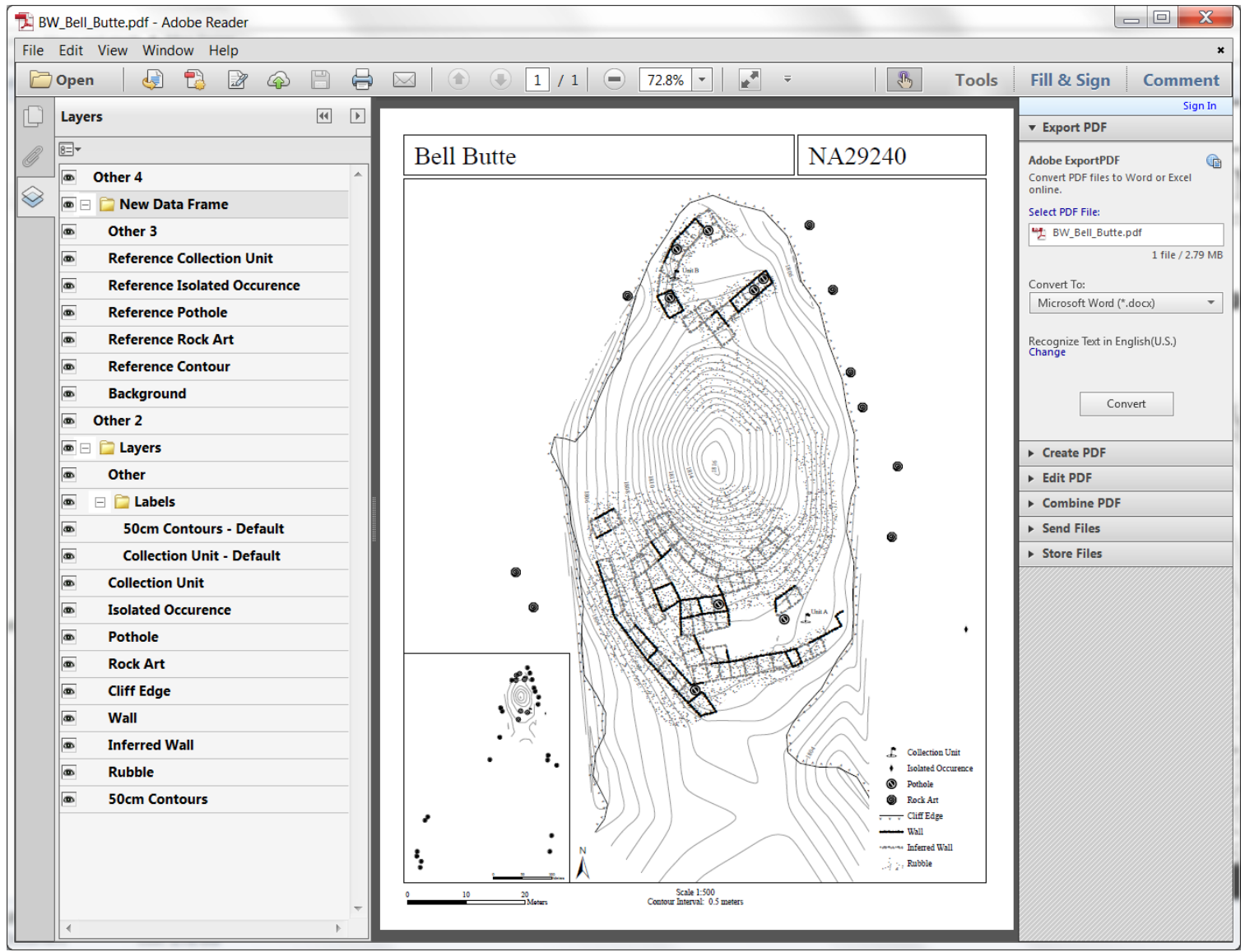

Figure 5-12: PDF Layers Tab

Figure 5-13 shows the Maplex labeling interface used to label the contours and collection units in the maps. The client provided the contour line shapefiles created from GPS points collecting elevation. Contour lines were oversimplified and the intervals varied 3 meters, 2 meters, 1 meter, 0.5 meters, 0.25 meters, 0.2 meters, and 0.1 meters. The inconsistent contour intervals were difficult to represent with one standard contour index, so index intervals were set at 5 meters when possible, but some sites required indexes at every 1 or 2 meters. The Structured Query Language (SQL) within Maplex set 
labels only on the index contours in the same hue as the contour with a white halo to increase legibility.

If an attribute field noted the name of the collection unit, points received a label identifying the title of each unit. Six observed naming schemas existed for the collection unit labels, so the description fields required editing to follow the "Unit A" format.

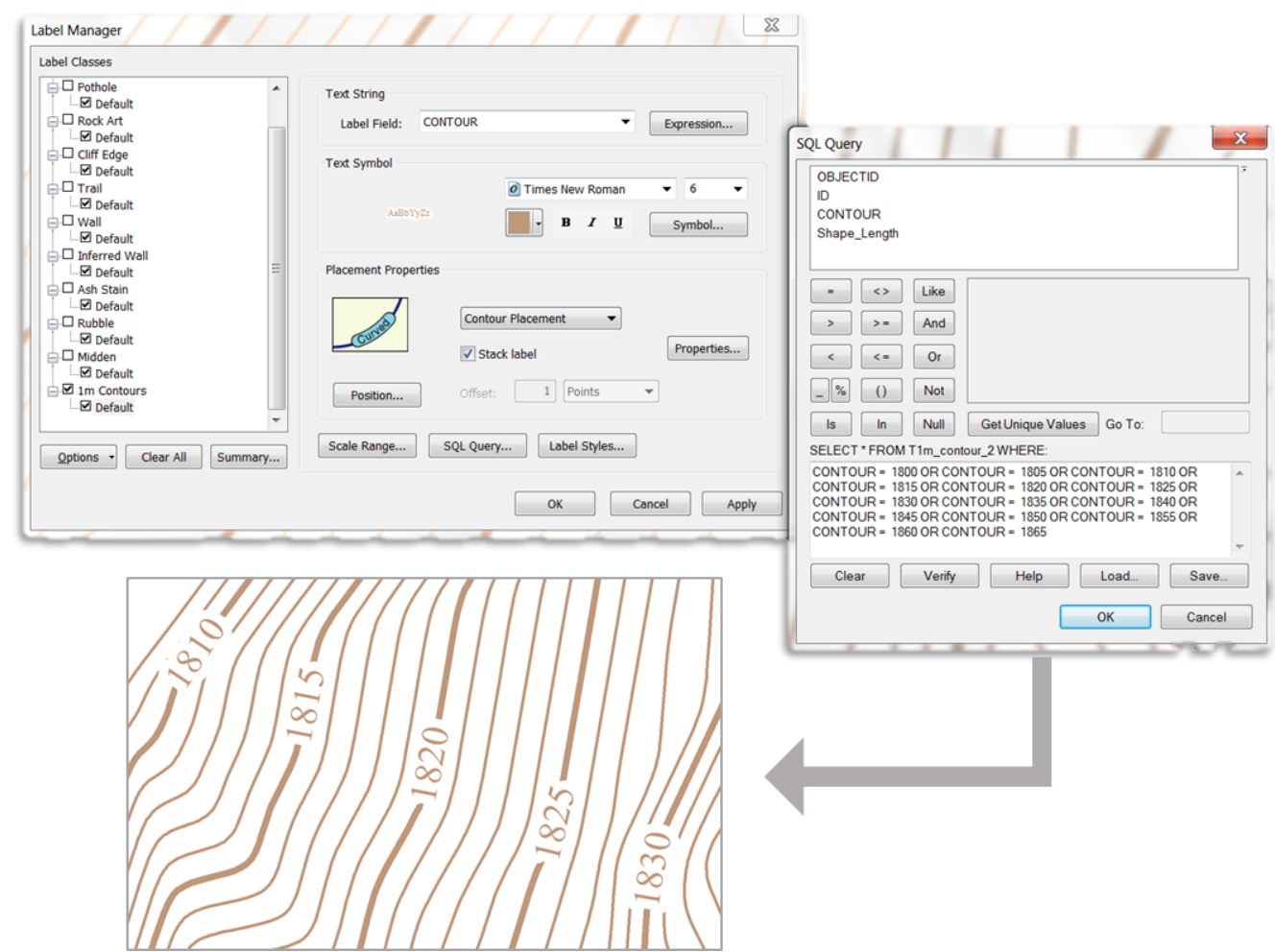

Figure 5-13: Maplex Labeling Interface

Figure 5-14 compares the traditional archaeological map on the left, the client's original design in the center, and the custom symbology applied on the right. The right and left images use the stippling technique to imitate the appearance of scattered stone. The traditional map uses heavier stippling to represent known wall alignments, and the lighter gray denotes inferred walls. However, the difference between the walls and inferred walls does not quickly communicate that information since the inferred walls appear more certain than the rubble. The client wanted the inferred walls to appear more uncertain than the original map by integrating the inferred walls within the rubble. The marker symbols used to create the inferred wall line were separated more than the markers used to represent a stone wall. Although the client wanted the inferred walls scattered further, separating and offsetting the markers even more dispersed the pattern too much and blended the marker symbols in with the rubble. 


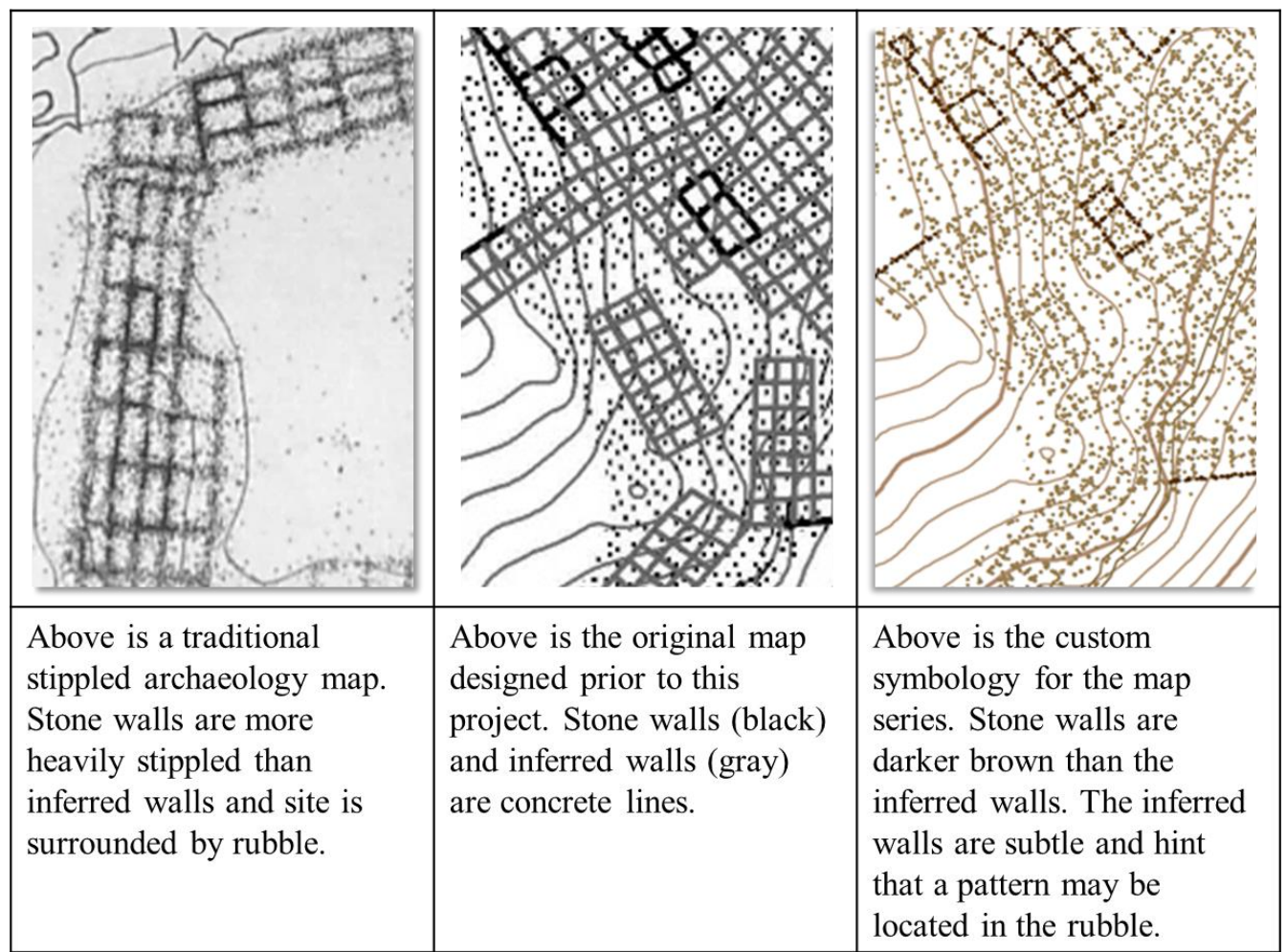

Figure 5-14: Comparison between Wall and Inferred Wall Symbology

Some of the sites contained feature classes for cliff edges. However, upon visual inspection, the custom symbology used to represent cliff edges did not always render properly automatically. This is because the symbol drew according to the direction the vertices were created. Sometimes the cliff edge symbology drew the triangular markers facing the correct direction of the steep elevation shift, while other times the triangles faced inward towards the archaeological site built on the mesa plateau. Therefore, the vertices on the feature classes for cliff edges required editing. The vertices were edited by opening the Sketch Properties interface during an editing session, selecting the desired feature, and then flipping the vertices so the cliff edge symbology render properly (Figure $5-15)$. 


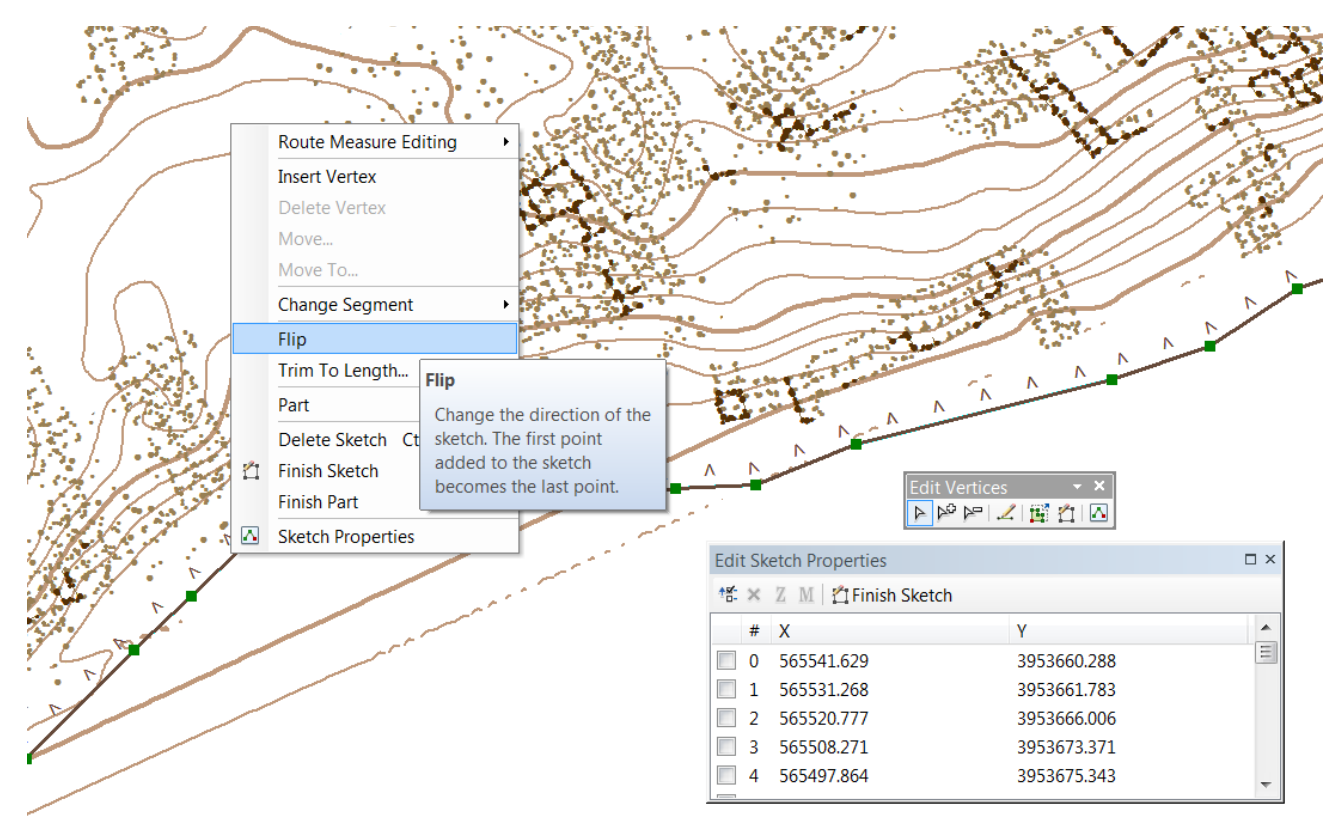

Figure 5-15: Sketch Properties to Flip Vertices on Cliff Edge Data

Archaeological feature classes appeared on some of the excavation sites not acknowledged on the required custom symbology list. Since no custom symbology existed to represent artifacts such as kivas, burial sites, and fences, the Caves font symbology housed in Esri's symbol selector represented these exceptions. One difficulty involved representing similar features that used different vectors. Point features represent cists in earlier records while lines portrayed cists in fieldwork that is more recent. Points used the custom symbology created to represent cists while the upright slab symbol generated a marker symbol line for cists portrayed as linear features.

\subsection{Three Dimensional Models}

Designing 3D models of the major sites was included in the original proposal, but the client provided the 3D input data later than expected in the development phase, reducing the time to create the models. The client delivered shapefiles for 18 sites, but it was determined that only $103 \mathrm{D}$ models could be produced within the given timeframe. The 3D data was imported into the existing geodatabase and transformed to the appropriate projection system and datum. The following requirements were determined for the $3 \mathrm{D}$ models:

- Room layers were extruded by 2.44 meters

- Models were placed in a more realistic landscape

- Models included archaeological features (i.e. midden, rubble, collection units, rock art, and potholes) using slightly modified default symbology

- Fly-though animations of the sites were created

The models were initially planned to be produced using ArcScene 10.3. However, the Digital Globe Foundation imagery did not overlay with the desired resolution over existing digital elevation models (DEM) and triangular irregular networks (TIN) rasters in ArcScene. After manually adjusting the resolution on the imagery using the Quality 
Enhancement for Raster Imagery tool, the pixels were still coarser than expected. Moreover, the DEMs and TINs generated by the collected GPS elevation points, though functional, did not represent the surrounding landscape. As seen in Figure 5-16, the imagery is clipped to the original TIN rather than showing the geography around the excavation site.

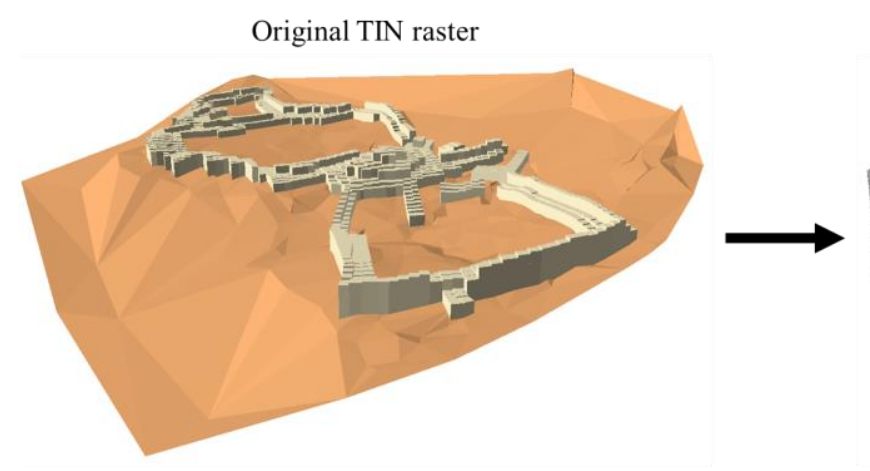

Figure 5-16: Digital Globe Imagery overlay in ArcScene 10.3

Since the prototypes did not provide the cartographic appeal desired from the 3D models, the project plan for rendering the models shifted from ArcScene10.3 to ArcGIS Pro 1.2. Using the Global View setting in ArcGIS Pro, the complete Digital Globe Foundation raster overlaid onto the pre-established elevation in Scene View and maintained its high resolution. Figure 5-17 shows how the high-resolution imagery rendered in ArcGIS Pro 1.2 and provided more insight on where structures existed in relation to the geography. Originally, the prototypes extruded the 3D layers using the Floors field to show the building height using the SQL statement "Floors * 2.44." However, after reviewing the models with the client, the data for the floor height are inaccurate and require updating. Although visually less interesting, all of the models universally extruded the 3D layers at 2.44 meters. 


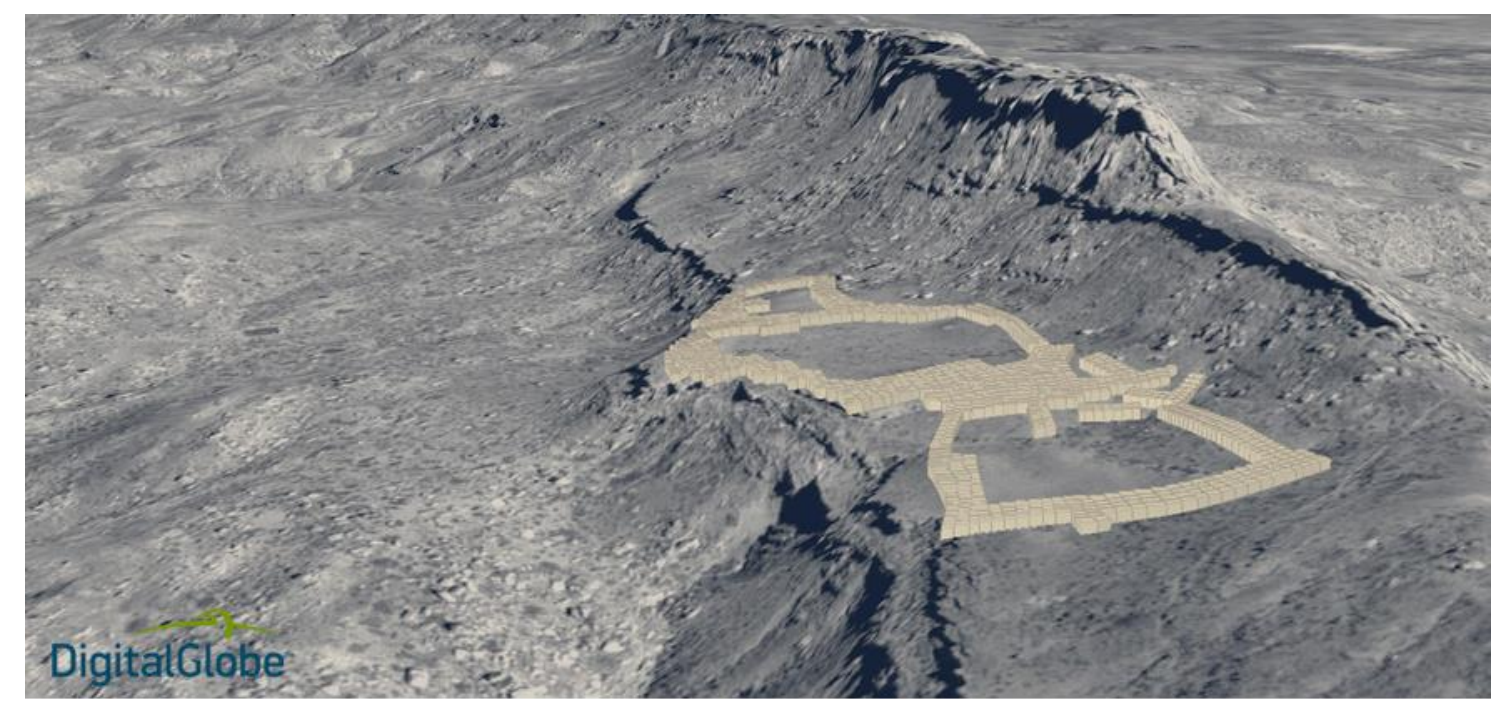

Figure 5-17: Digital Globe Imagery overlay in ArcGIS Pro 1.2

The 3D models also maintained a standard appearance similar to the print maps. Though incorporating the custom symbology into a 3D representation could produce interesting results, doing so was out of scope. Instead, a semi-custom symbology set was designed using default symbology in the same color schema as the 2D symbology. Transparent, solid-colored polygon fills using rose dust and tan hues represent midden and rubble; the gray pin icon and the cylinder identify collection units and potholes; a diamond shape in a dark brown-red signifies petroglyphs (Figure 5-18). These symbols were saved in a new .style format titled Hopi_3D and added to the Symbol Selector to produce the 3D models more efficiently. The client can use this symbology to create future models of archaeology sites and maintain a standardized appearance. The structures used Esri's default polygon fill for buildings. An atmospheric illumination set for 5:30:00pm Arizona time zone provided additional cartographic effect and shadowing to the models. 


\begin{tabular}{|l|c|c|c|}
\hline \multicolumn{1}{|c|}{ Feature } & Image & 3D Symbol & 2D Symbol \\
\hline Midden & & & \\
\hline Rubble & & & \\
\hline Collection & & & \\
Units & & & \\
\hline Rock Art & & & \\
\hline Potholes & & & \\
\hline
\end{tabular}

Figure 5-18: Symbology for 3D Models in Arc GIS Pro 1.2

Fly-through animations were also made of the 3D models. These videos let archaeologists look at structures and artifacts from an animated viewpoint rather than a static one to better understand how people lived in relation to the geography. The videos were exported as .mp4 files and are viewable using Windows Media Player. The client can decided to share these videos to the public during presentations.

\subsection{Summary}

The implementation phase consisted of developing multiple cartographic products. The first task organized the original data into a File Geodatabase to make the projection system consistent before moving to the artistic cartography aspect. The Batch Project geoprocessing tool standardized the projection system, and corrections to unknown spatial referenced data required manually edited. Visual checks on the data confirmed that the layers were spatially correct. This was performed using the Digital Globe Foundation imagery as a basemap to ensure that features matched rubble remains in the imagery when applicable and that the layers did not extend past the cliff edge. Input data were comprised of many shapefiles for similar features on one excavation site. To reduce data storage, input data were originally planned to be merge but still maintain their legacy through three added fields. Due to attribute inconsistencies in similar shapefiles, merging the data was out of scope; only the Mungyaovi survey data were merged since working with the numerous feature classes became unmanageable.

The next task was designing a custom symbology set for 21 archaeological features; the final set consists of symbology for 22 features. Point symbols were hand drawn in a third-party software while the other vector symbology used the ArcMap 10.3 Symbol Property Editor. The symbols stored within the style file titled Hopi Archaeological Set are accessible by uploading the file into the Style Manager. After 
completing the symbology, a map series used a similar aesthetics seen in nineteenth century maps to represent 48 archaeological sites. The map series totaled to 144 maps.

The maps were individually saved in PDF format with each layer exported separately so operational layers can be selected within the file.

The last portion of the workflow designed 3D models of ten major archaeological sites using ArcGIS Pro 1.2. Digital Globe Foundation imagery overlaid onto the topographical basemap in Scene View generated a more realistic landscape of the Hopi Reservation. A separate .style file for ArcGIS Pro contains a small symbology set for the 3D models. Fly-through animations portray the sites in a more realistic landscape and viewable in .mp4 format. 



\section{Chapter 6 - Results and Analysis}

This chapter addresses the product results and reports general observations on how the input data influenced the final project outcome. Section 6.1 discusses the overall result of the project and notes observations discovered throughout implementation. Section 6.2 provides an overview of the survey methodology and draws conclusions from the data results. This chapter also evaluates how well the map series communicates the information, what products are the most successful, and what areas in the project may not be developed to their full potential. By observing the results, the client can determine which products yield the most benefits and which aspects need elaboration.

\subsection{Product Assessment and Observations}

Comparisons between the symbology and map layouts in the two designs determined the effectiveness of the new maps to the original maps. Many of the cartographic elements in the original maps were inconsistent and overtly ornate. As Cynthia Brewer (2005) noted, "They [decorative elements] compete for the reader's attention and distract for the main message of the map" (p. 16). Brewer (2005) provided a specific example by observing the various direction indicators, specifically the north arrow, in Esri software. She discussed that north arrows are distinctive map elements but should not be so large or ornate that viewers mistake them as important map features (Brewer, 2005). The original maps used oversized and elaborate north arrows and scale bars. These elements diverted attention from the archaeological data presented in the map. The map series created during this project uses a simple north arrow and scale bar, so they are less distracting. The scale bars only consists of two divisions and are set at 50 meters or 20 meters depending on the map scale for easier legibility.

The map elements in the original maps were also inconsistent. For example, titles were located in the top-center or top-left, scale bar divisions ranged, and legend layers were in inconsistent order. The legends in prior survey site maps contained all of the symbology for the survey even though a particular map for a site boundary may only contain a few features. In the final map series, the title and archaeological catalog number are located in designated boxed sections on top of the map layout. To make the legends uniform, a master list was made that organized the archaeological features based on vector type and was referenced when arranging the layers in the map legend. Point data were alphabetized, and polygons were arranged depending on their visually heaviness. For example, the legend placed collection units above potholes and rubble above midden. Figure 6-1 offers an example of one original layout compared to the map layout used in the final map series. 

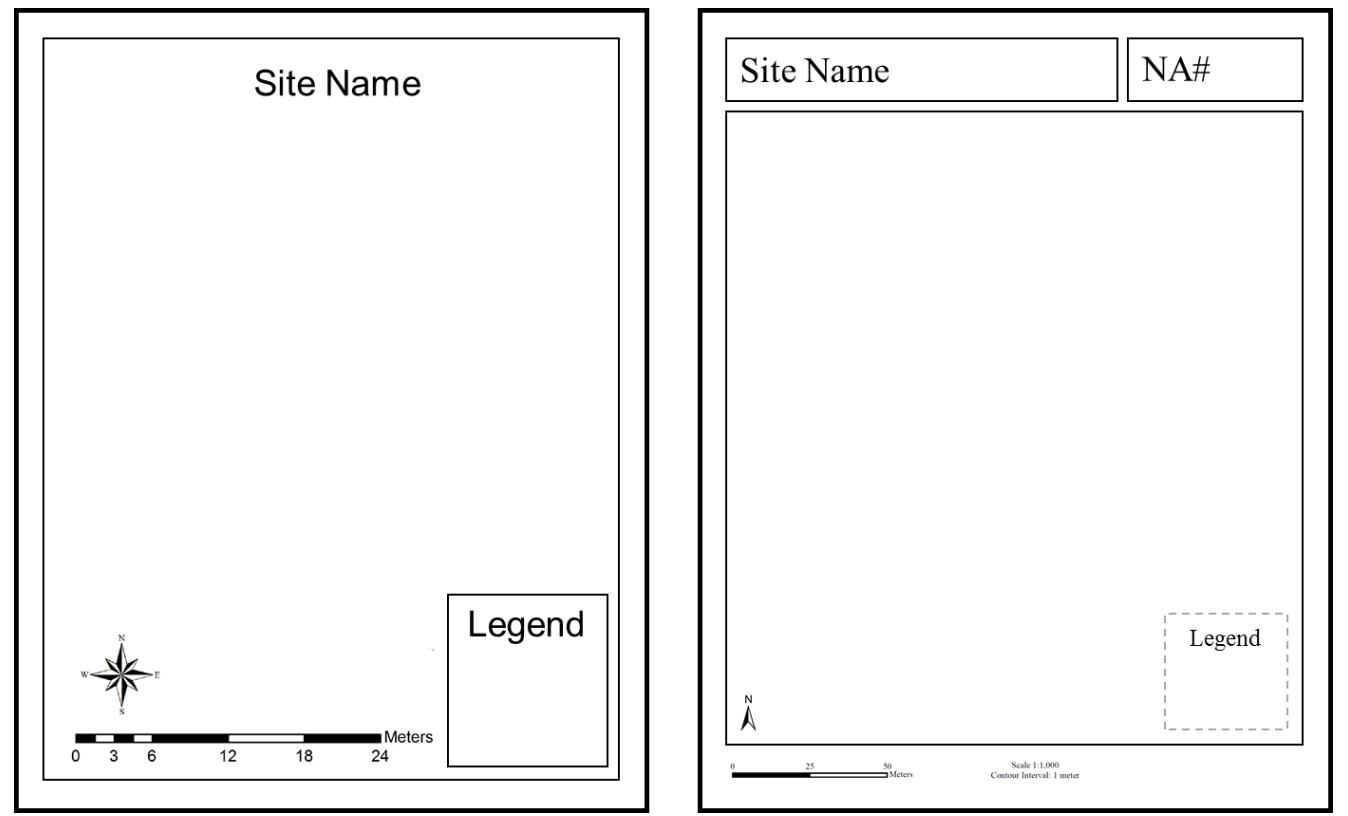

Figure 6-1: Original Map Layout (left) and Final Map Layout (right) Examples

The size of sites varied significantly, but having a consistent scale range was necessary for producing a cartographic standard. All scales increased at 1:500 increments that ranged from 1:500 to 1:2,500. An inlay map was also included in the map series for survey sites unlike the original maps. The inlays help specify where the large-scale boundary sites are located in relation to the other sites in the survey area. The inlay maps are also useful for showing artifacts located outside of the main archaeological site, such as rock art and potholes.

The point symbology adjusted on all scales and drew properly at 10-point size as determined in the symbol requirements. The stone wall and inferred wall symbology worked as intended at or over 1:1,000 scale. However, the lines automatically became thinner and the marker symbols became smaller at 1:500 scale. Although not solved perfectly to meet the ideal outcome, the symbols still adhered to the design requirements. During testing, the marker lines used to make the stone wall and inferred wall symbols were offset to a maximum of -1 to 1 point. However, the line adjustments blended the markers into the rubble. As Arthur Robinson (1995) noted, if point symbols making up a line become so dispersed, readers may mistake the points as multiple objects rather than denoting a linear feature. Setting the Reference Scale within ArcMap also did not provide the desired effect. Using the Reference Scale, maps at 1:500 maintained the symbology size for maps at 1:1,000 scale. While this generated the desired outcome for the walls, all of the other symbology and labels drew larger since they adhered to the same scale reference. Instead, the size of the markers in the stone wall and inferred wall symbology for maps at 1:500 scale were manually increased to maintain the same scattered appearance found on maps at 1:1,000 scale or higher. Figure 6-2 is an example of how the wall symbology rendered at different scales. 


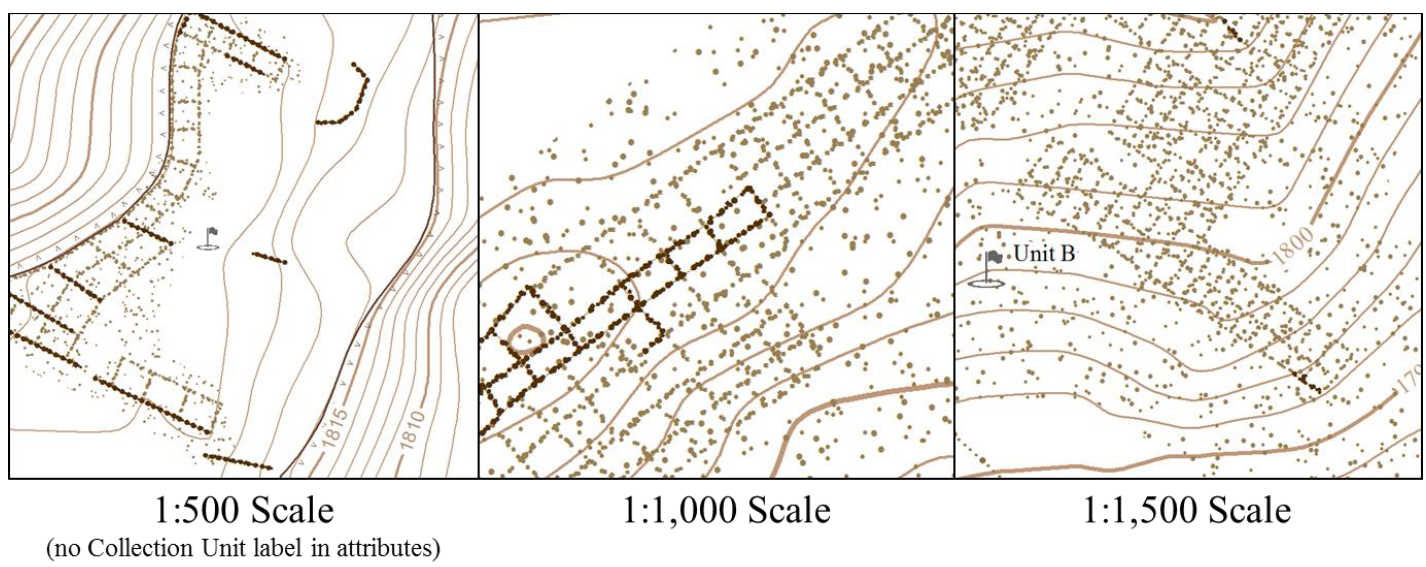

Figure 6-2: Wall and Inferred Wall Symbology at Different Scales.

Each symbol in the Hopi Archaeological Set possesses a default size and marker spacing assigned in the Style Manager. The specified defaults are the common sizes and spaces applied to the majority of features on the map series, but some symbols required alterations during the map design process. For example, large or small polygons representing midden needed spacing adjustments so the markers did not appear too dense or too spare. The scale of the map also affected how the polygon fills drew on the map, and manual adjustments were sometimes required.

One limitation in the map series is the shortage of data for some sites to produce a visually interesting map. A survey boundary consisted of several site boundaries that contained only a few recorded artifacts and did not provide a detailed depiction of the surrounding geography. For instance, one map portrayed an artifact and a trail found in a site boundary with no contour lines to depict the geography. Regardless, the symbology and layout designs remained consistent, and the client can add data to the existing maps as archaeologists continue to discover artifacts.

Ideally, one map would represent site survey data, but site boundaries and artifacts were so minute that creating a small-scale map of the entire survey area render inappropriately. Instead, a series of large-scale maps depicted a few site boundaries and included an inlay map showing the entire survey area. While this method depicts the minute artifacts found within the sites, the overall appearance of the maps are not visually exciting. Maps representing archaeological sites with more data are more engaging and provide a more detailed depiction of the area. Figure 6-3 is the grayscale version map representing three site boundaries in the First Mesa Survey site. The map presents information in a standardized format and includes an inlay map showing the relationship between the site boundaries on the survey. 


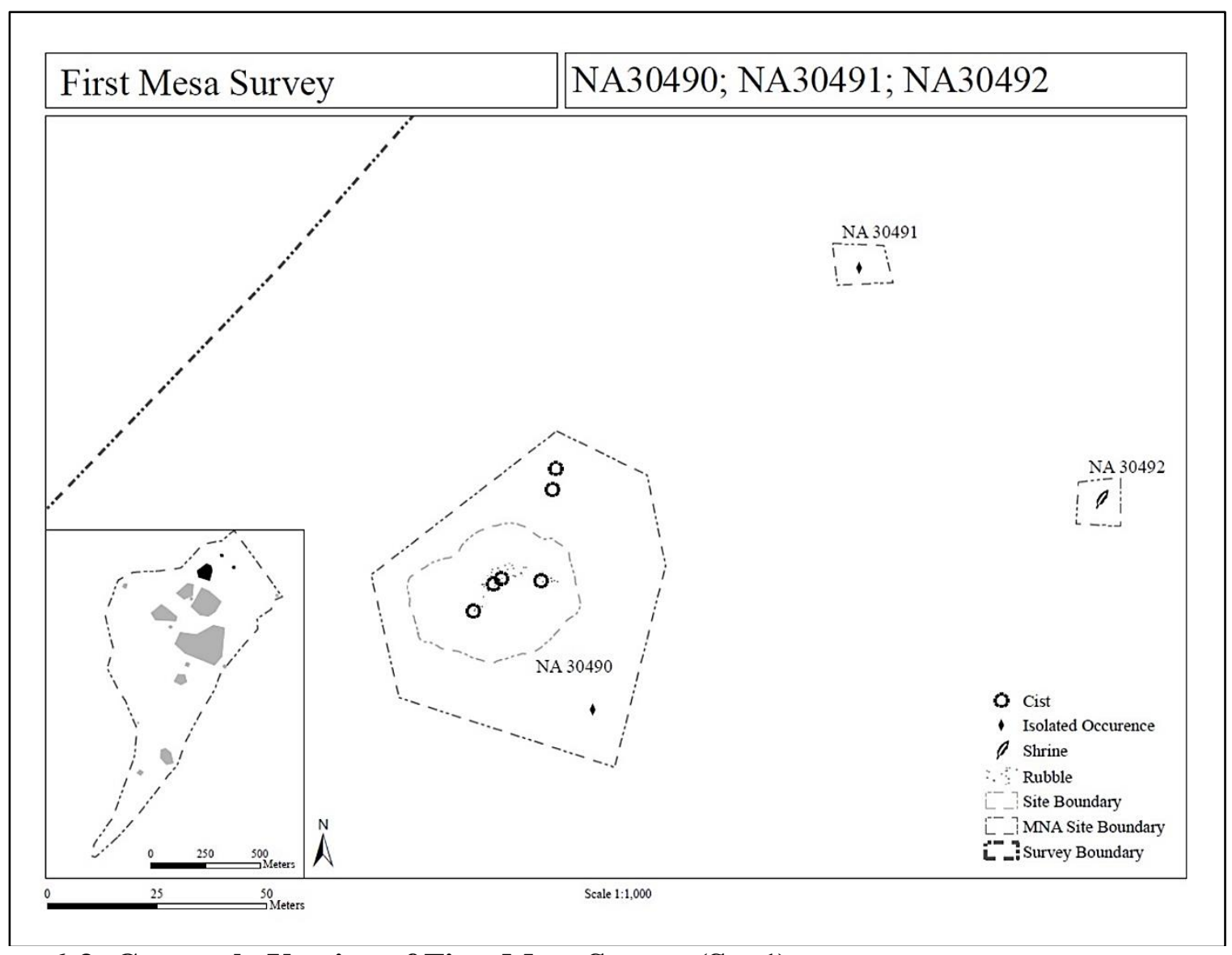

Figure 6-3: Grayscale Version of First Mesa Survey (Set 1)

The inconsistent contours were also a design limitation since intervals ranged from 0.1 to 5 meters. Archaeological structures exist on relatively flat areas, but where the sites are located vary from flat plains to mesa plateaus. The inconsistent intervals are the result of data collection methods that optimized the level of detail needed to represent elevation shifts. Generally, contour lines for larger sites and sites found on steeper terrain possessed larger intervals of 1 to 5 meters, while contour intervals for smaller sites or sites on flatter landscapes were less than one meter. The contour lines were originally represented using one symbol width without labeling the elevations, reducing the legibility and practicality of the map. The custom map series represents the contours using thicker labeled indexes and thinner interval lines. The index intervals ranged from 1 to 5 meters due to prior collection methods that maintained the topology detail for each site.

The map series and symbology set components of this project provided the client with a product for representing archaeological sites on the Hopi Reservation. The symbology set uses a monochromatic warm color scheme to portray the arid Arizona landscape. The symbology effectively represented Hopi culture by designing the symbols around the images provided by the client in the requirements list. Generating a scattered pattern for the stone walls successfully mimicked the stippling technique used in traditional archaeological maps. The legibility of the data was also improved in the series though a new map layout, labeling the collection units, and by labeling the index 
contours. Figure 6-4 is an example of the original map design created prior to this project. Though functional, some design elements limit effective communication.

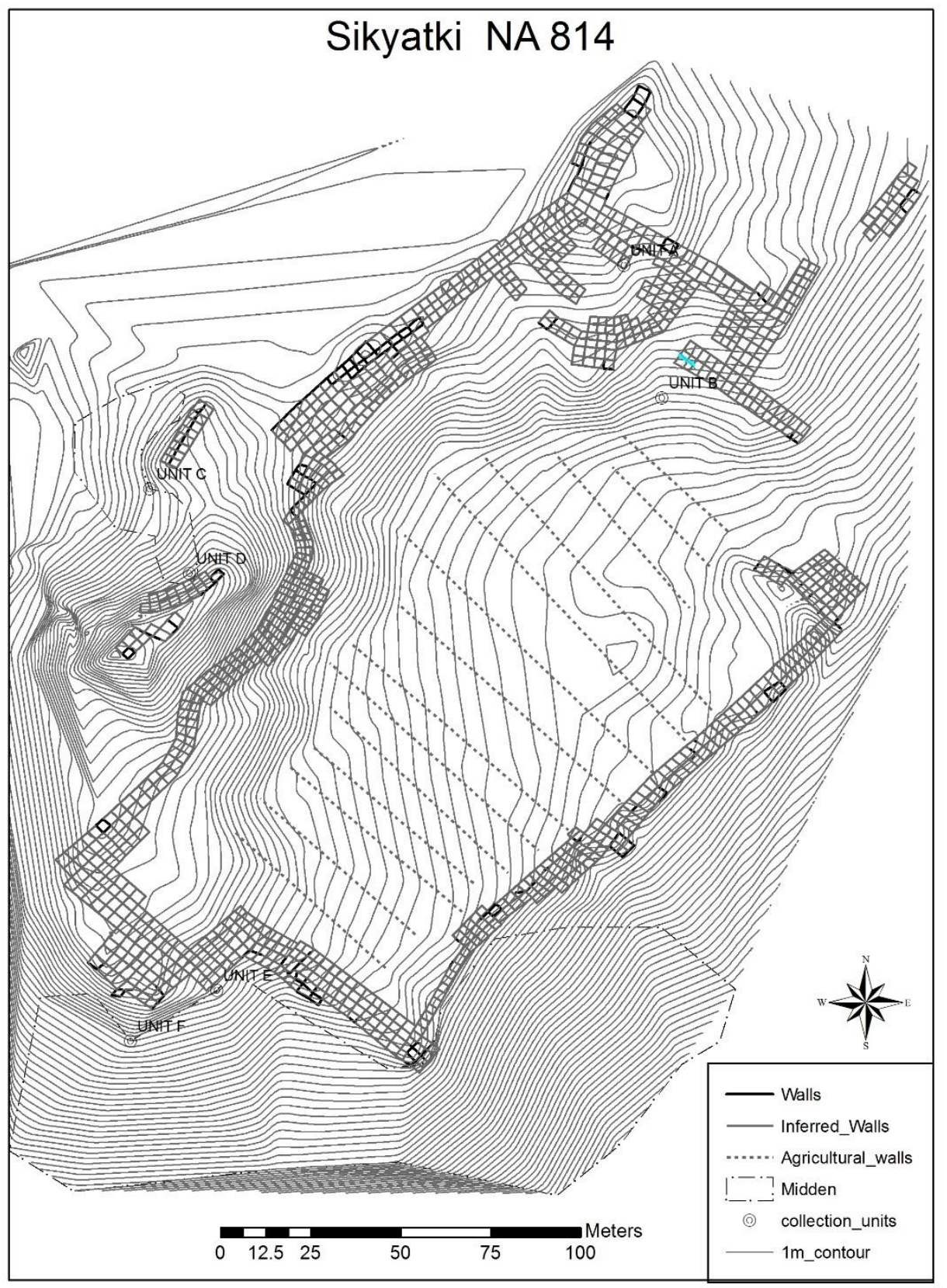

Figure 6-4: Prior Version of Sikyatki 
Figure 6-5 and Figure 6-6 illustrate the color and grayscale versions in the final map series for the Sikyatki archaeological site.

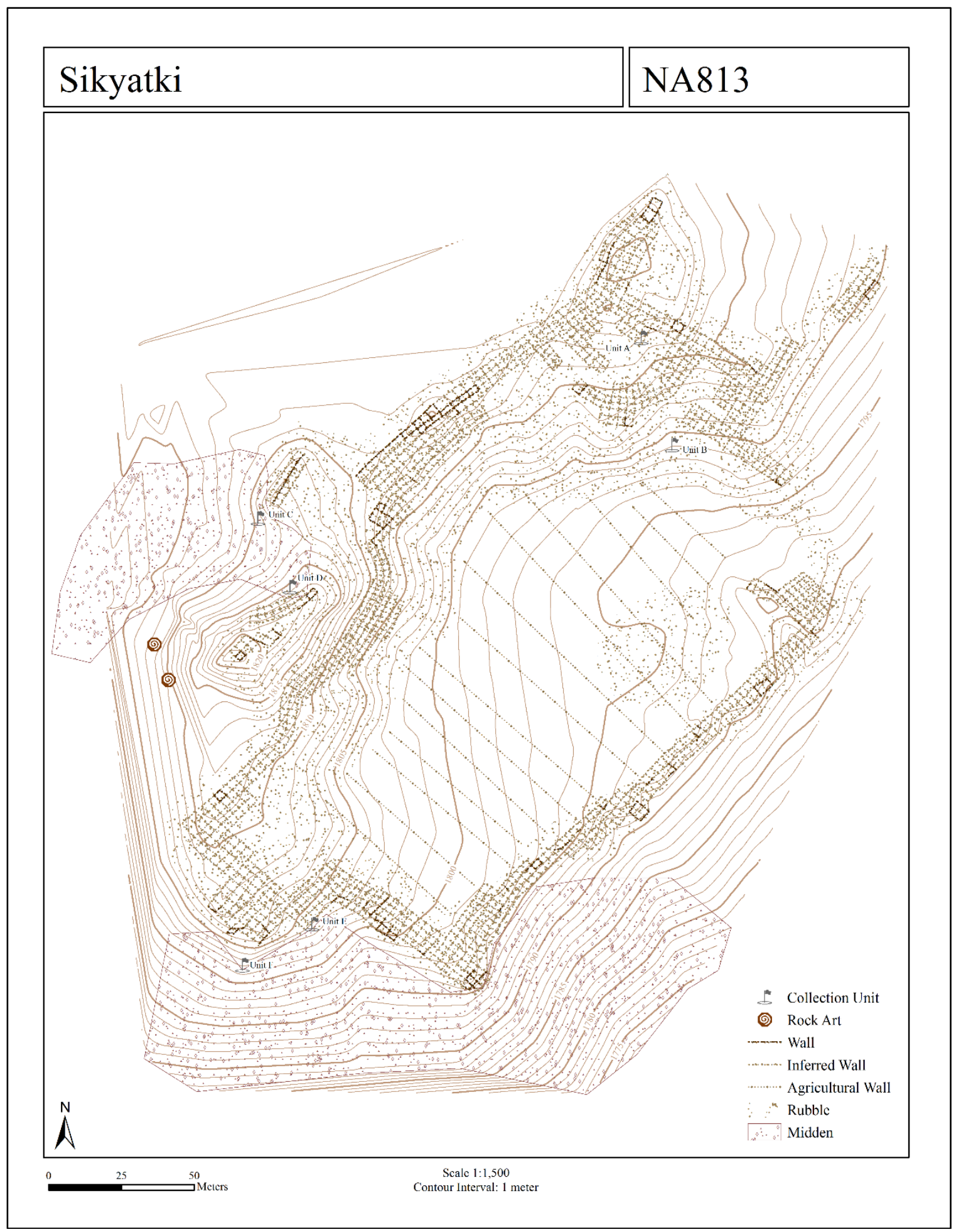

Figure 6-5: Color Version of Sikyatki 


\begin{tabular}{|l|l|l|l|l|}
\hline Sikyatki & NA813 \\
\hline
\end{tabular}

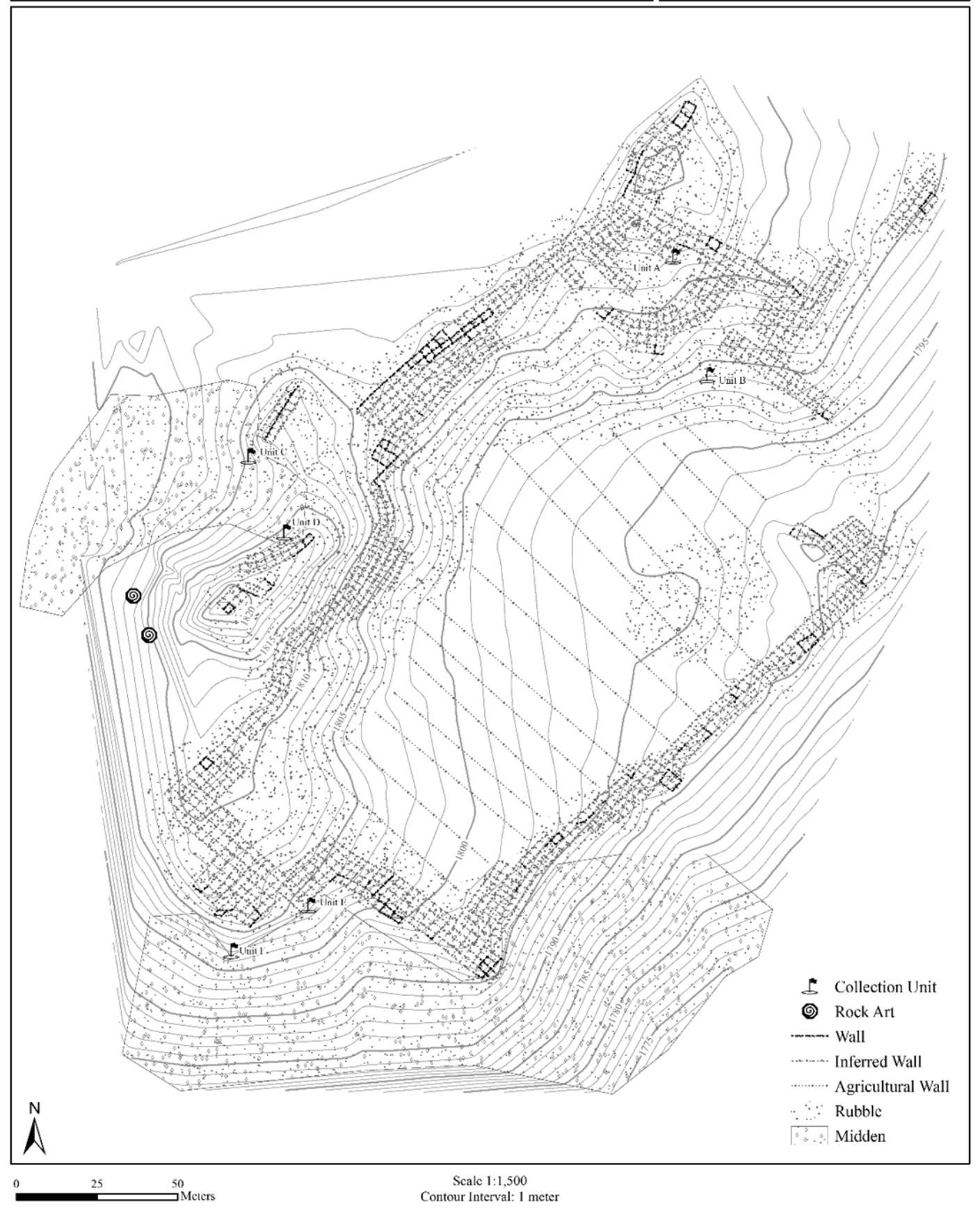

Figure 6-6: Grayscale Version of Sikyatki

The 3D aspect of this project never developed fully as expected in the proposal. This was in part due to the additional time spent on data scrubbing and organization, and the late arrival of the 3D data. Nevertheless, the 3D models satisfied the requirements given by the client, and the client was pleased with how the imagery from the Digital Globe 
Foundation rendered in ArcGIS Pro 1.2. Extruded by 2.44 meters, the 3D layers set the site in a more realistic environment with semi-custom symbology representing archaeological features. The fly-through videos were successfully exported and are visible through the Windows Media Player. Figure 6-7 and Figure 6-8 are examples of two of the ten 3D model created.

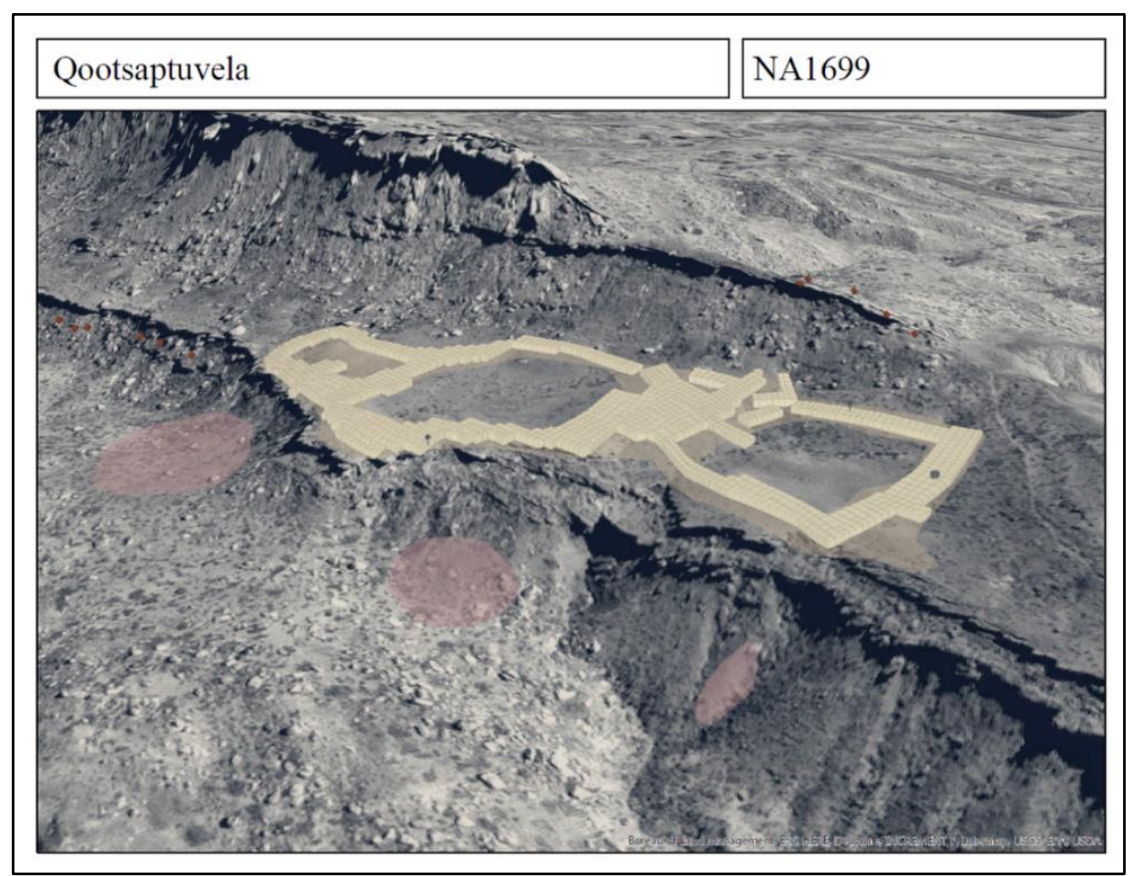

Figure 6-7: 3D Model of Qootsaptvela

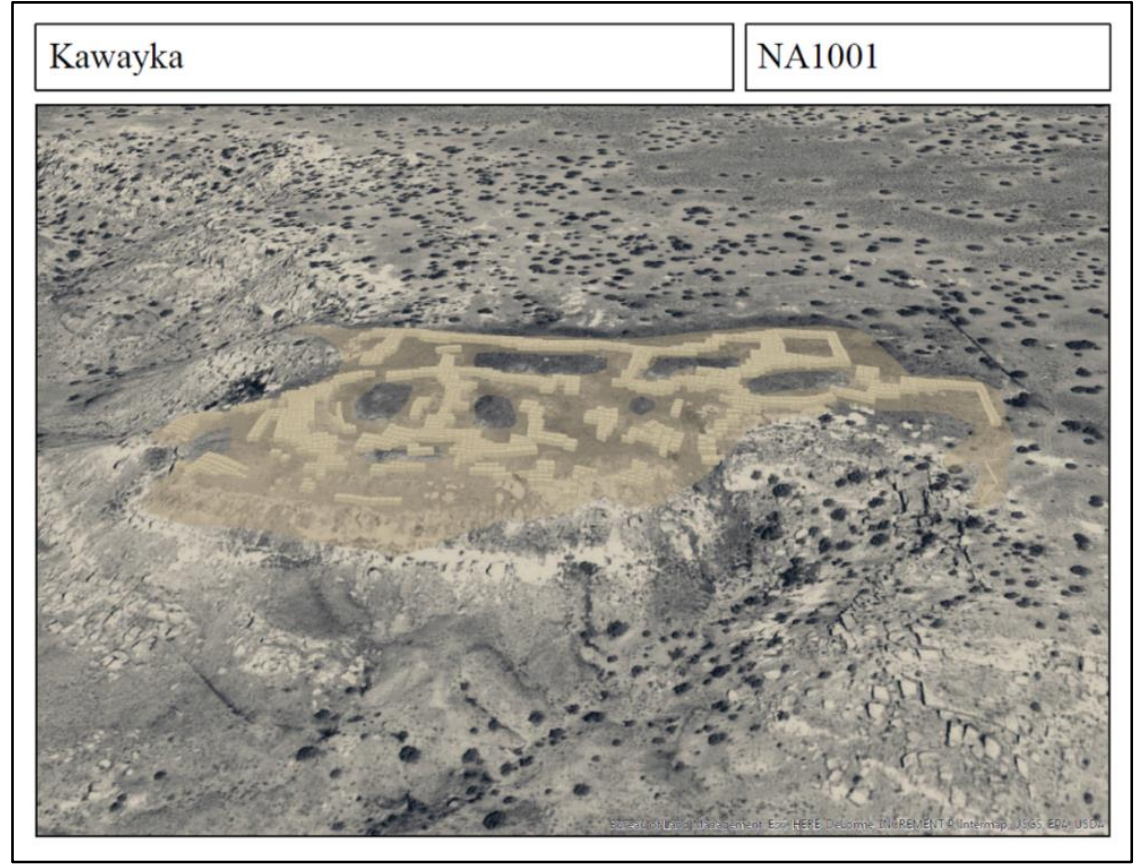

Figure 6-8: 3D Model of Kawayka 


\subsection{Survey Results}

Created through Survey Monkey, a link anonymously sent participates directly to the survey consent page. The survey opened on May 22, 2016 and closed June 9, 2016. A total of 29 subjects participated in the survey; 16 completed the survey, 12 finished the survey with incompletes, and 1 resigned. The survey contained 109 questions that asked participates to self-reflect on their map skillsets, compare the original and new map designs, and to match prior and custom symbology to the archaeological objects they represent. The first section asked participates to determine which of two maps was visually more appealing to test five individual map elements. The later section tested the intuitiveness of the original symbology set (Set 1) and then the custom symbology (Set 2) by asking them to identify which of two symbols matched a specific archaeological object.

Though undergraduate and graduate students enrolling or majoring in anthropology, archaeology, environmental science, history, or GIS were eligible to participate, graduate students or previous graduates majoring in GIS made up $83 \%$ of the participants $(0.03 \%$ were juniors and $16.97 \%$ left the question unanswered). When asked about their map reading and interpretation skills, all participates identified as average or above average. Most assessed their artistic skills between Average (26.09\%) or Above Average (39.13\%).

Most participants preferred Set 2 with the custom symbology when asked which symbology set was more cohesive and how sure they were about their answers. The overall results show that they received a greater percentage of the expected answers for Set 2 over Set 1 . However, many preferred the prior map designs that used the solid line symbols for the walls in the comparison section of the survey. Participates selected the expected answers for the contour lines and the map layout variables. The observed answers may have resulted by how the symbols appeared in the comparison section of the survey. One prior map incorporated the custom wall symbology while being compared to another prior map design with the original wall symbology. The custom wall symbology may not have appeared aesthetically against the other prior symbols on the map. The last question in the first section tested all of the variables by comparing the original map design (Map 1) to the new map design (Map 2). Participates said that Map 2 was more visually appealing at $60 \%$ and more legible at $57.89 \%$.

Figure 6-9 compares how participates assessed the symbology sets and their abilities to interpret the symbols after completing the Set 1 and Set 2 matching sections. Overall, they were surer of their answers for Set 2 and thought the custom symbology was more cohesive and easier to interpret. 


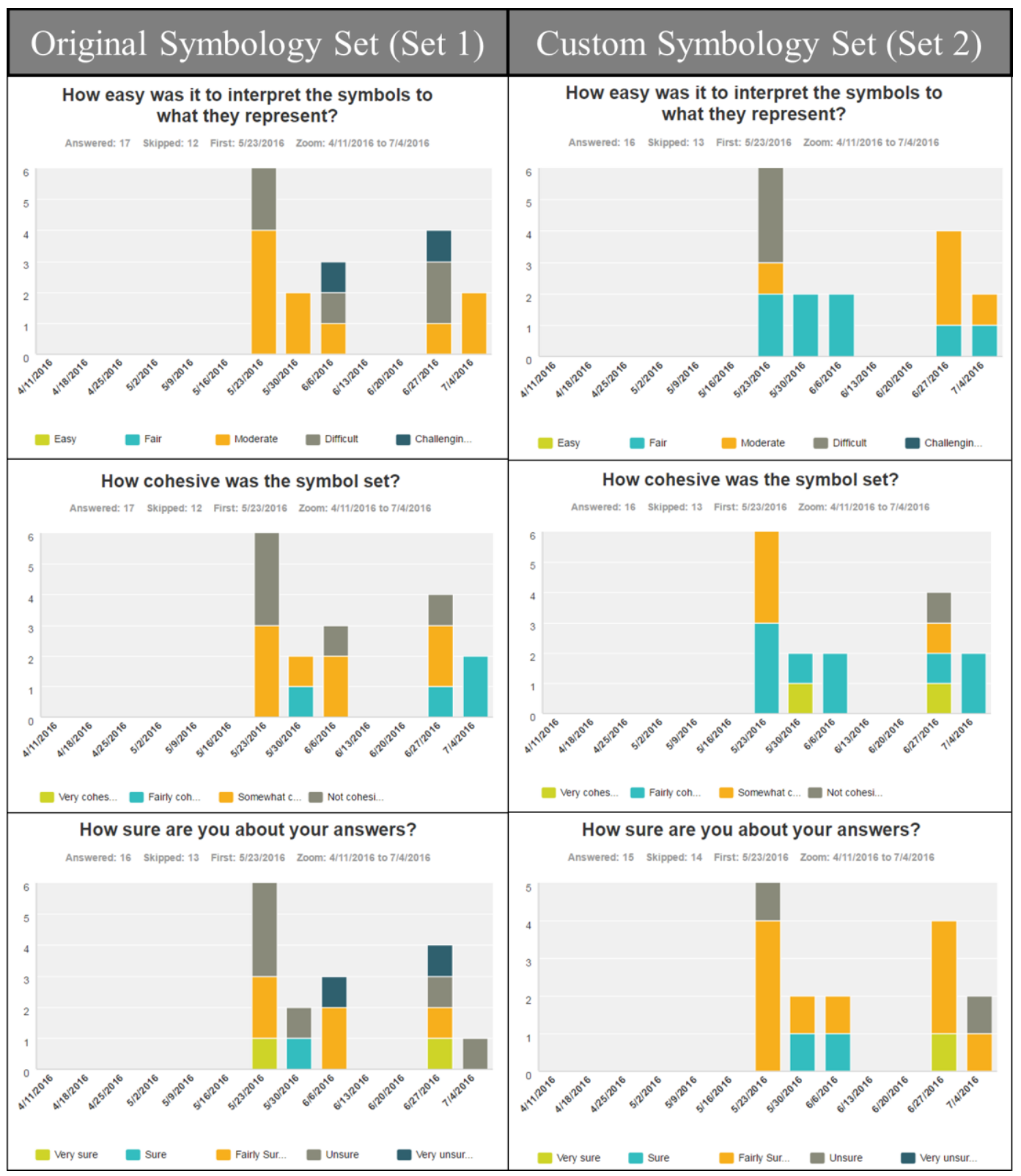

Figure 6-9: Survey Responses to Original and Custom Symbology Sets

Their performance and responses to the symbology correlate with the amount of expected answers they selected in both matching sections. Participants chose more of the expected answers in Set 2 over Set 1 by 22.8\%, indicating that the custom symbols are more intuitive even if the subjects were unfamiliar with the archaeological features being represented. The mean of expected answers for Set 1 was approximately $62 \%$ while the mean for Set 2 was approximately 84\% (Figure 6-10). 


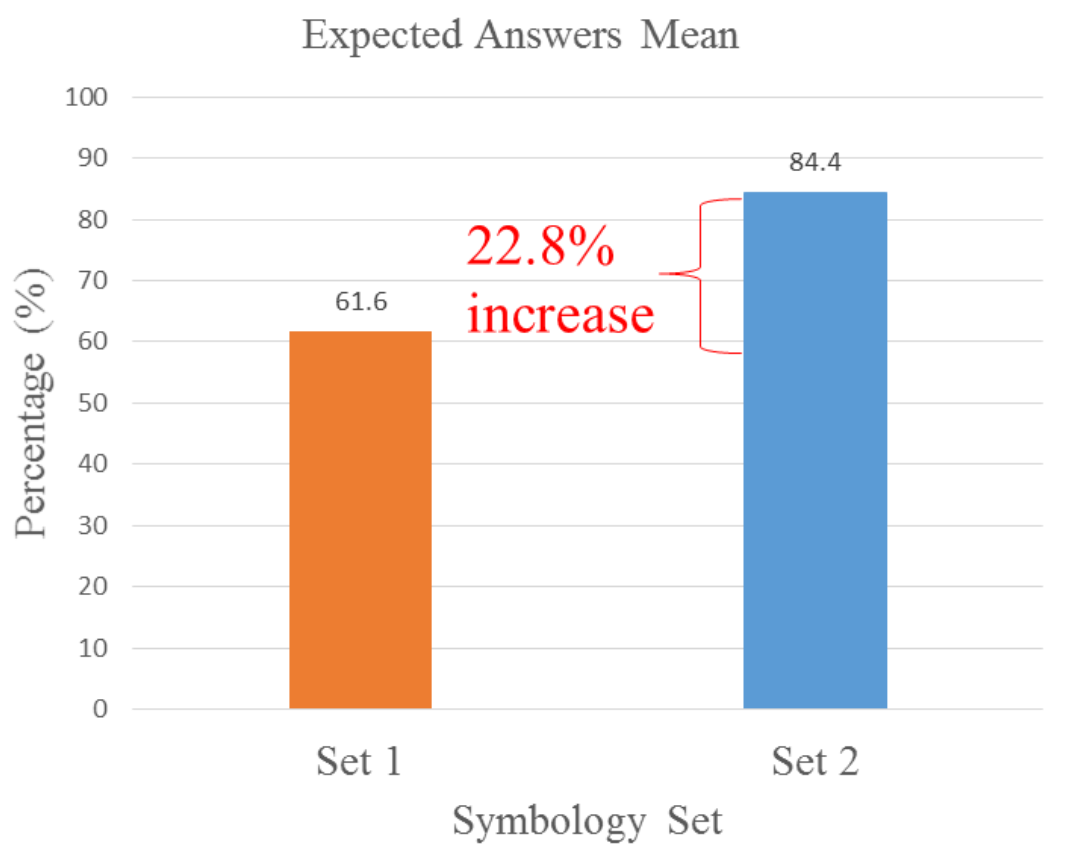

Figure 6-10: Expected Answers Mean

Note that though a substantial increase, a small sample size of only 29 participants may have influenced the results. A larger sample would provide results that are more accurate, and the survey should be administered on various samples if given the opportunity. However, these results provide a basis for understanding the importance of map symbology for clearer interpretation.

Each symbol tested twice against another symbol, and participates may have deduced which one was duplicated in the second question. Testing each symbology at least twice was necessary to determine the intuitiveness of the symbol. The questions were also in a prescribed order since the survey could not automatically randomize the questions with respect to the survey sections. If administered again, the questions should also be randomized and symbology sets be tested against each other. The scatter-plots in Figure 6-11 indicate the amount of expected answers selected in each matching section of the survey. The first graph (figure a) shows the dispersed results for Set 1; the second graph (figure b) shows the results of expected answers selected in Set 2.

The results also suggest in-test learning, especially for Set 2 as the participants selected more of the expected answers in the latter half of the survey. This also proposes that participants were able to recognize the custom symbols due to their iconic designs. 


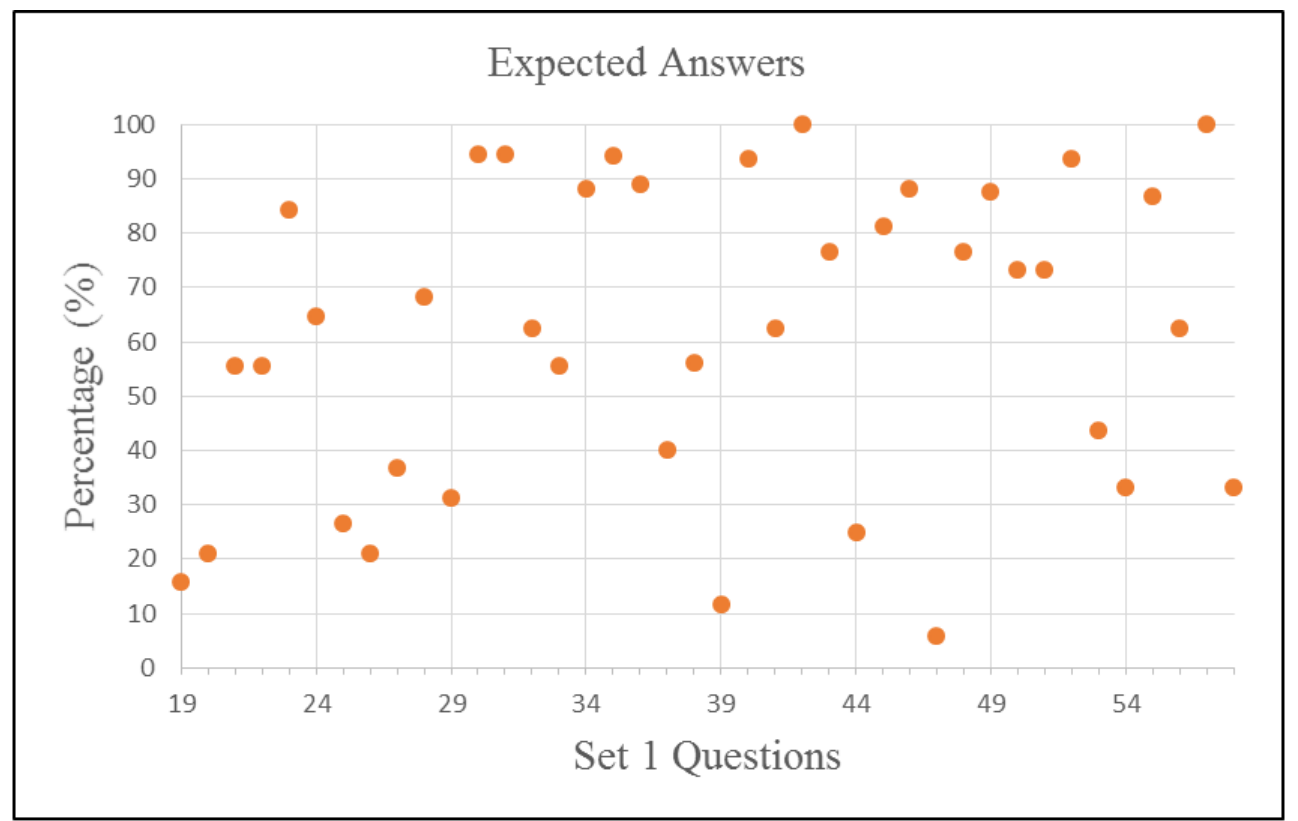

Set 1 - 18 people responded on average (11 skipped)

(a)

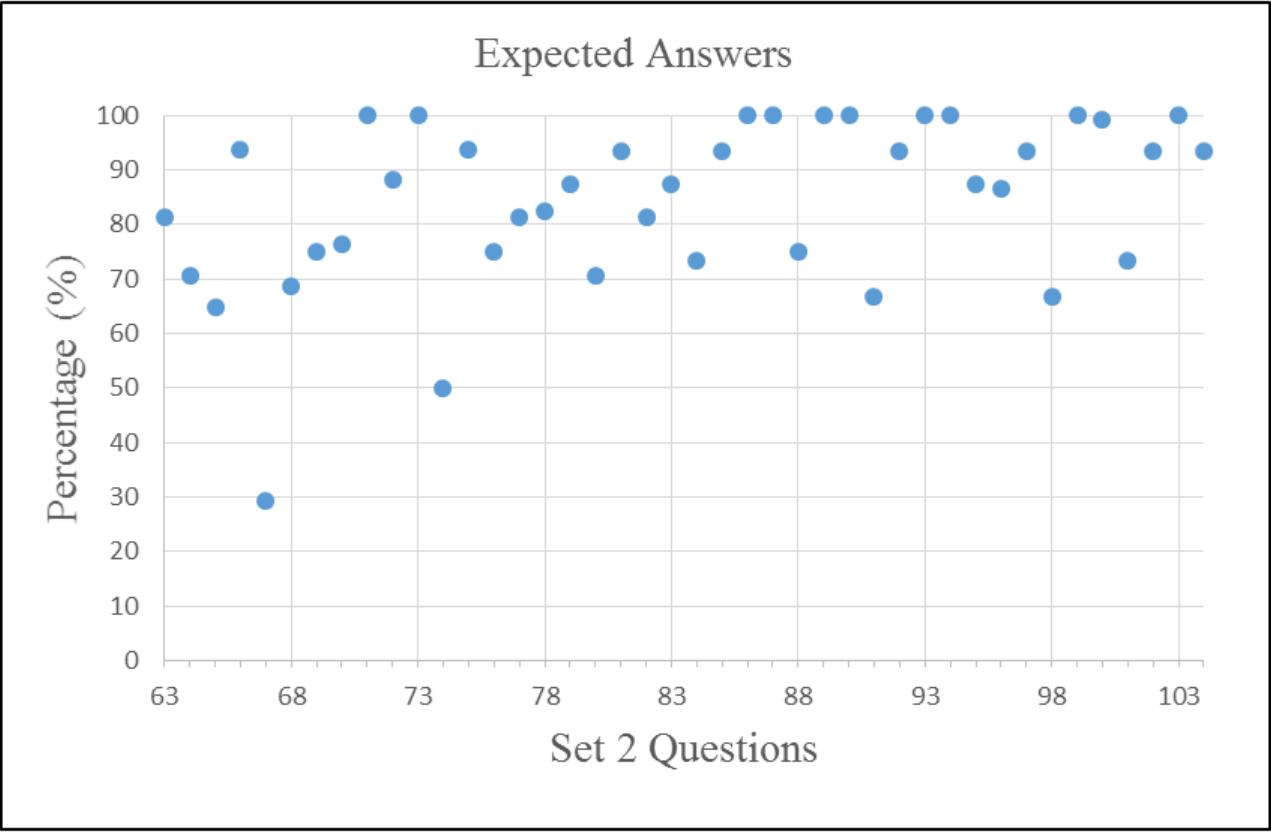

Set 2 - 16 people responded on average (13 skipped)

(b)

Figure 6-11: Scatterplot of Expected Answers Results 


\subsection{Summary}

In conclusion, all of the products satisfied the project requirements by establishing a cartographic standard for representing excavation sites. The Hopi Archaeological Set symbology improved the appearance of prior maps by using iconic symbols that represent the Hopi people. The wall and stone wall symbology produced a similar effect to the stippling technique used in traditional archaeological maps, though it is noted that the line symbology adjusts automatically at different scales. The survey results suggest that the Hopi Archaeological Set is more intuitive than the original symbology since participants selected $22.8 \%$ more of the expected answers for the custom symbology. However, only 29 subjects participated in the survey. Moreover, the 3D modeling produced a more realistic depiction of the major sites even though this aspect of the project never developed fully. 



\section{Chapter 7 - Conclusions and Future Work}

This chapter provides an overview of the project and the final products delivered to the client, as well as suggestions for potential future work topics. The central goal of this project established a cartographic standard for representing archaeological sites on the Hopi Reservation reproducible for the client to create maps of new sites. This was accomplished by producing a custom symbology set for a map series and 3D models of inferred structures. The maps communicate more effectively by using custom symbology to represent the sites, so viewers envision the archaeological ruins more accurately. This project successfully satisfied the five functional and the three nonfunctional requirements established by the client during the planning phase.

The products delivered to Dr. Bernardini included a geodatabase containing the client's fieldwork data in a standardized projection system, a symbology set that invoked the Hopi culture and archaeological features, a series of 144 maps, and fly-through 3D animations of 10 major archaeological sites. The ArcMap 10.3 File Geodatabase provided a more systematic method for managing the data and projected the data in NAD83 UTM Zone12. With consideration to the original scope and time constraints, adding three new fields noting the site name, archaeological catalog number, and a unique identifier helped standardize the attribute schemas and merge some datasets. Merging the Mungyaovi data improved the dataset's functionality and made the map design process more efficient by reducing the time spent on the symbology. The map series produced grayscale and color versions of each site for potential publication in the client's future work. Individually exported layer produced PDF files that allow the client to select what layers are visible within the document. Dr. Bernardini also acquired the symbology set and templates used to make the map series, so this cartographic standard is applied to maps designed in the future. The symbology set encompassed 22 archaeological features with design inspiration from Hopi artifacts and traditional archaeological maps. This created unique visualizations for representing archaeological sites and gives character to the client's fieldwork data. The survey results indicated that the symbol set was more intuitive than the default symbol used prior. However, some elements may be expanded. The following sections discuss potential topics for future work that can use the results of this project as a catalyst.

\subsection{Complex 3D Models}

Additional symbols can be incorporated into the existing symbology set to produce a collection of symbols that encompasses all of the artifacts found in the input data. This includes kivas, burials, fences, and historical sites. Expanding on how 3D models represent sites is another potential topic. From a cartographic perspective, the 3D models may include floor elevations and realistic symbology for artifacts, such as a hearth, to generate a more intriguing virtual-reality model. Analytically, the 3D models may provide a detailed analysis for horizon lines and an understanding in how the Hopi viewed the surrounding landscape. The "Floors" fields require editing before continuing with this potential project addition, so the heights of the structures are accurate. Observing the orientation of objects and their spatial relationships may also open new 
avenues for 3D archaeological research. Another option for expansion is producing 3D temporal models that reflect the time in which the Hopi constructed structures and surrounding objects, and by observing how artifacts are super-transposed in the subterrain. To do this, temporal records and elevations for each feature in the feature class need to be added to the existing data; such an analysis is worth investigating since it could improve excavation efforts.

\subsection{Relief Representation}

Many of the contour lines in the input data were oversimplified, inconsistent, and did not provide a detailed view of the topology surrounding the sites. Sample elevation points collected by GPS generated the input contour lines. Though functional, this process simplifies the actual topology depending on where and how many elevation points were collected. The outlier points may also generate overly exaggerated contour lines while the interior points produce a more actuate depiction of the landscape. The default topology basemap hosted in ArcMap is too simplistic for the large scales needed to show the excavation sites at a detailed level. One option is to expand on relief representation by using Lidar to extract contour lines from datasets containing a multitude of elevation points. Lidar would provide a detailed view of the geography to incorporate into the existing map series. However, since this is often expensive, many researchers opt for collecting GPS elevations during fieldwork. Another option is to use the Digital Globe Foundation imagery received for this project and determine if there is a method for extracting the contours. Considering relief representation would enhance the way the maps portray the geography and may prove more useful during fieldwork.

\subsection{System Design}

The last potential addition is to reconsider the system design of the geodatabase. The client recorded data individually for each archaeological site. The current geodatabase reflects this collection method by having each feature dataset contain information about each site. The limitation of this design is that attribute schemas between similar features on different sites vary and are becoming more difficult to manage. Relationships between objects are unobserved in this manner without performing the same spatial analysis process multiple times on different datasets. Another system design could merge the individual sites together so there are fewer files to manage. Domains for site names, catalog numbers, or collection unit titles can be set within the attribute fields to improve the integrity of the data, and feature classes retaining the same attribute schema should also be determined. It is highly recommended that the integrity of the data be the foremost concern for future work. 


\section{Works Cited}

Booth, B. (2005). Archaeological Symbol Set. Retrieved from Esri Forum: (need to find the URL)

Brewer, C. A. (2005). Designing Better Maps. Redlands, CA: Esri Press.

Cal Fire. (2012). CAL FIRE Archaeology Program. Retrieved from http://calfire.ca.gov/resource_mgt/archaeology-history

Cutright-Smith, E. (2013). Mapping Ancestral Hopi Archaeological Landscapes: An Assessment of the Efficacy of GIS Analysis for Interpreting Indigenous Cultural Landscapes. Tucson, AZ: University of Arizona.

Dent, B. D. (1985). Principles of Thematic Map Design. Reading, MA: Addison-Wesley Publishing.

Drap, P., Seinturier, G., Conte, A., Caiti, D., Scaradozzi, S., Zanoli, M. \& Gambogi, P. (2008). Underwater Cartography for Archaeology in the VENUS Project. Geomatica 62(4), pp. 419-427. Retrieved 10/26/2015 from http://s3.amazonaws.com/academia.edu.documents/29559410/Geomatica08_venu s.pdf?AWSAccess KeyId=AKIAJ56TQJRTWSMTNPEA\&Expires=1445890921 $\&$ Signature=\%2By6y0GiLYN5thQeauS5nWi8B9ic\%3D\&response-contentdisposition=inline \%3B\%20filename\%3DUnderwater_cartography_for_archaeolo gy_i.pdf

Harris, T. M. (2002). GIS in Archaeology. In A. K. (Ed.), Past Time, Past Place: GIS for History (pp. 131 - 143). Redlands, CA: Esri Press.

Howard, P. (2007). Archaeological Surveying and Mapping: Recording and Depicting the Landscape. New York, NY: Routledge.

Imhof, E. (2007). Cartographic Relief Presentation. Redlands, CA: Esri Press.

Keightly, K. (n.d.). Cutural Resource Data Management and Mapping Using GIS: A Unified Approach for a Large Scale FERC Relicensing Project (PowerPoint). Retrieved from http://proceedings.esri.com/library/userconf/proc15/papers/990_159.pdf

Knowles, A. (2005). GIS and History. In A. K. (Ed.), Placing History: HowMaps, Spatial Data, and GIS are Changing Historical Scholarship (pp. 1 - 25). Redlands, CA: Esri Press.

Kvamme, K. L. (2012). Spatial Information Technologies and Landscape: Past Problems and Future Directions. eTopoi: Journal For Ancient Studies vol. 3, 335 - 340.

Robinson, A. (1952). The Look of Maps: An Examination of Cartographic Design. Redlands, CA: Esri Press.

Robinson, A. H. (1995). Elements of Cartography (6th edition). New York, NY: John Wiley \& Sons, Inc.

Smithsonian Institution Bureau of Ethnology. (1880). Map of Payupki. In Annual report of the Bureau of Ethnology to the Secretary of the Smithsonian Institution (p. 59). Washington: G.P.O.: Smithsonian Institution.

Tufte, E. R. (1983). The Visual Display of Quantitative Information. Cheshire, CN: Graphics Press. 



\section{Appendix A. Map Series}




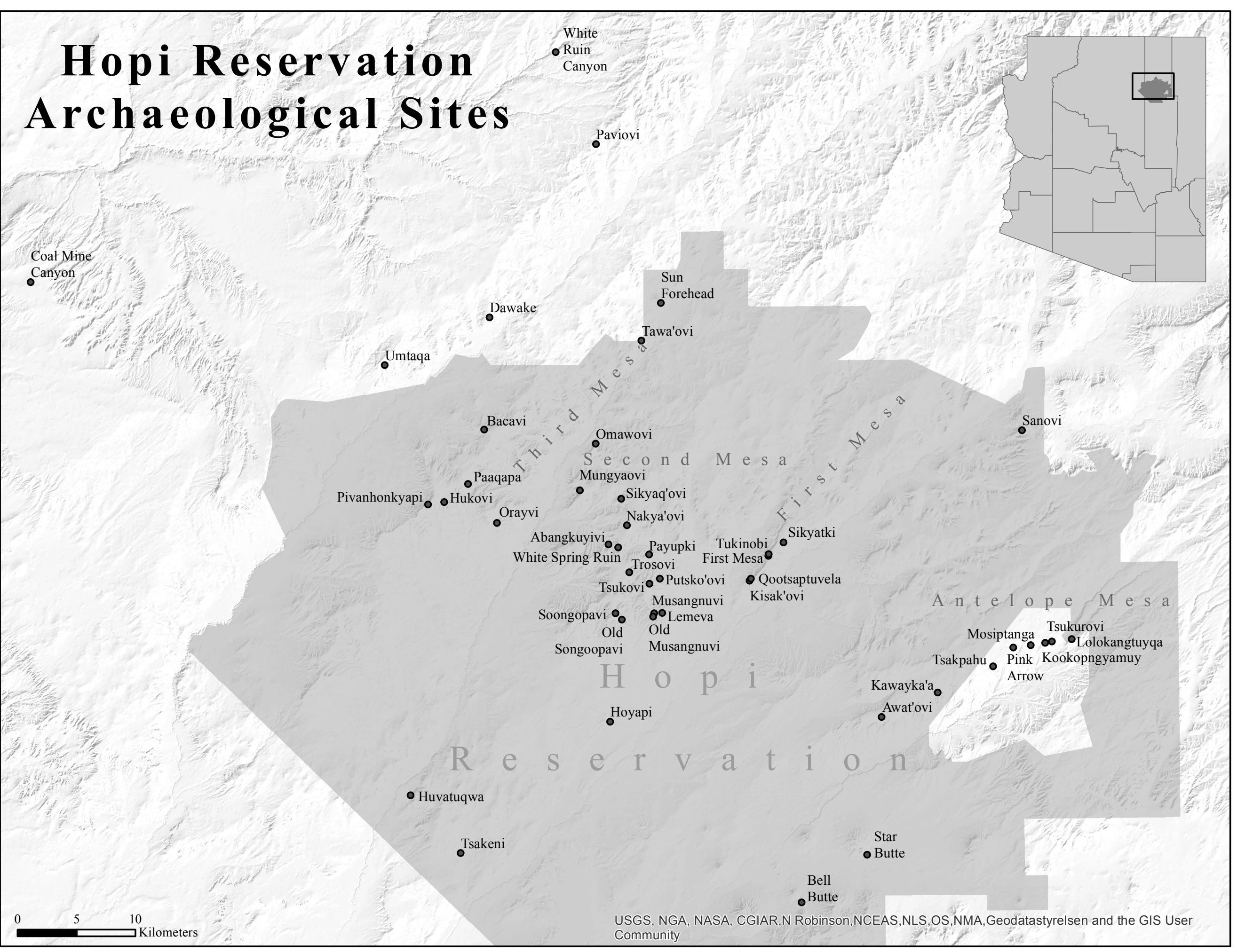




\section{Abangkuyivi}

NA2299

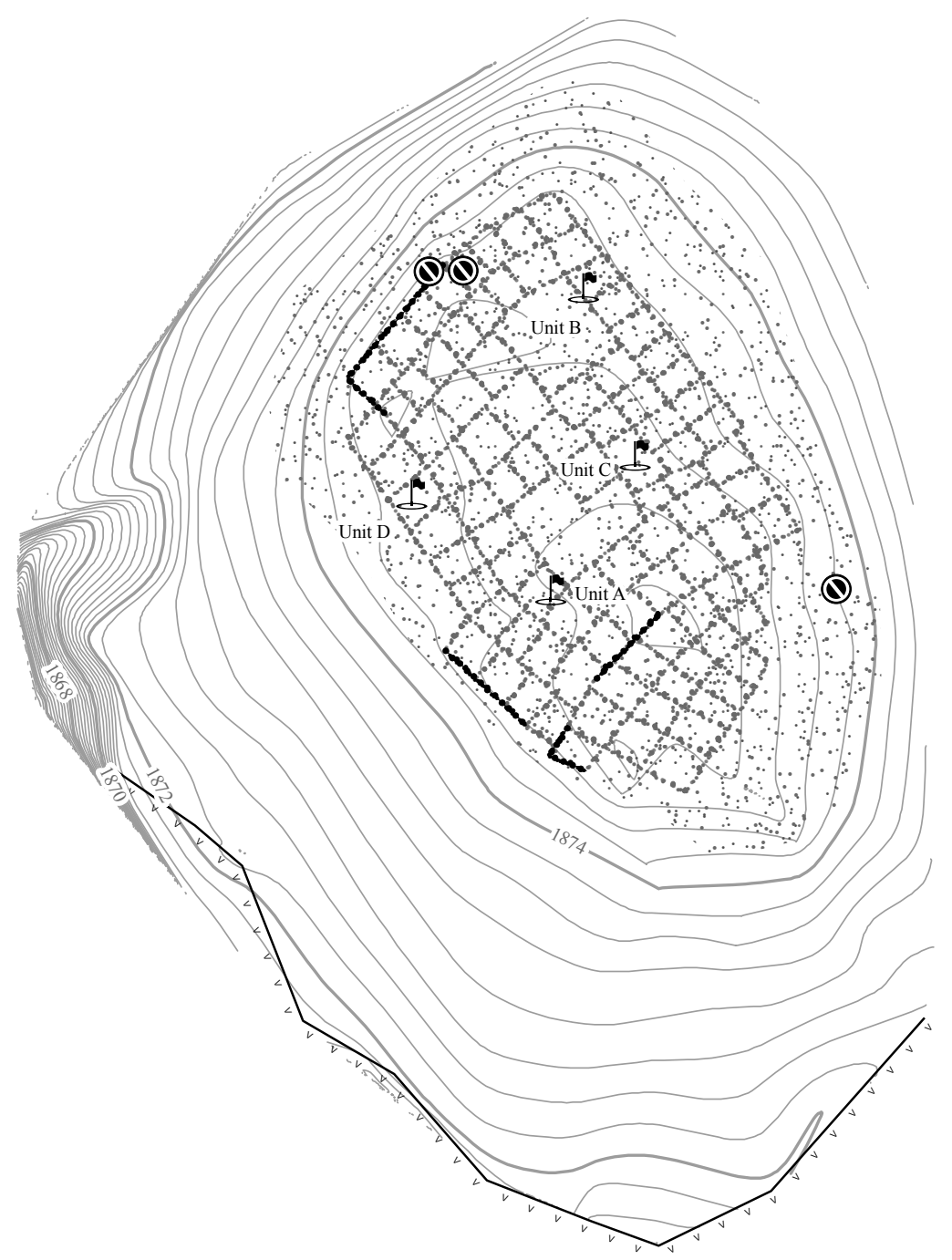

• Collection Unit

(a) Pothole

$\checkmark v \vee$ Cliff Edge

Wall

Inferred Wall

$\stackrel{N}{N}$

Rubble 


\begin{tabular}{|l|l|}
\hline Awat'ovi NA820 \\
\hline
\end{tabular}






\section{Bacavi Survey}

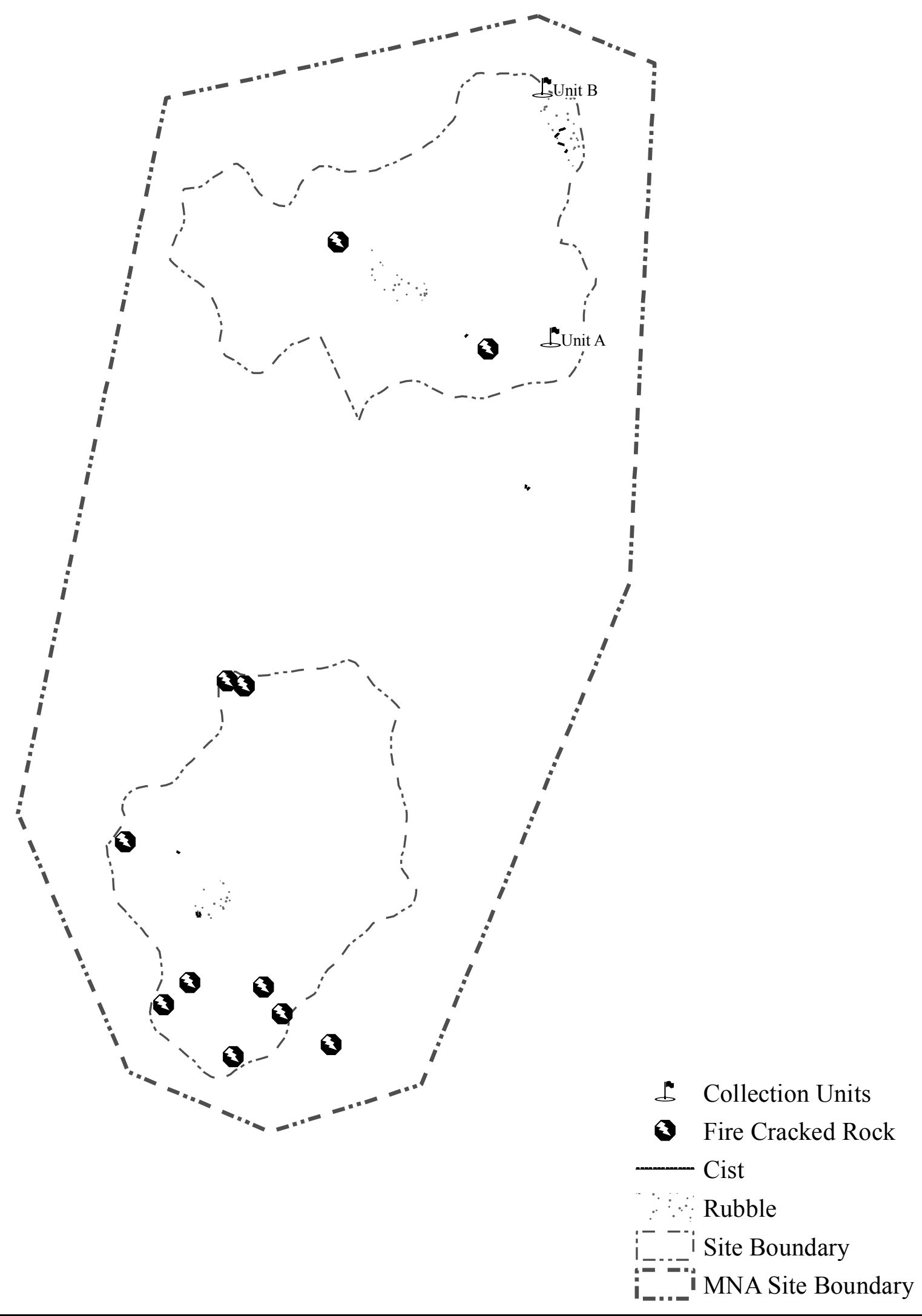




\section{Bell Butte \\ NA29240}

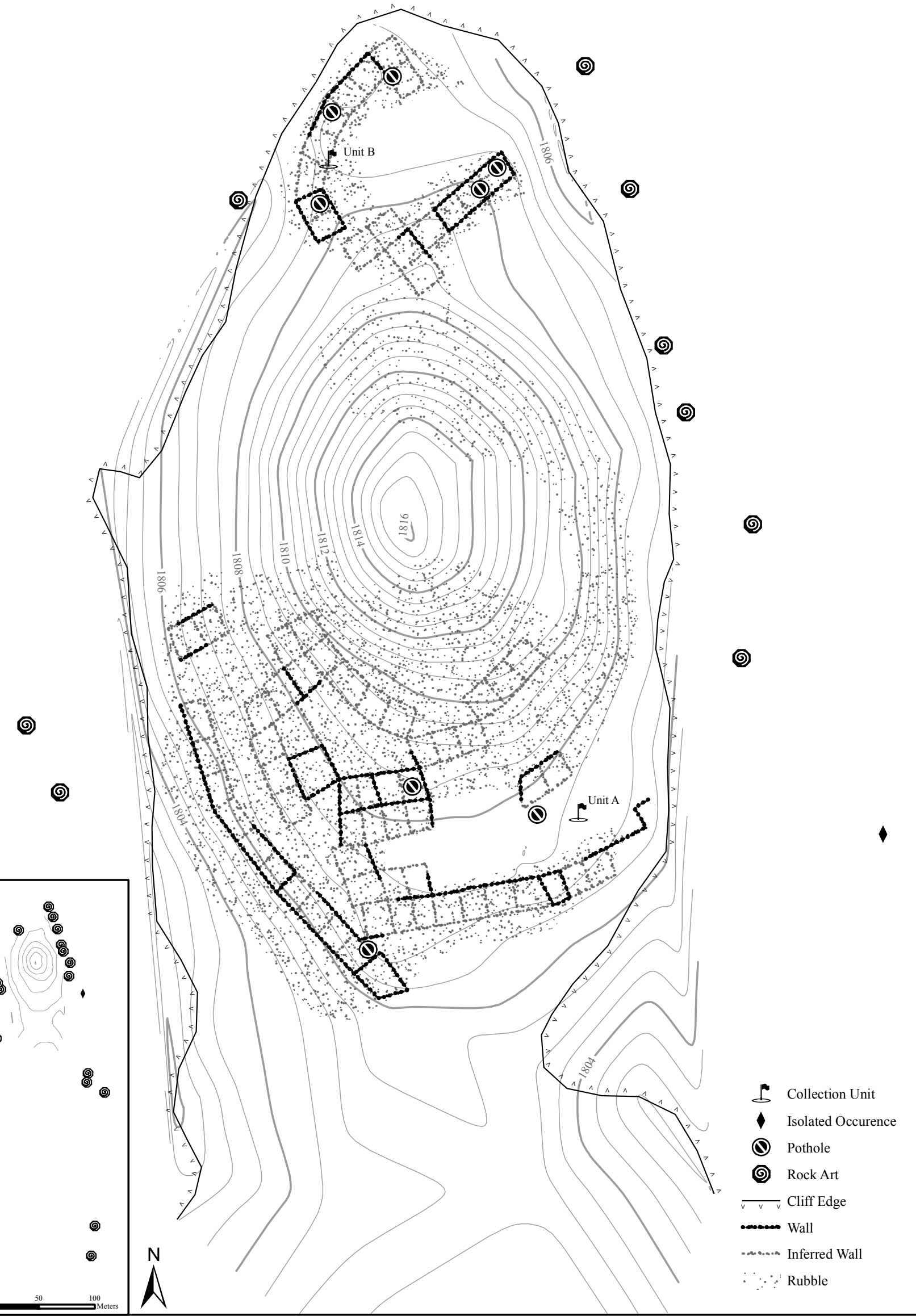




\section{Coal Mine Canyon \\ NA30505}

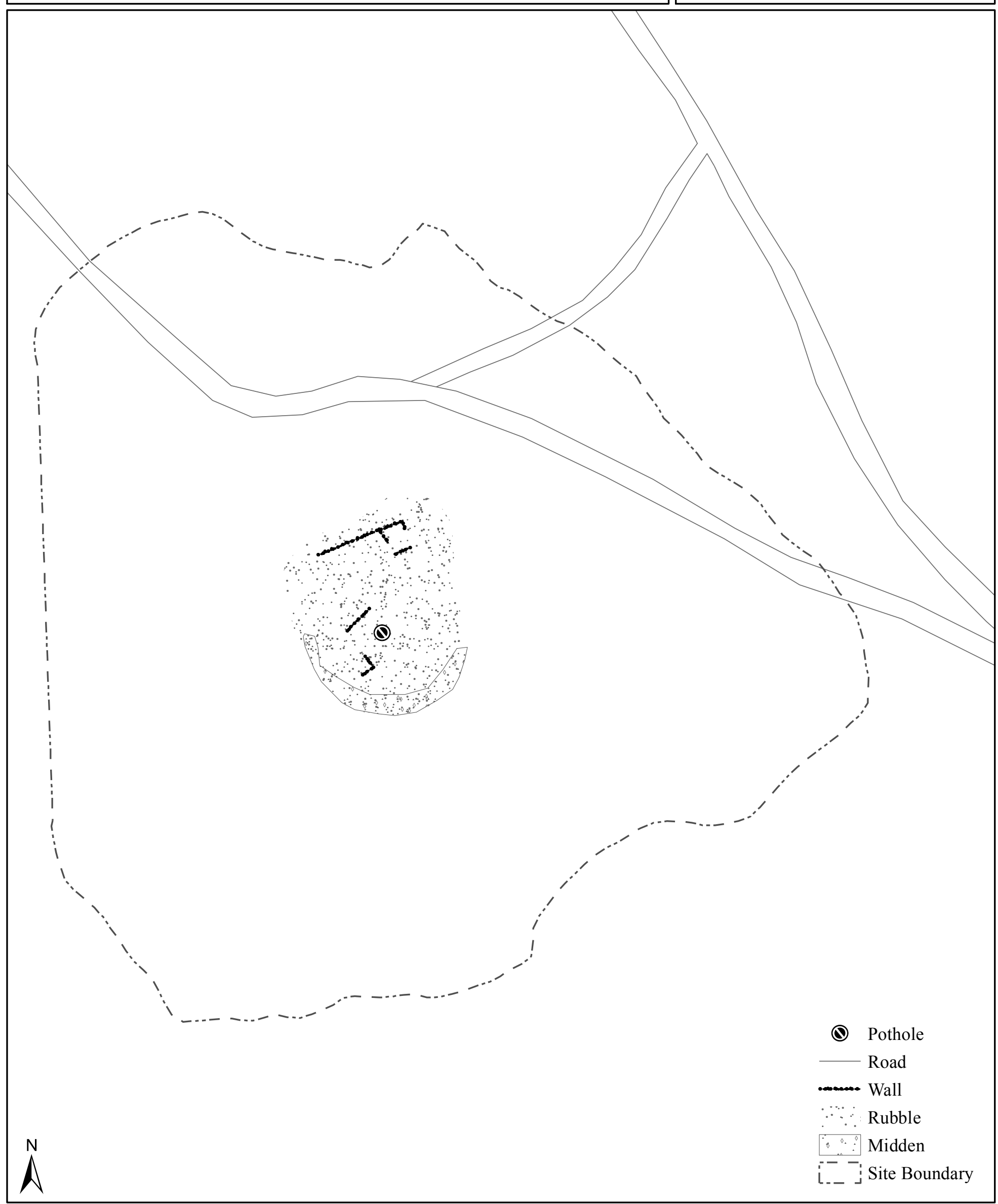




\section{Coyote Springs Survey}

NA30642; NA30643; NA3093

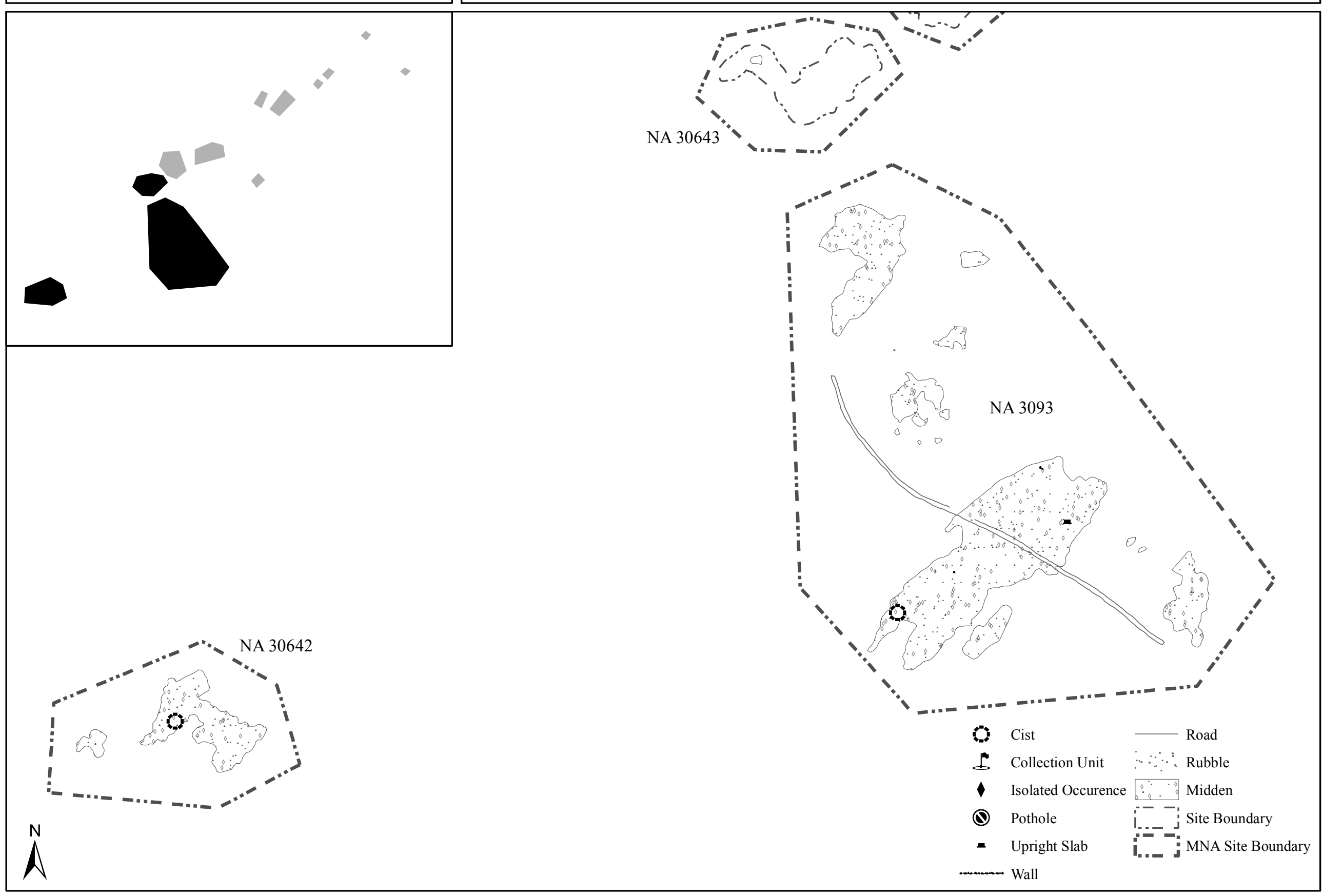

50 


\section{Coyote Springs Survey NA30643 - NA30650}

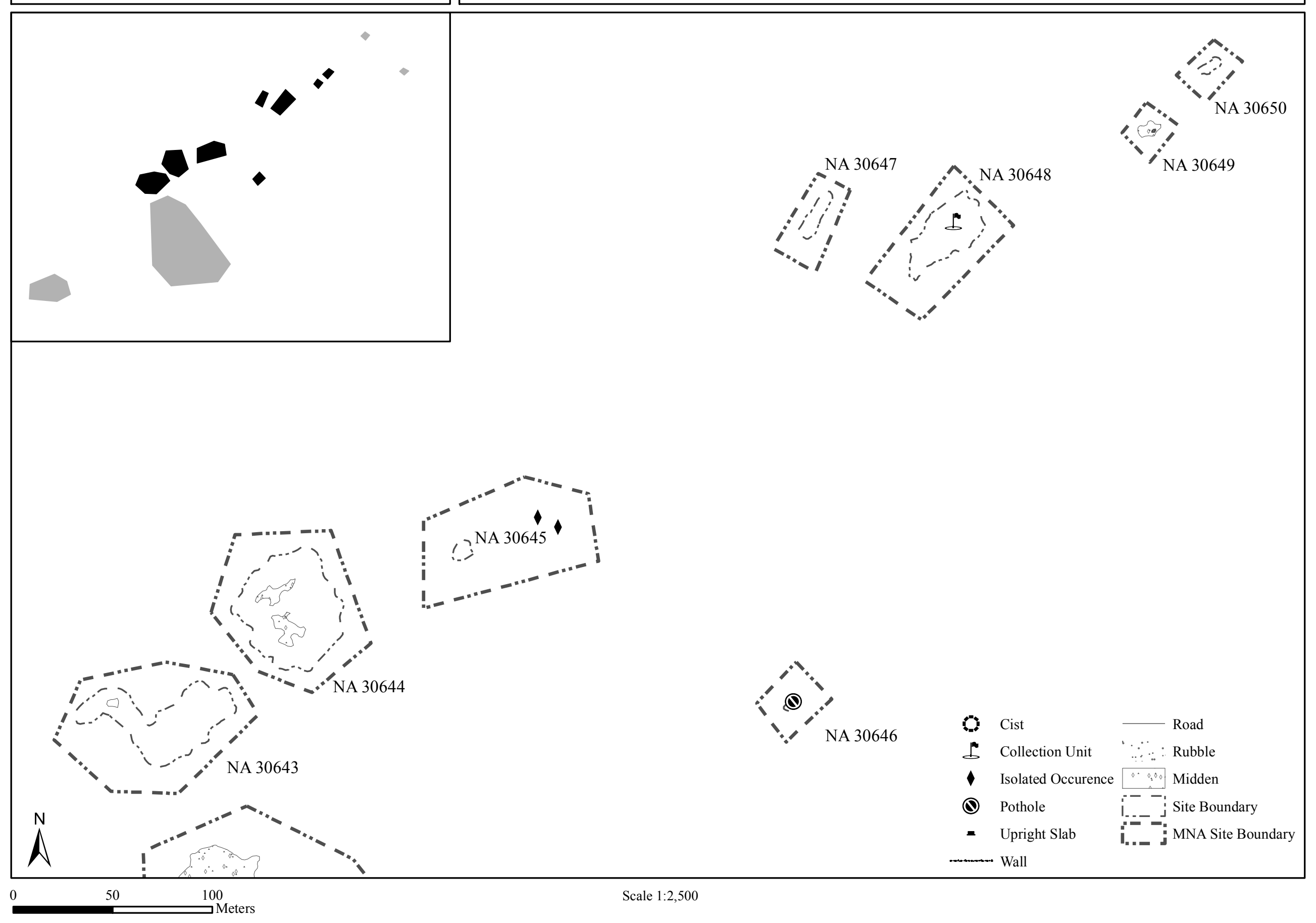




\section{Coyote Springs Survey NA30649 - NA30652}

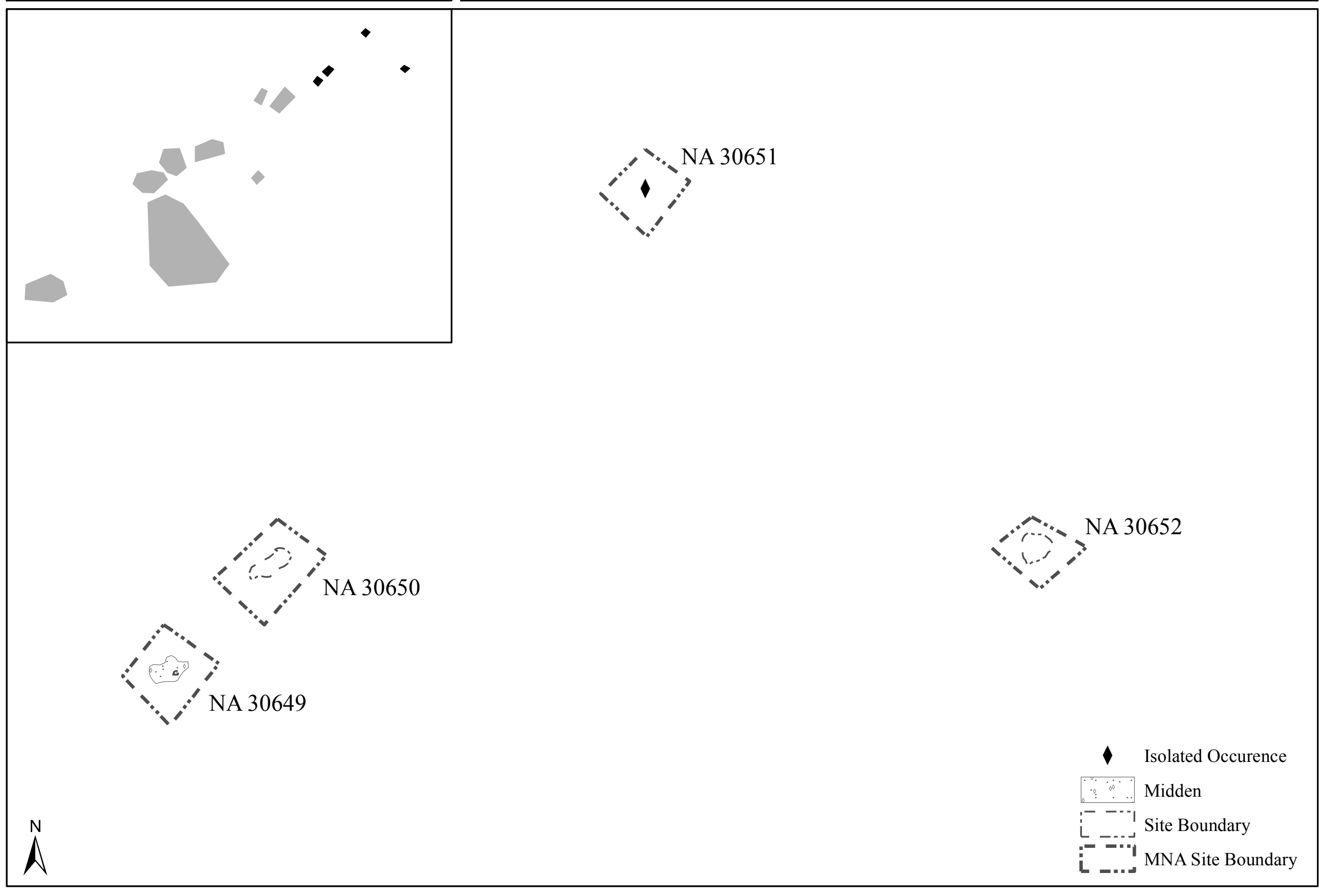




\section{Dawa Survey NA30473 - NA30475}

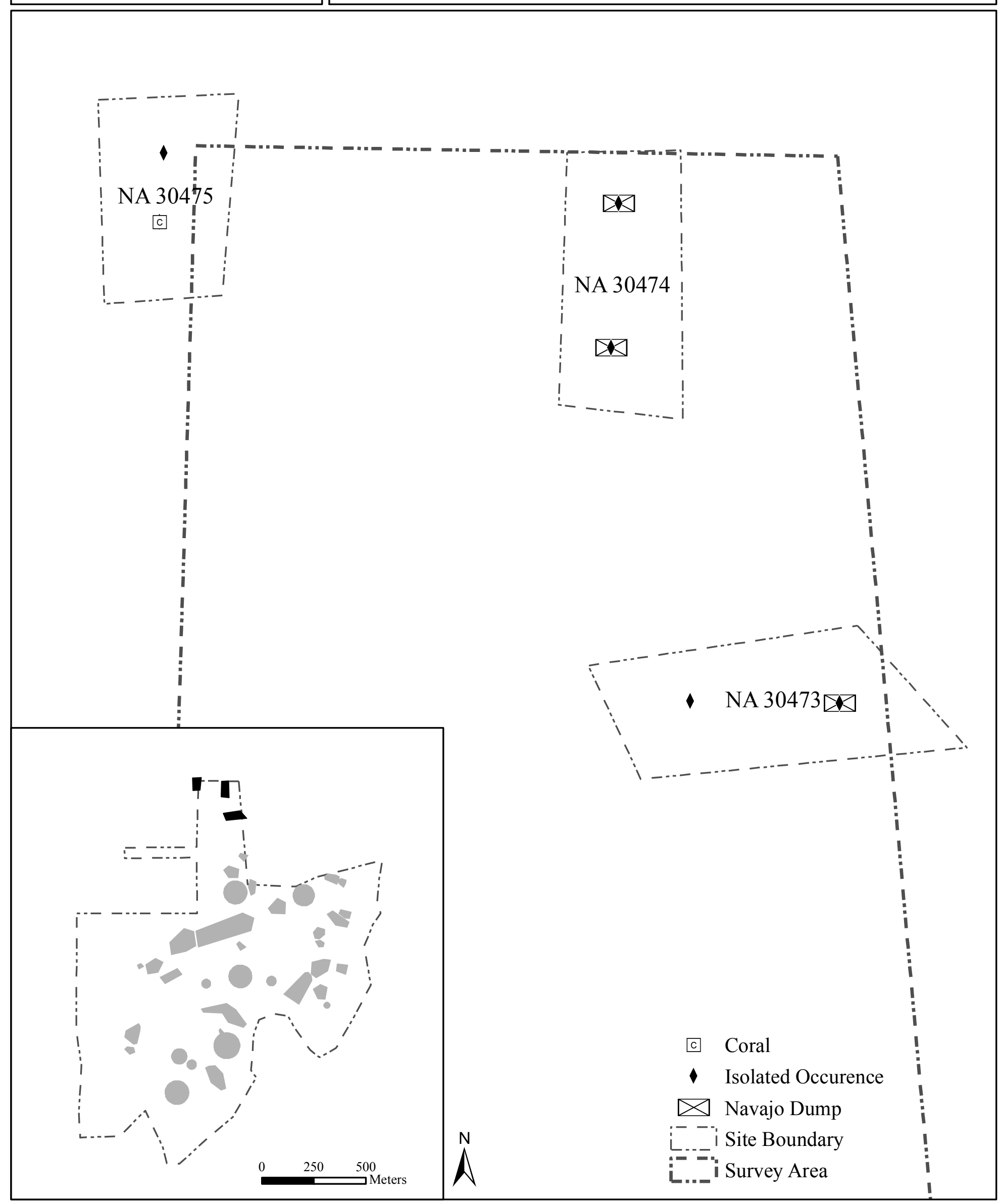




\section{Dawa Survey}

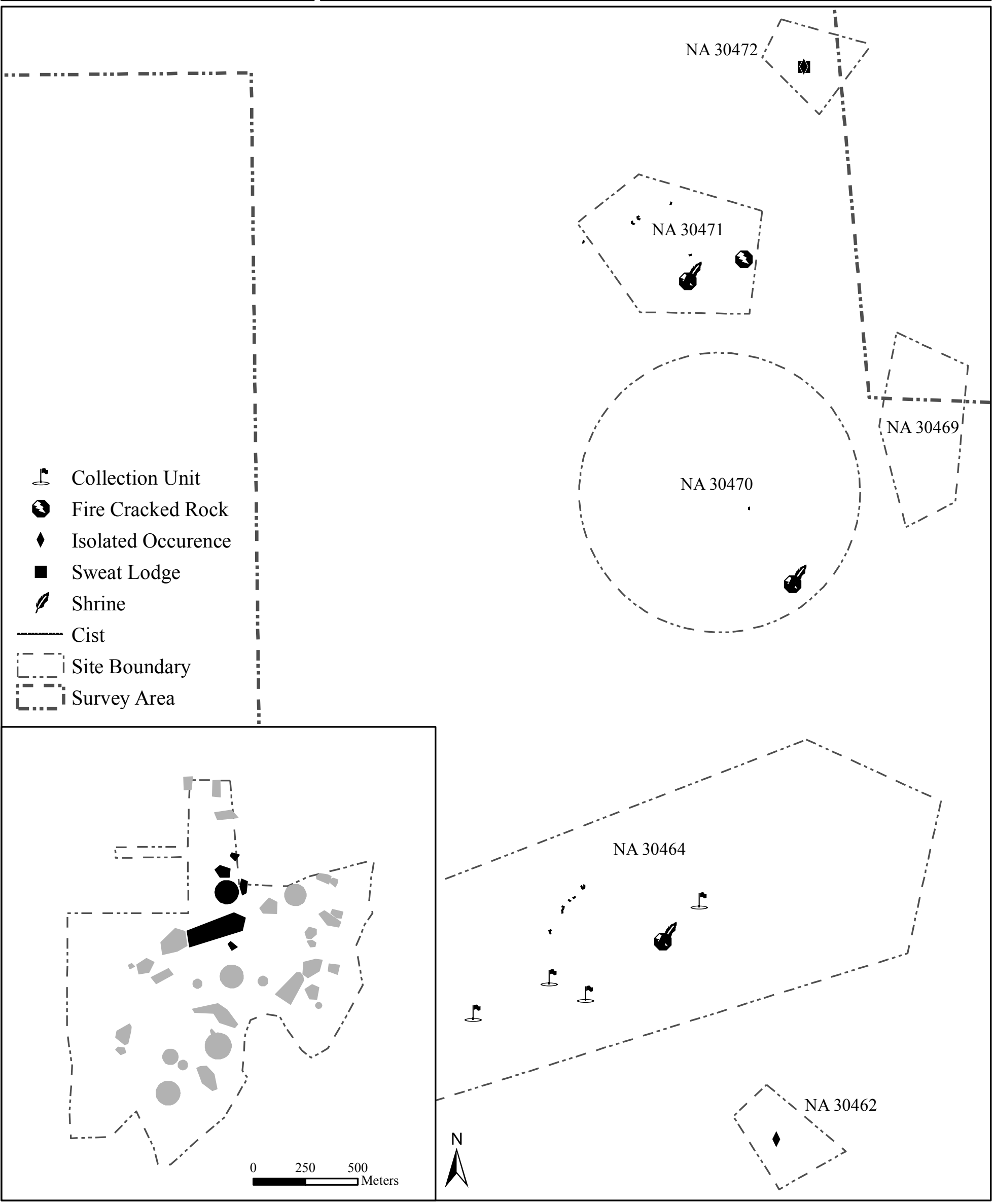




\section{Dawa Survey NA30453; NA30455 - NA30461; NA30463}

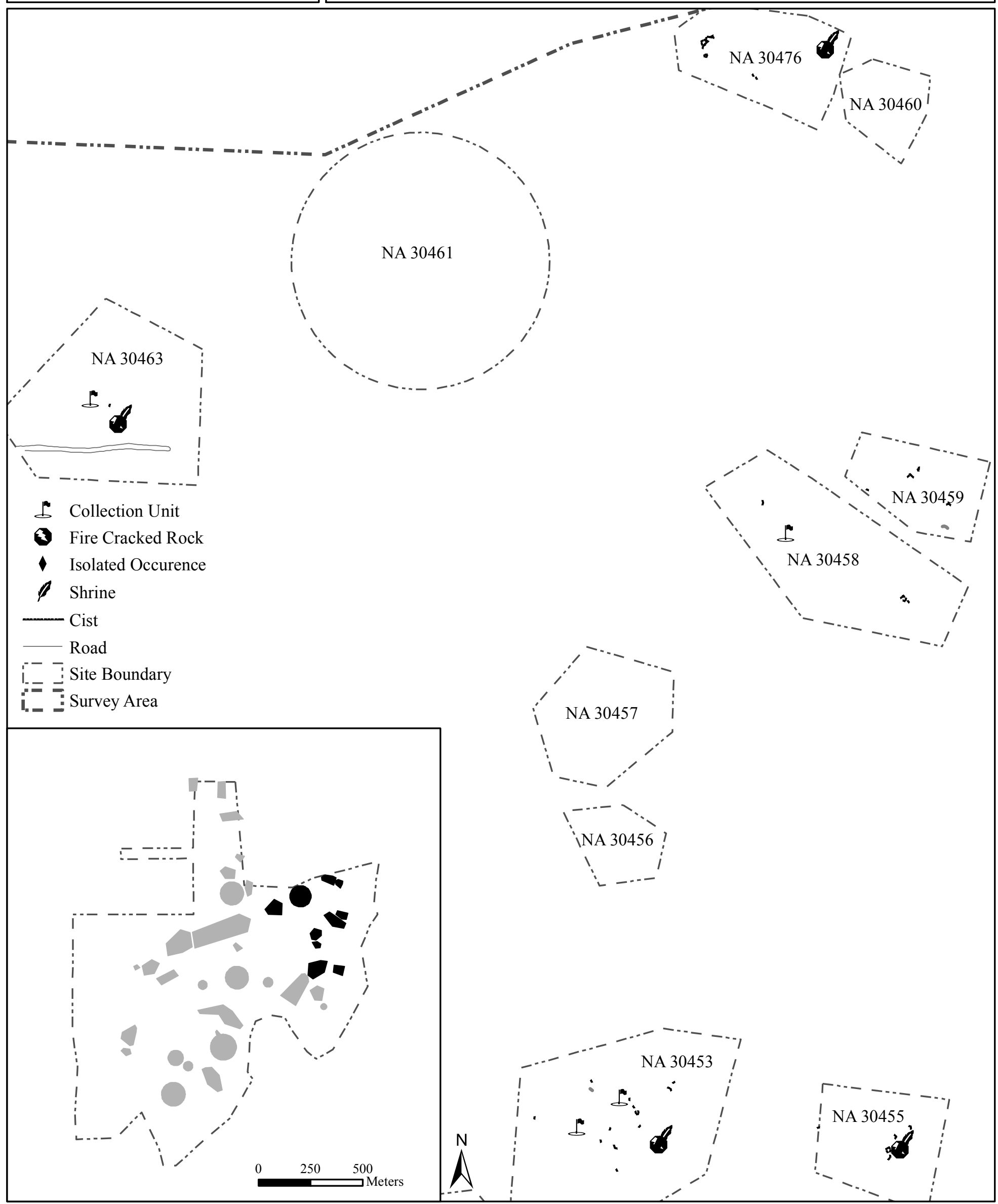




\section{Dawa Survey NA30446; NA30451 - NA30455}

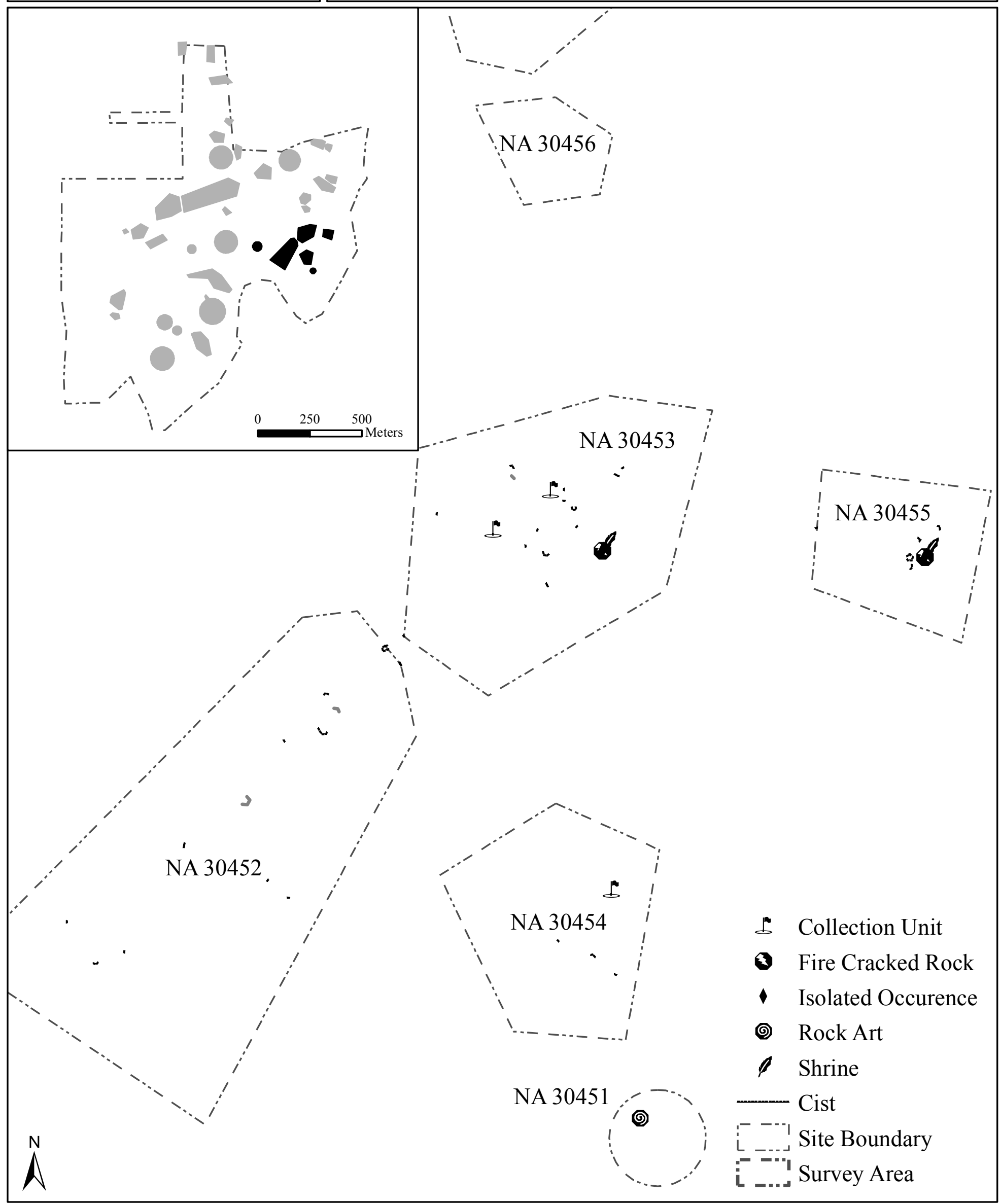




\section{Dawa Survey NA30265; NA30445; NA30449; NA30450}
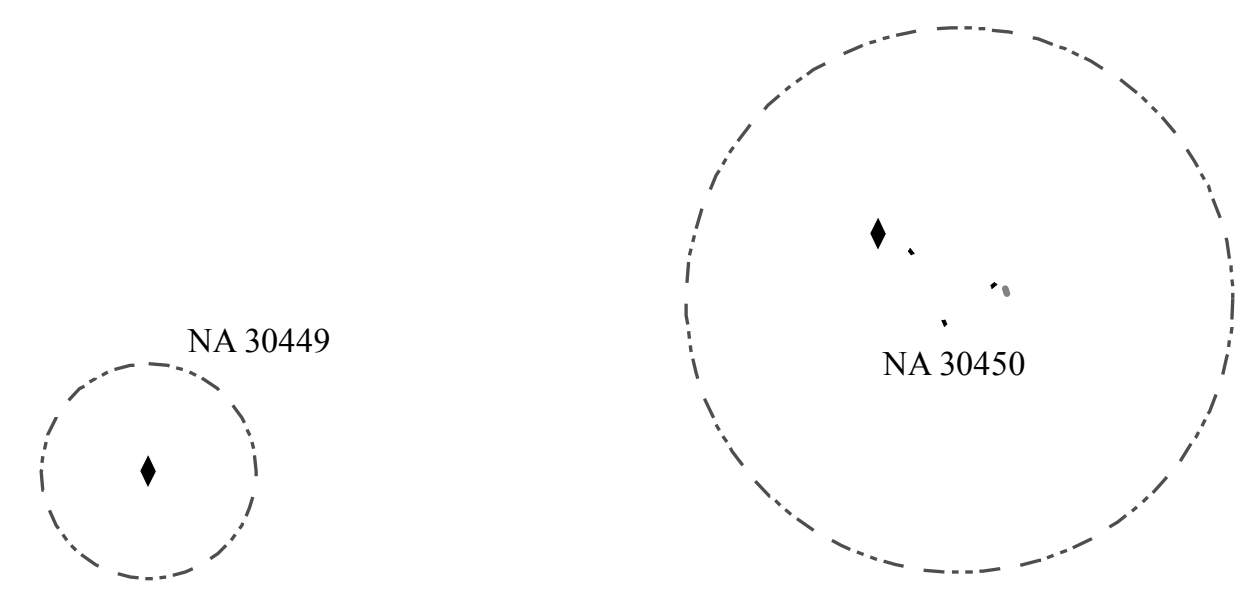

¿ Collection Unit

- Isolated Occurence

(2) Rock Art

Cist

[-..- Site Boundary

Survey Area
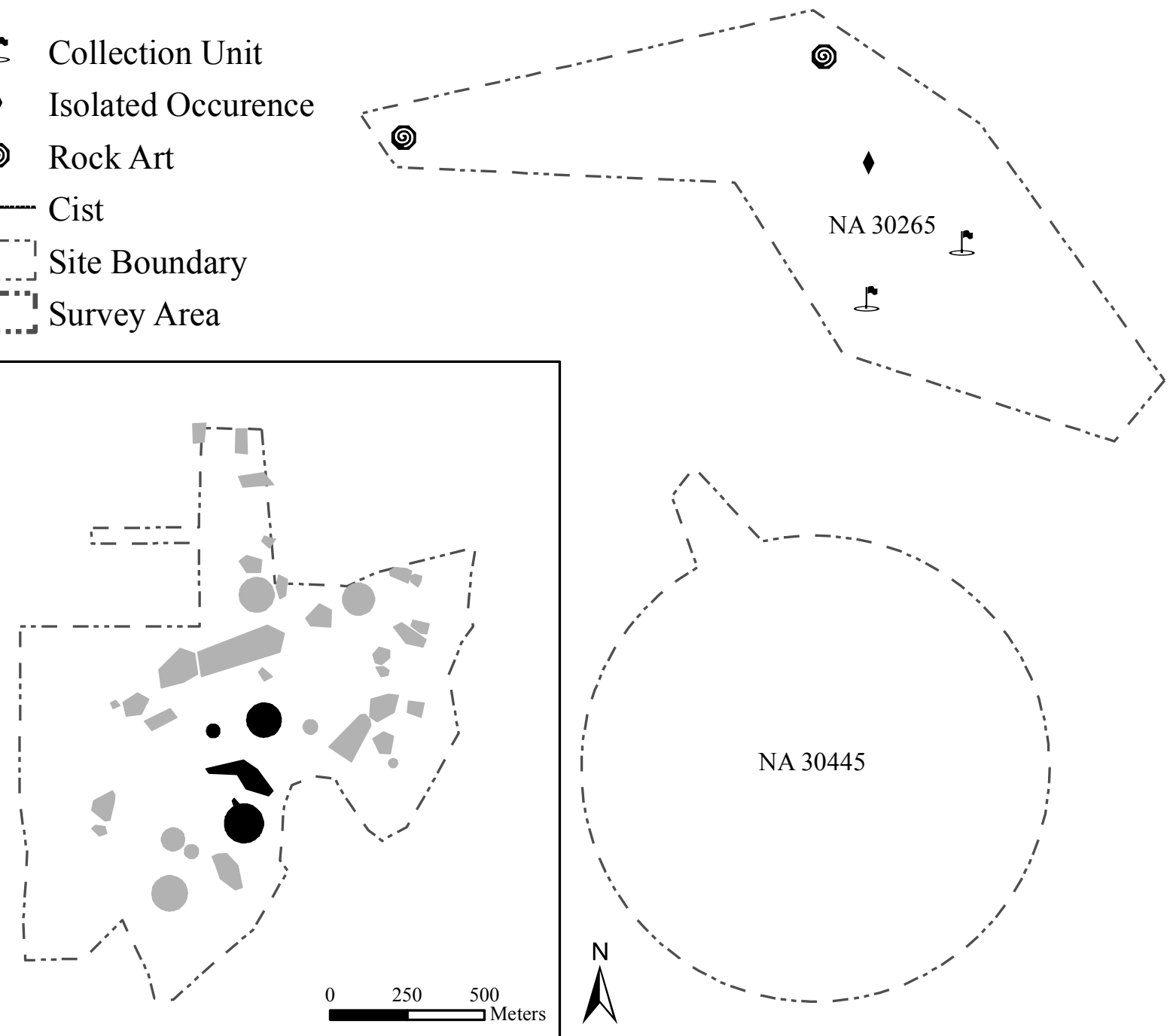


\section{Dawa Survey NA30465 - NA30468}

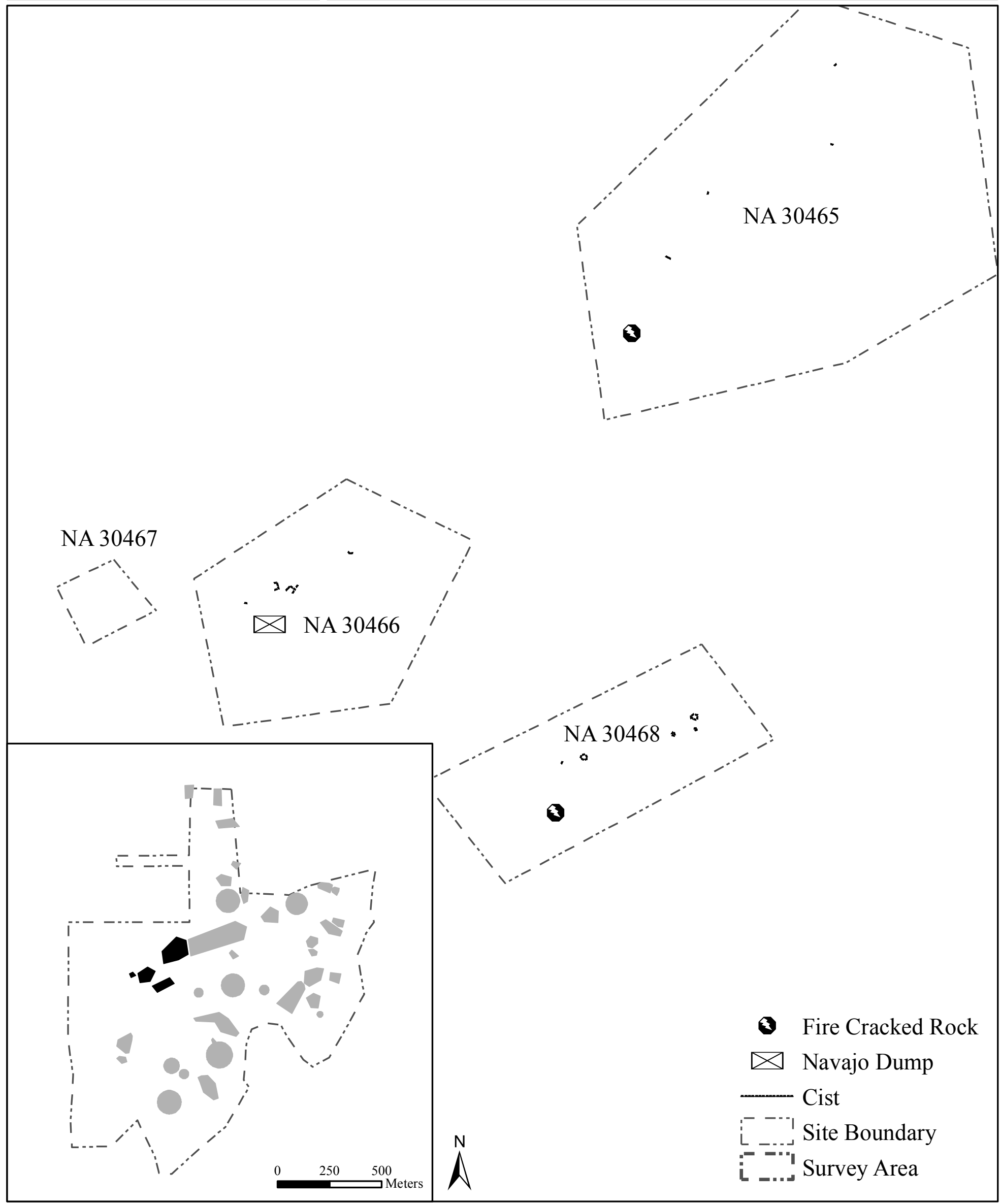




\section{Dawa Survey NA30261 - NA30264; NA30447; NA30448}

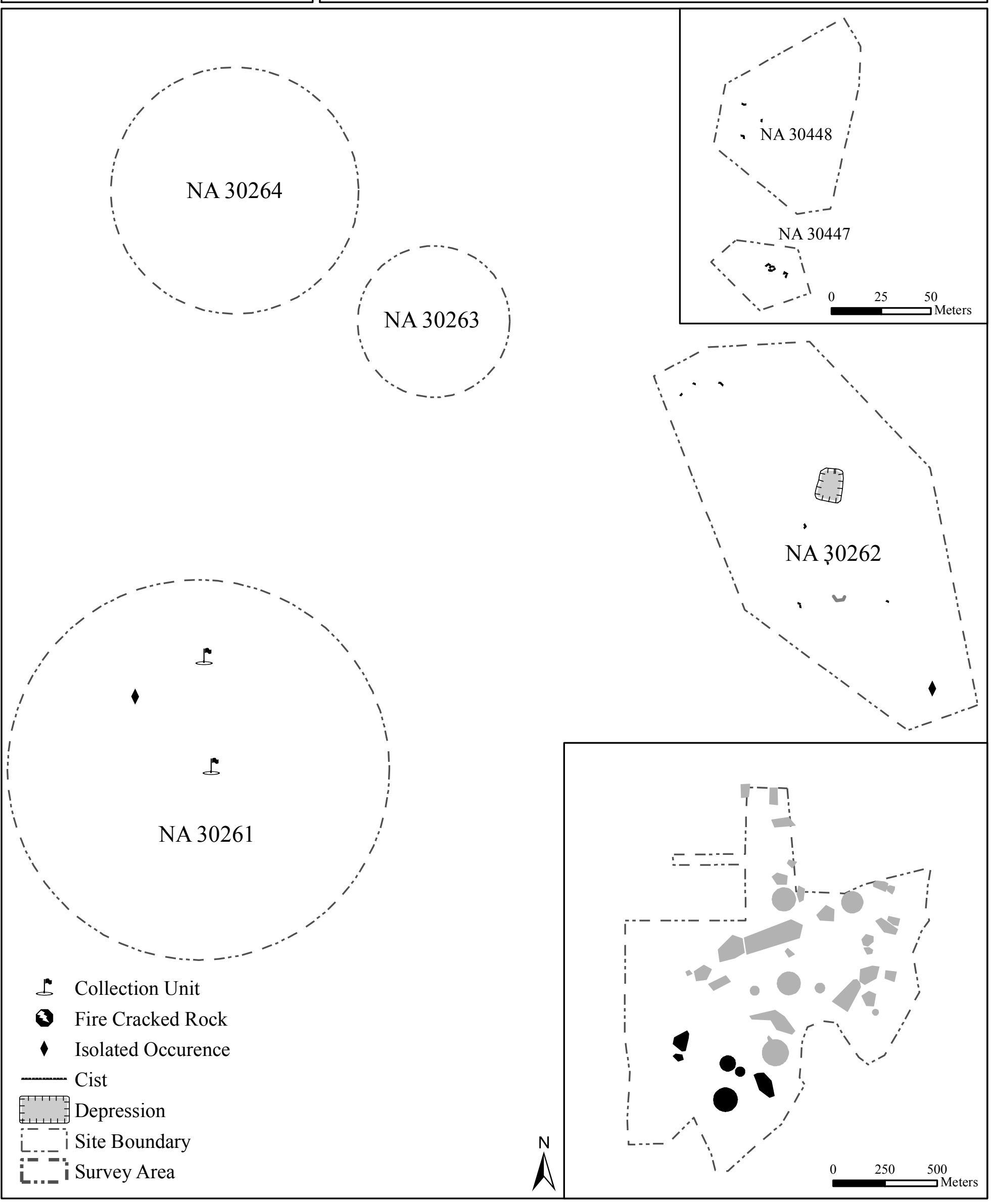




\section{\begin{tabular}{l||l} 
Dawake Survey & NA30653 - NA30659
\end{tabular}}

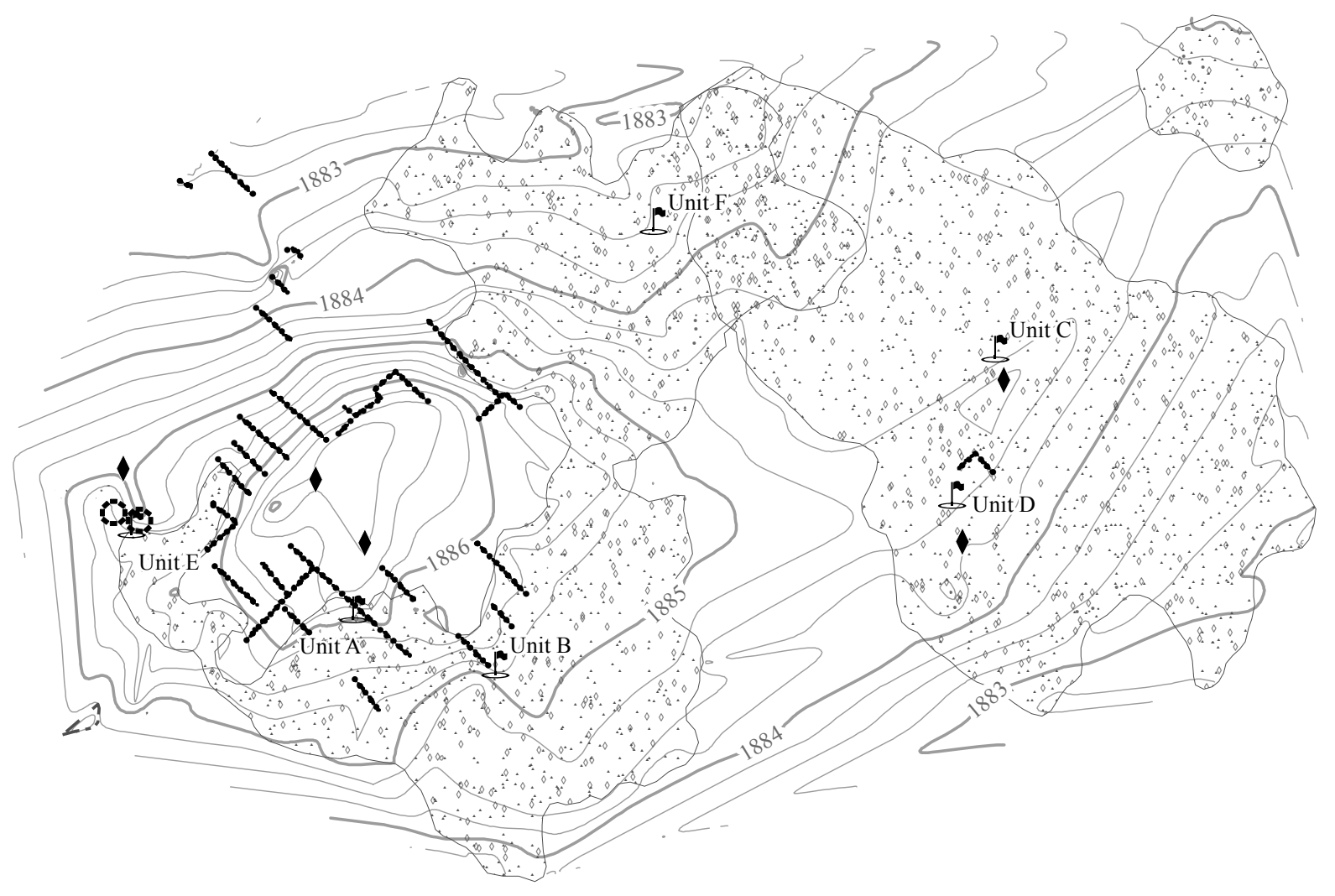

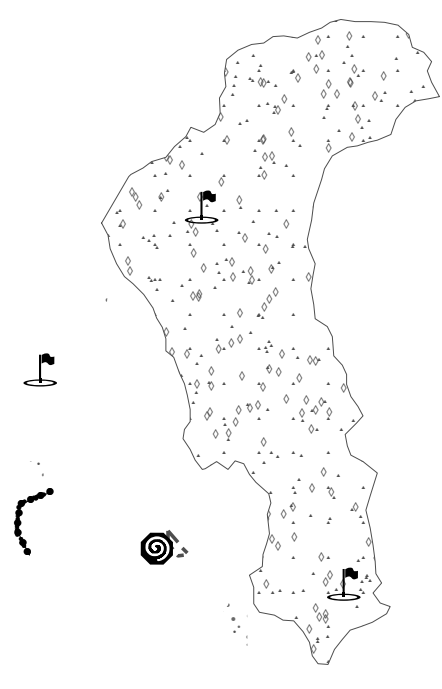

$i$

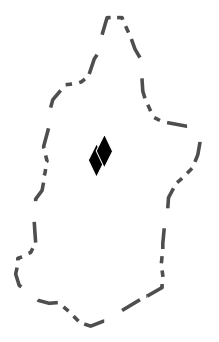

$\stackrel{N}{\Lambda}$

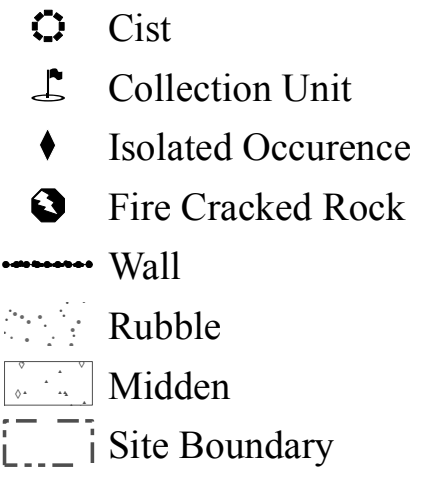

Cist

Collection Unit

Isolated Occurence

Fire Cracked Rock

Rubble

L.. i Site Boundary 


\section{First Mesa Survey}

\section{NA30171; NA30488; NA30489}

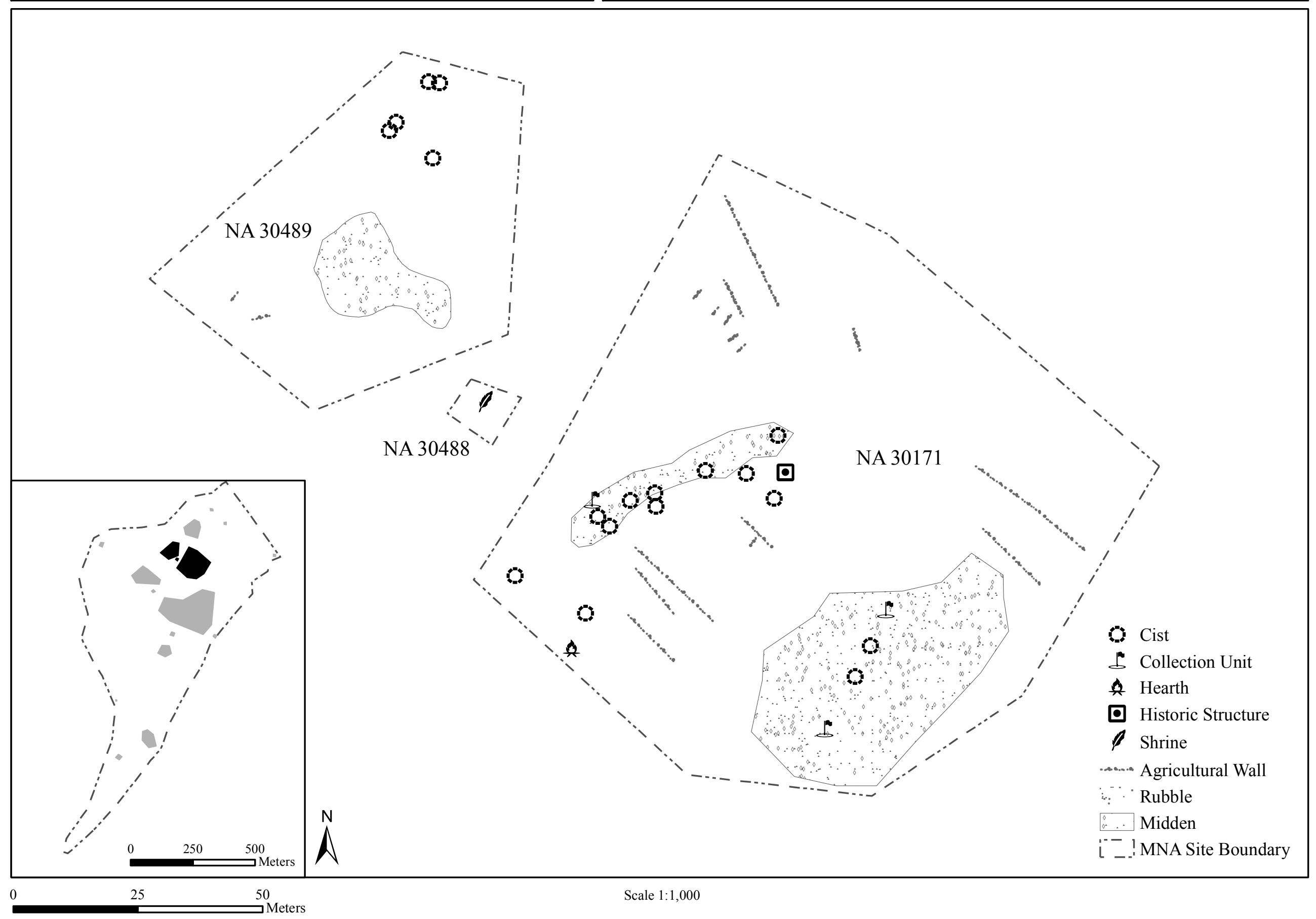




\section{First Mesa Survey}

NA30490; NA30491; NA30492

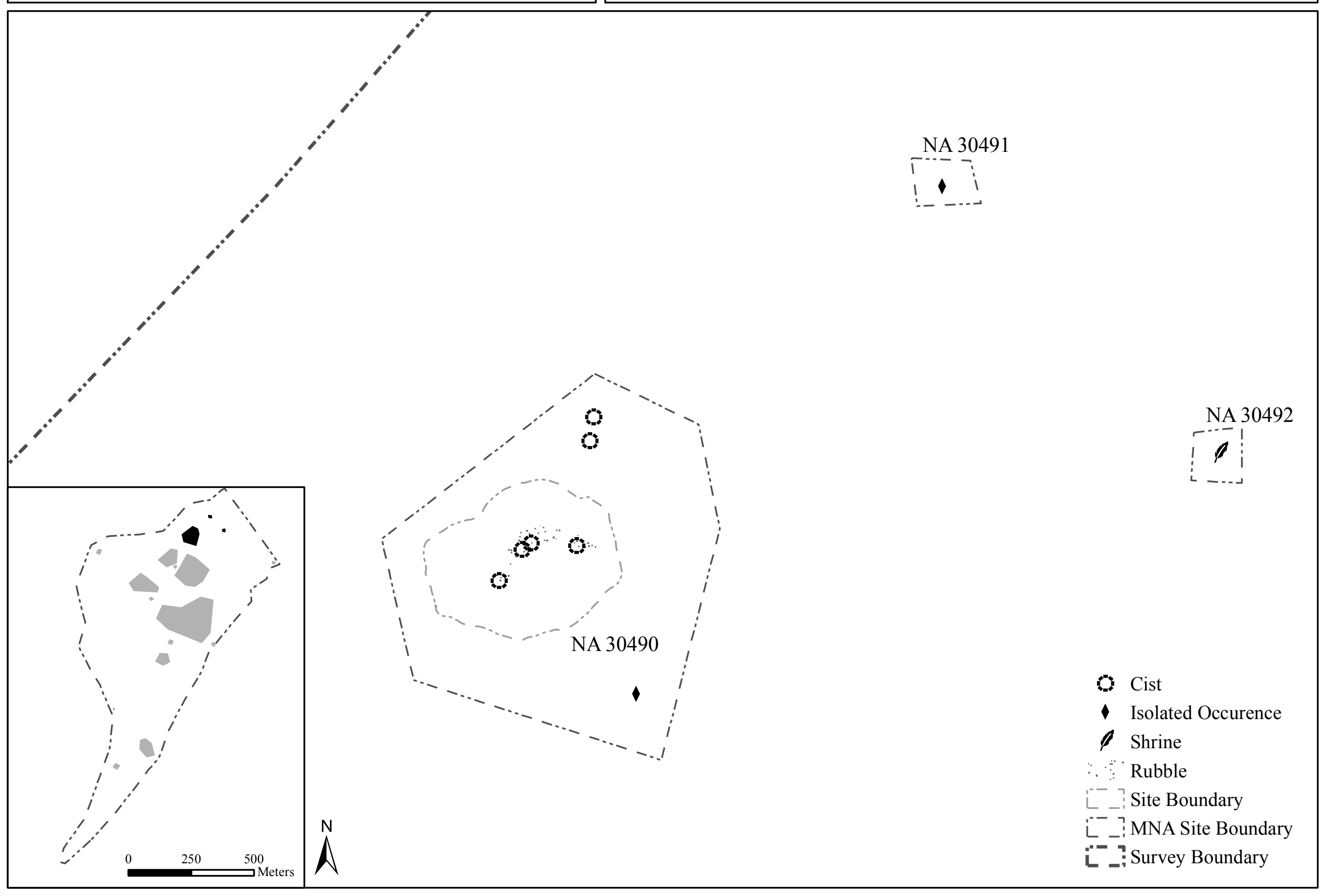




\section{First Mesa Survey \\ NA30481; NA30482; NA30483; NA30485; NA30486}

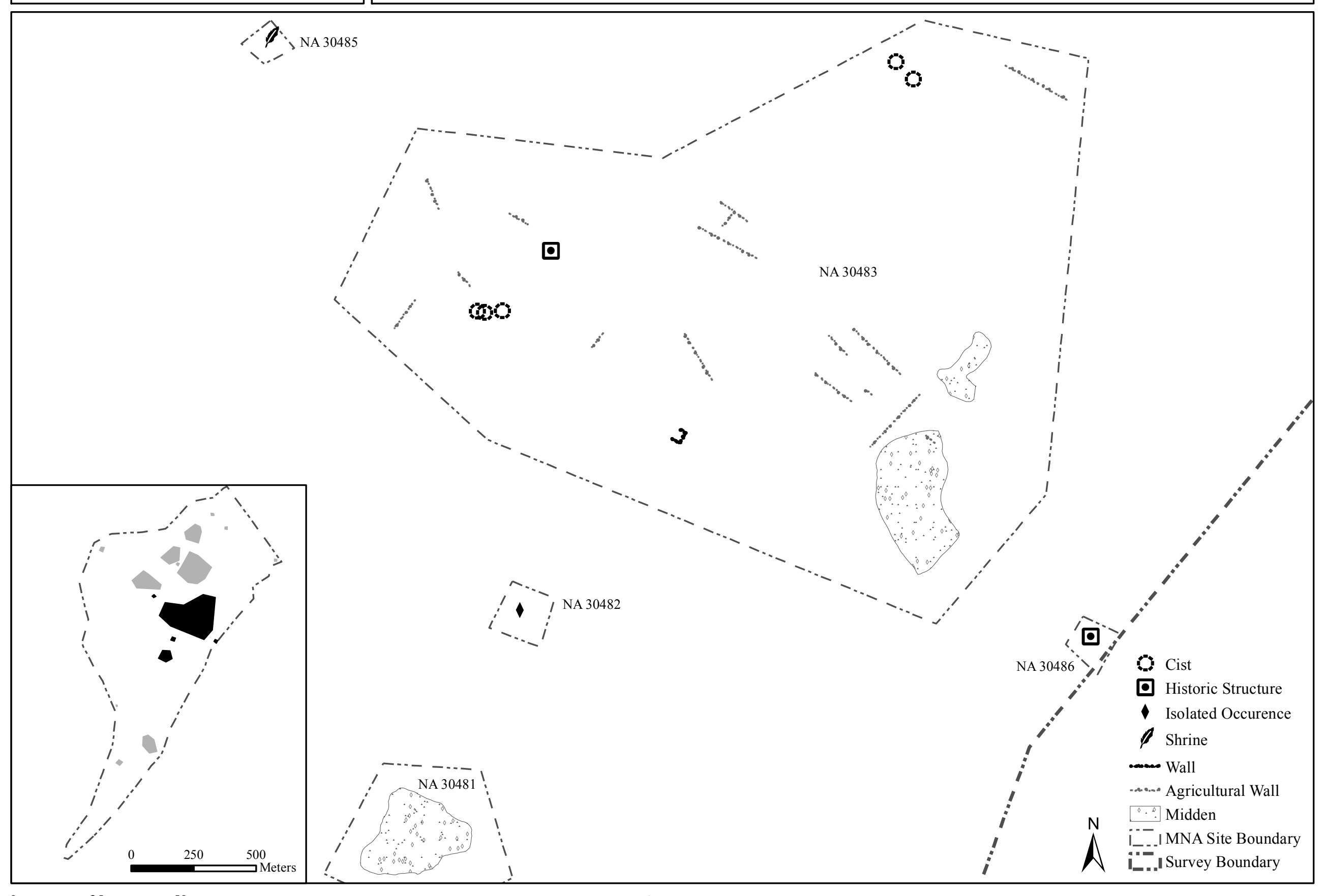




\section{First Mesa Survey}

NA30480; NA30494; NA30495

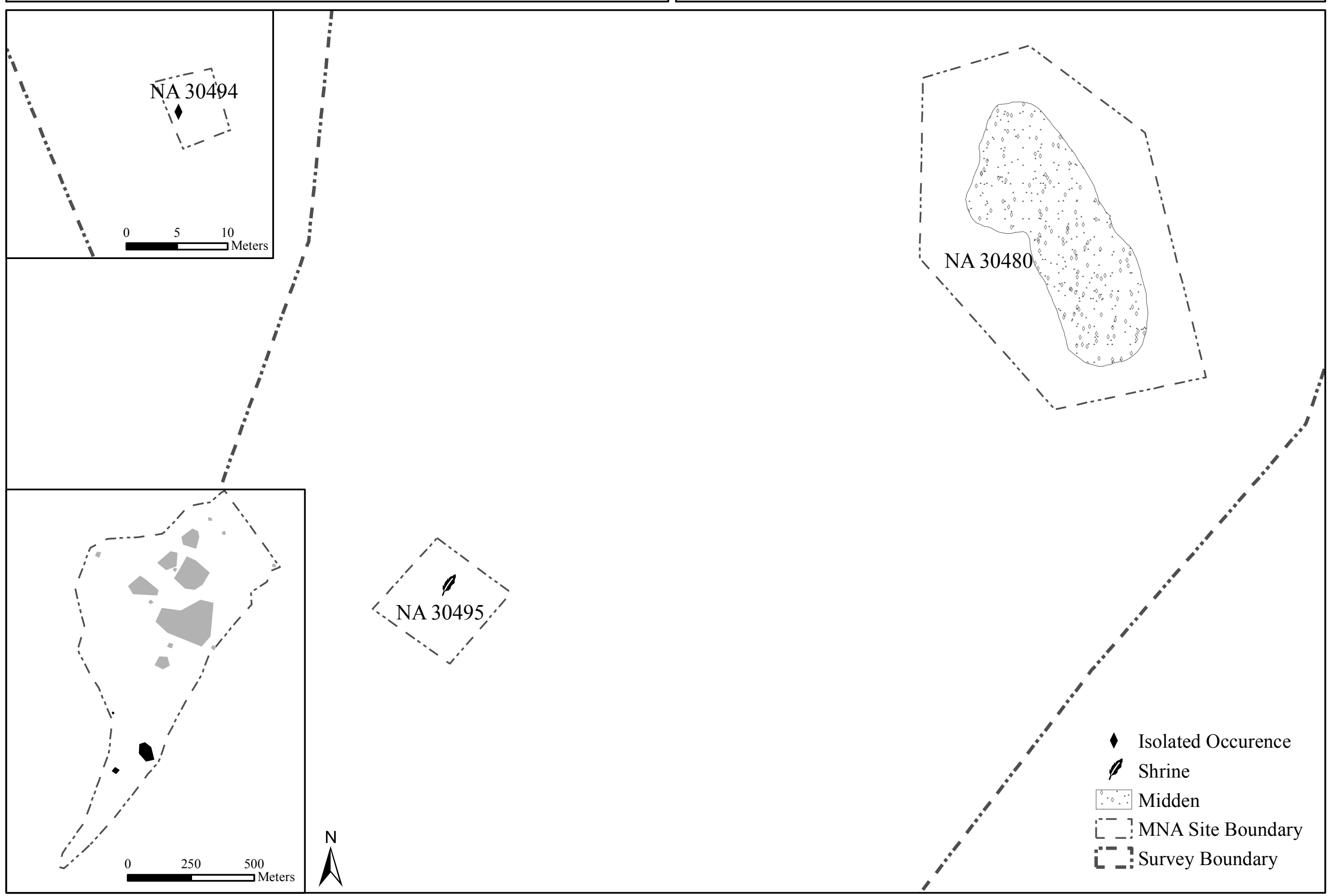

25 


\section{First Mesa Survey}

NA30487; NA30484; NA30485

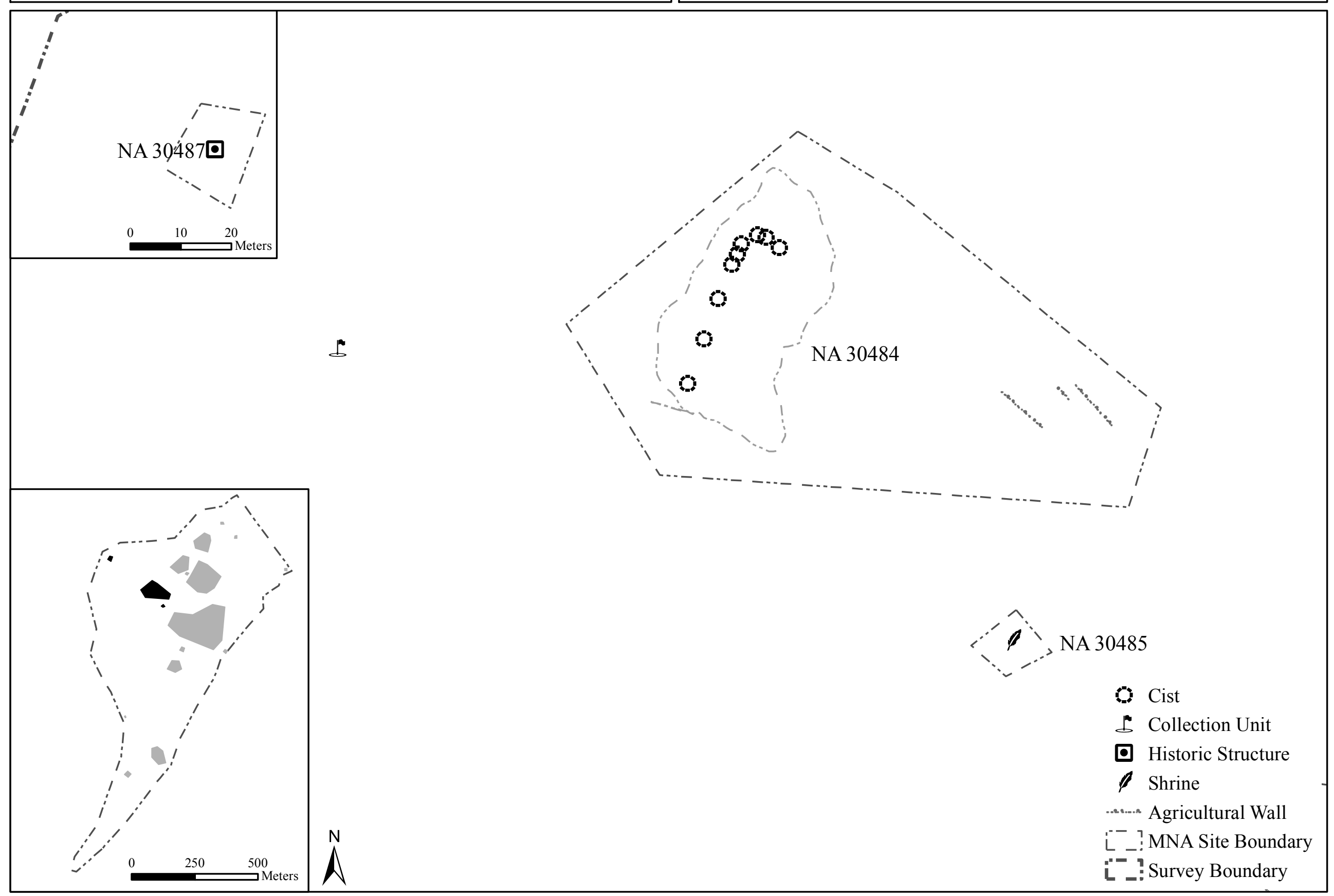




\section{Giant Chair}

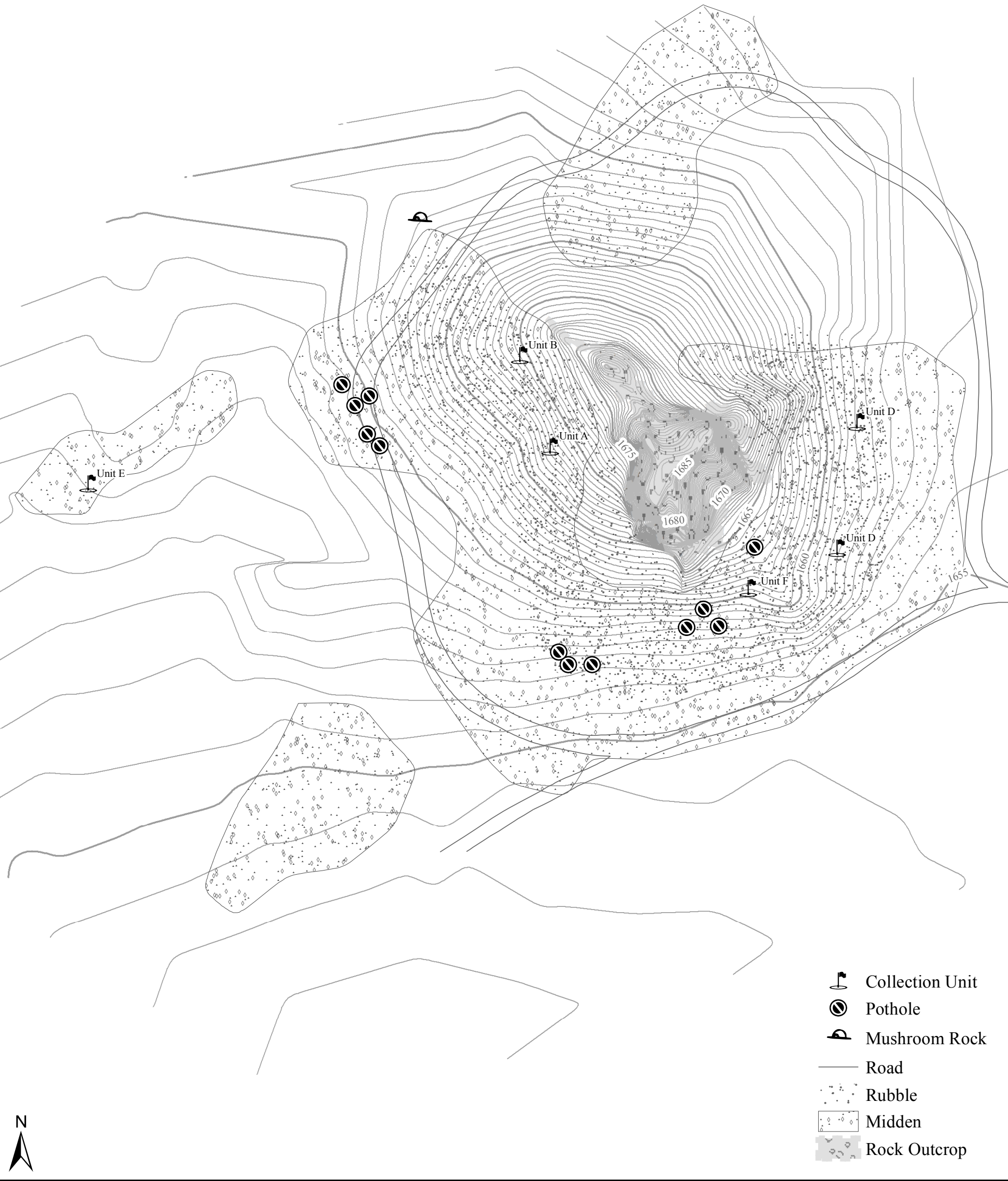




\section{Hukovi}

NA844

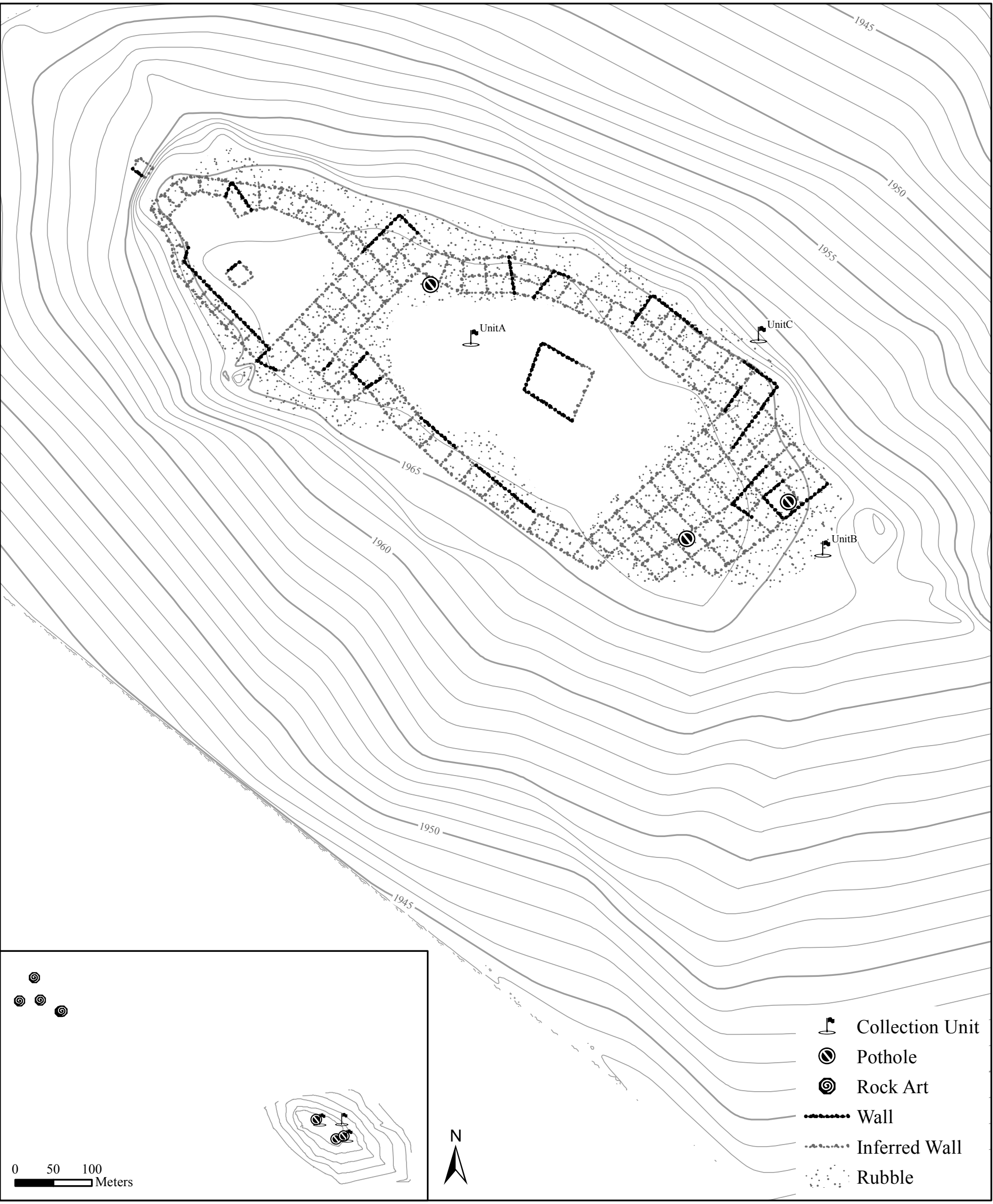




\section{Huvatuqwa \\ NA3095; NA30665; NA30666}

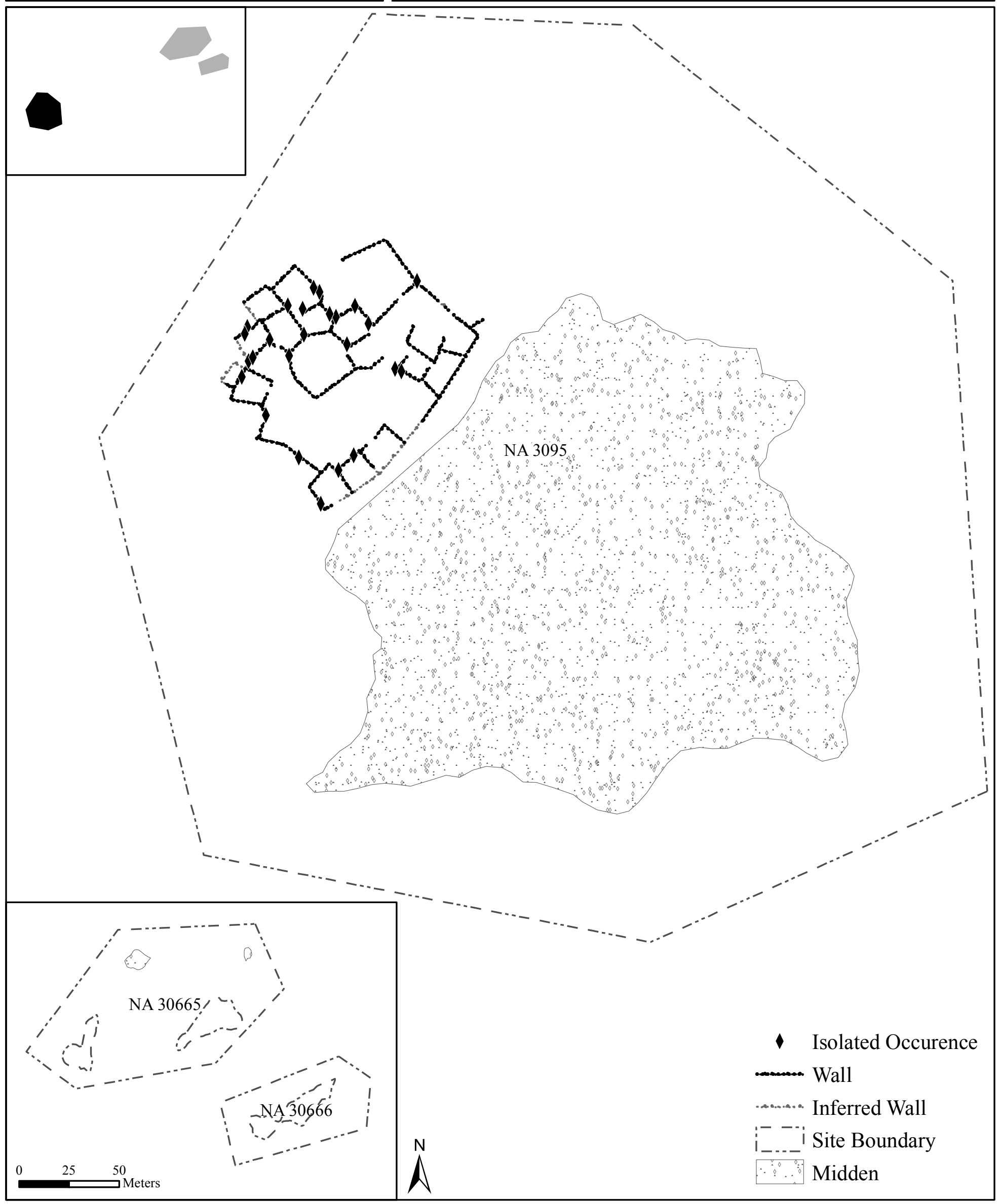




\section{Kawayka'a}

NA1001

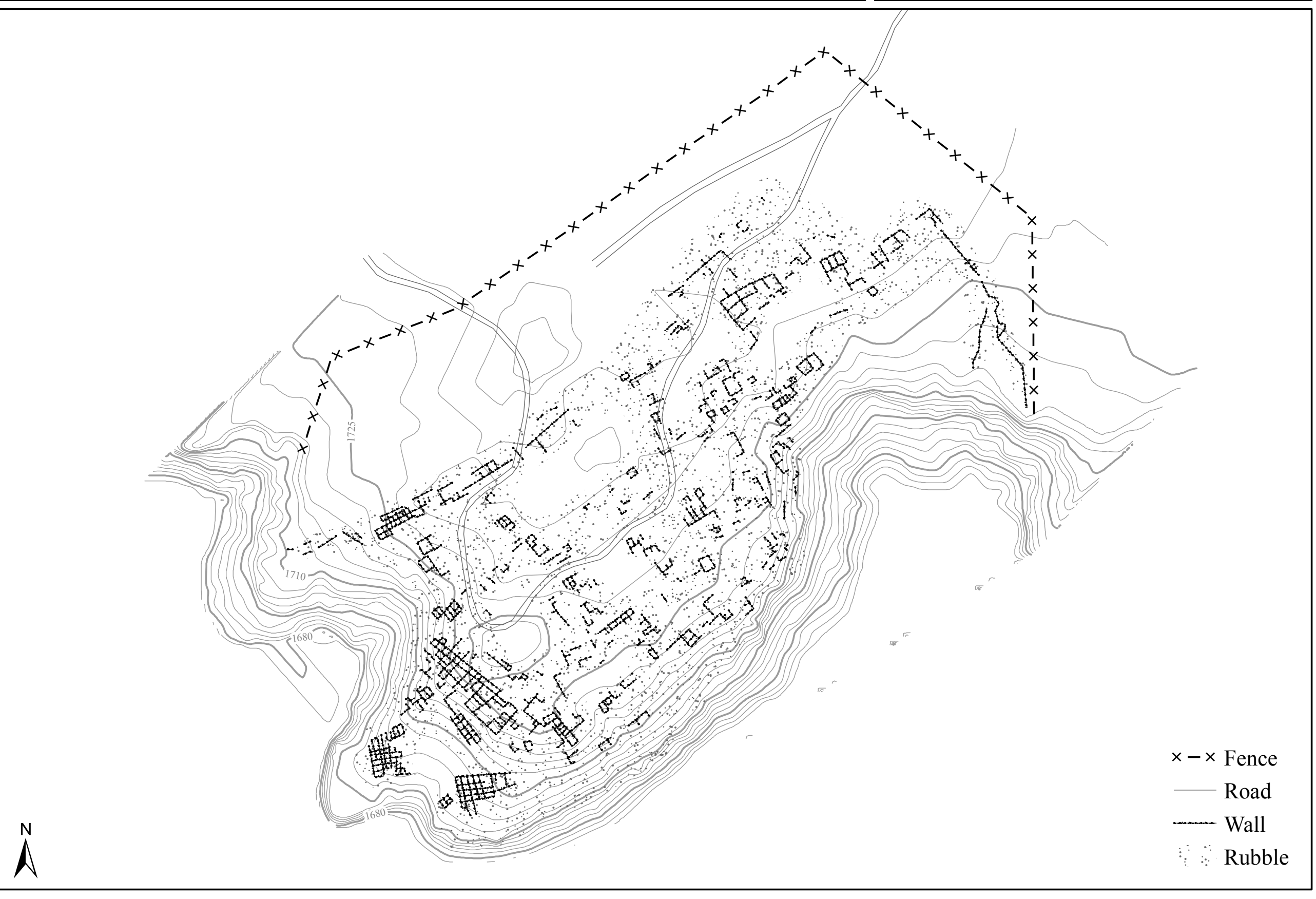




\section{Kisak'ovi}

NA813

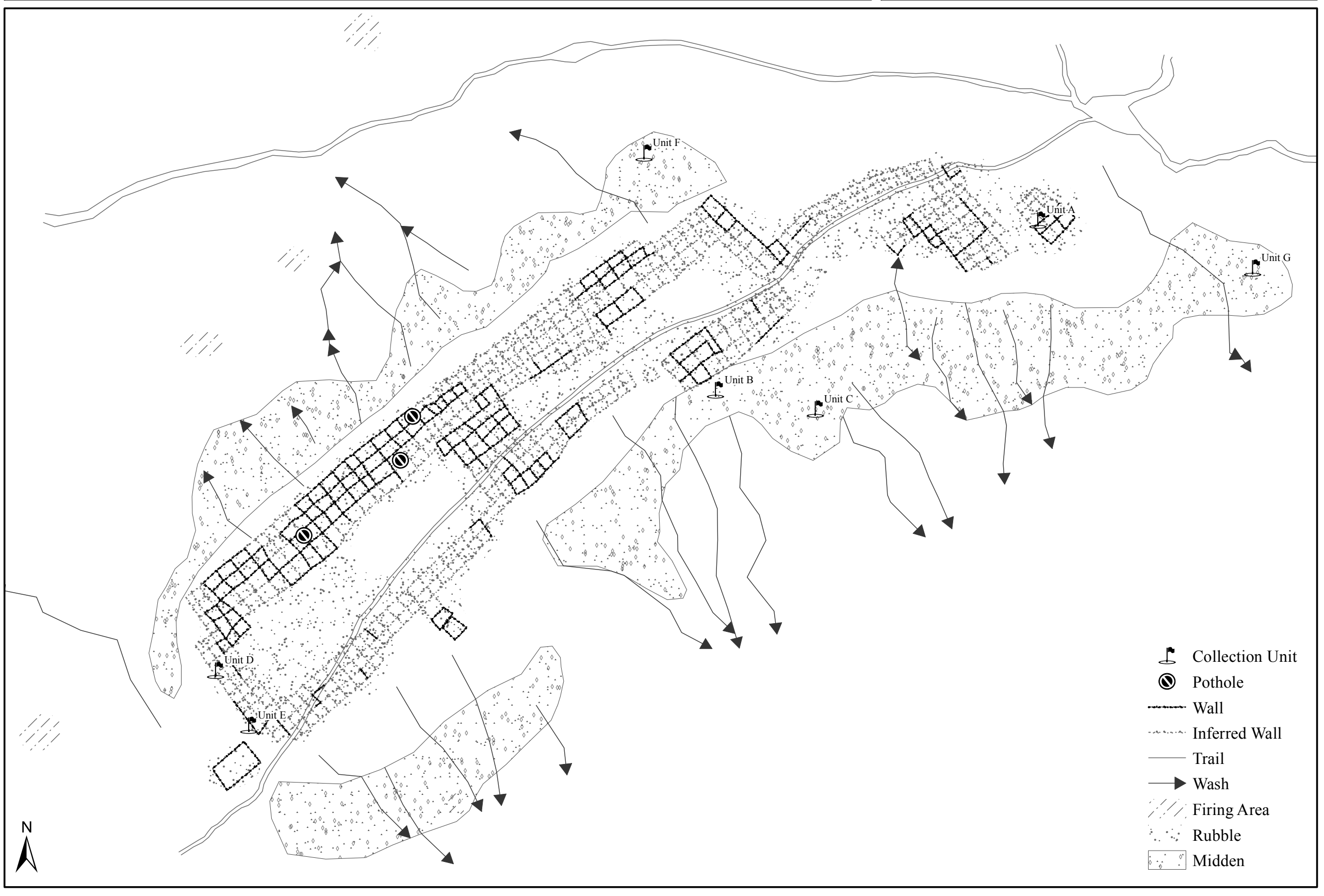




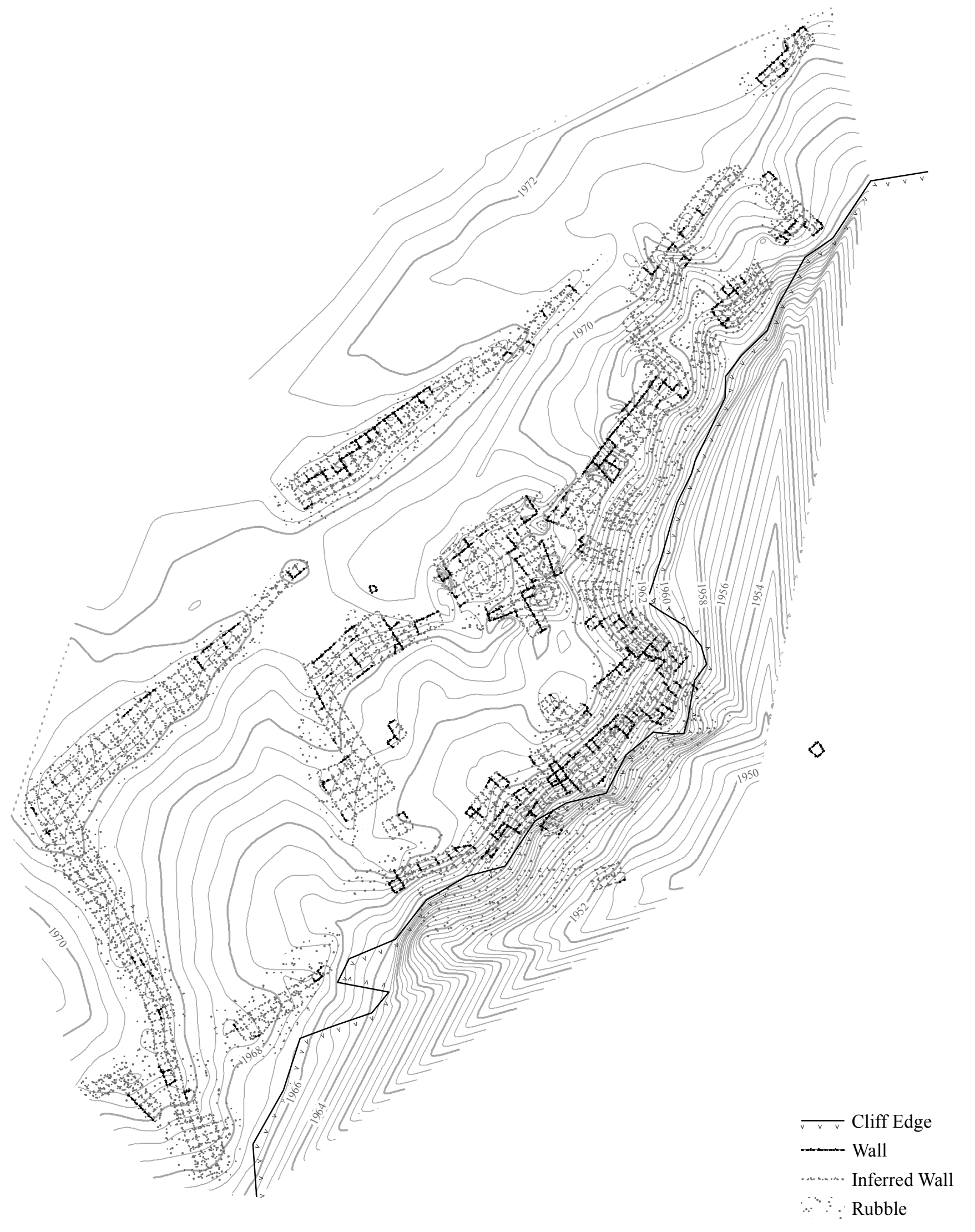

i 


\section{Kokotsomo}

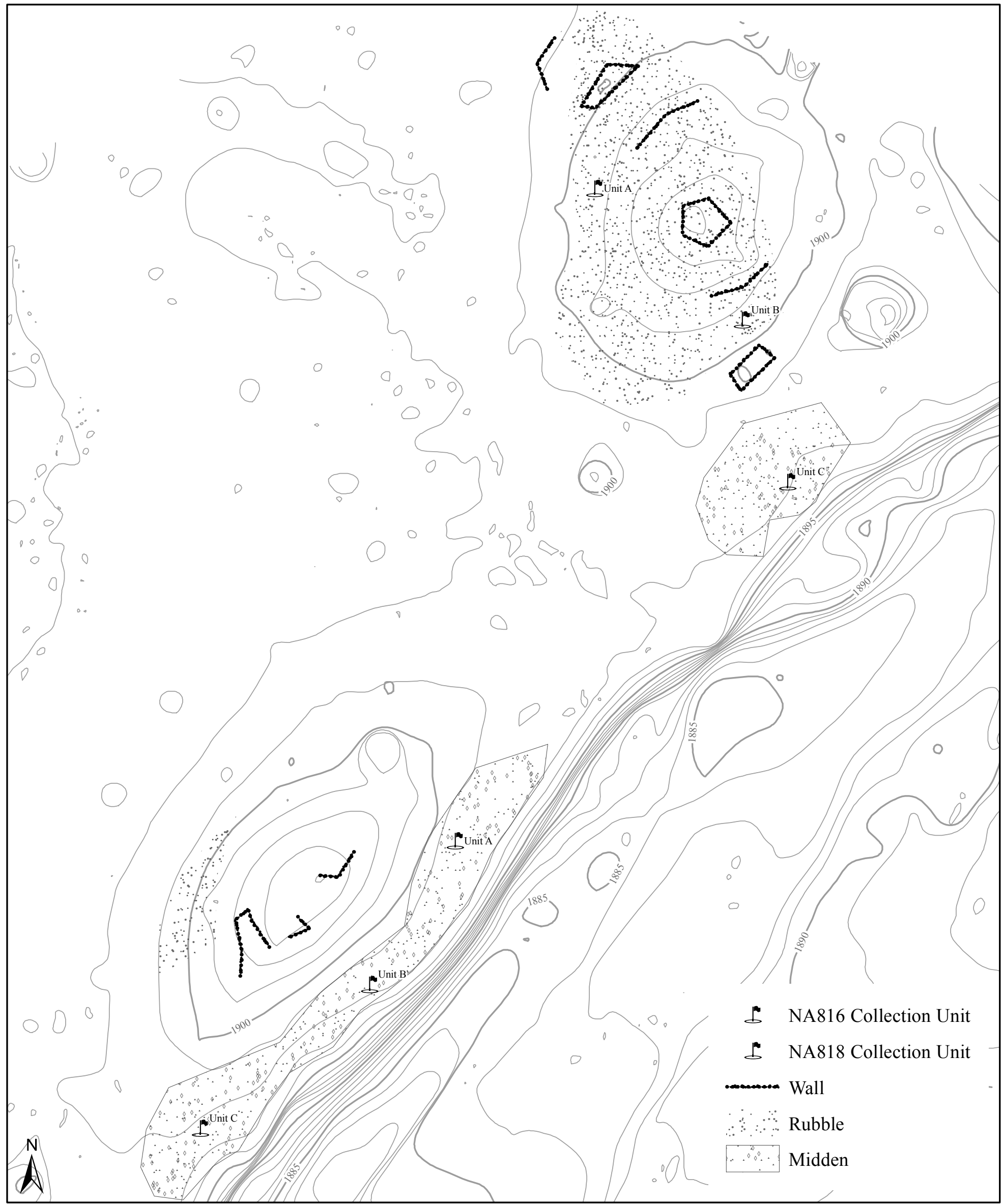




\section{Lemeva \\ NA1707}

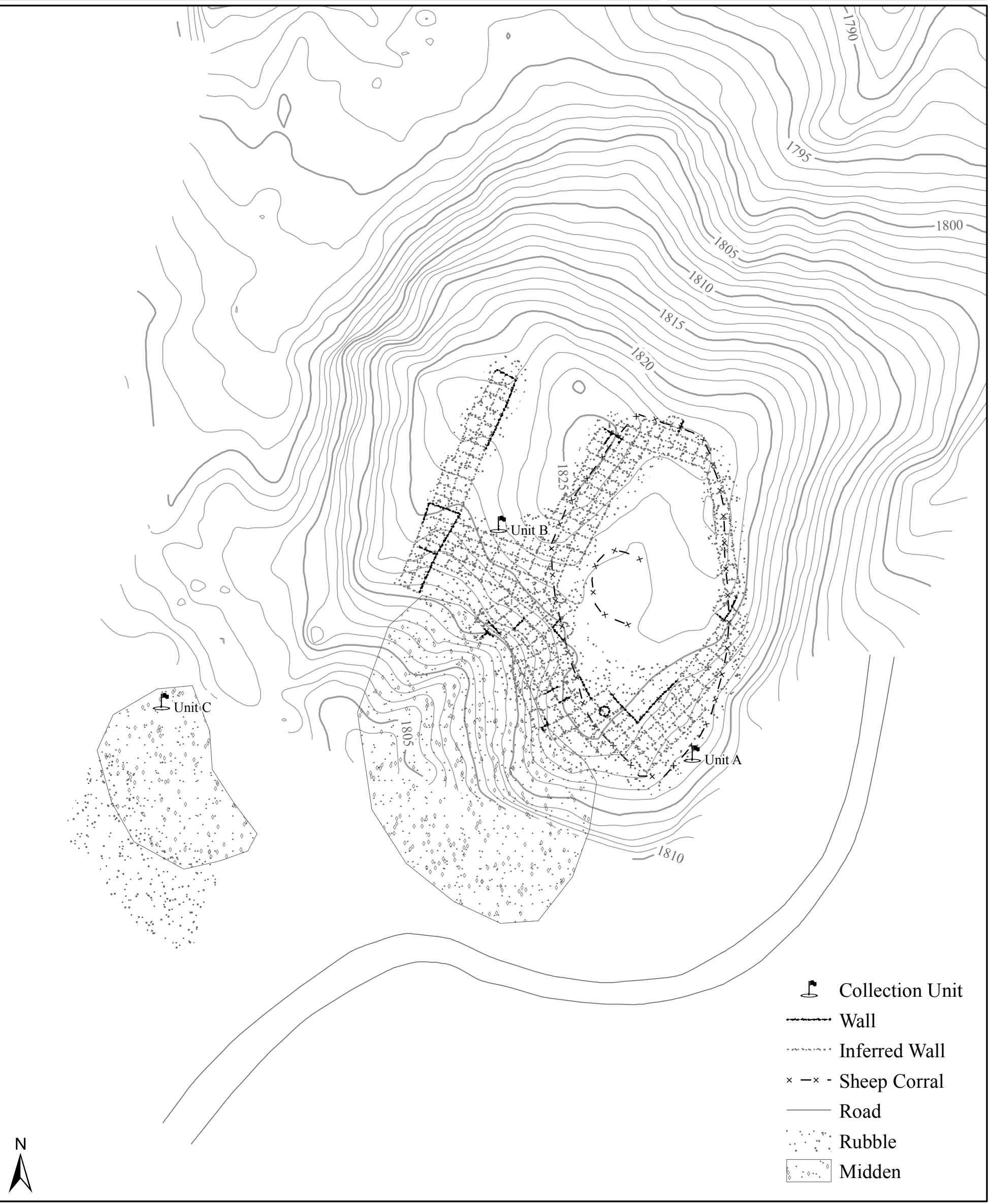




\section{Lolkangtuuyqa \\ NA1049}

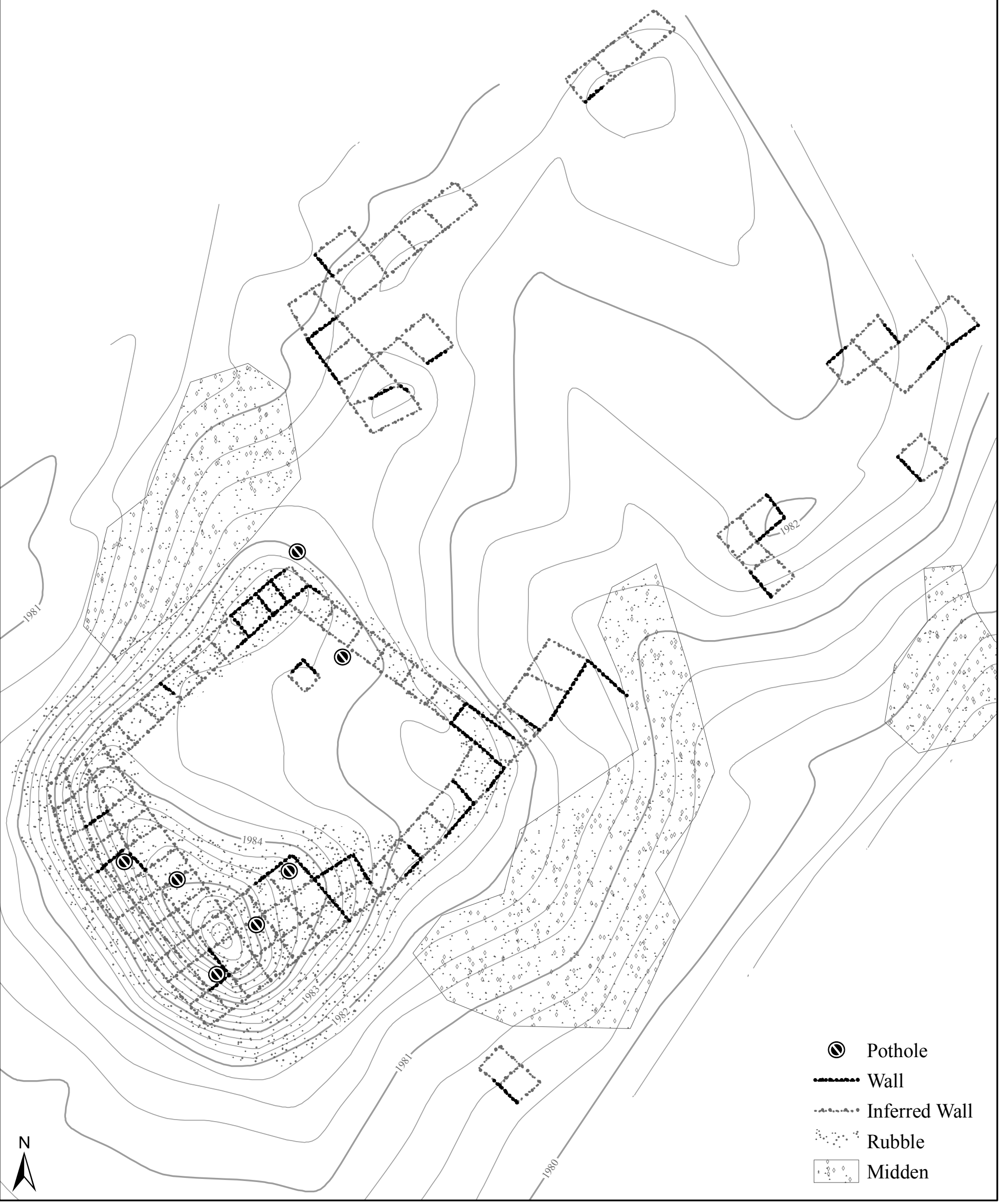




\section{Mosiptanga}

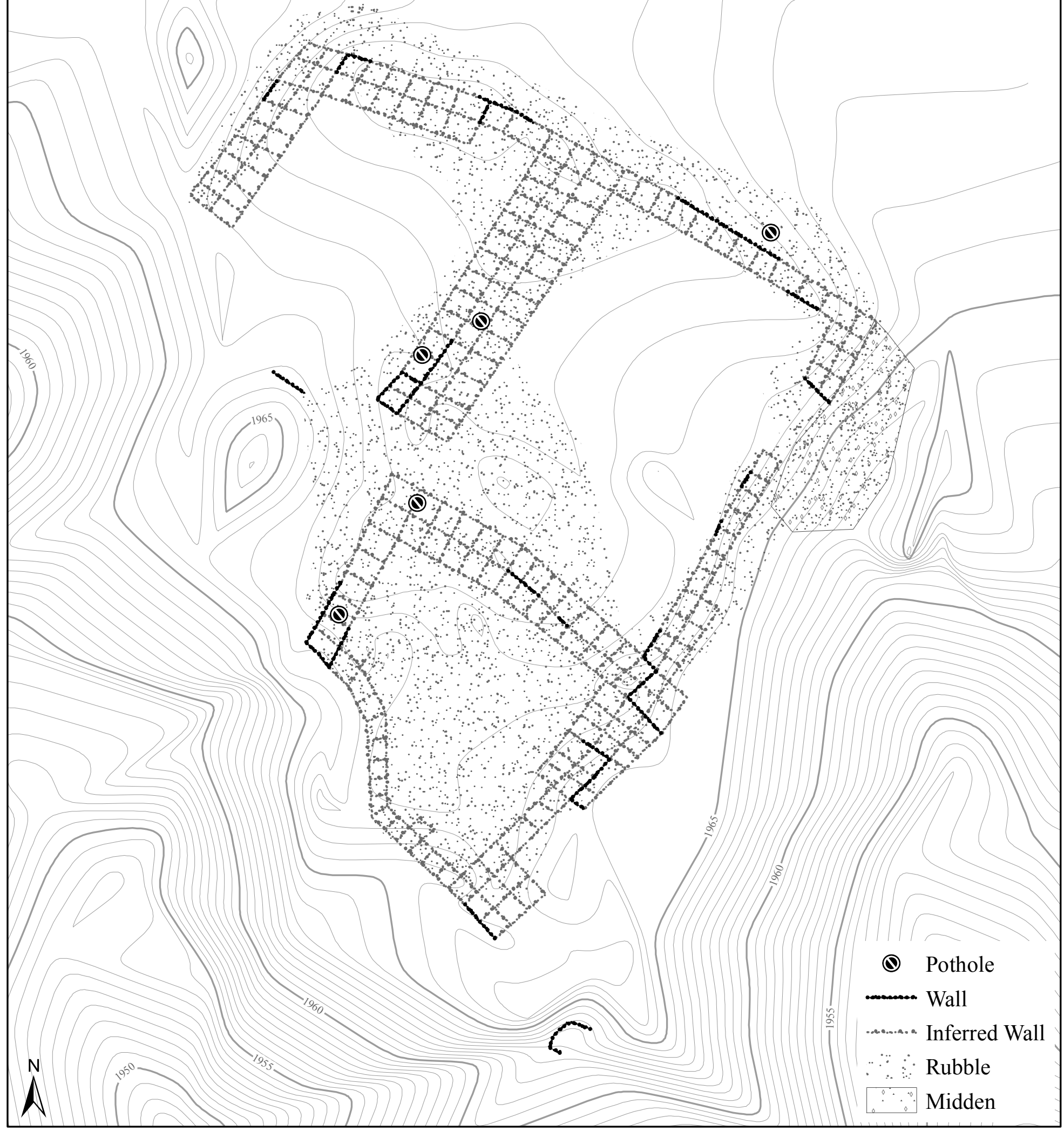




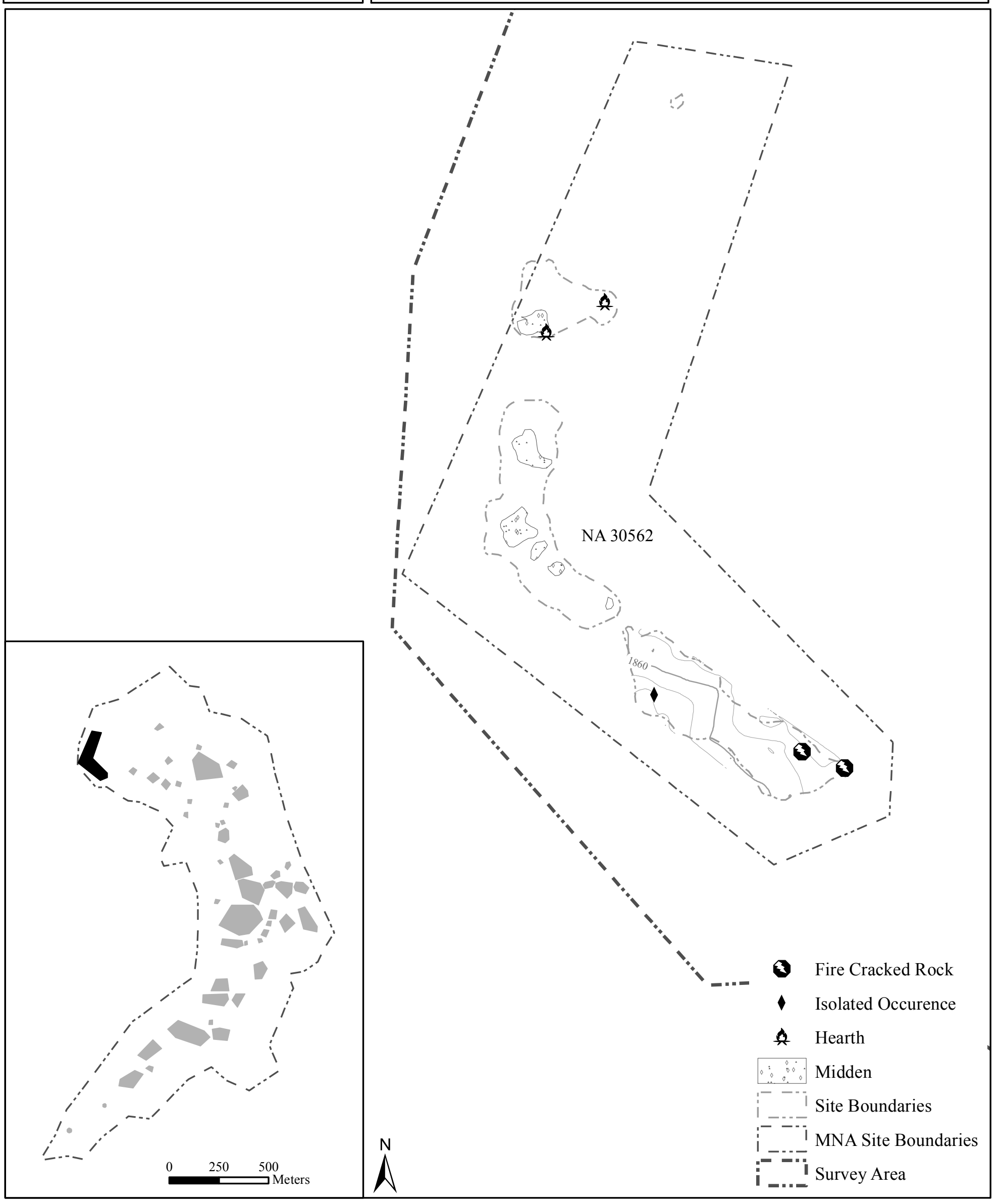




\section{Mungyaovi Survey}

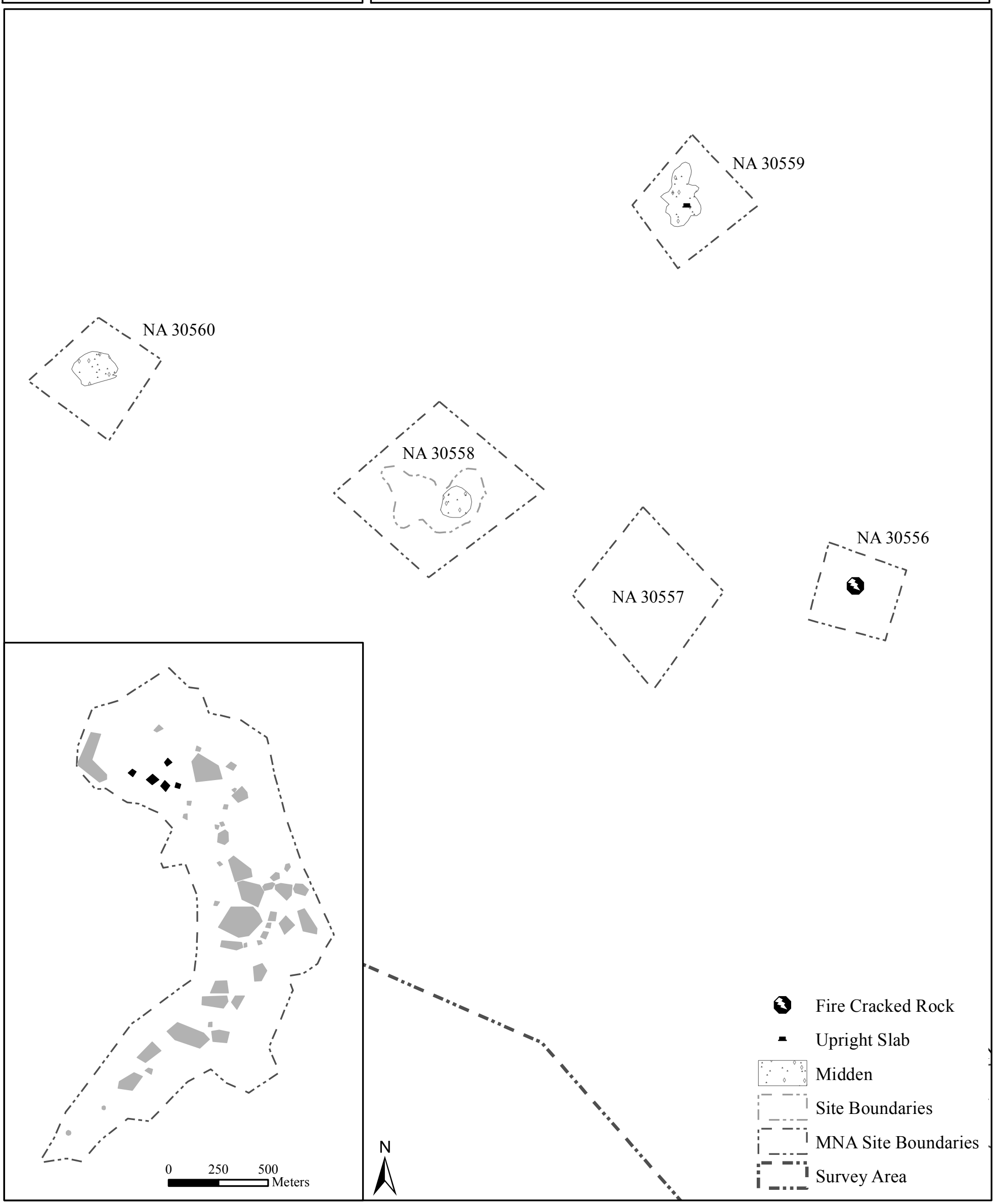




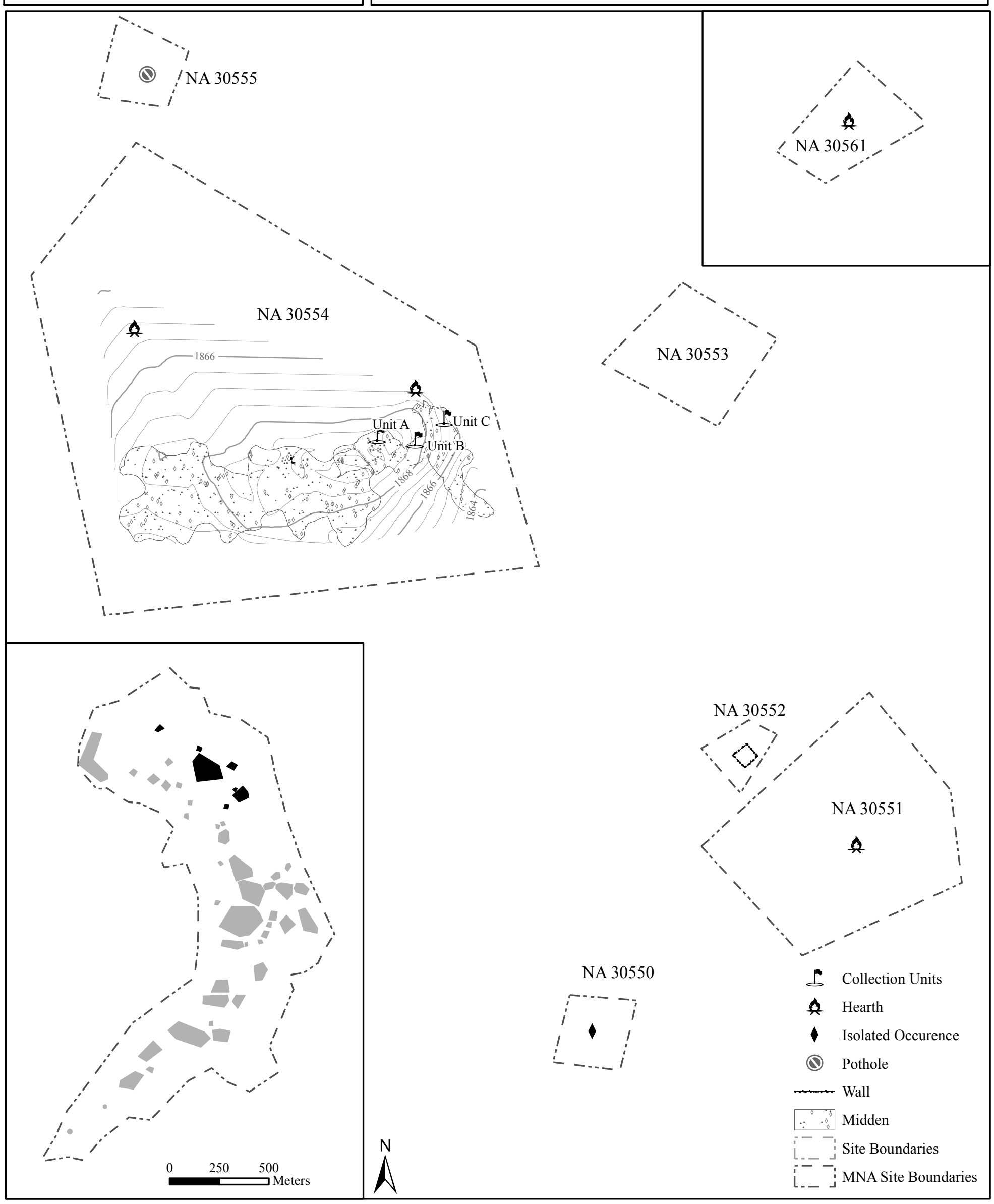




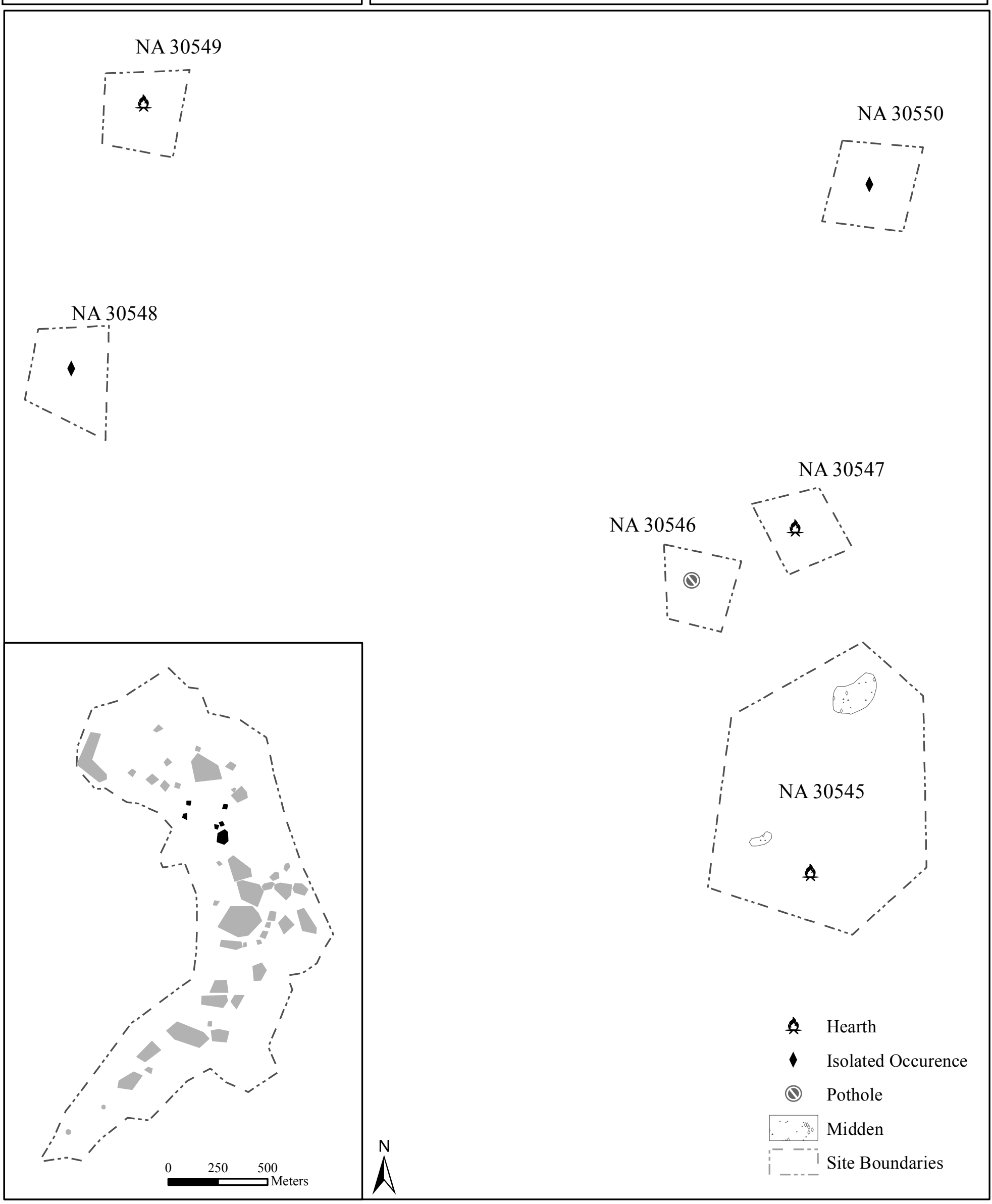




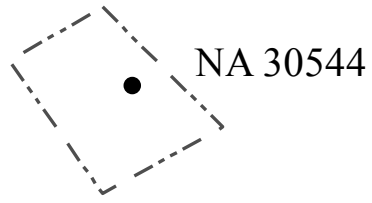

Cist

\& Collection Units

3 Fire Cracked Rock

f Hearth

- Upright Slab

- Clay Source

Fire Cracked Rock Area

$\therefore \therefore 0^{\circ}$ Midden

Site Boundaries
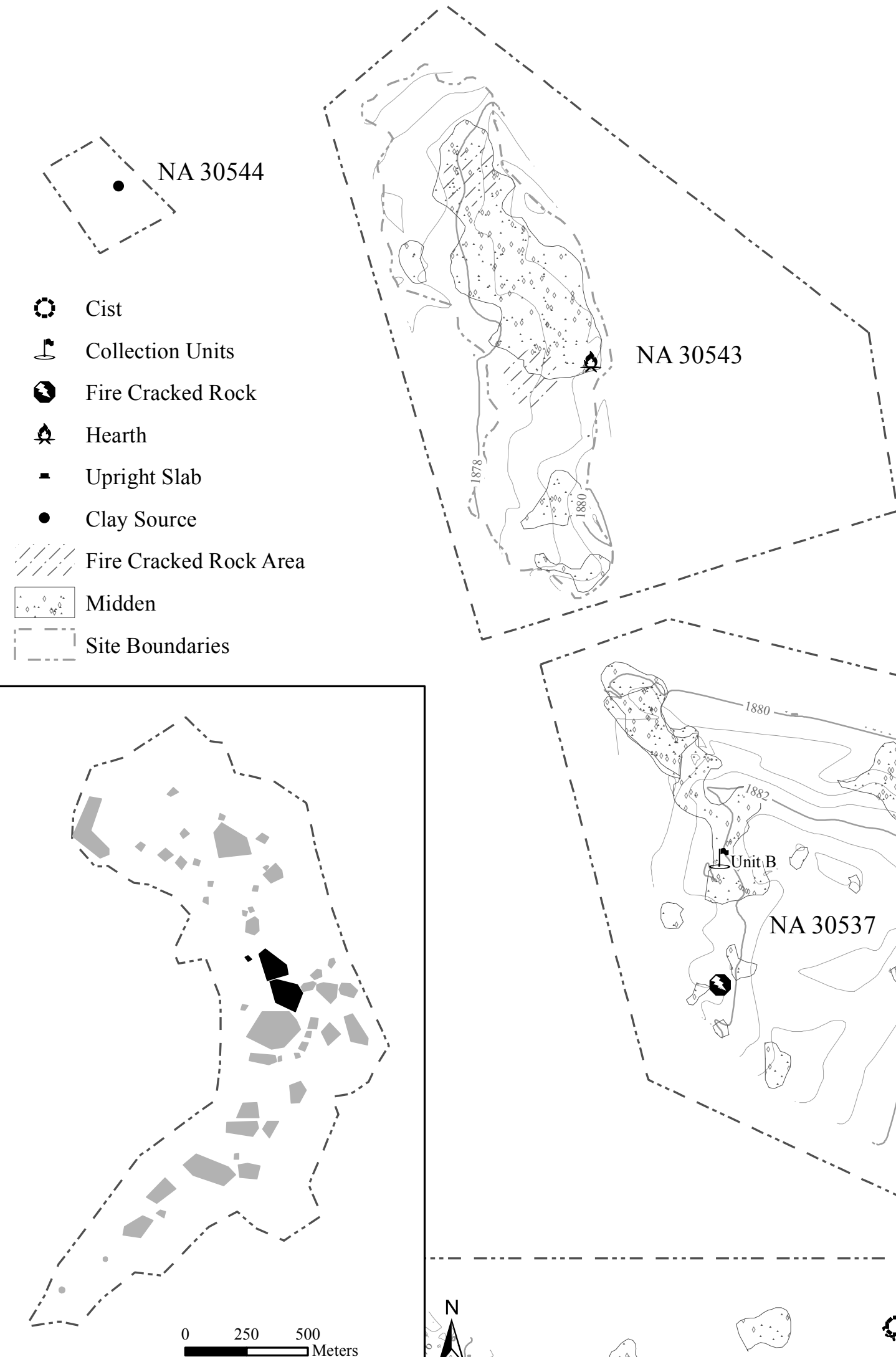

Meters

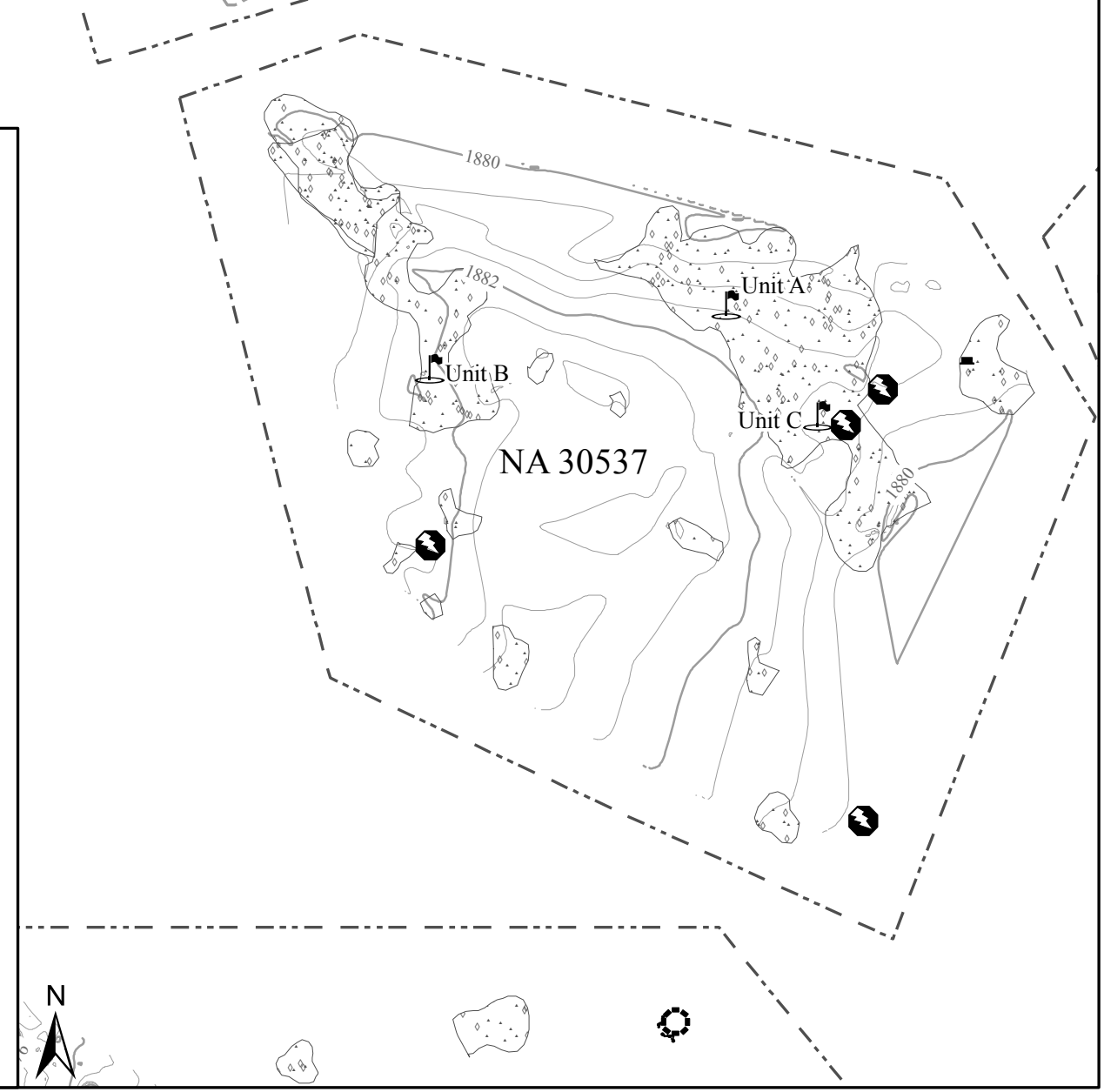




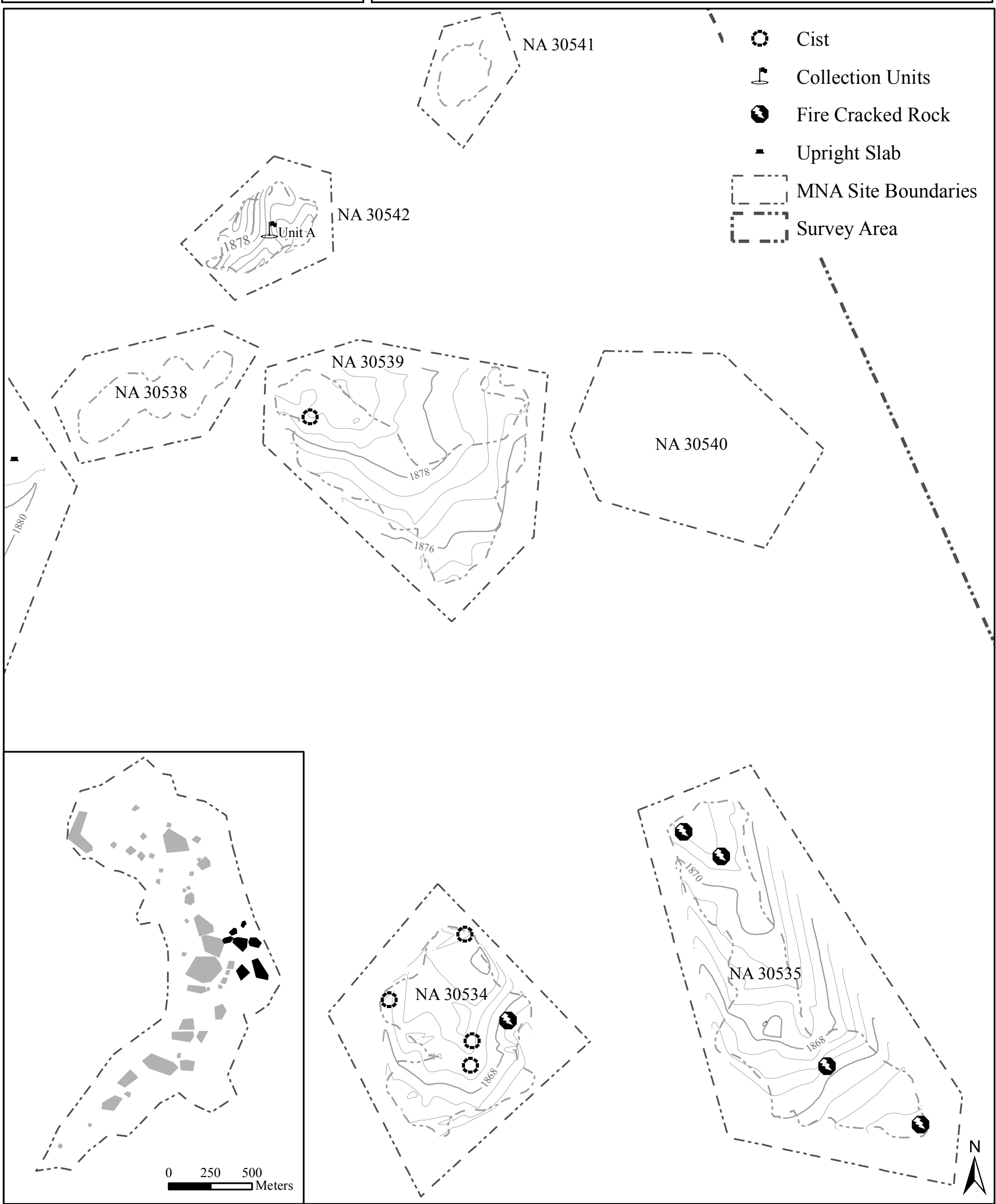




\section{Mungyaovi Survey}

\section{NA30526 - NA30533}

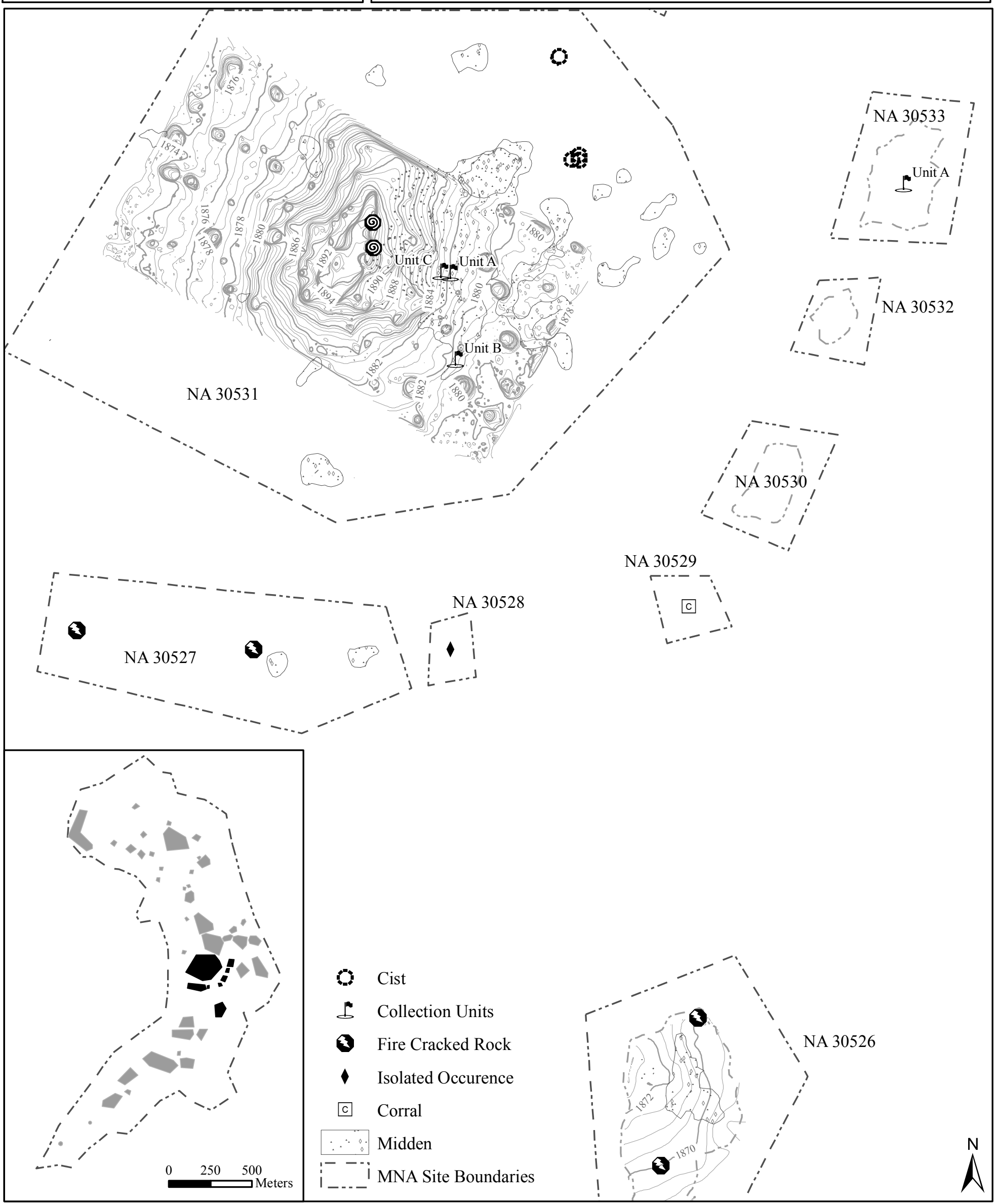




\section{Mungyaovi Survey}

\section{NA30520 - NA30525}

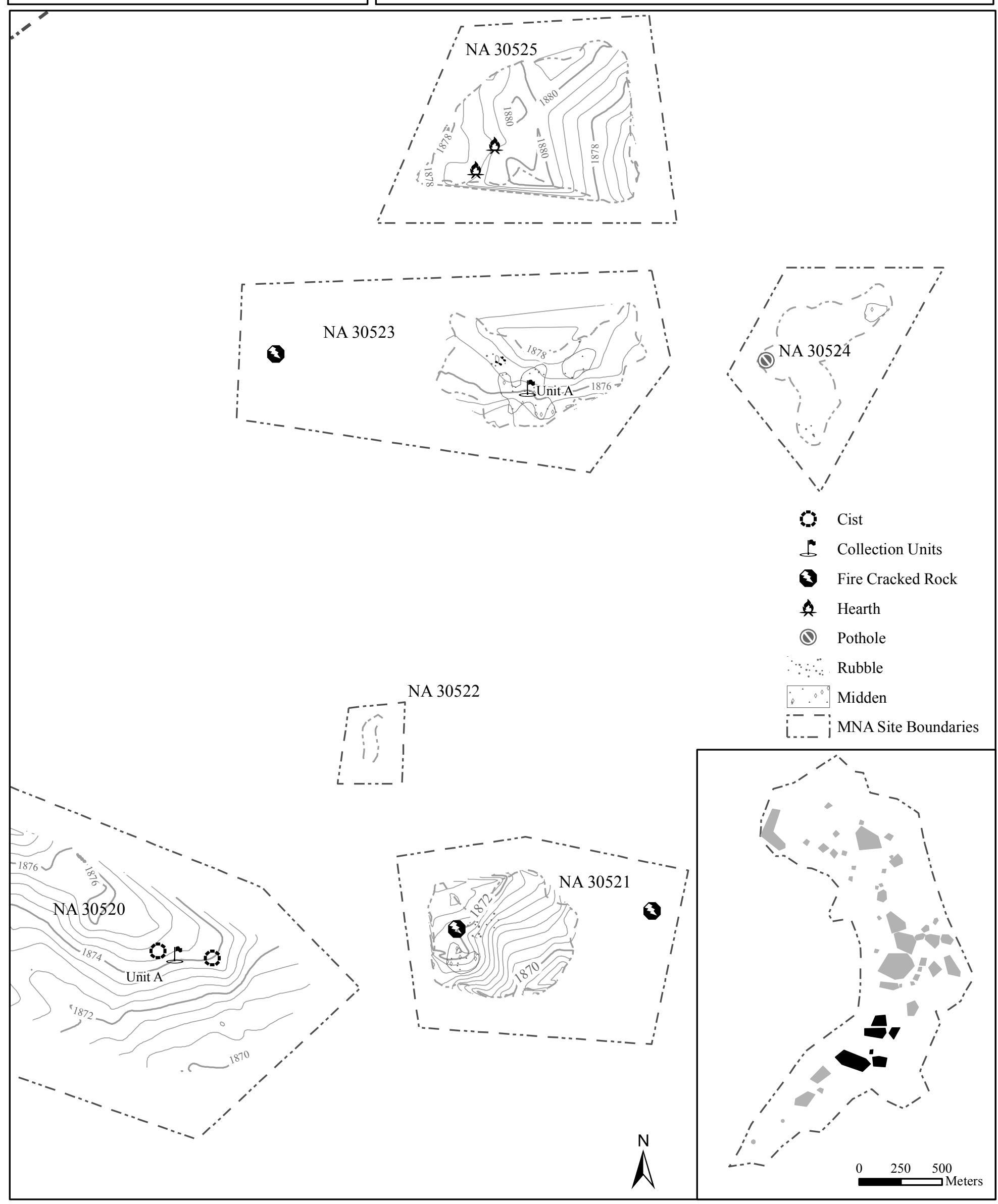




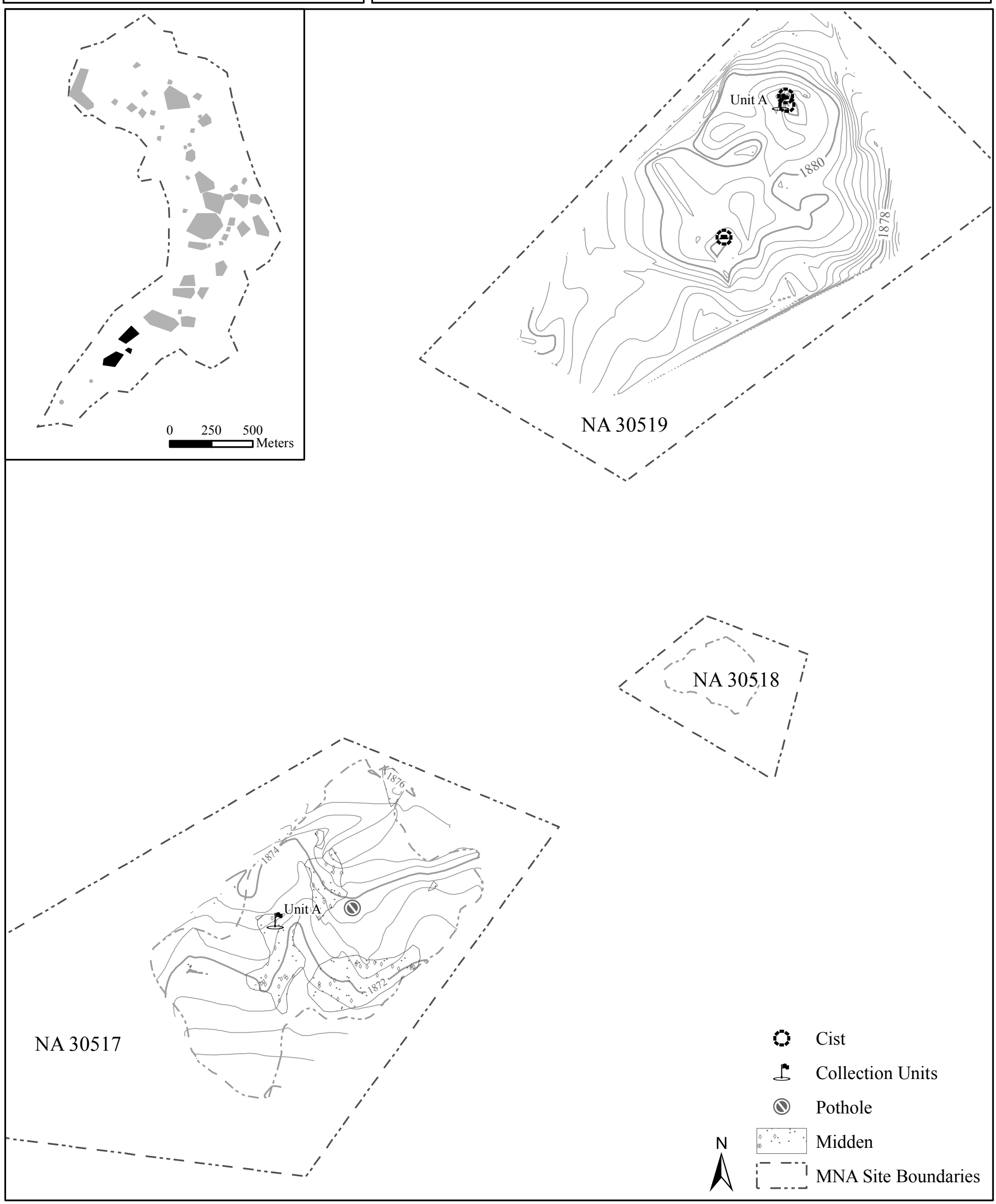




\section{Nakya'ovi}

NA28333

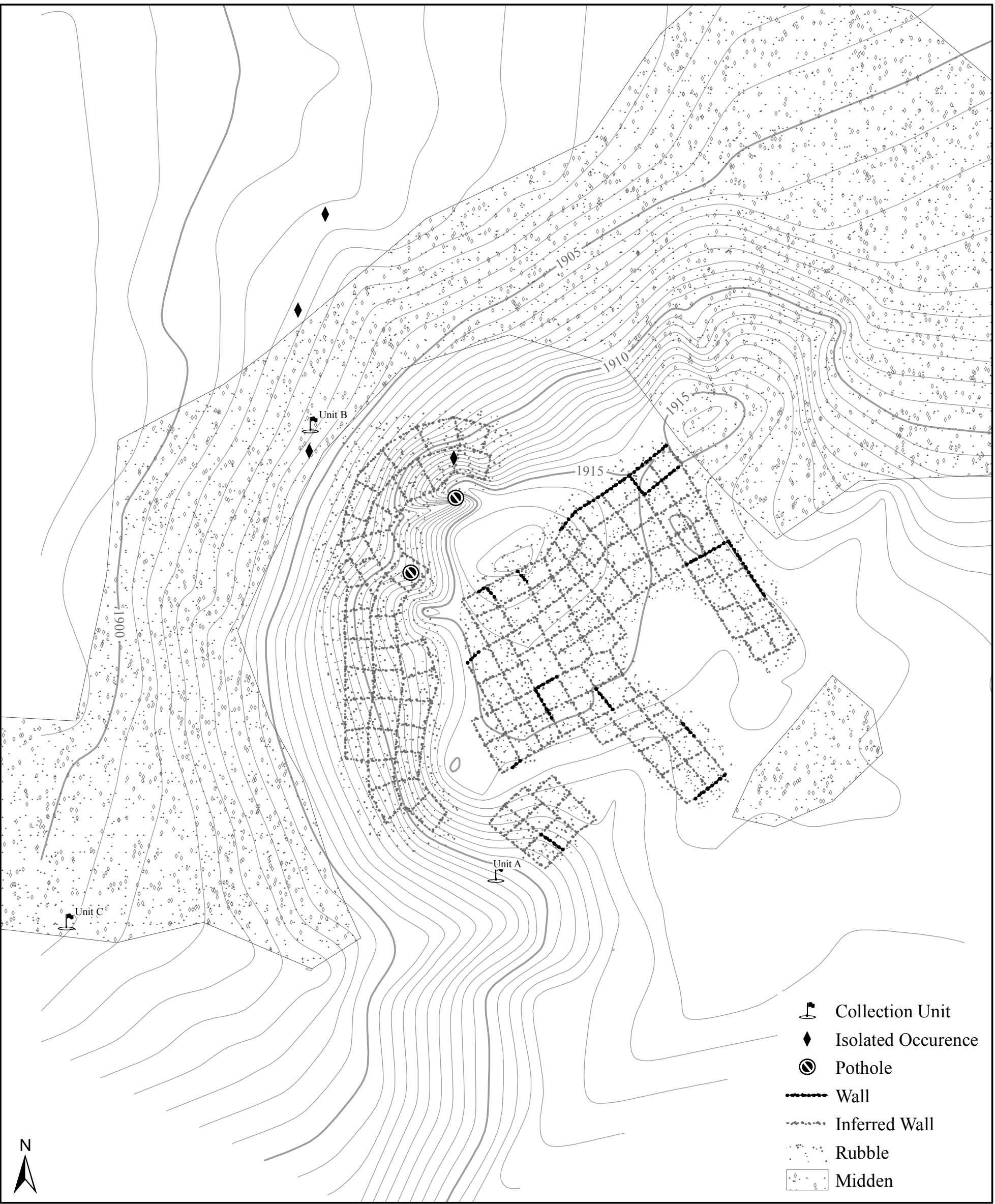




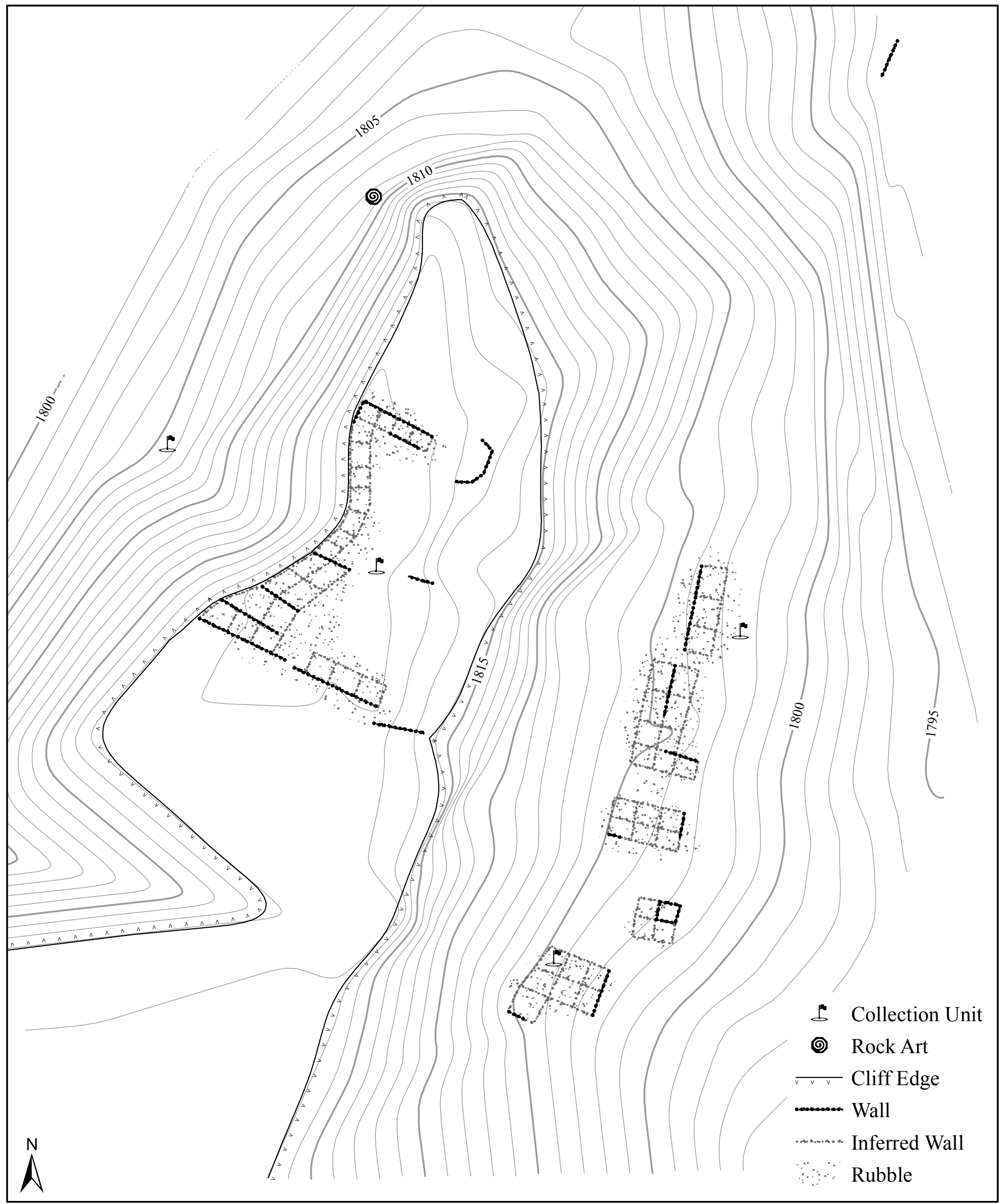




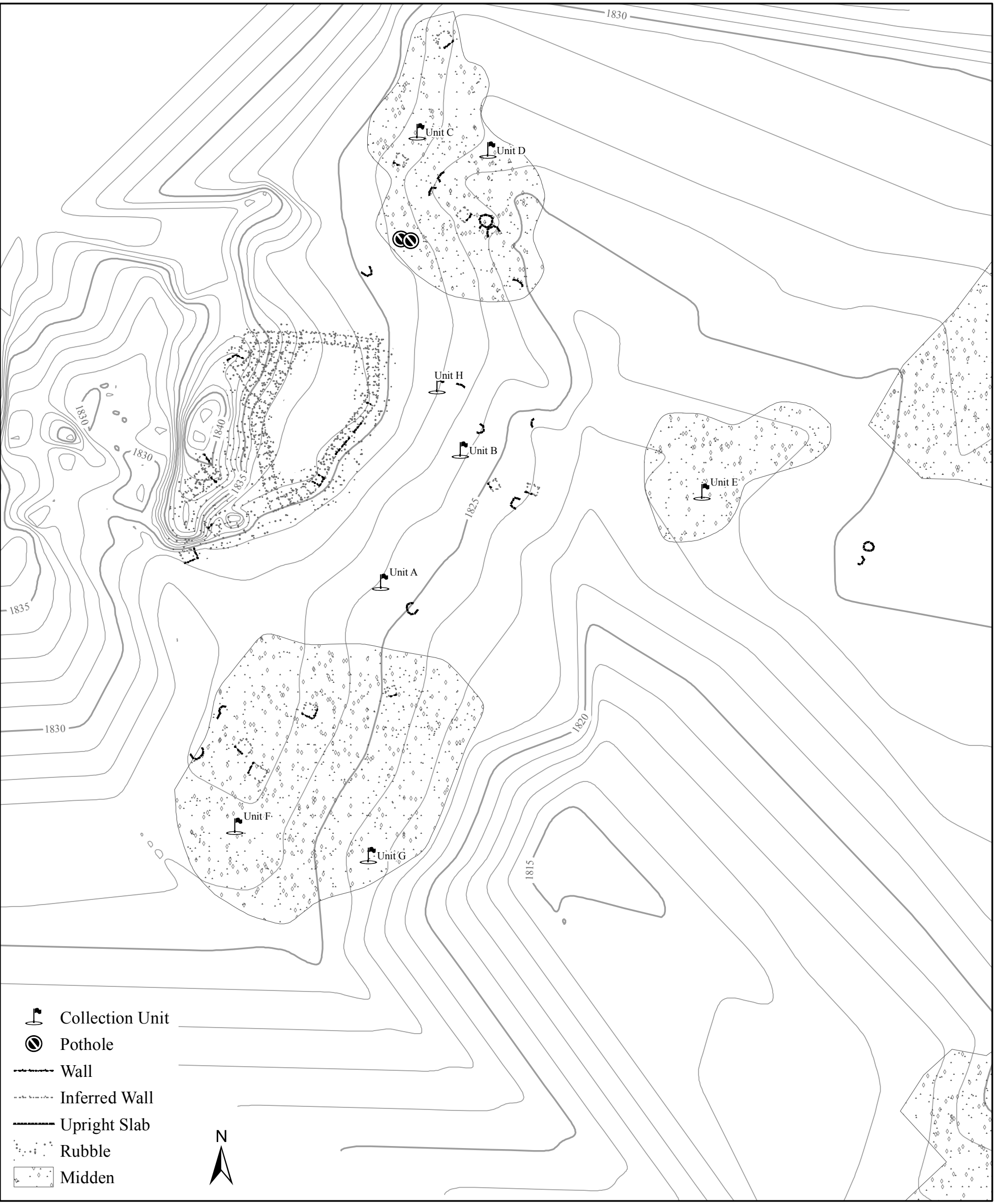




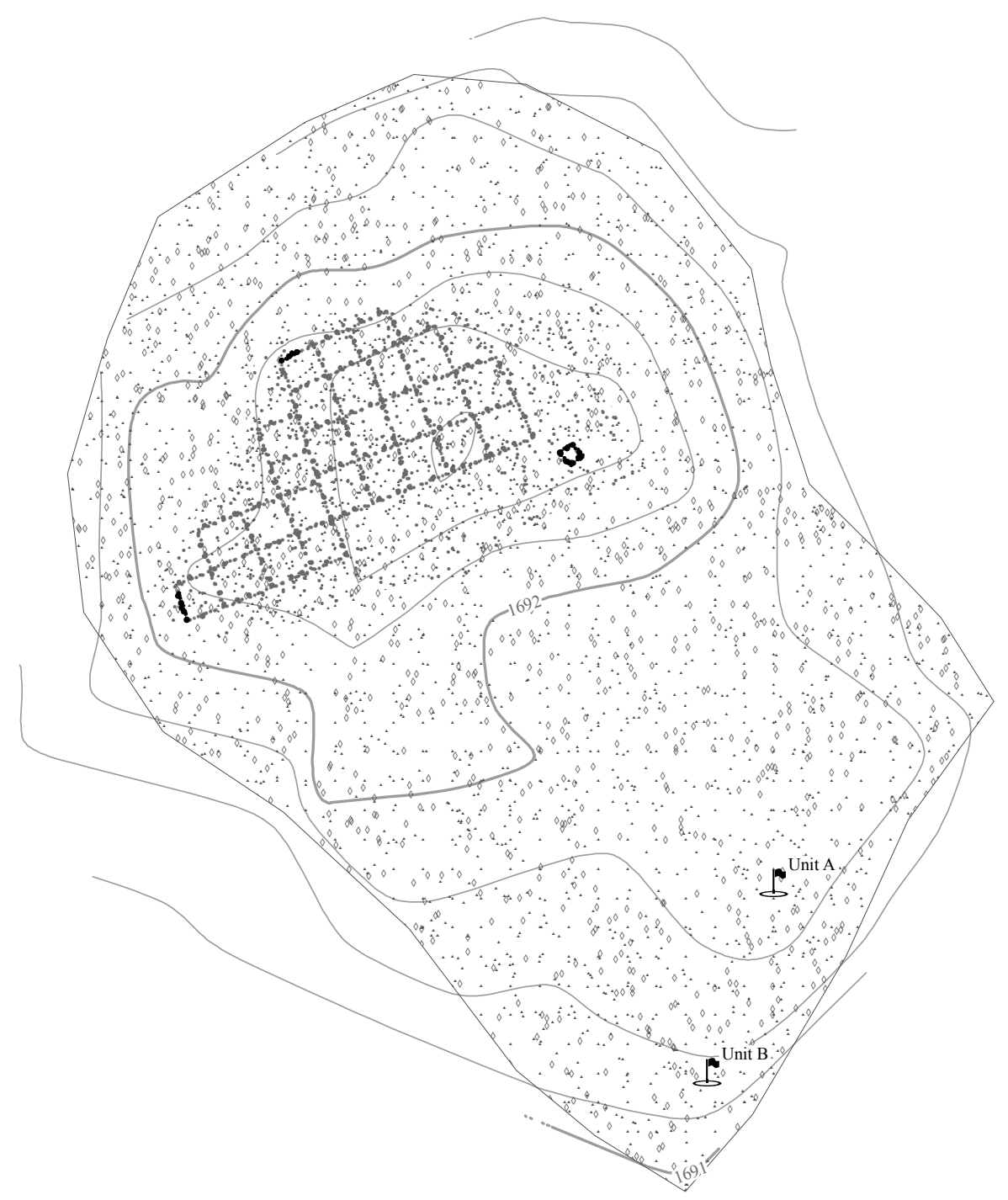

\& Collection Unit 


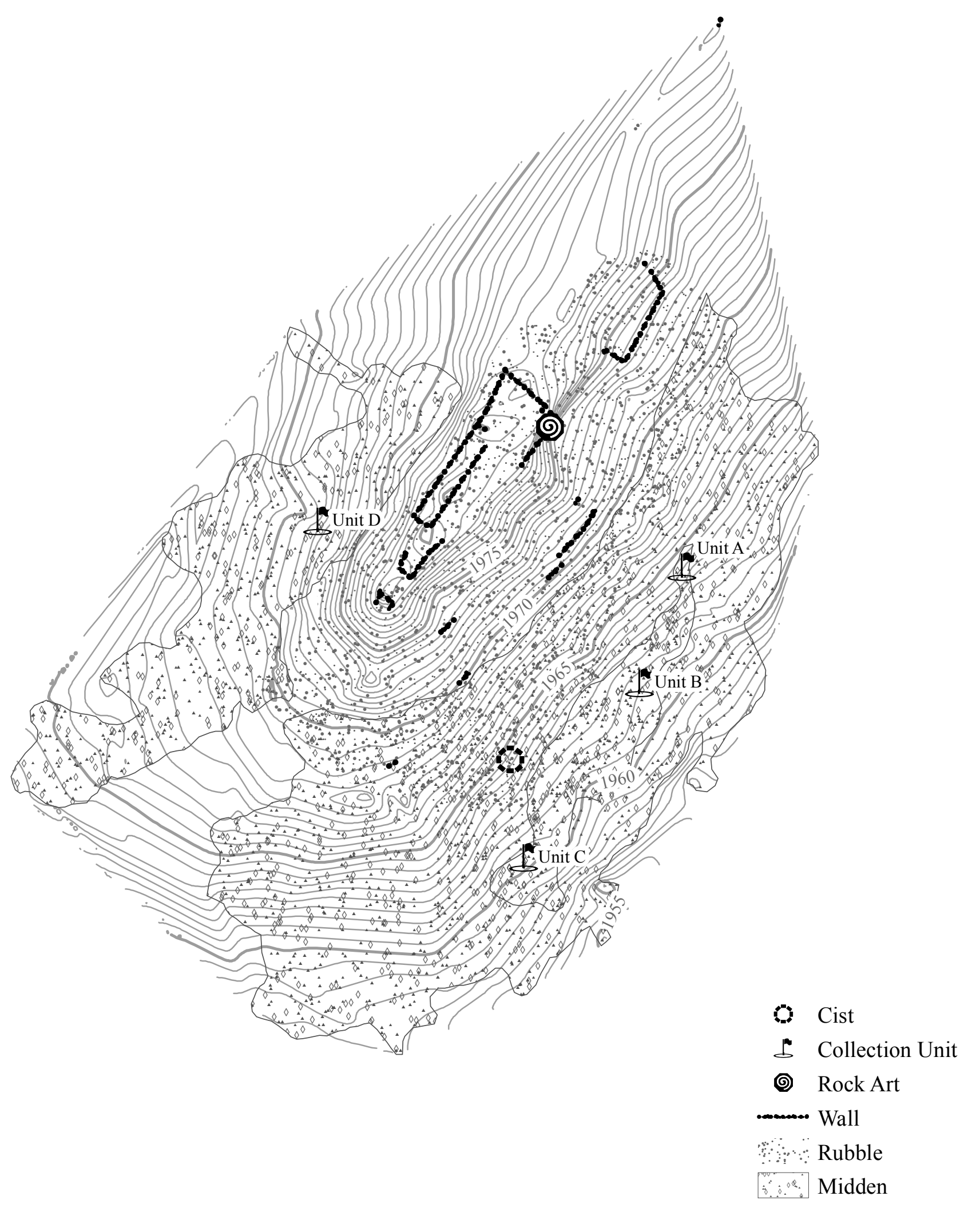




\section{Payupki}

NA1040

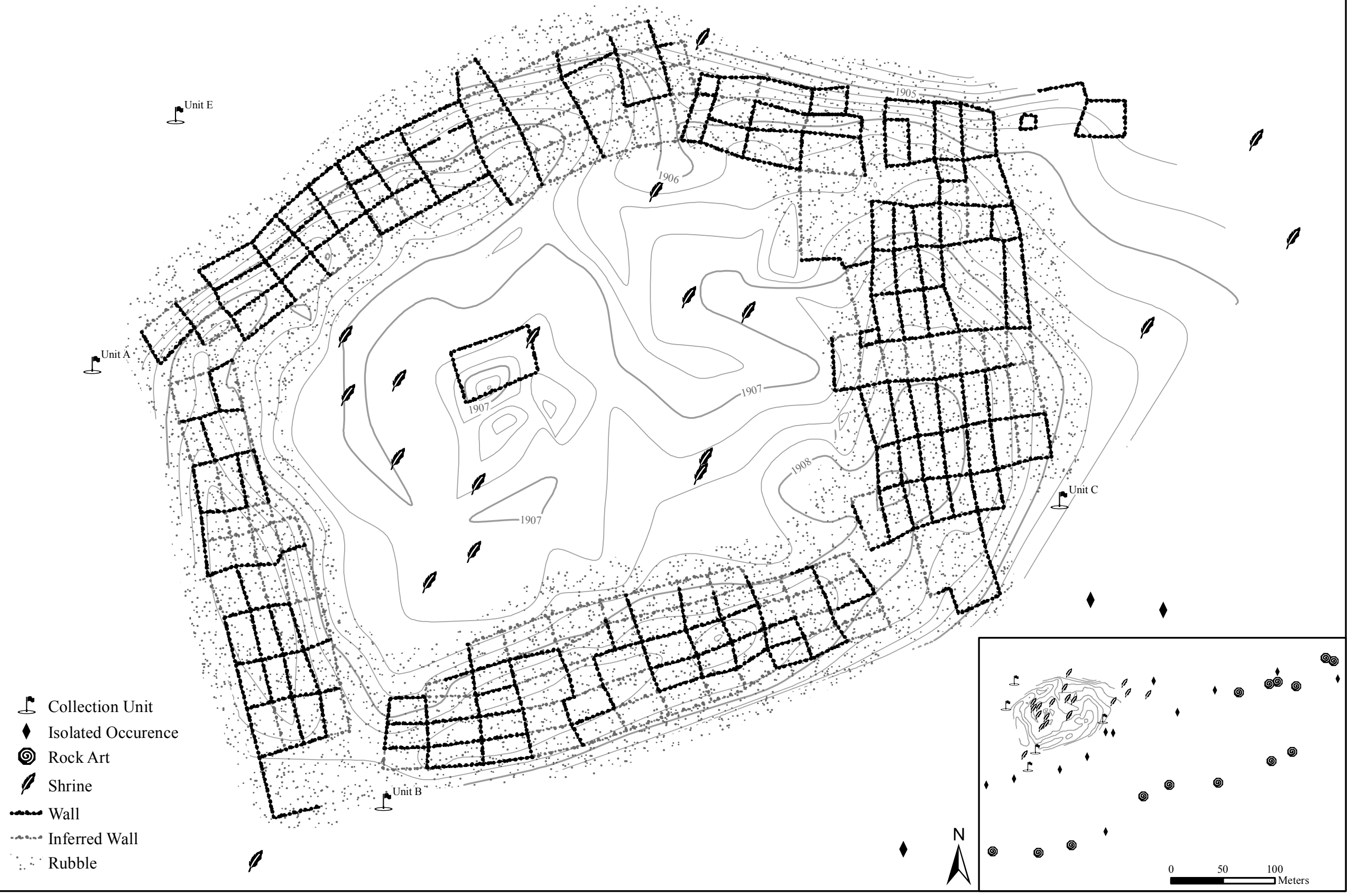




\section{\begin{tabular}{|l|l}
\hline Pink Arrow & NA1001
\end{tabular}}

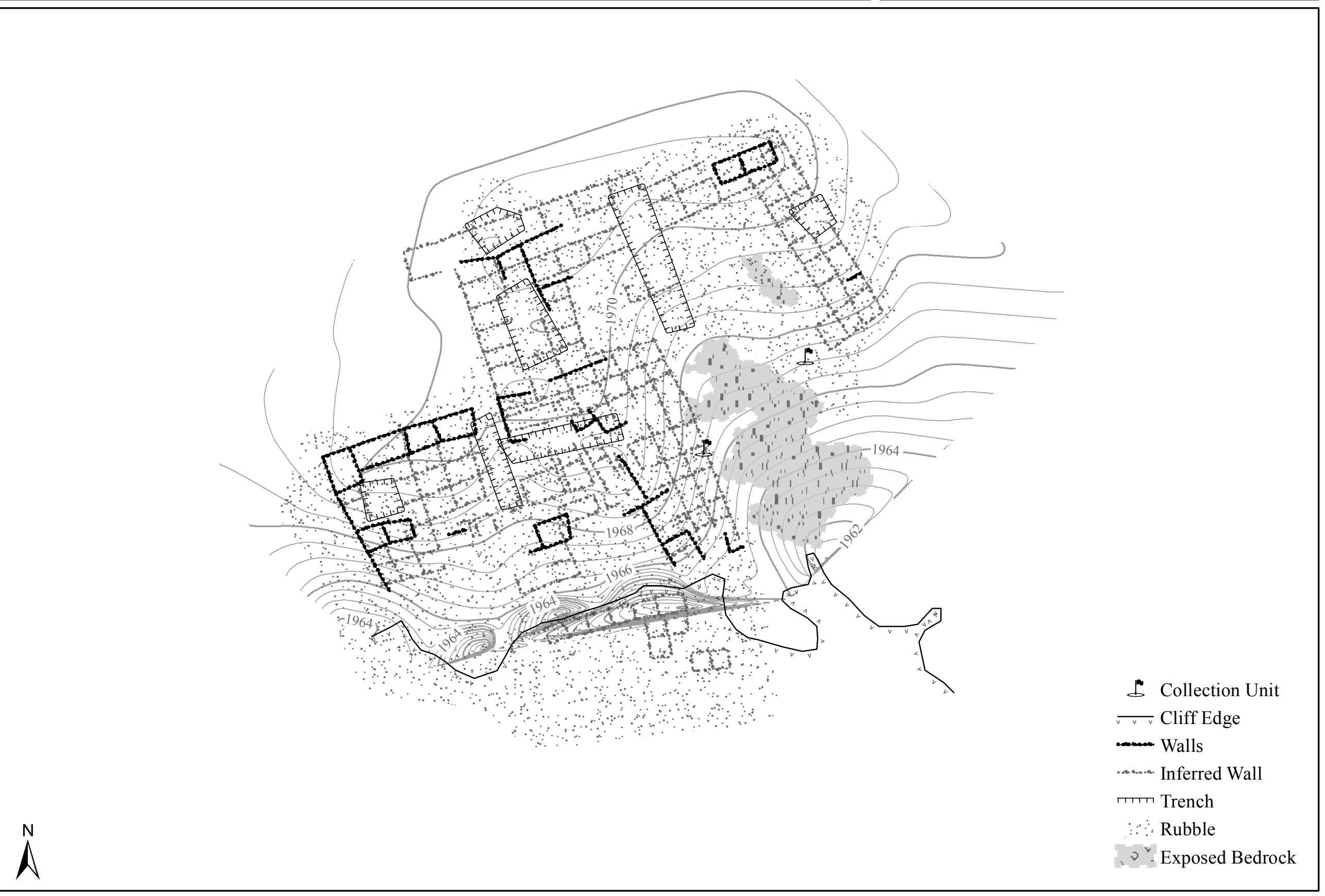




\section{Pivanhonkiopi}

NA1169

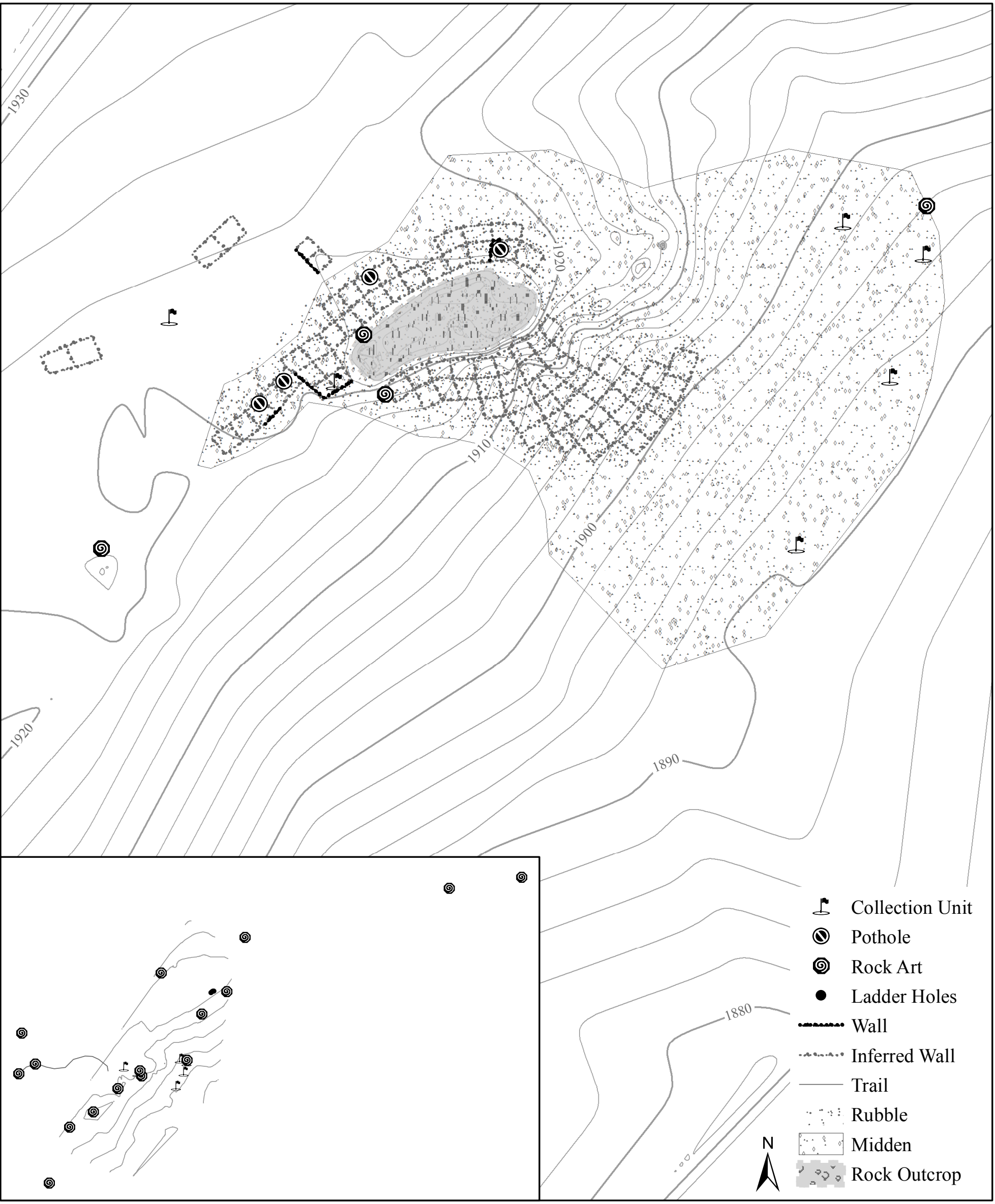




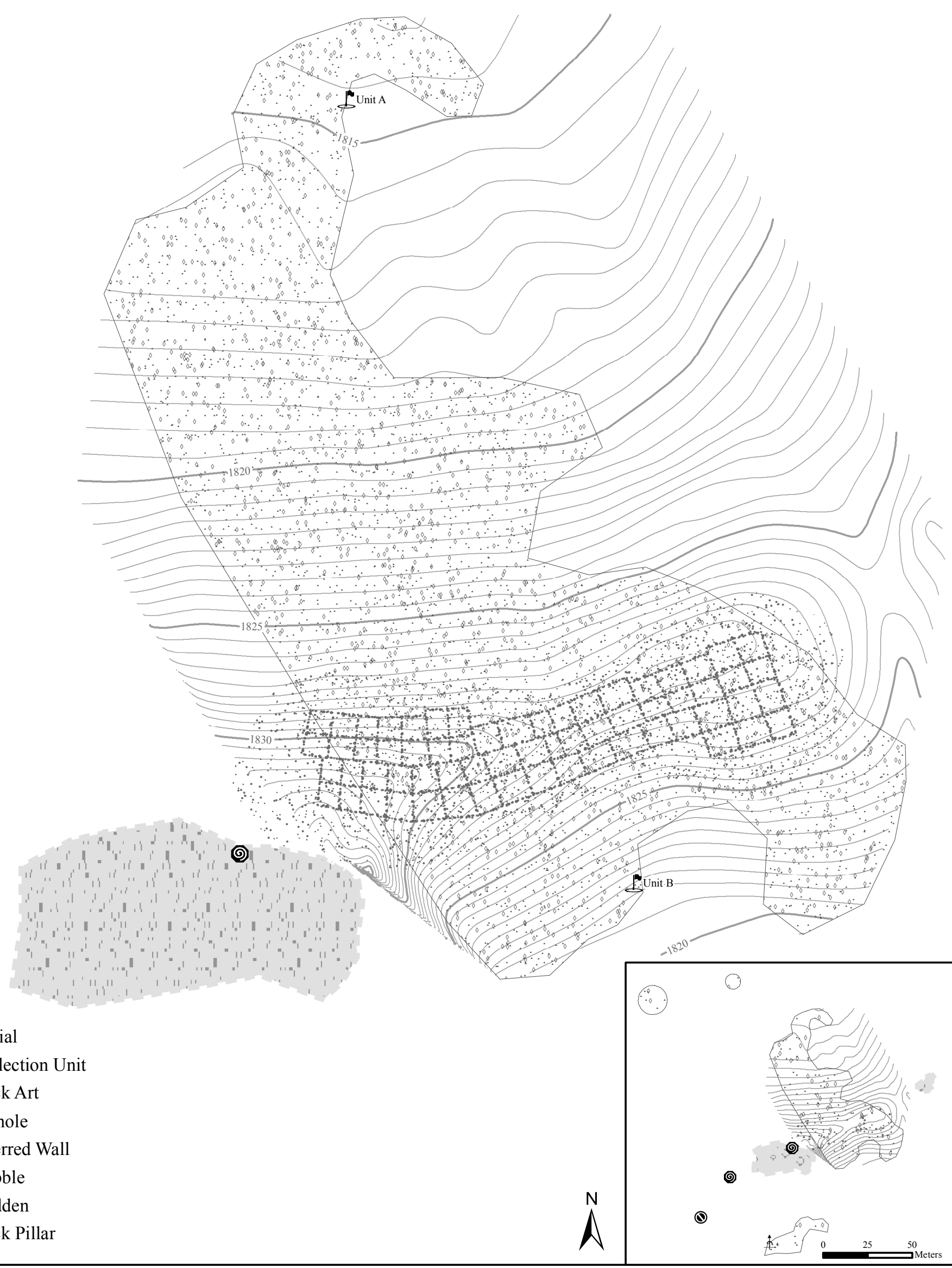

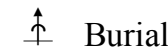

Collection Unit

(Q) Rock Art

(1) Pothole

......... Inferred Wall

$\therefore$... Rubble

$\because \because$ Midden

$p^{2}$, Rock Pillar 


\section{Qaotaqtipu}

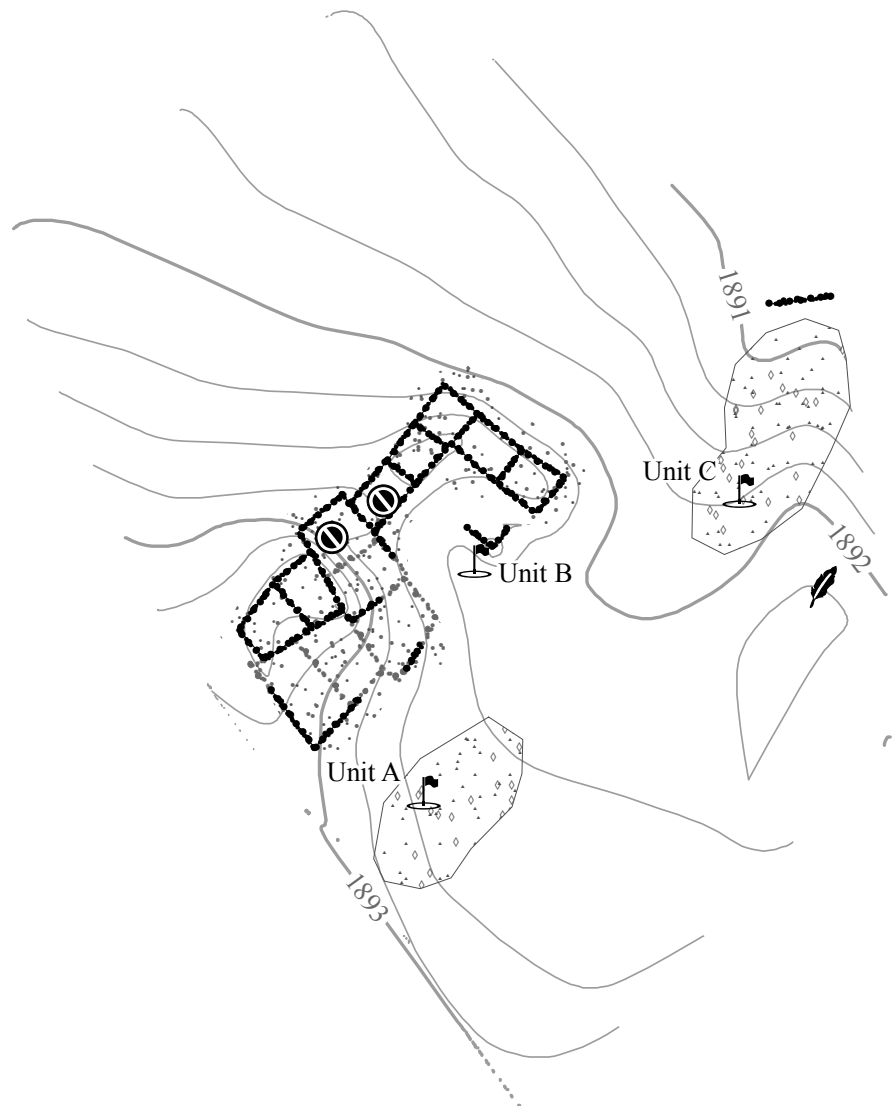

\& Collection Unit

(1) Pothole

$\varangle$ Shrine

- Wall

........ Inferred Wall

$\because$ Rubble

$\therefore$. Midden 


\section{Qootsaptuvela}

NA1699

(Q)

(Q)

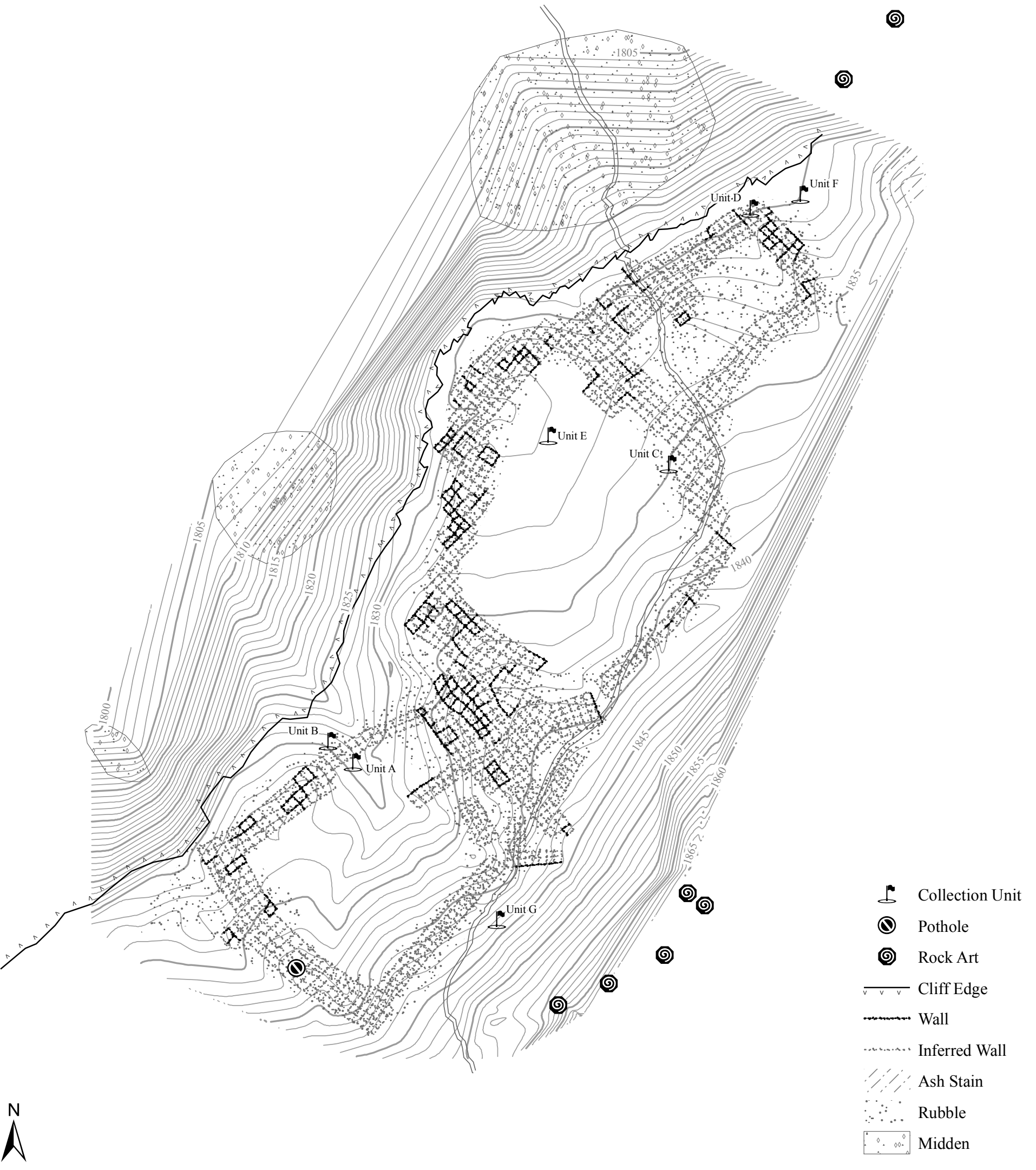




\section{Sanovi}

NA28332
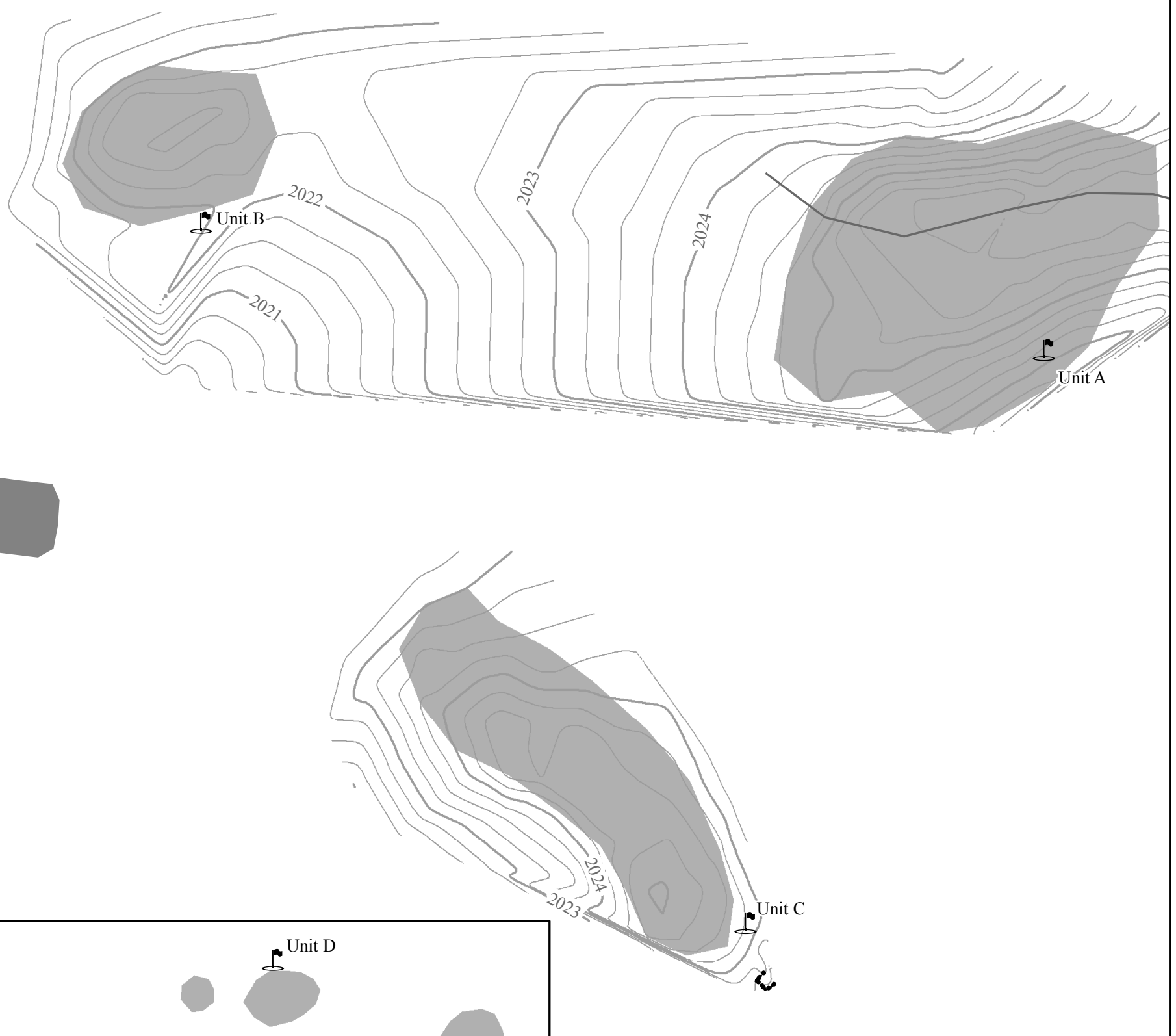

$\omega^{\text {Unit B }}$
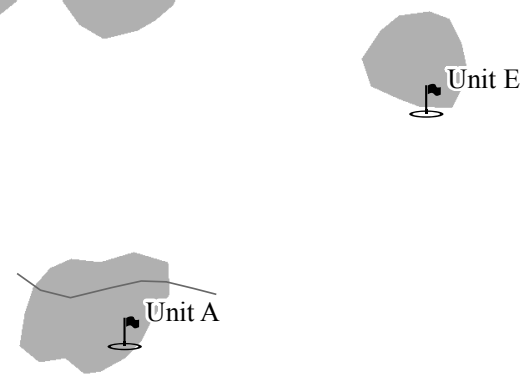

\& Collection Unit

Road

Wall

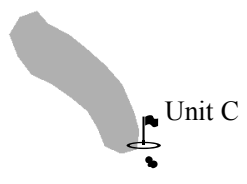

Dance Plaza
Residential Area




\section{West Sanovi}

NA28332

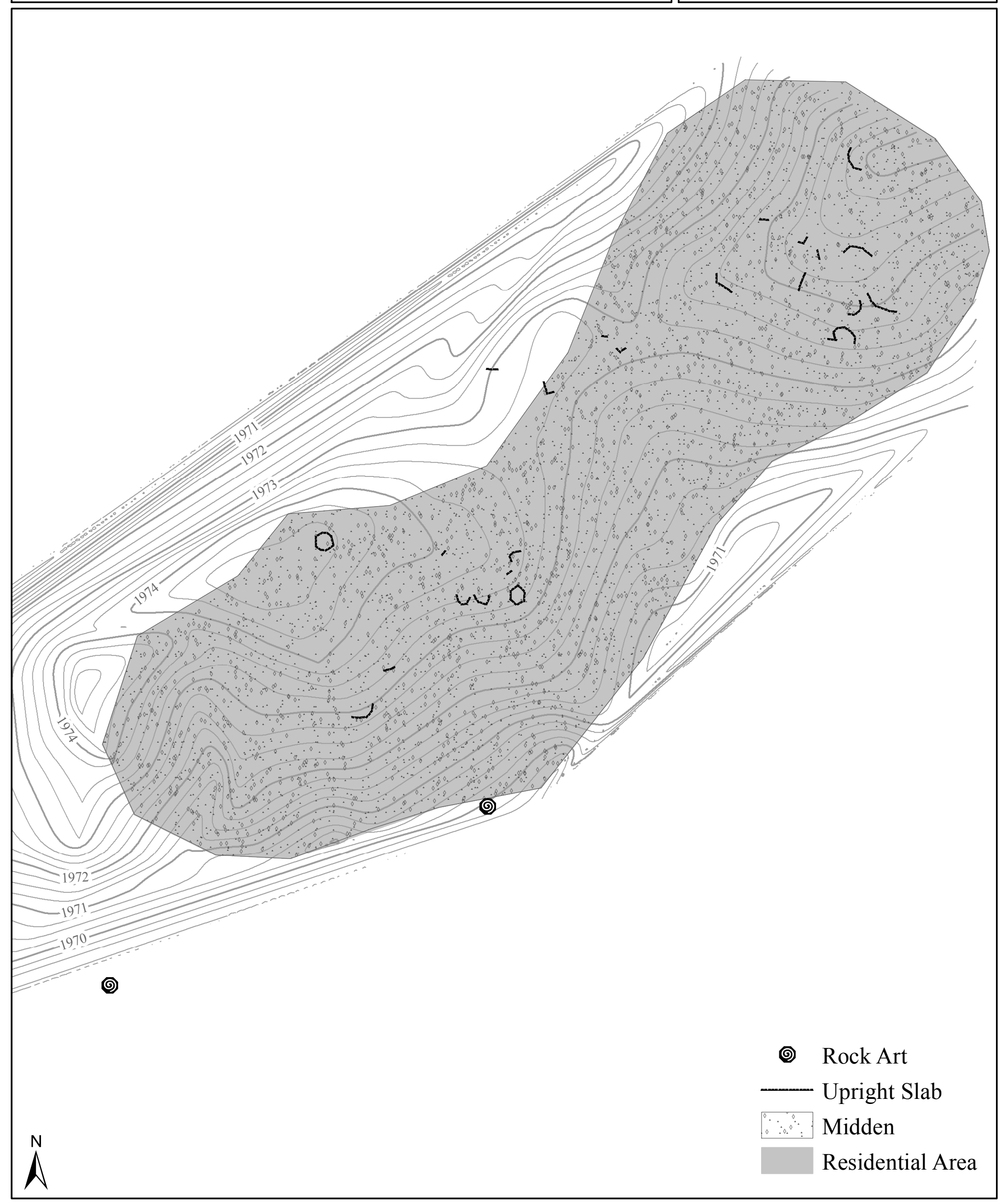




\section{West Sanovi}

NA28332

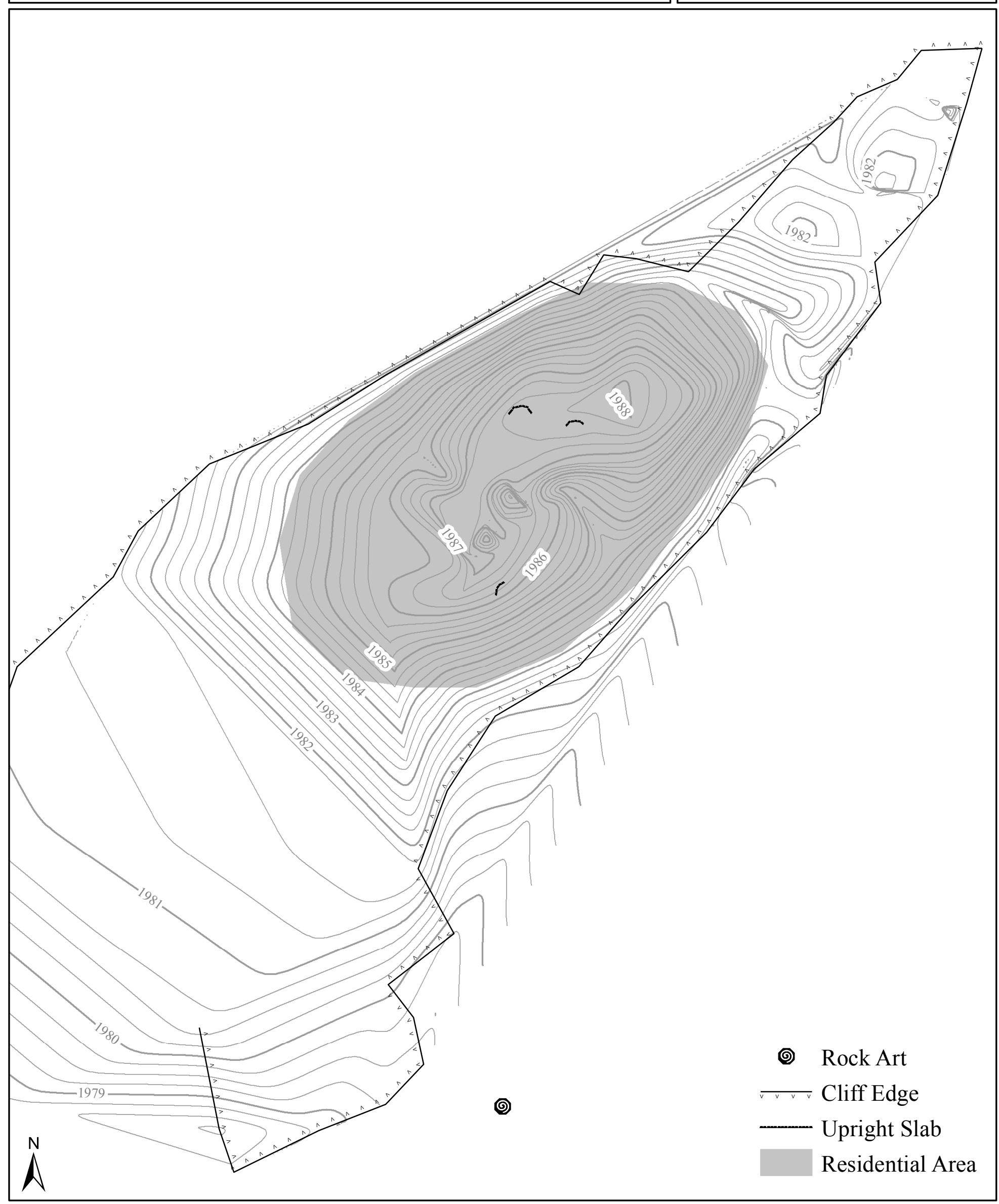




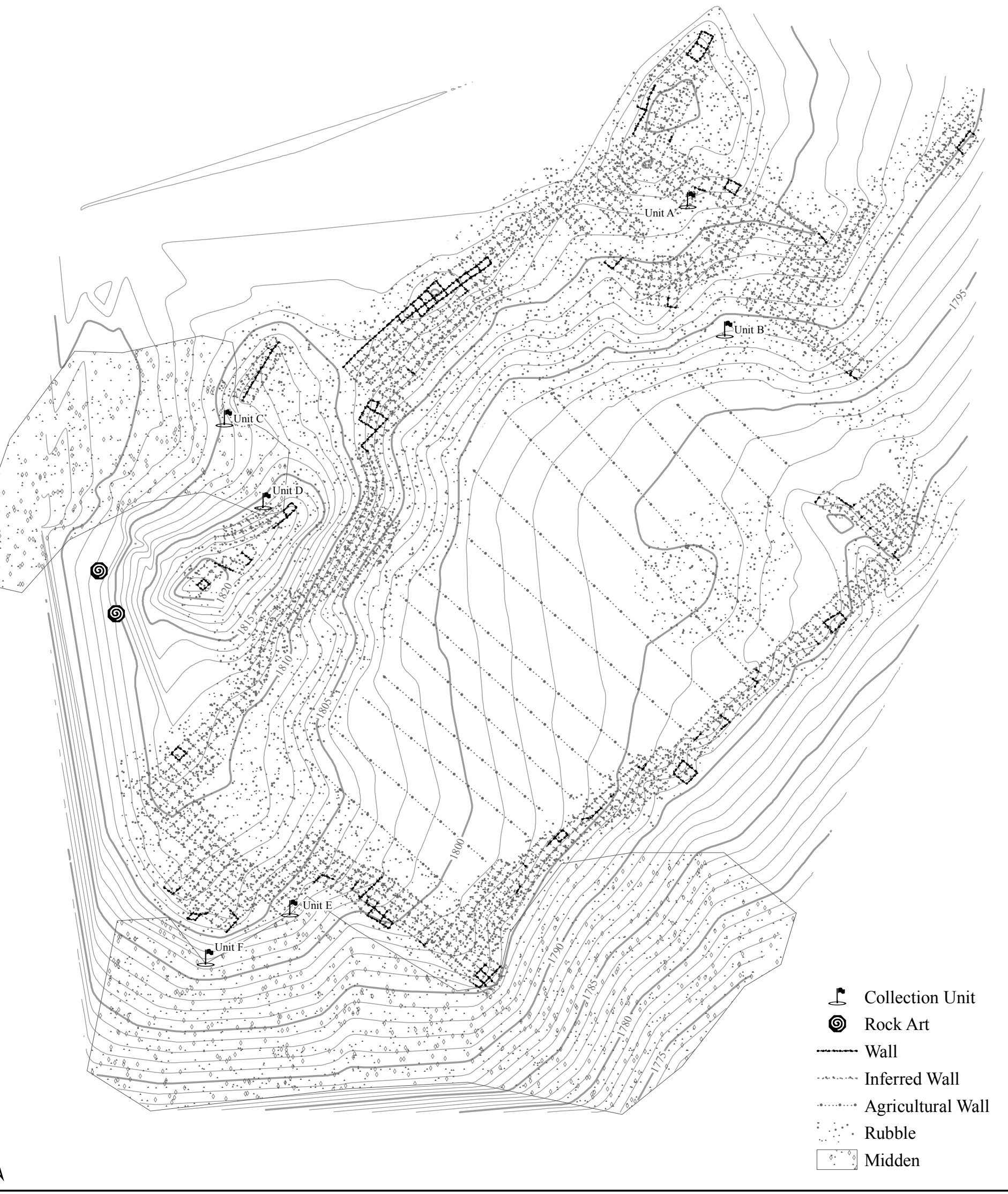




\section{Old Soongopavi}

NA868

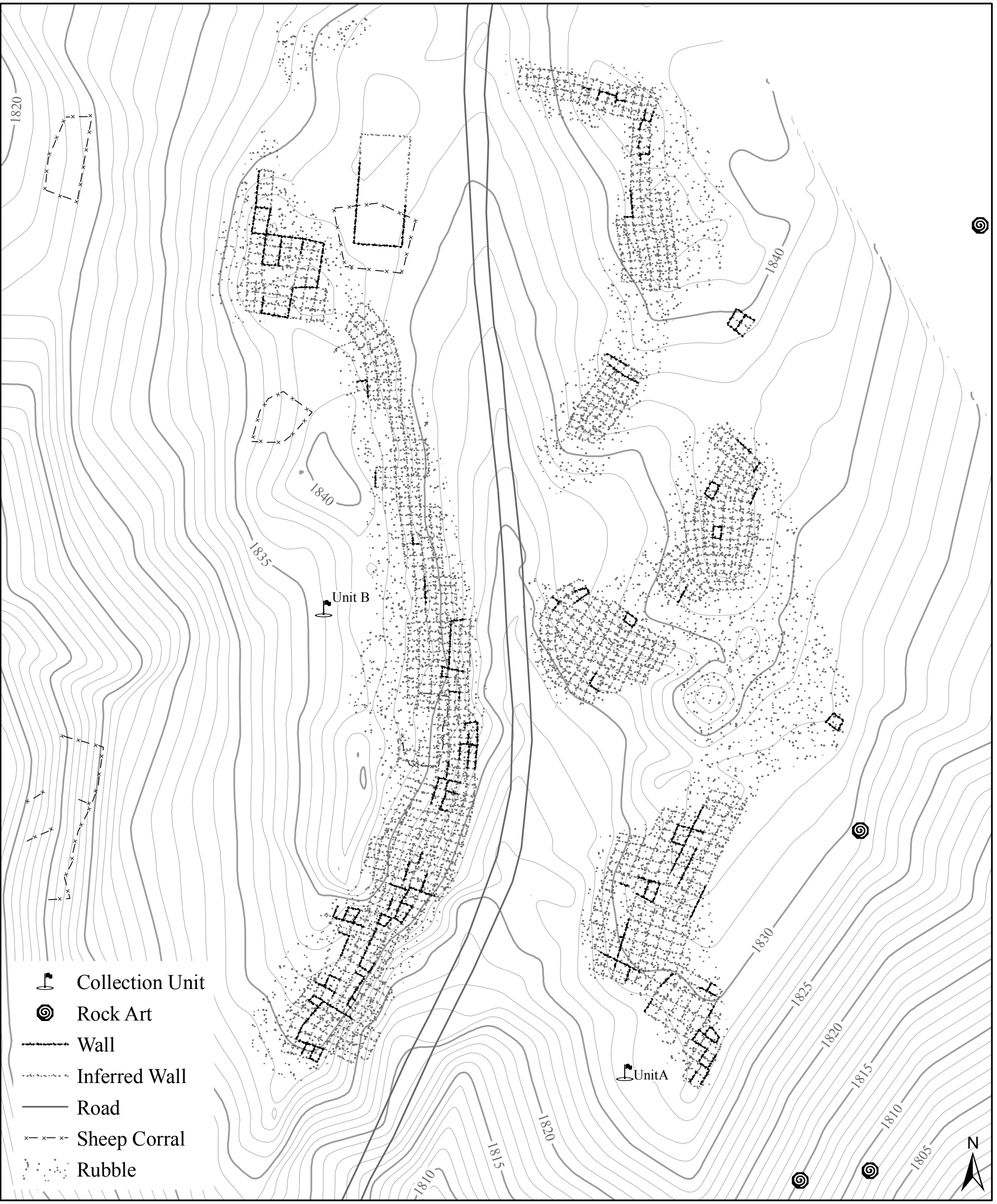




\section{Old Soongopavi \\ NA868}

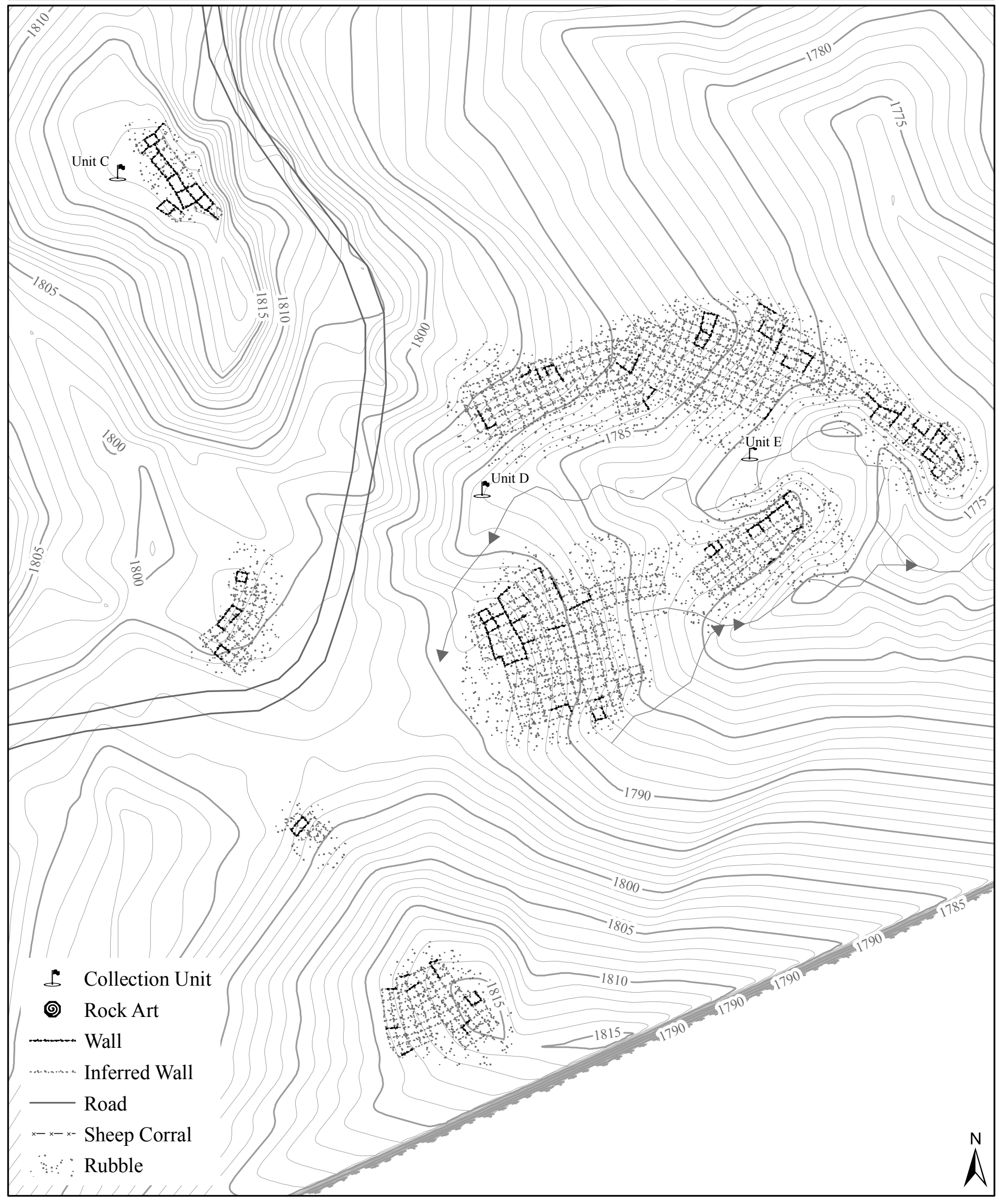




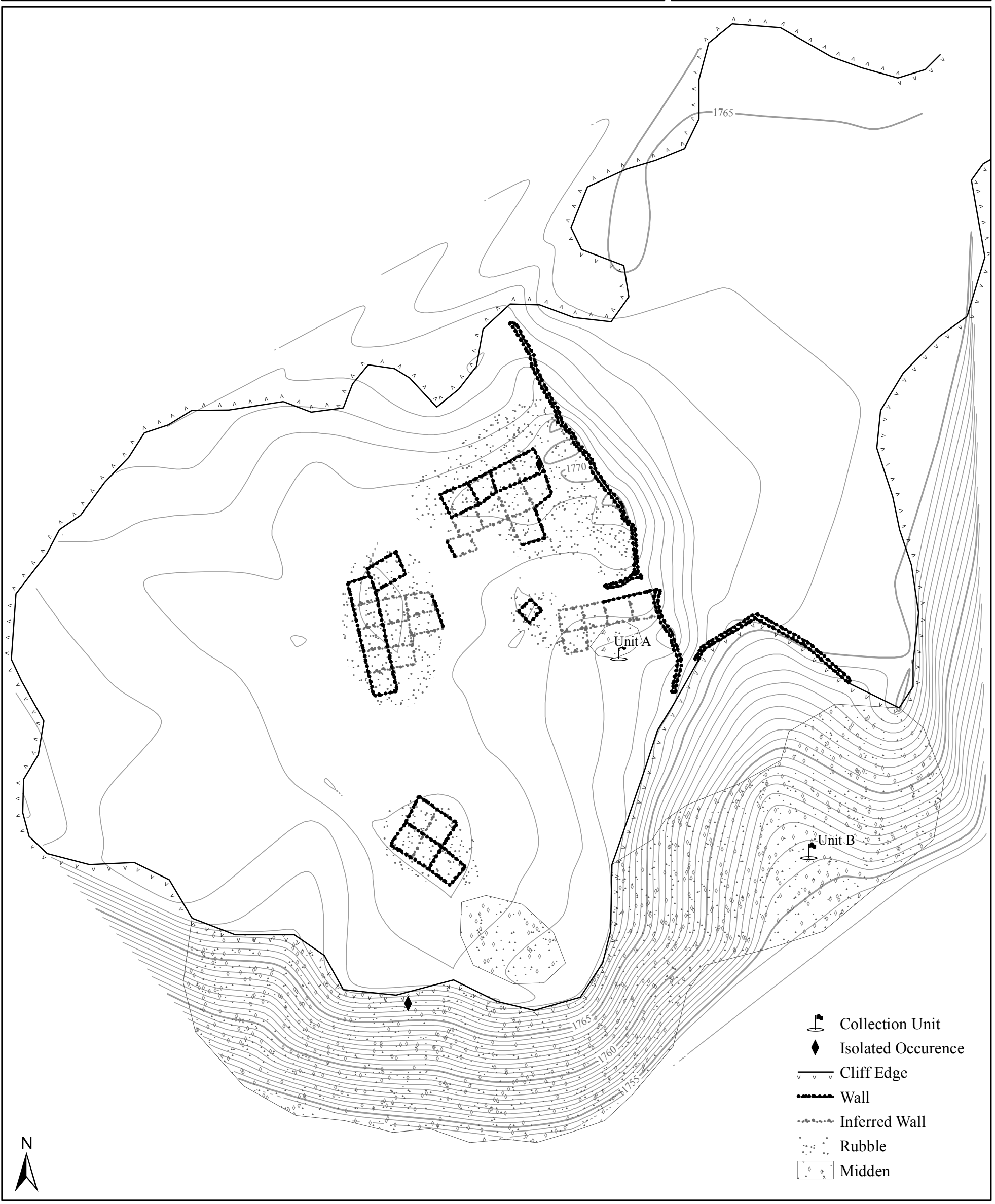




\section{Sun Forehead}

NA9502

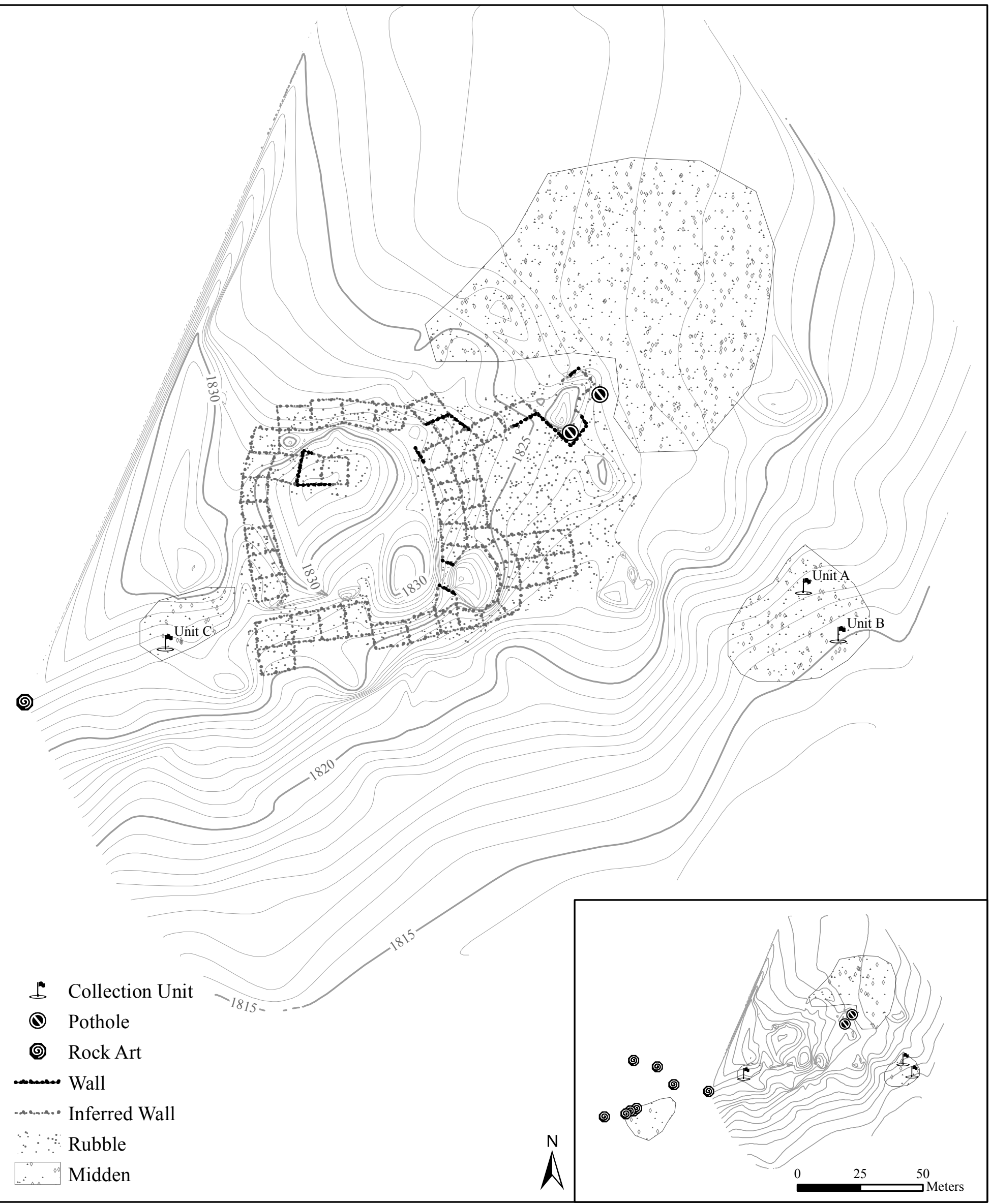




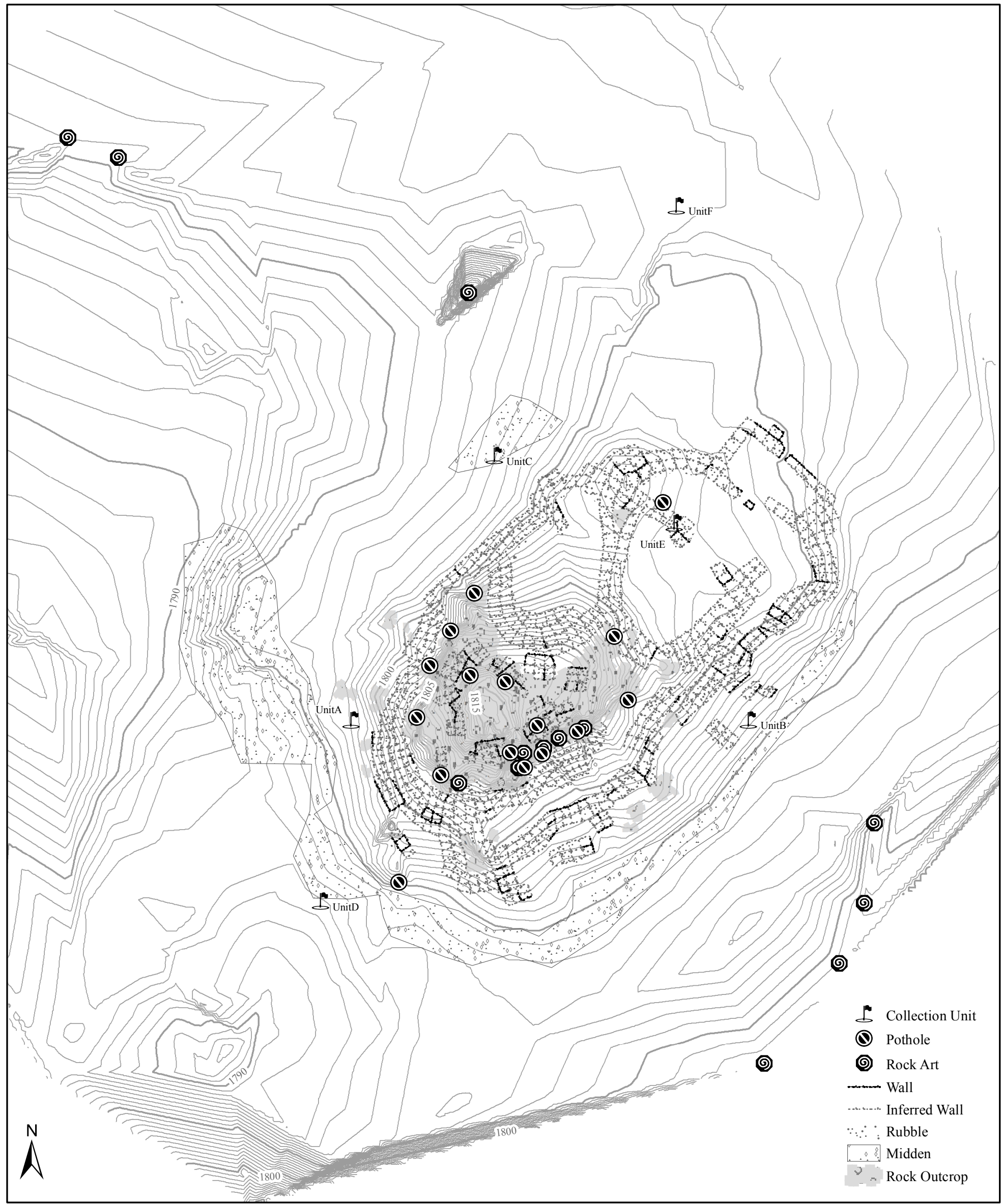




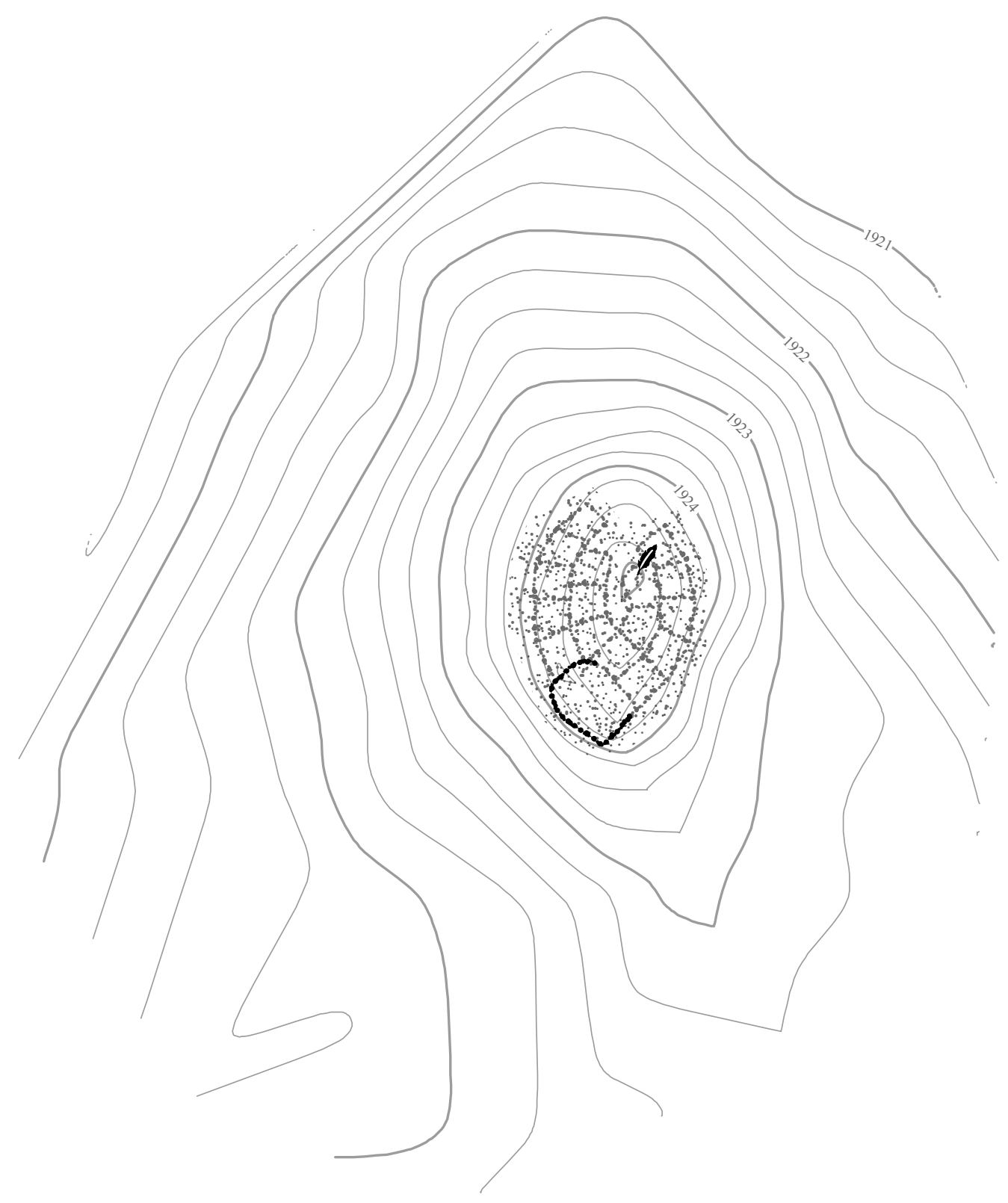

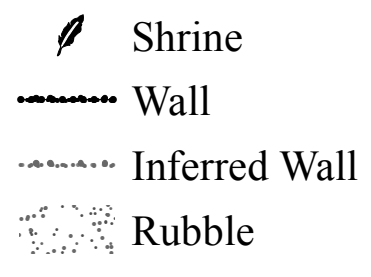




\section{Truskovi}

NA1132

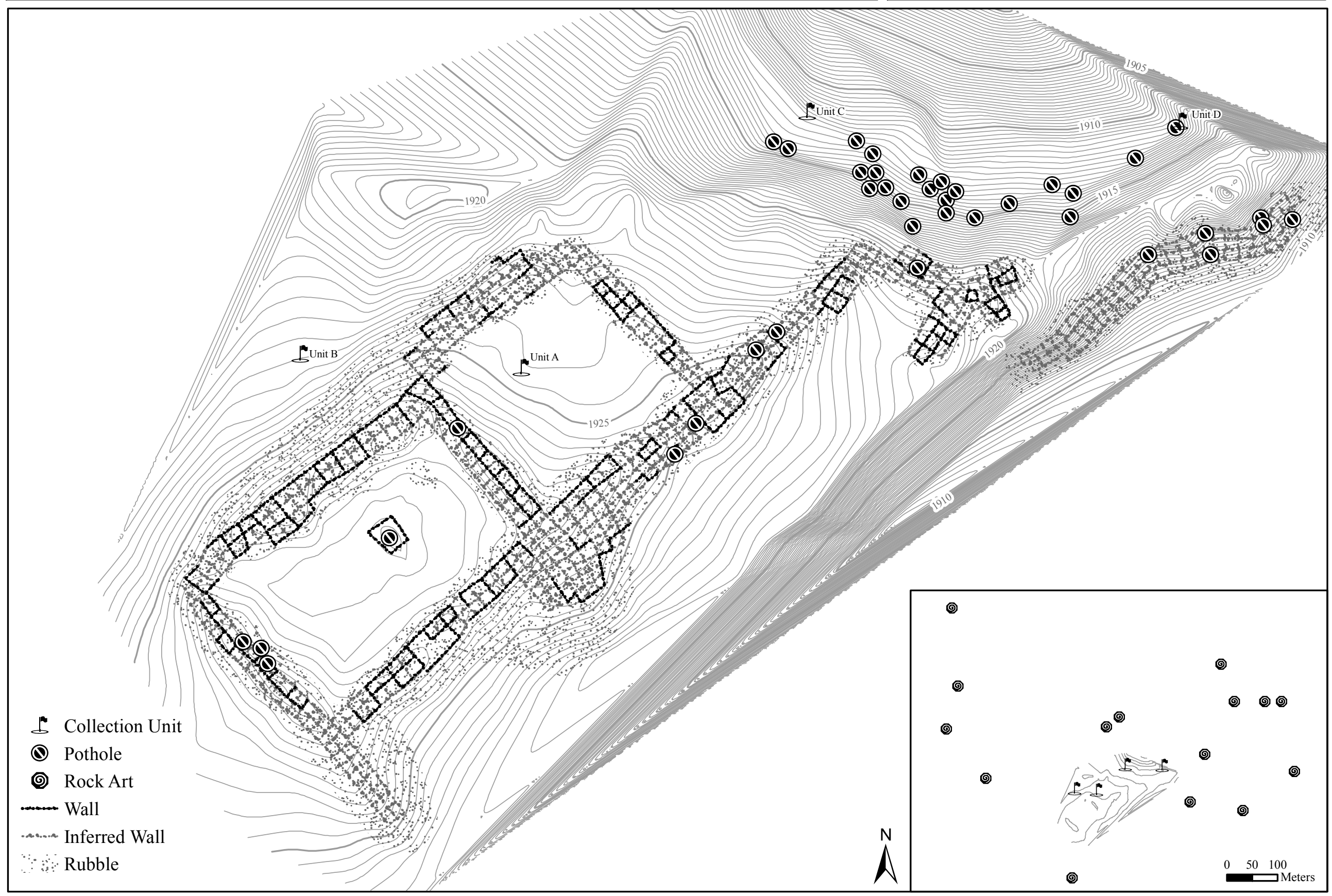




\section{Tsakpahu}

\section{NA1039}

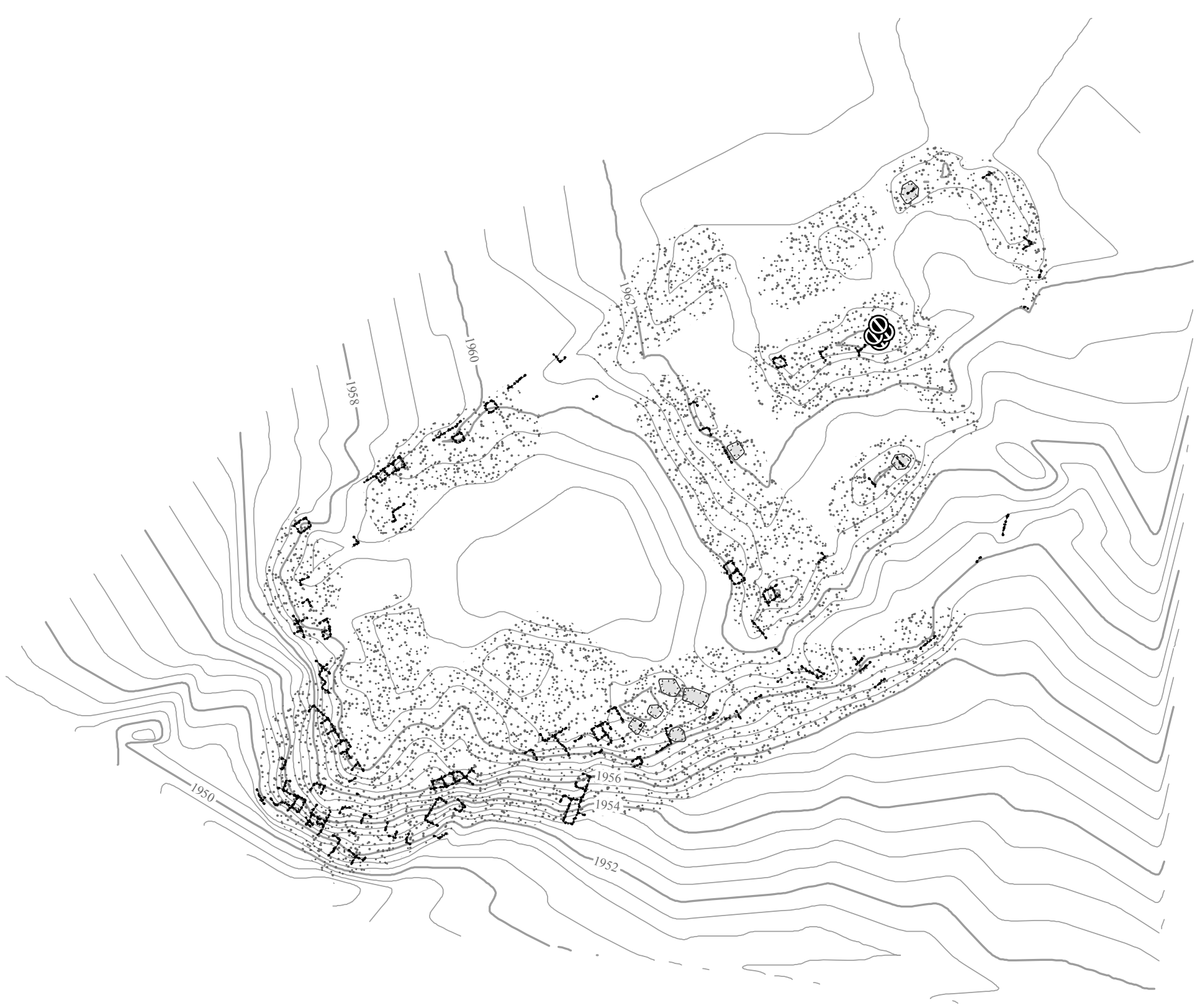




\section{Tsaqkeni \\ NA30662}

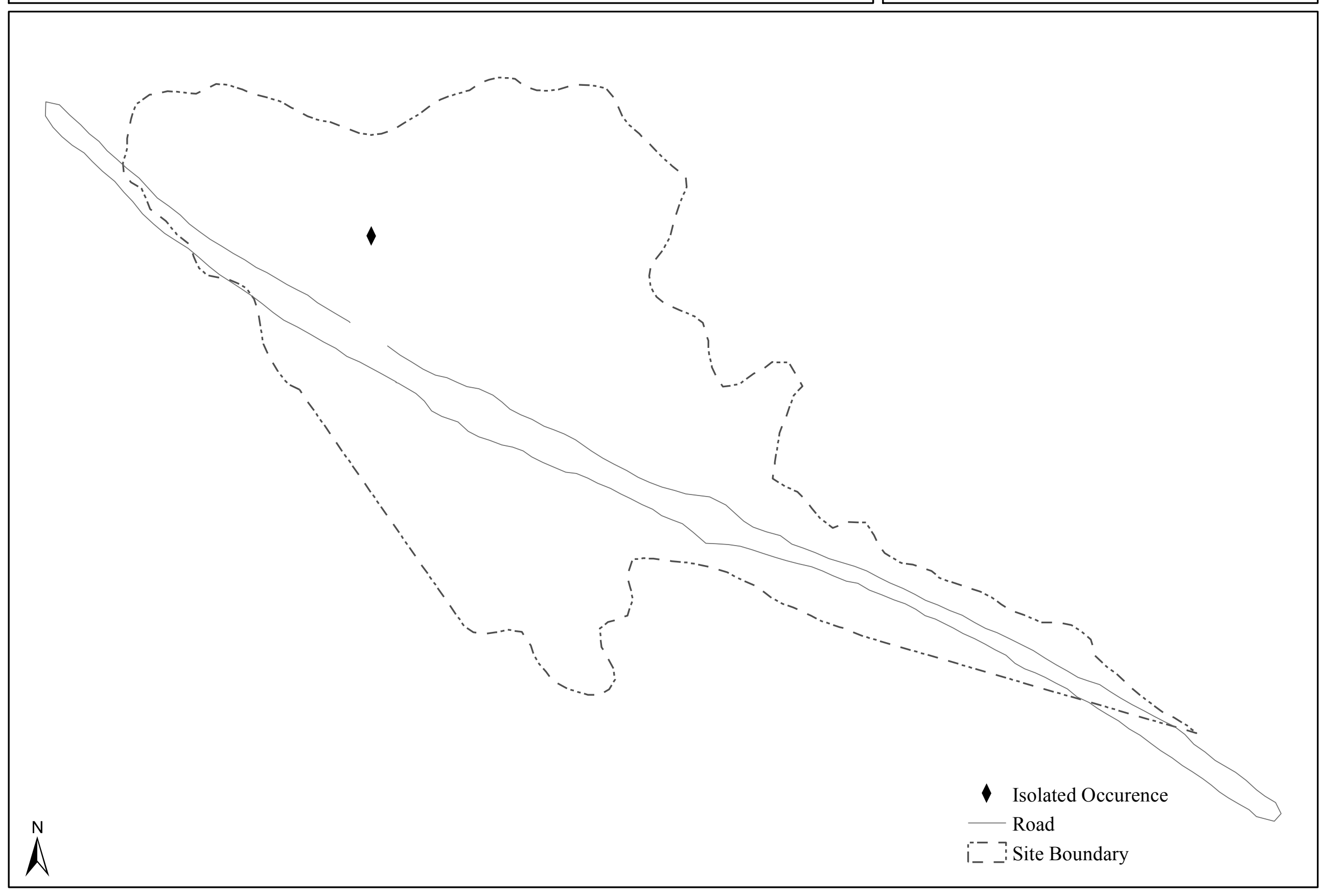



Tukinobi
NA30171

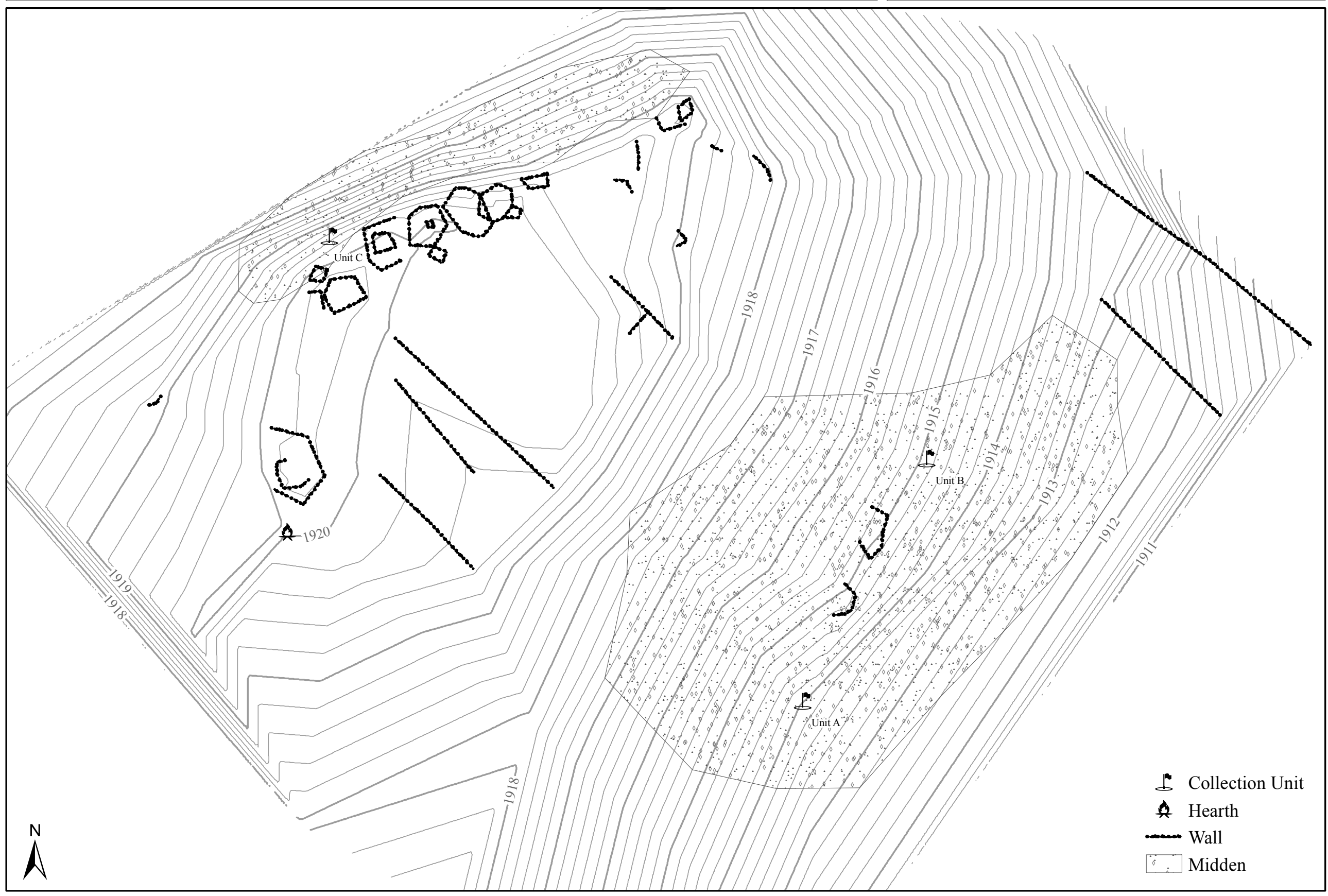




\section{Umtaqa}

NA29406

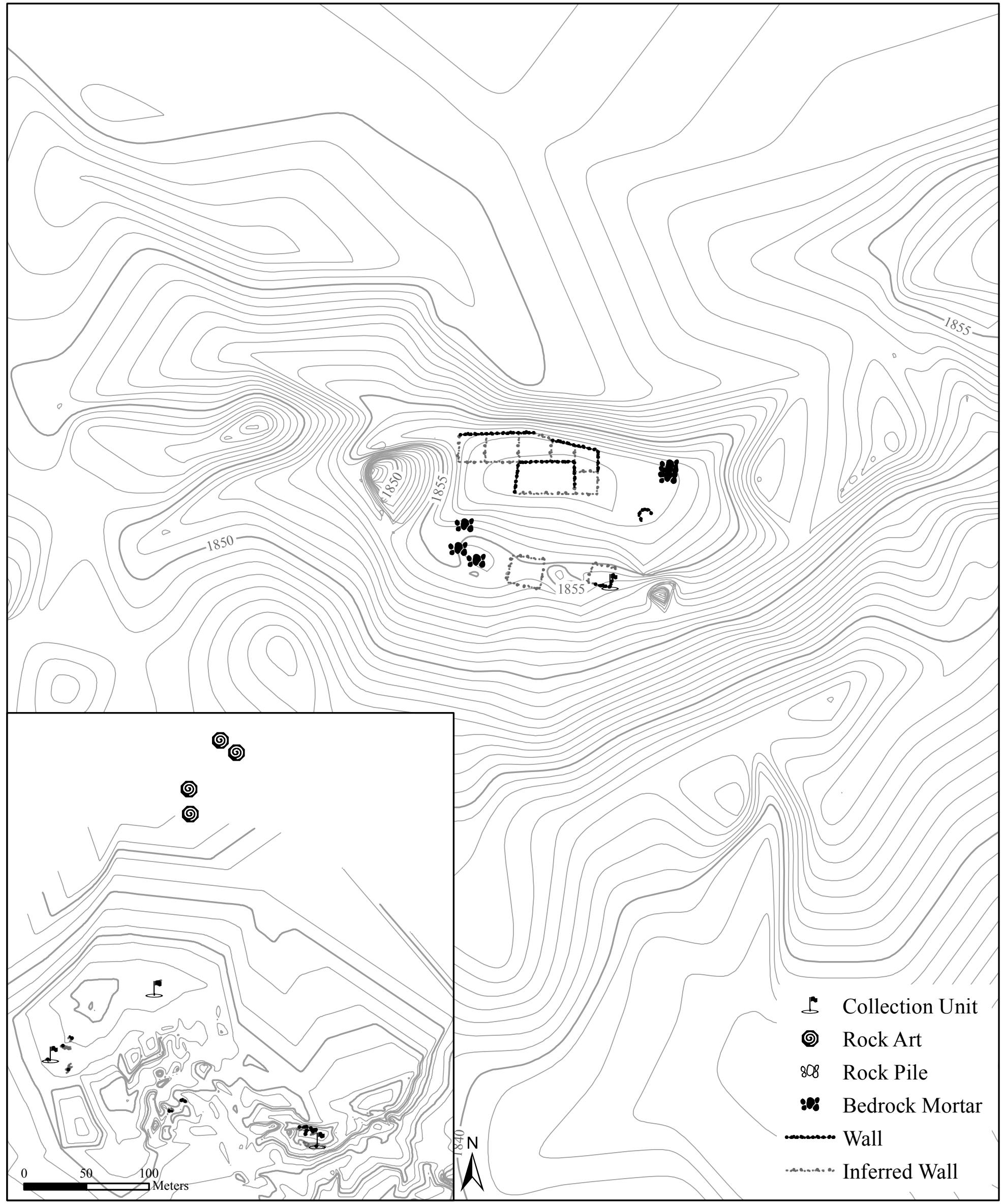

$\begin{array}{lll}0 & 10 & 20 \\ & \end{array}$ 


\section{White Ruin Canyon}

NA30772

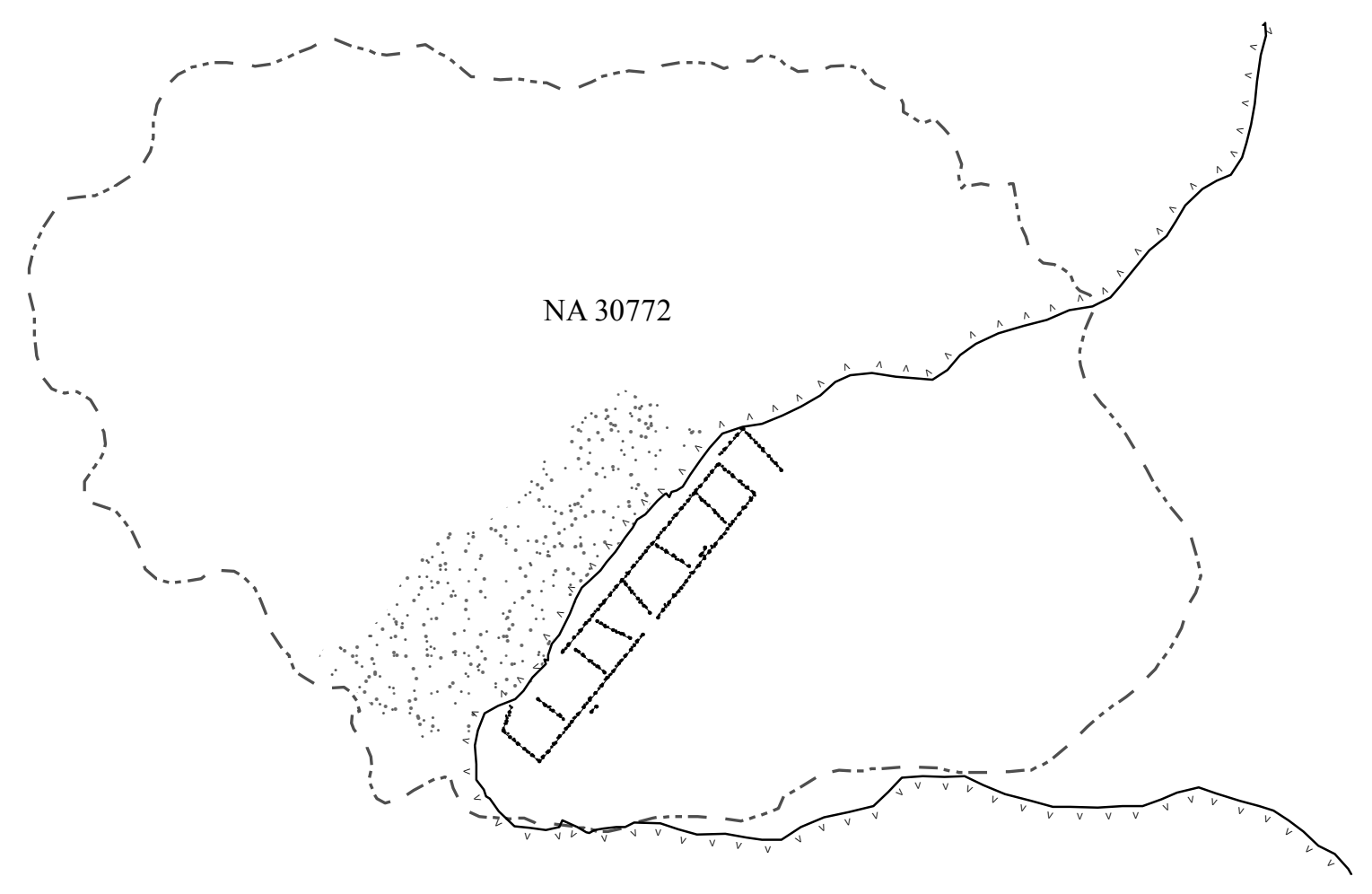

$\lceil$ Cliff Edge

Wall

[- S Site Boundary 


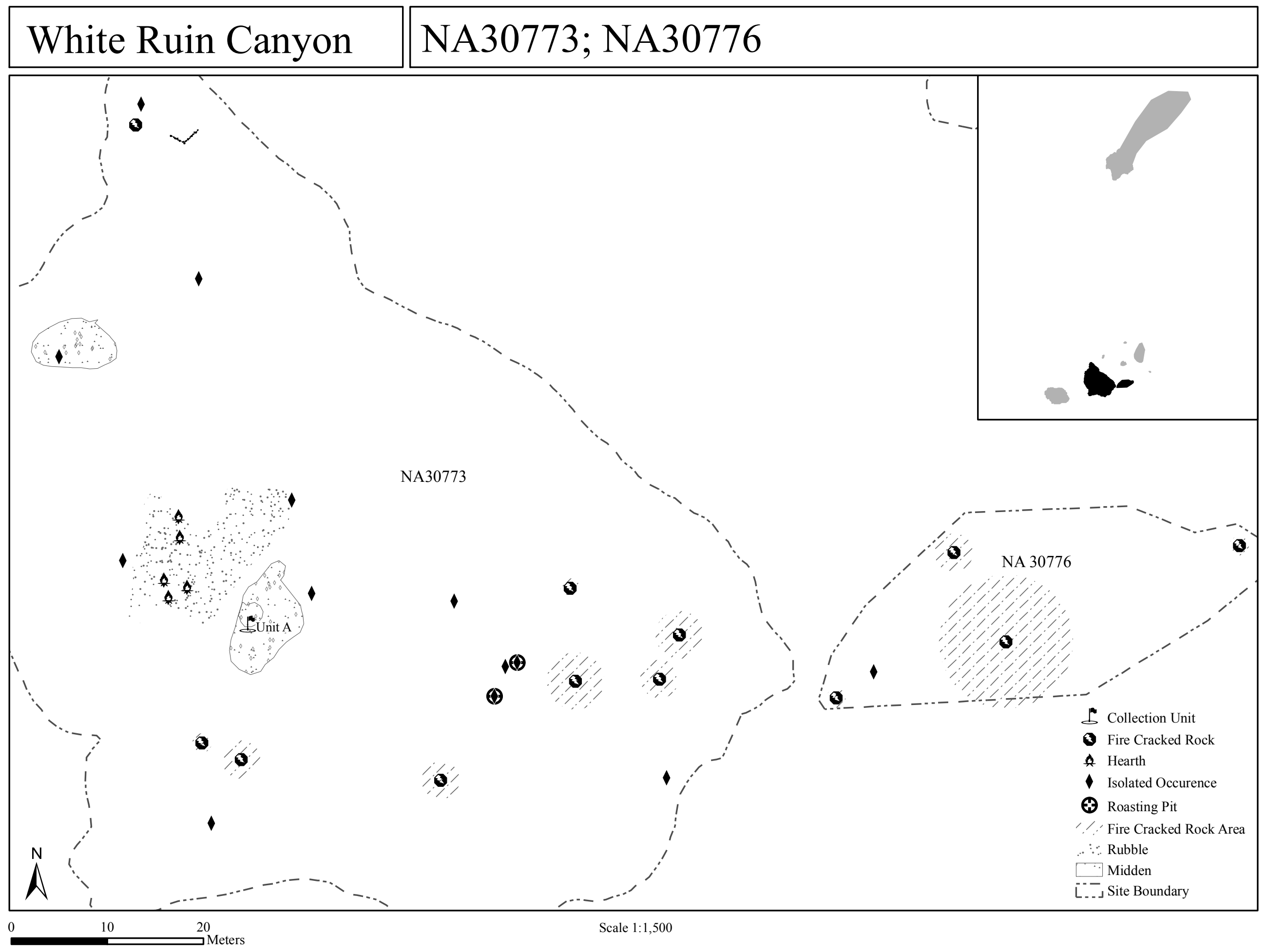




\section{White Ruin Canyon \\ NA30775; NA30808 - NA30810}

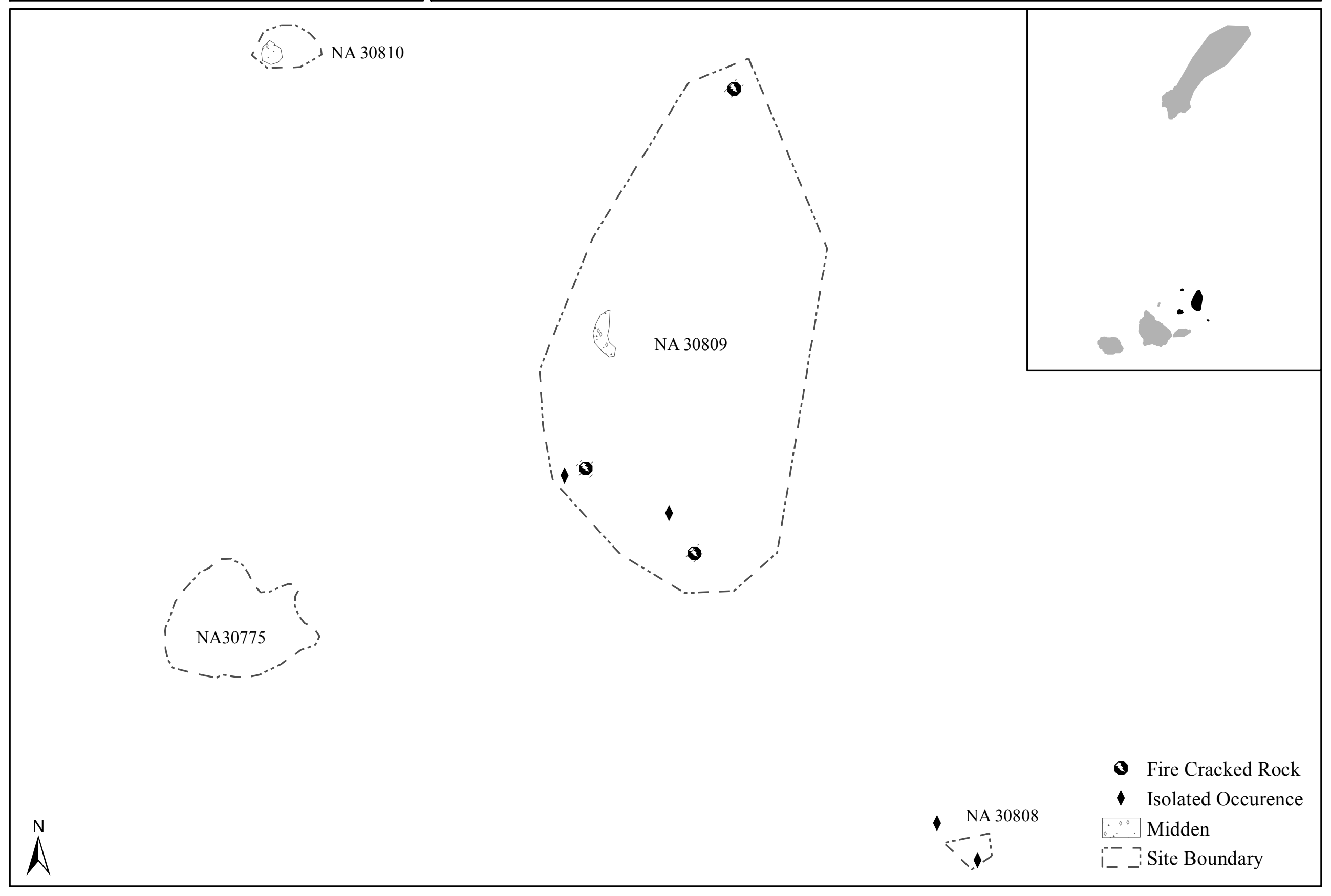




\section{White Ruin Canyon \\ NA30811}

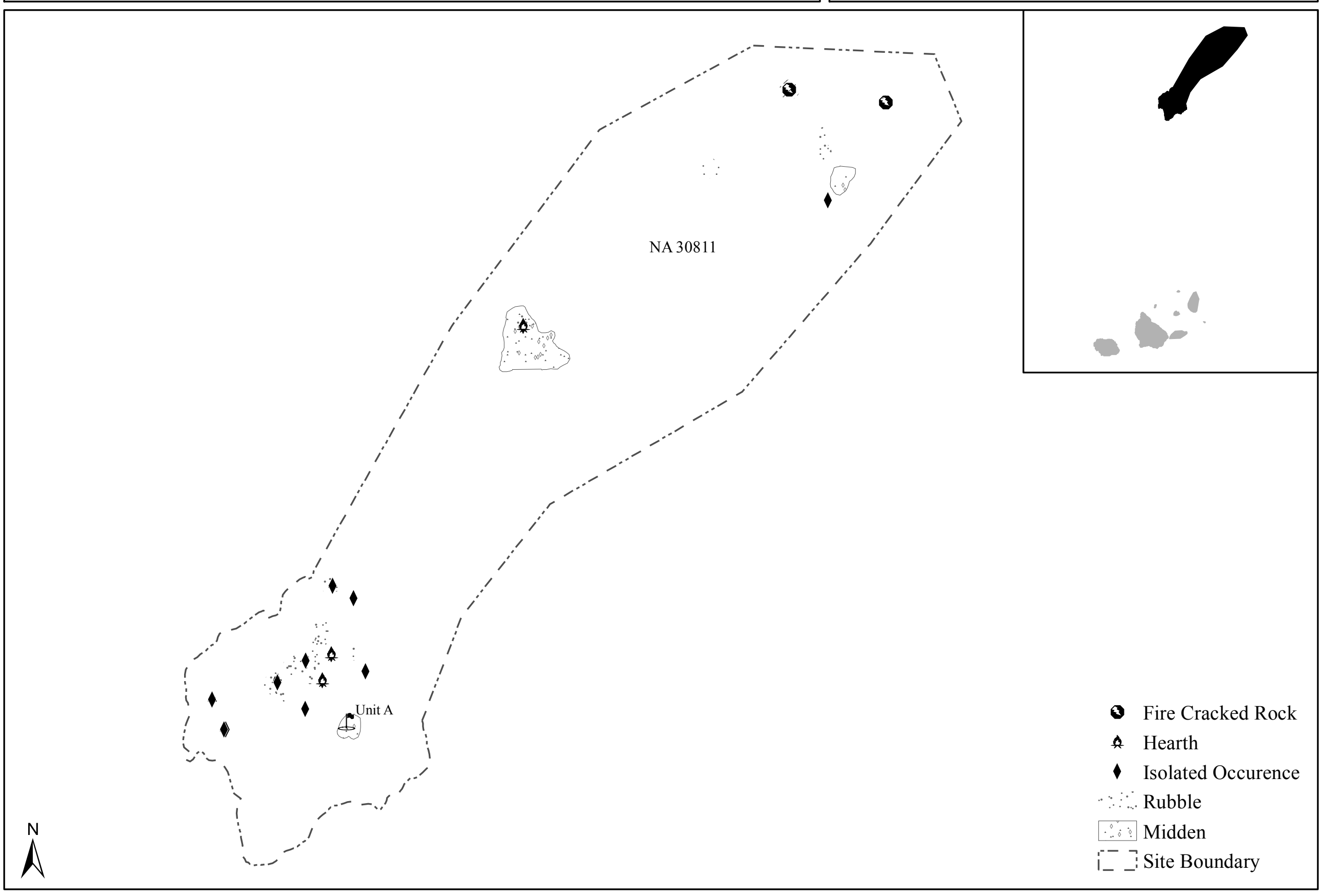




\section{White Spring \\ NA28334}

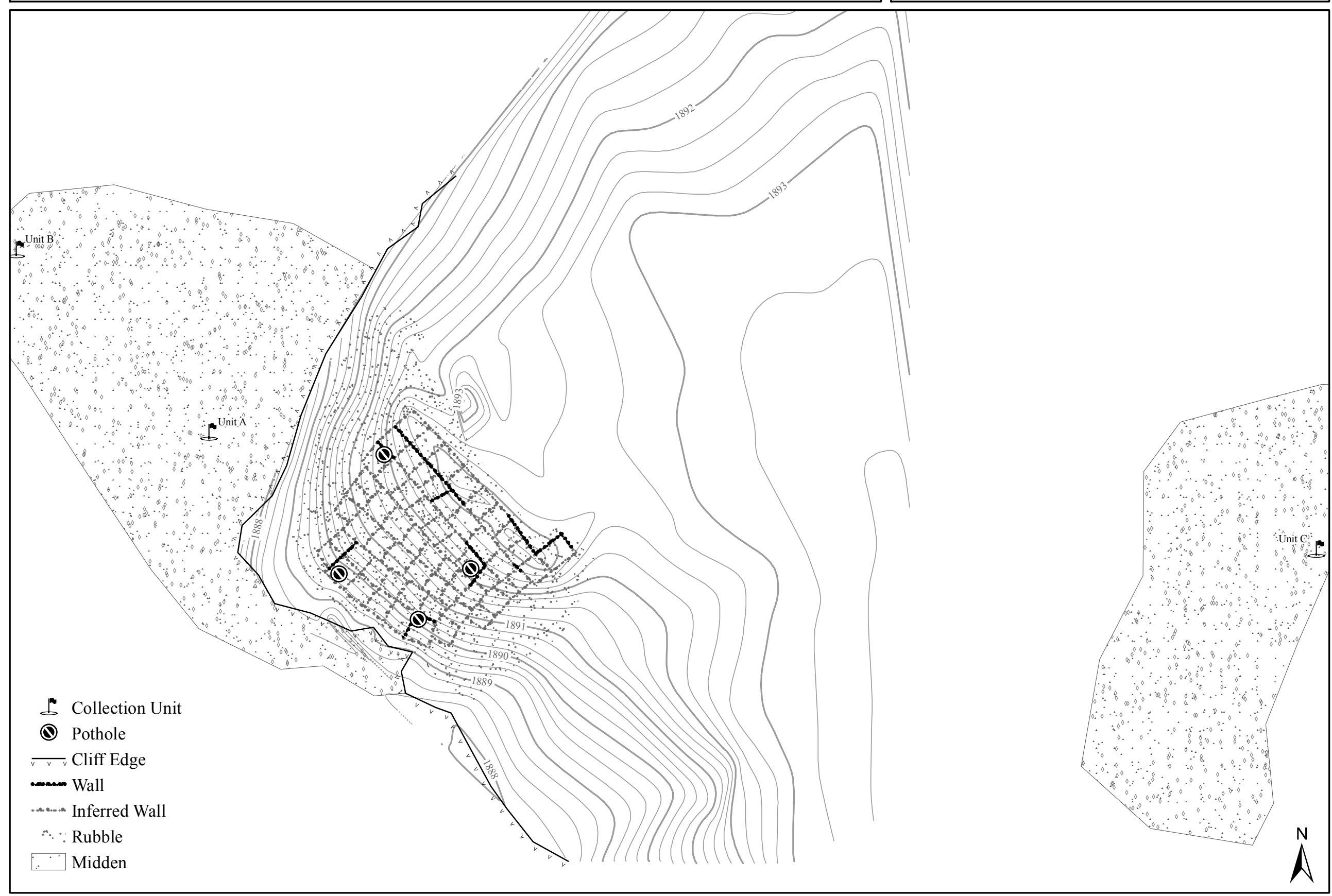




\section{Hopi Reservation Archaeological Sites}
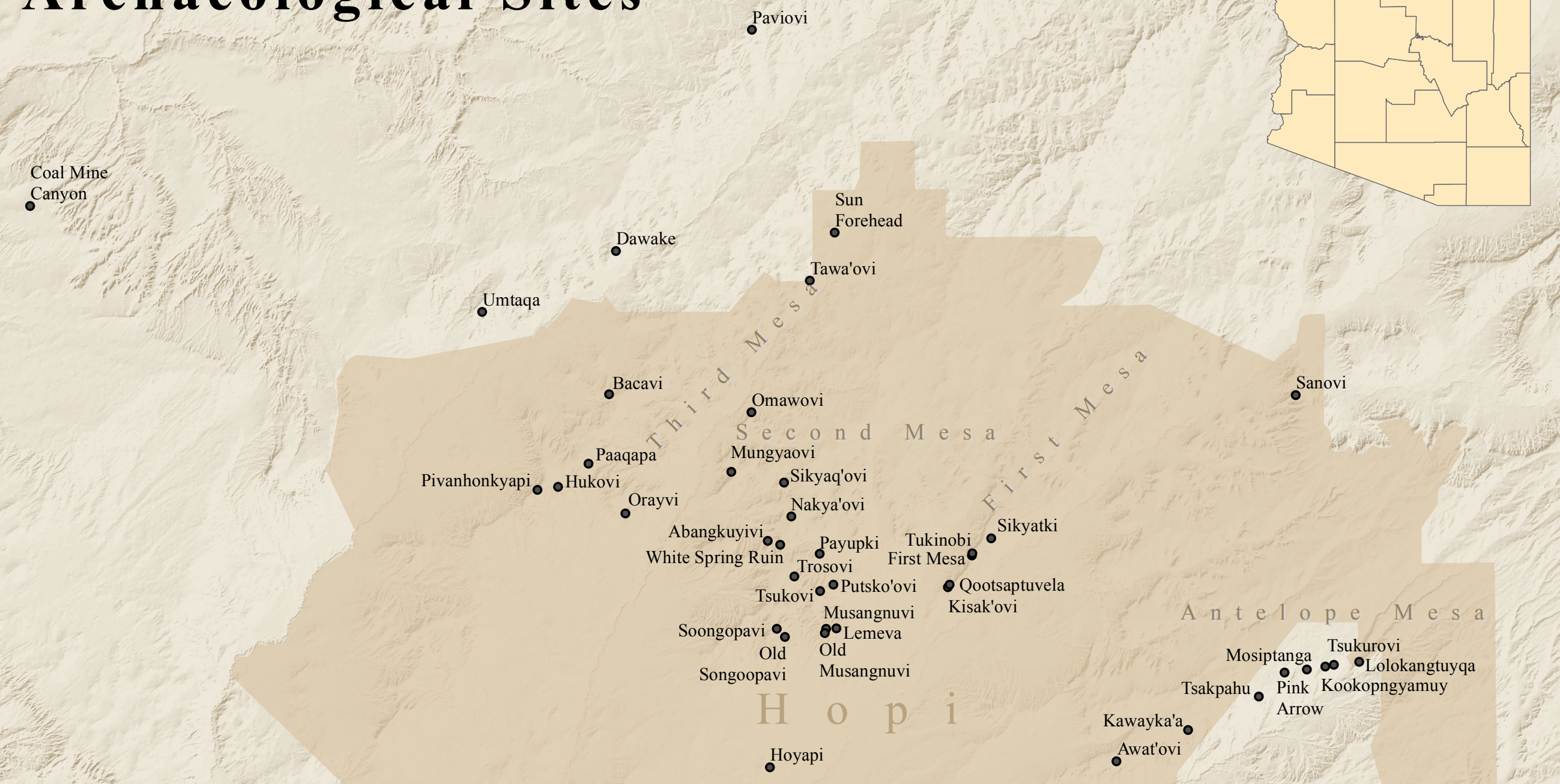

- Huvatuqwa

$0^{\text {Tsakeni }}$

Star 


\section{Abangkuyivi}

NA2299

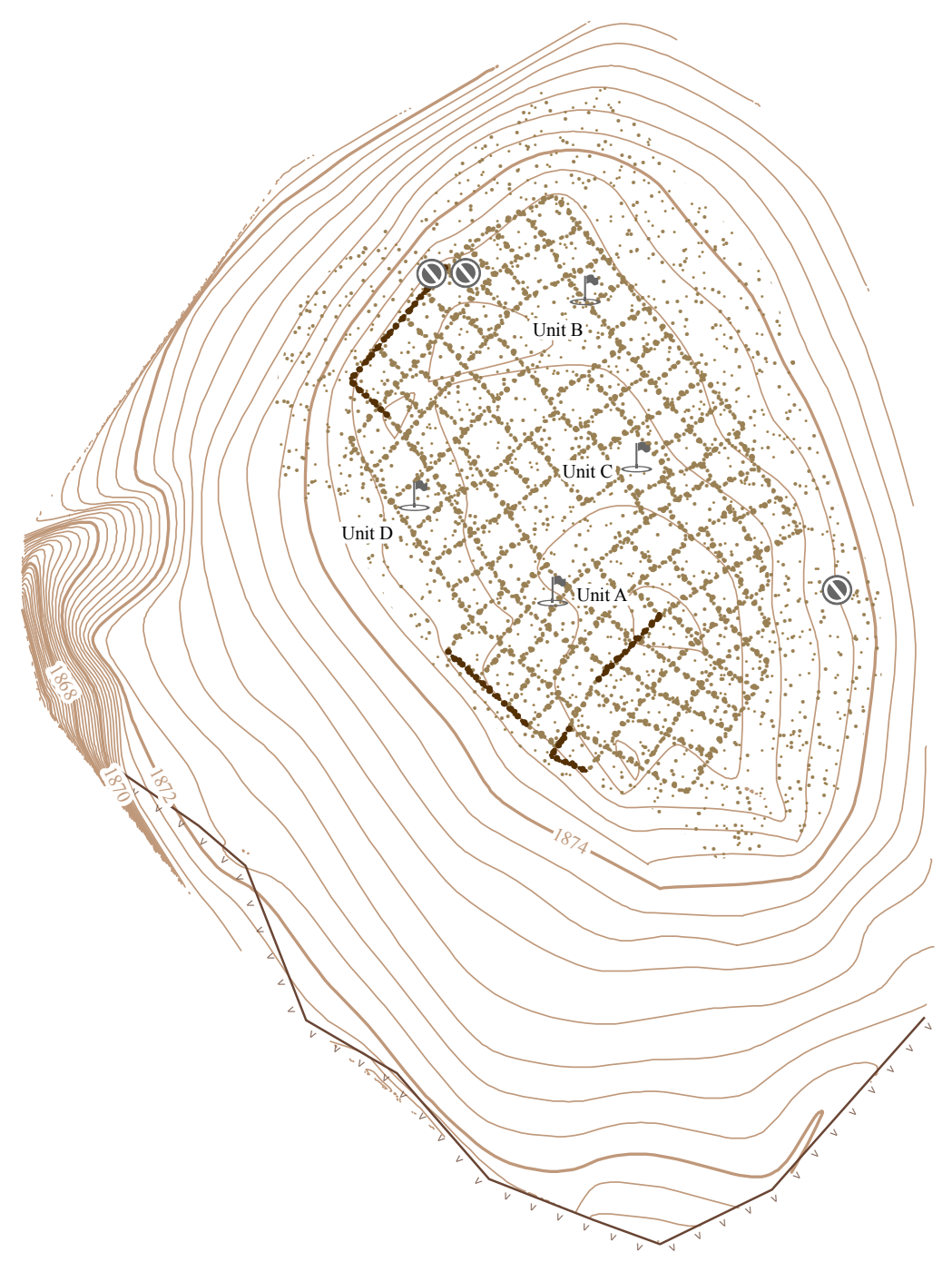

Collection Unit

(a) Pothole

Cliff Edge

- Wall

Inferred Wall

Rubble 


\begin{tabular}{|l|l|}
\hline Awat'ovi & NA820 \\
\hline \hline
\end{tabular}

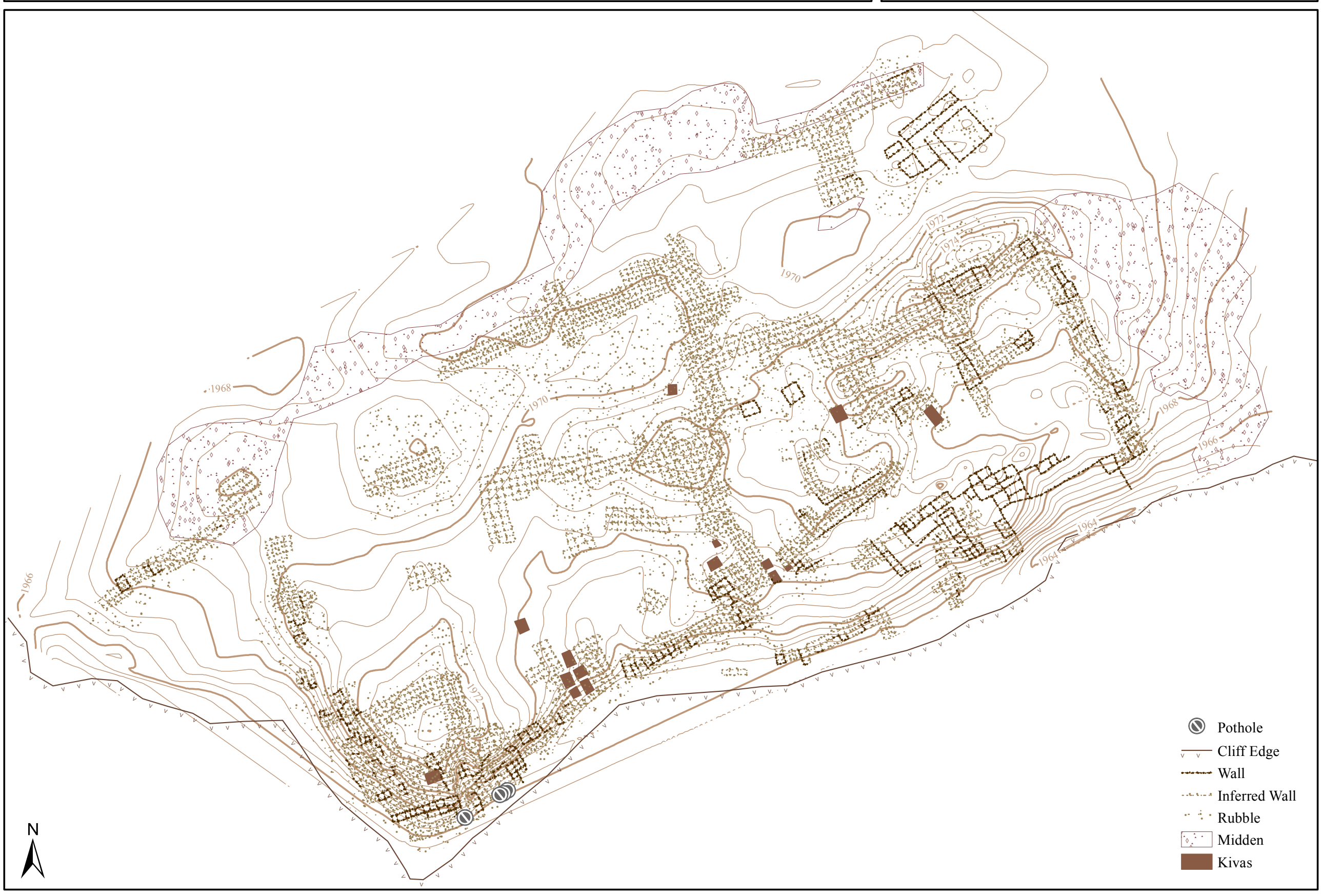




\section{Bacavi Survey}

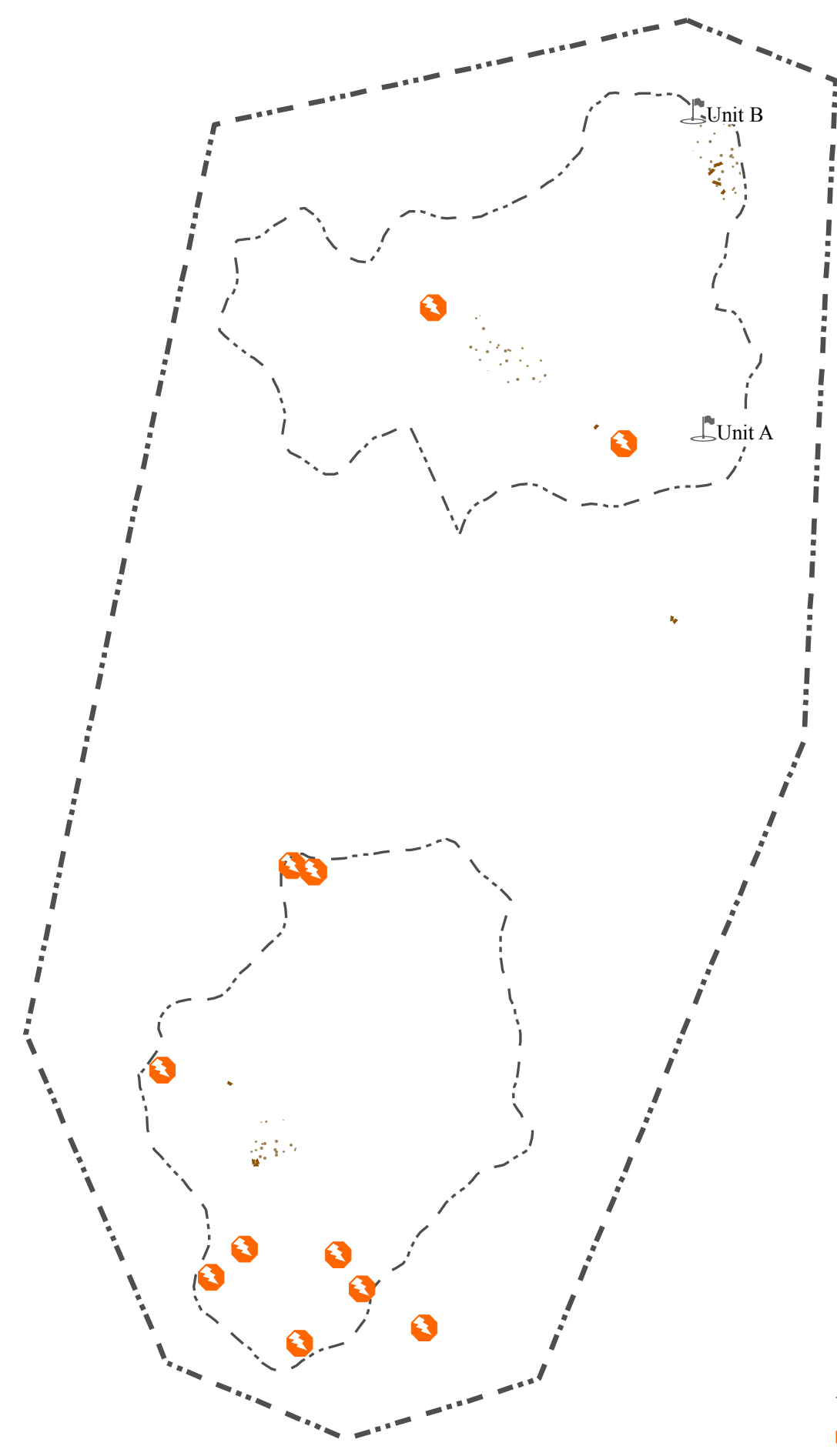

\& Collection Units

3 Fire Cracked Rock

Cist

Rubble

I Site Boundary

I MNA Site Boundary 


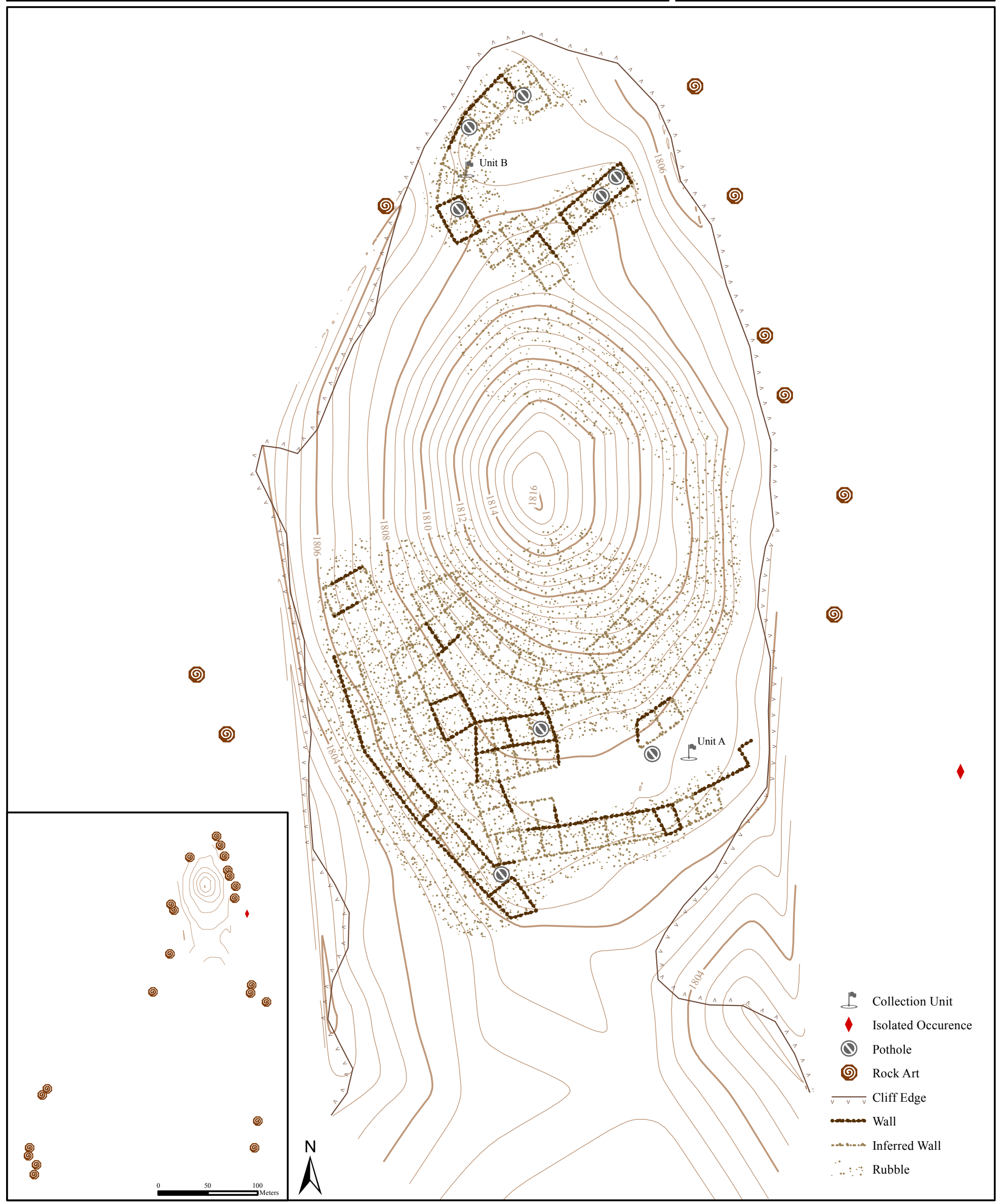




\section{Coal Mine Canyon \\ NA30505}

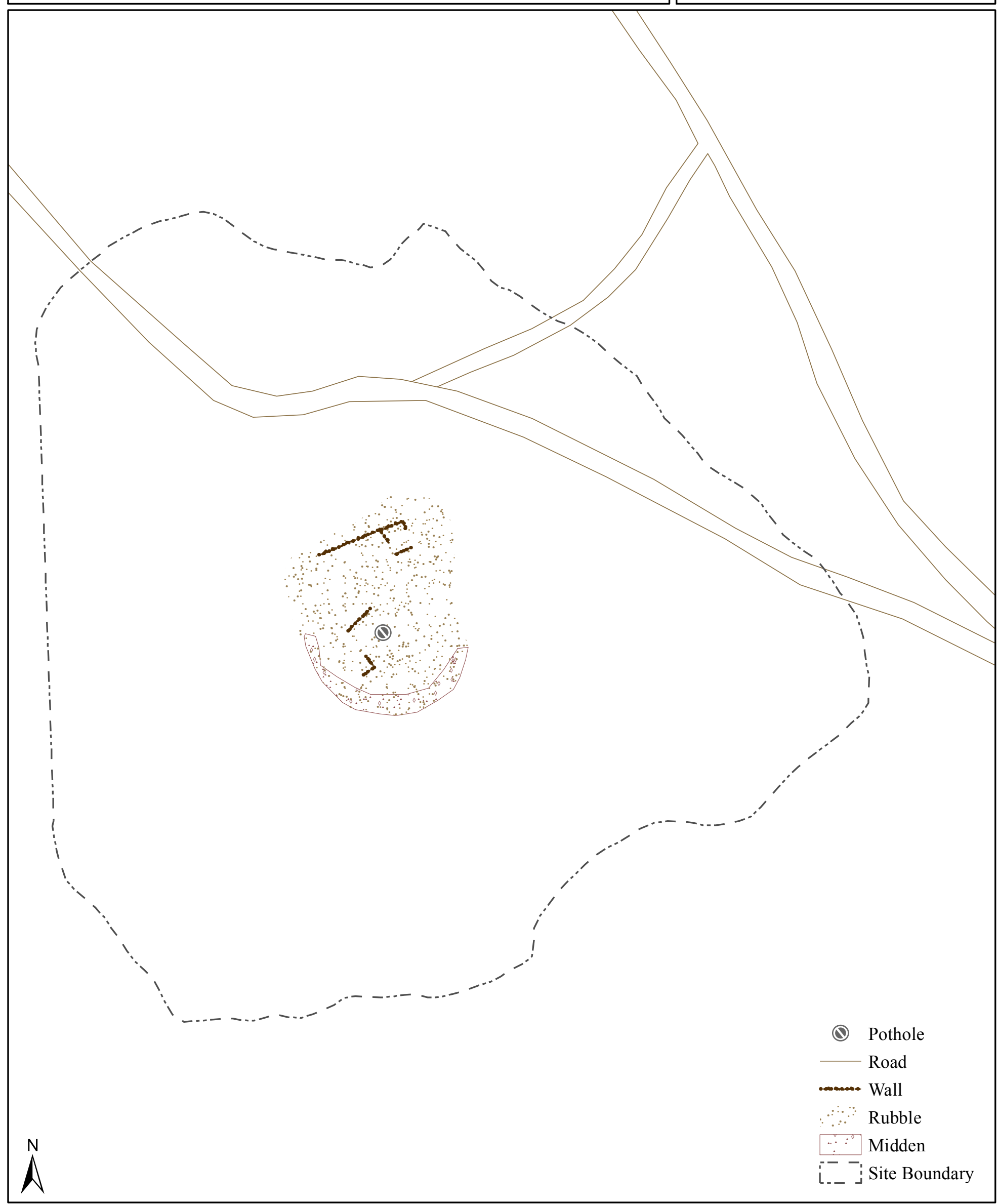




\section{Coyote Springs Survey}

NA30642; NA30643; NA3093

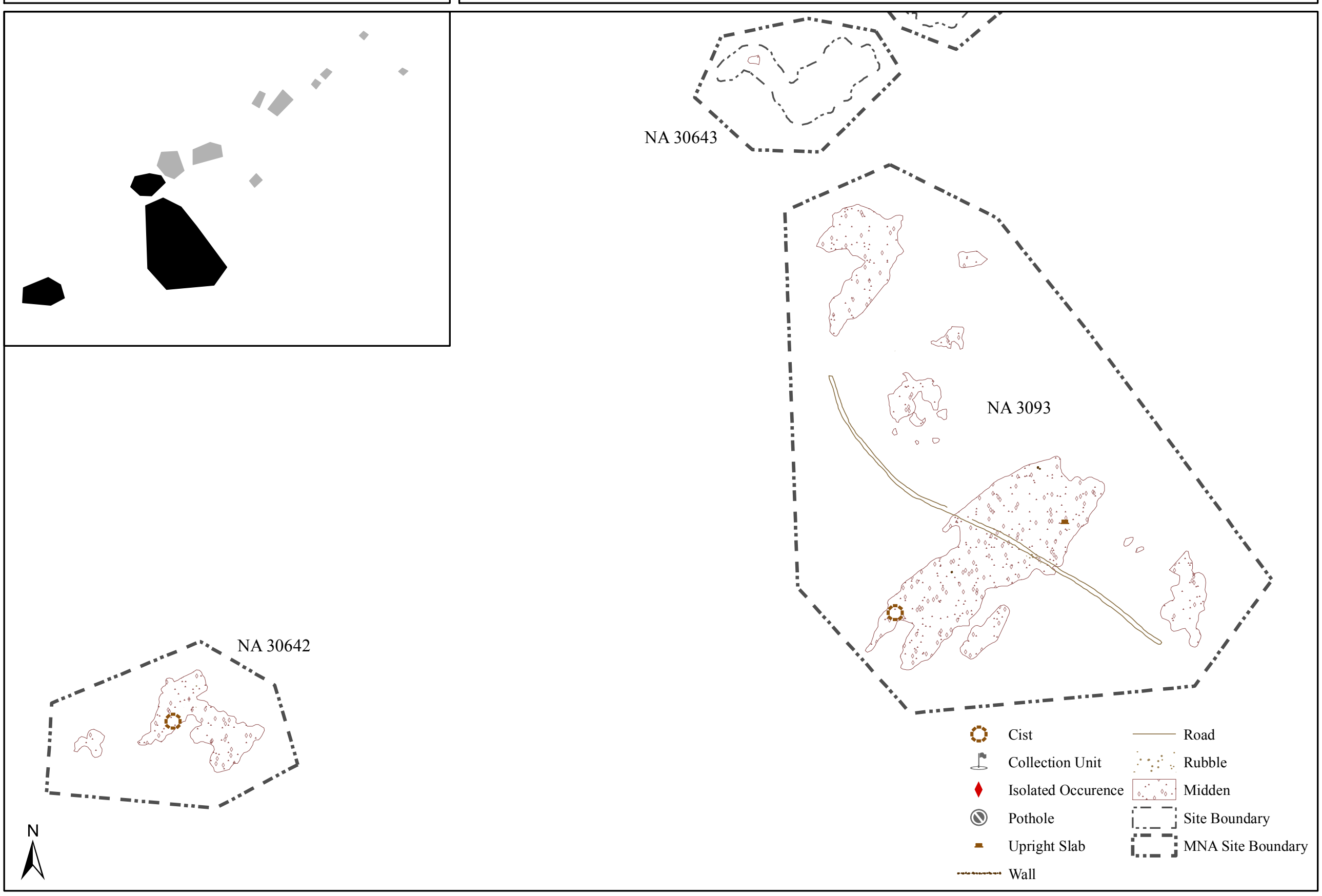

50 


\section{Coyote Springs Survey NA30643 - NA30650}

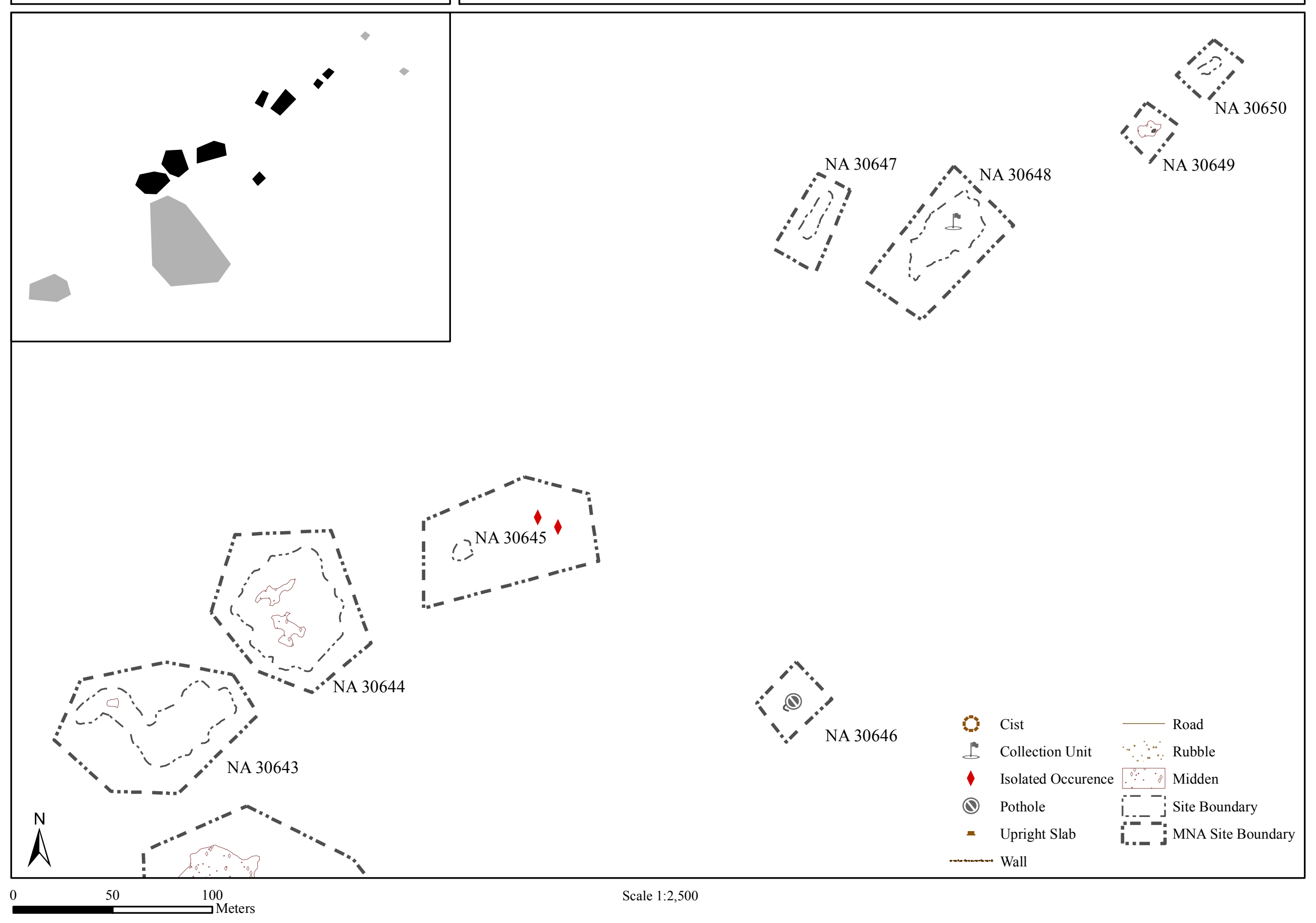




\section{Coyote Springs Survey NA30649 - NA30652}

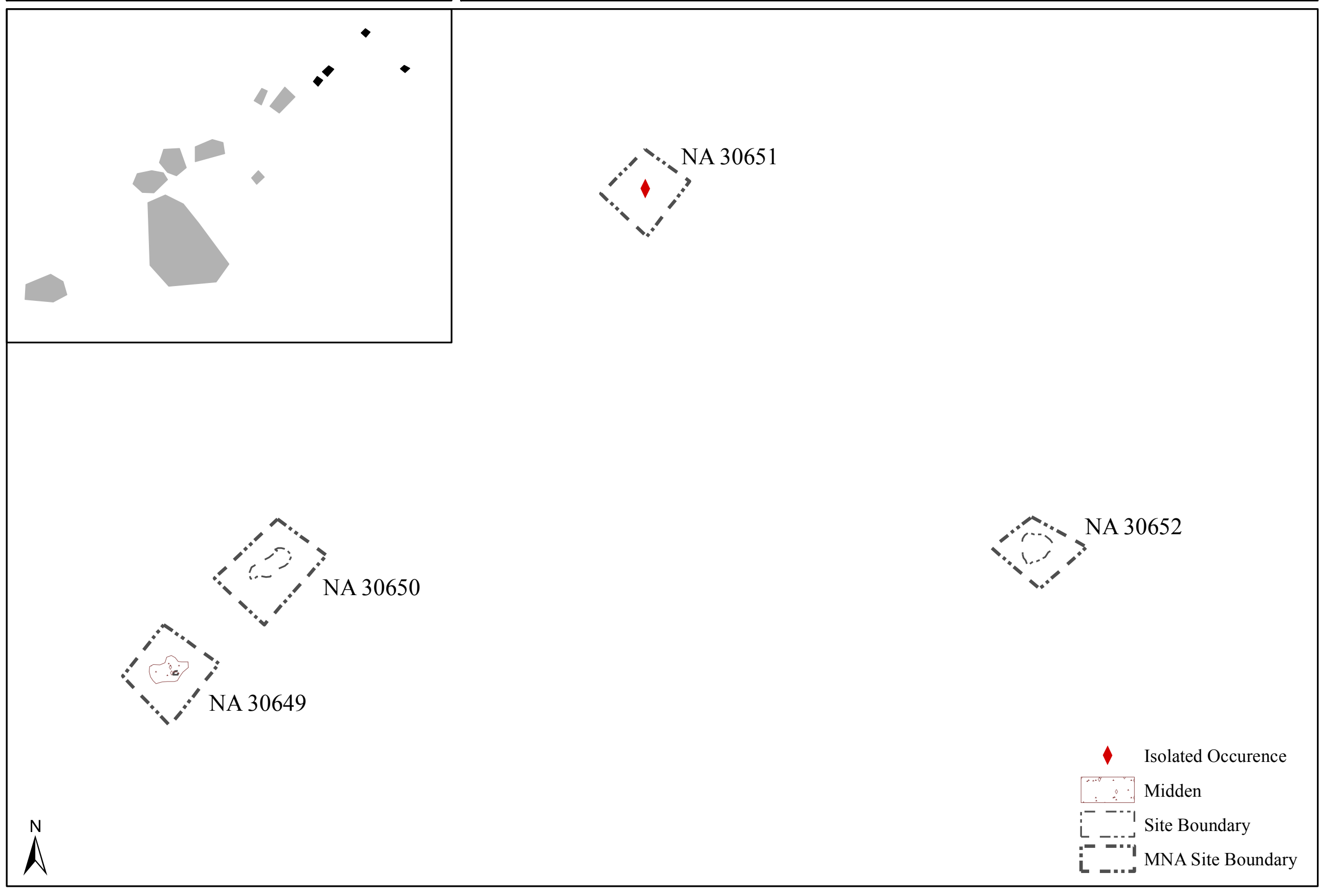




\section{Dawa Survey NA30473 - NA30475}

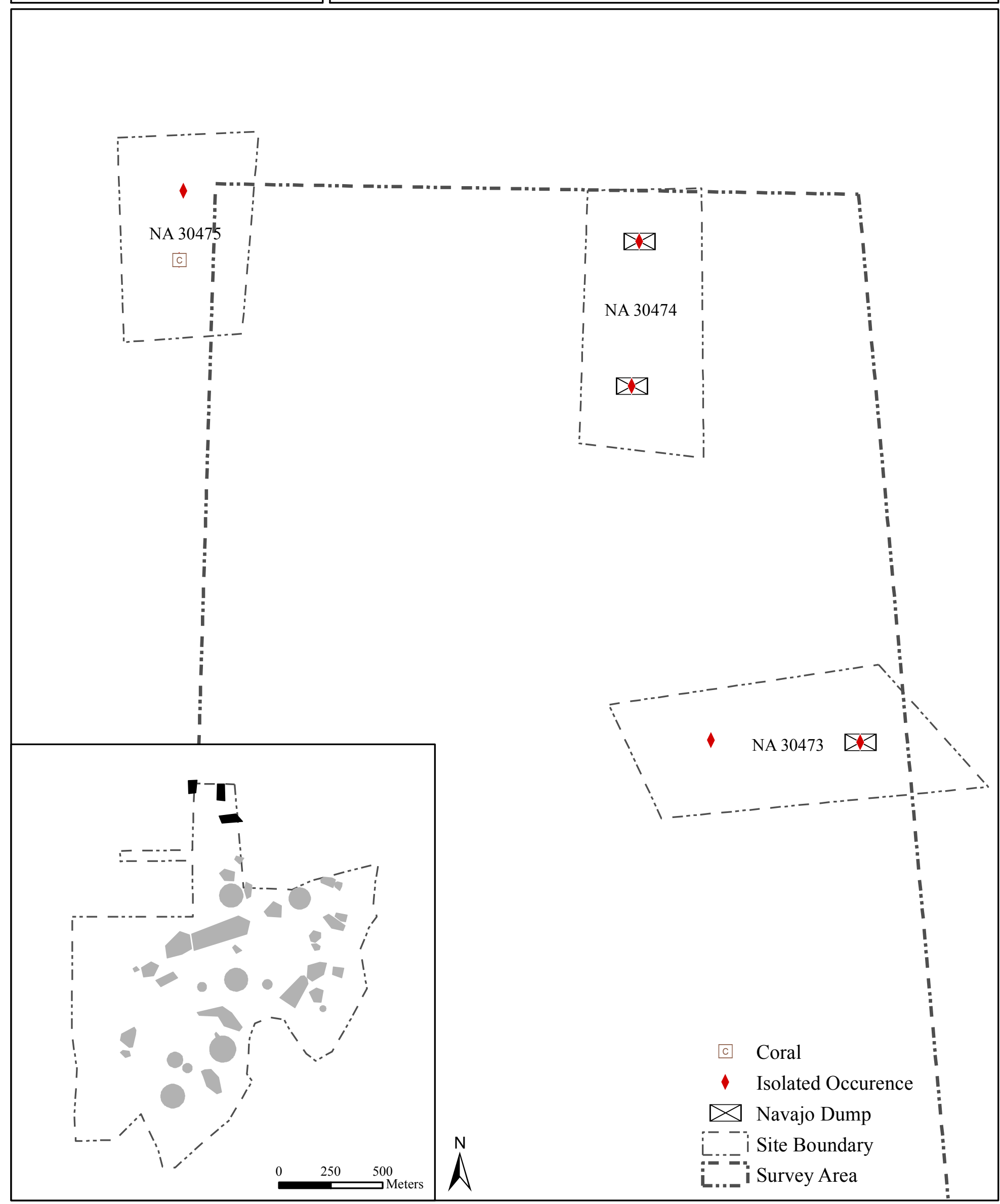




\section{Dawa Survey NA30469 - NA30472; NA30464; NA30462}

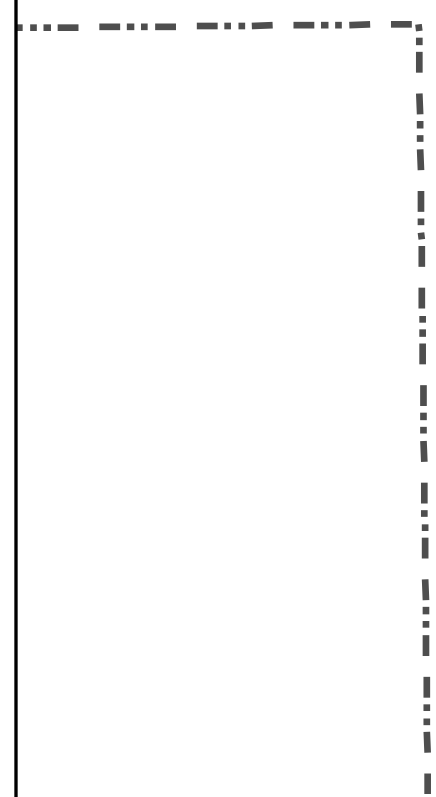

Collection Unit

3 Fire Cracked Rock

- Isolated Occurence

- Sweat Lodge

\& Shrine

Cist

L_- i Site Boundary

i. Survey Area
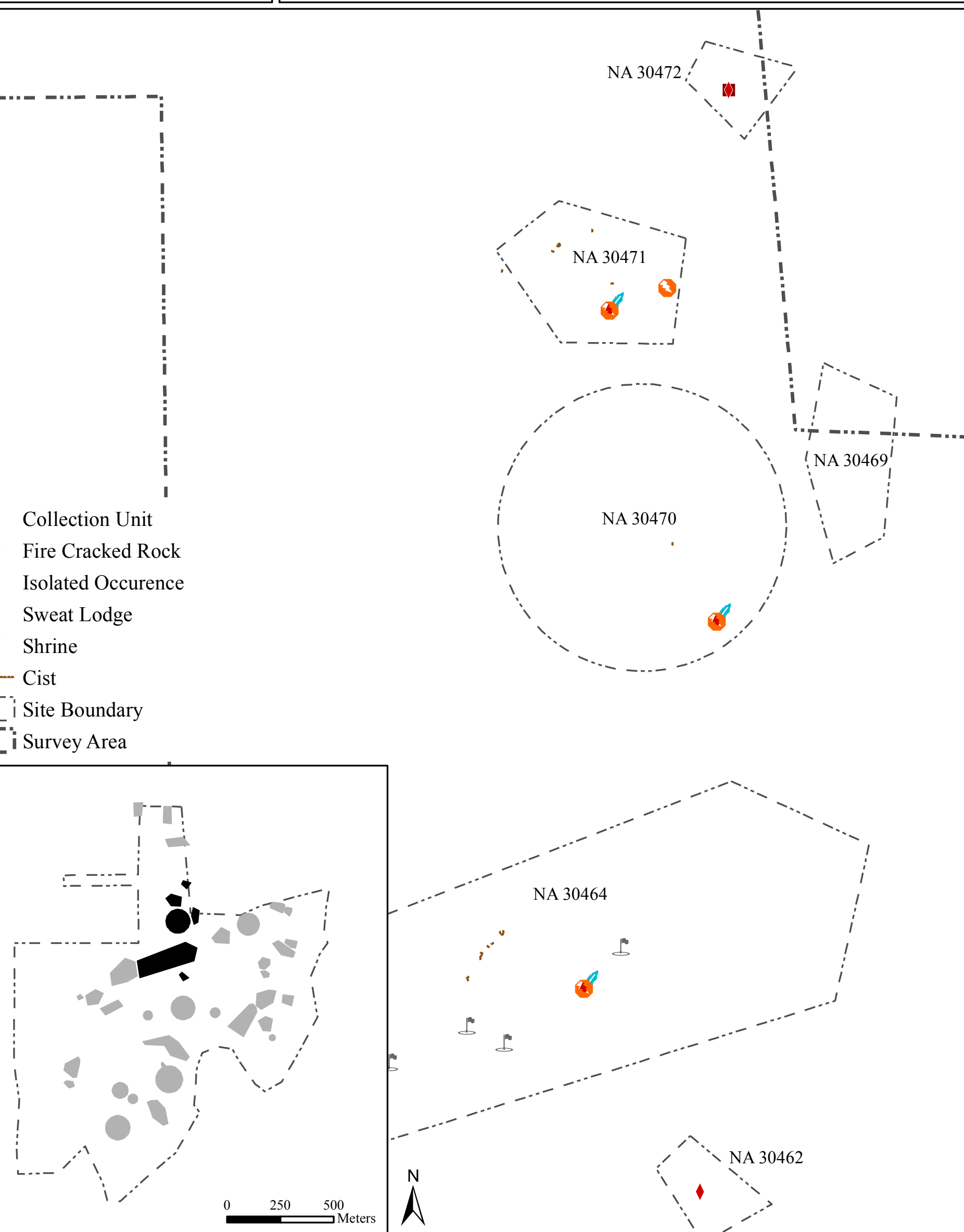


\section{Dawa Survey NA30453, NA30455 - NA30461; NA30463}

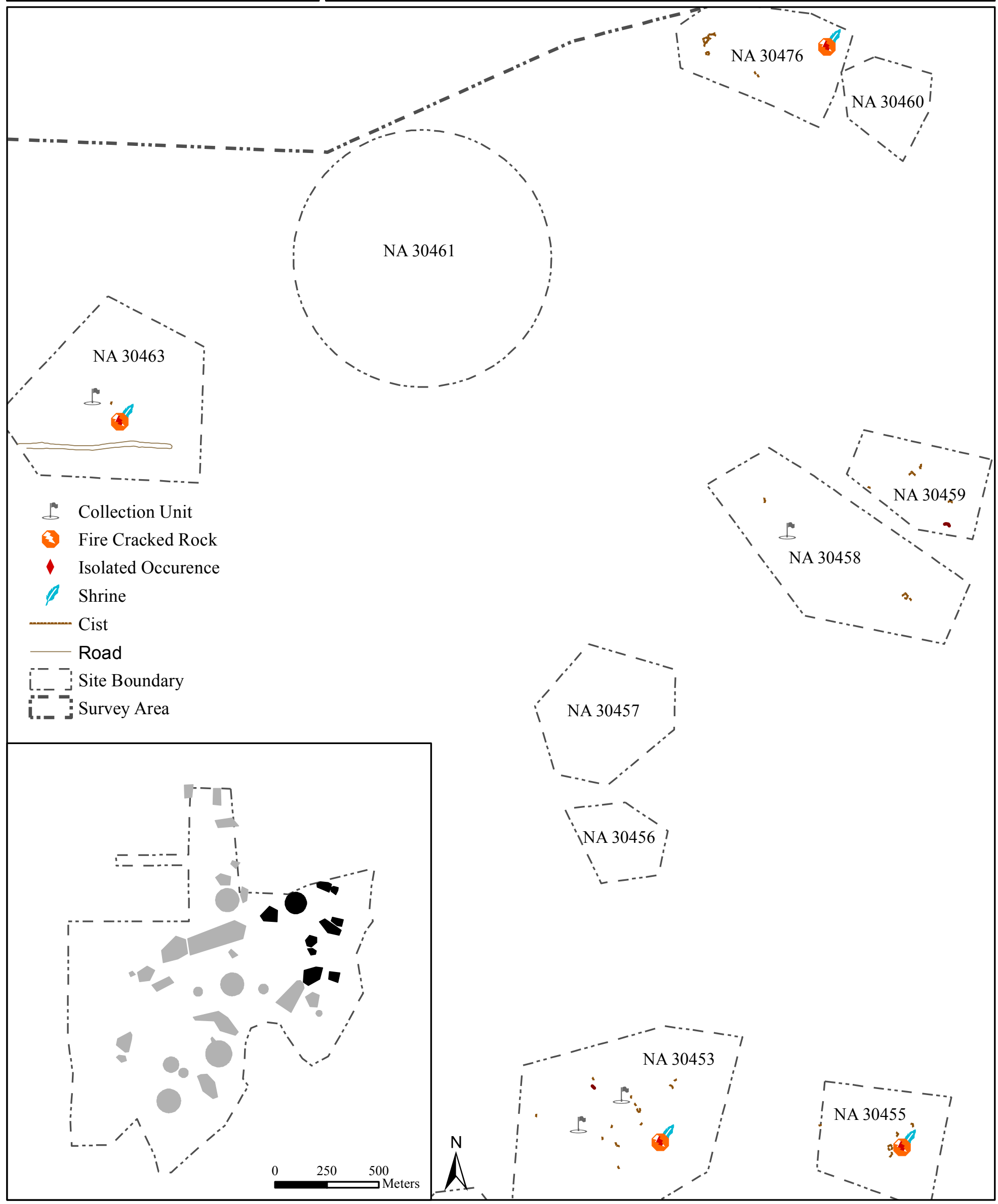




\section{Dawa Survey NA30446; NA30451 - NA30455}

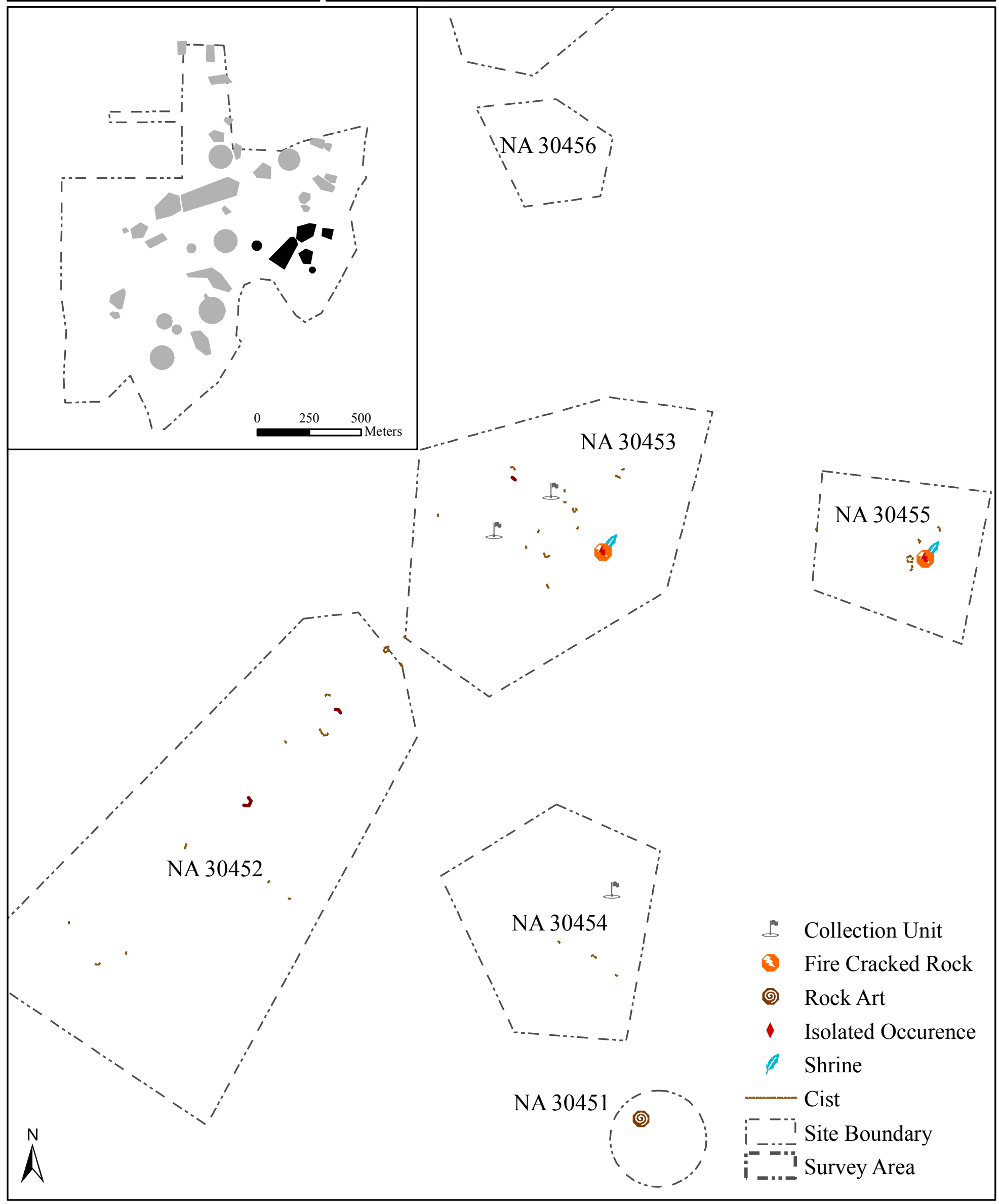




\section{Dawa Survey NA30265, NA30445, NA30449, NA30450}
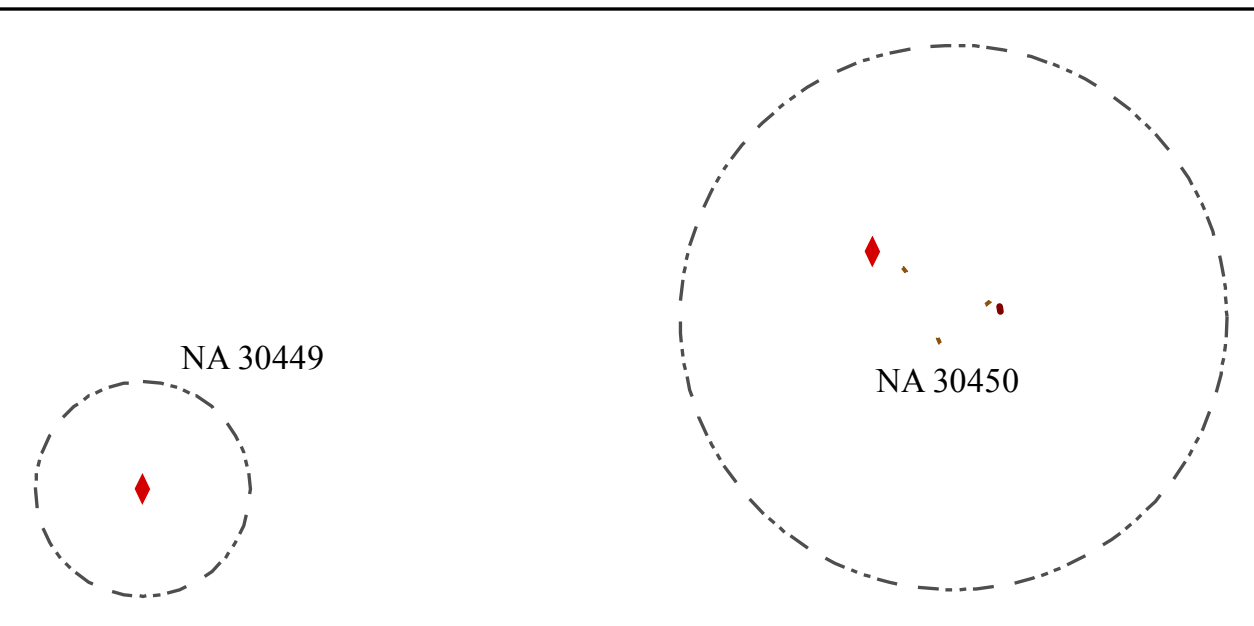

D Collection Unit

- Isolated Occurence

(2) Rock Art

- Cist

i Site Boundary

i_.._ Survey Area 


\section{Dawa Survey NA30465 - NA30468}

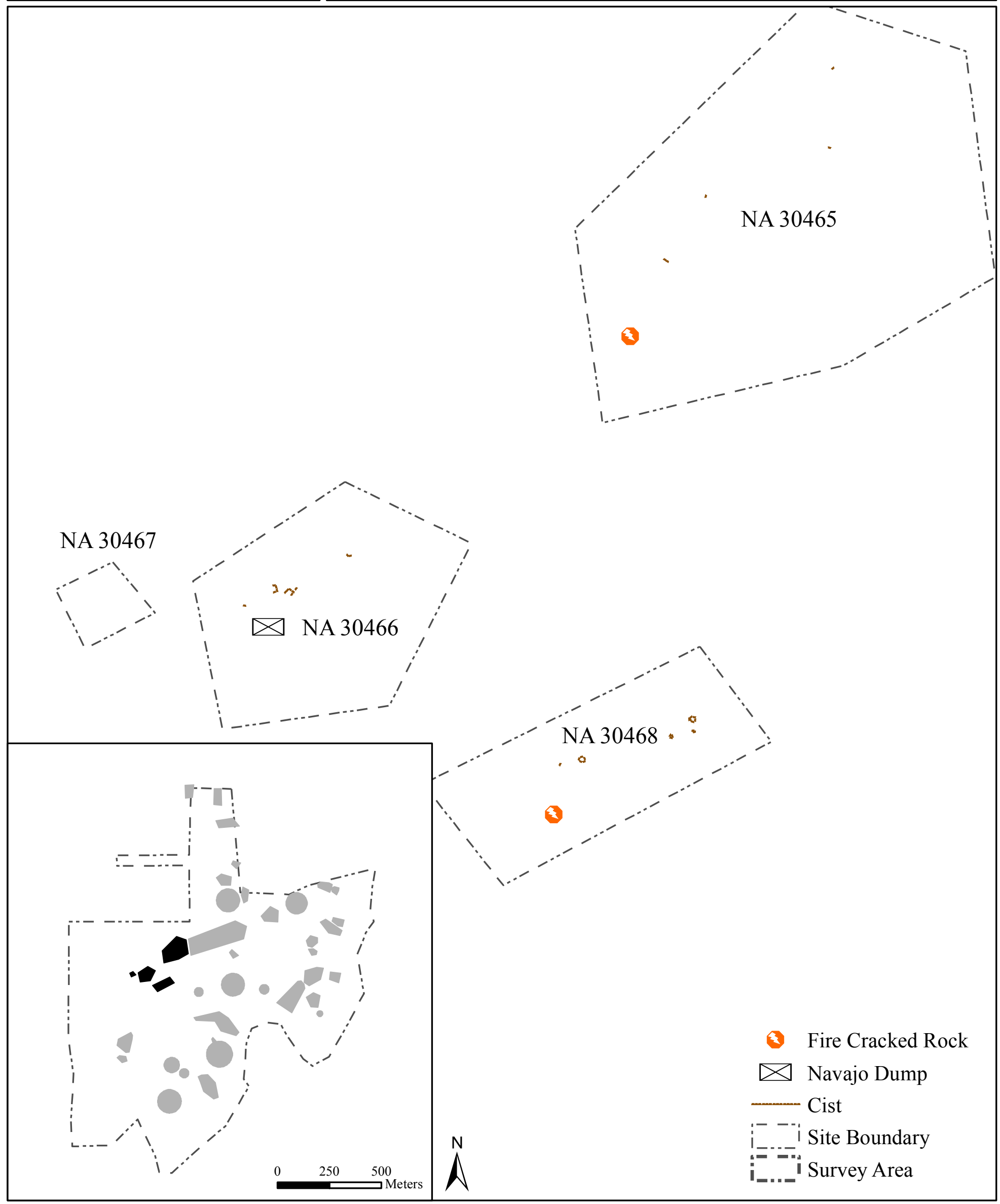




\section{Dawa Survey NA30261 - NA30264; NA30447; NA30448}

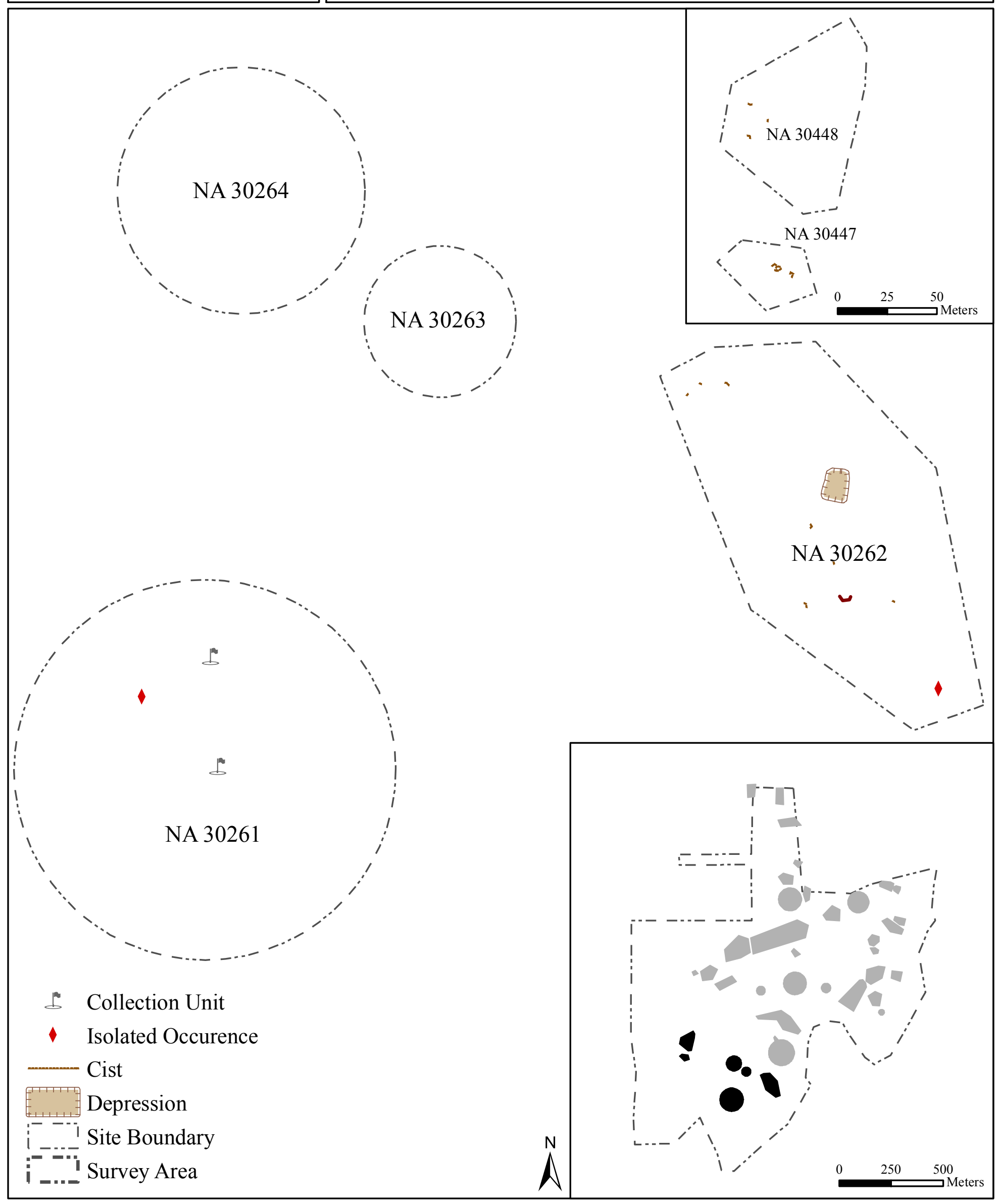




\section{\begin{tabular}{l||l} 
Dawake Survey & NA30653 - NA30659
\end{tabular}}
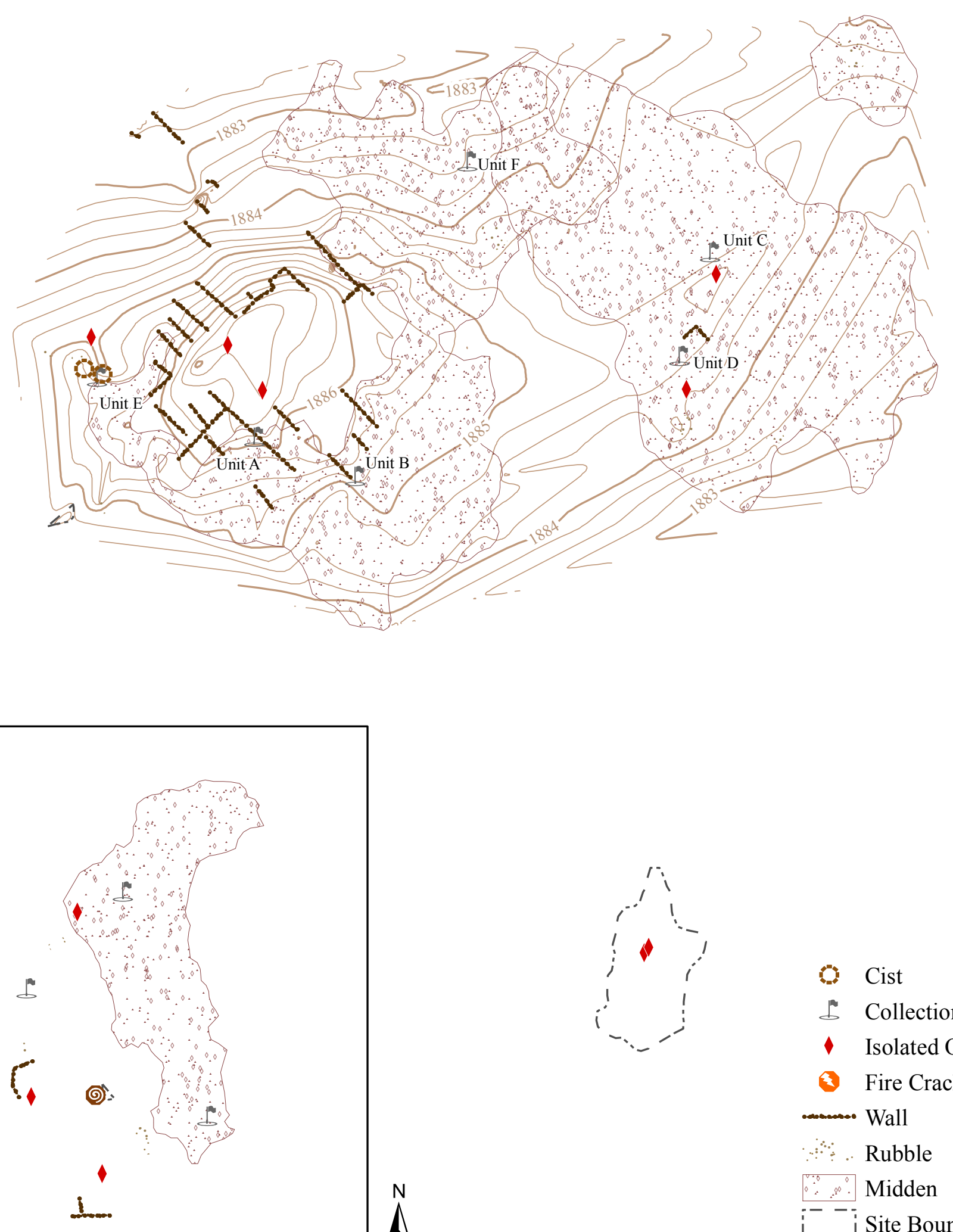

\begin{tabular}{|c|c|}
\hline 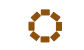 & Cist \\
\hline $\mathcal{L}$ & Collection Unit \\
\hline$v$ & Isolated Occurence \\
\hline 3 & Fire Cracked Rock \\
\hline & Wall \\
\hline & Rubble \\
\hline & Midden \\
\hline & Site Boundary \\
\hline
\end{tabular}




\section{First Mesa Survey}

\section{NA30171; NA30488; NA30489}

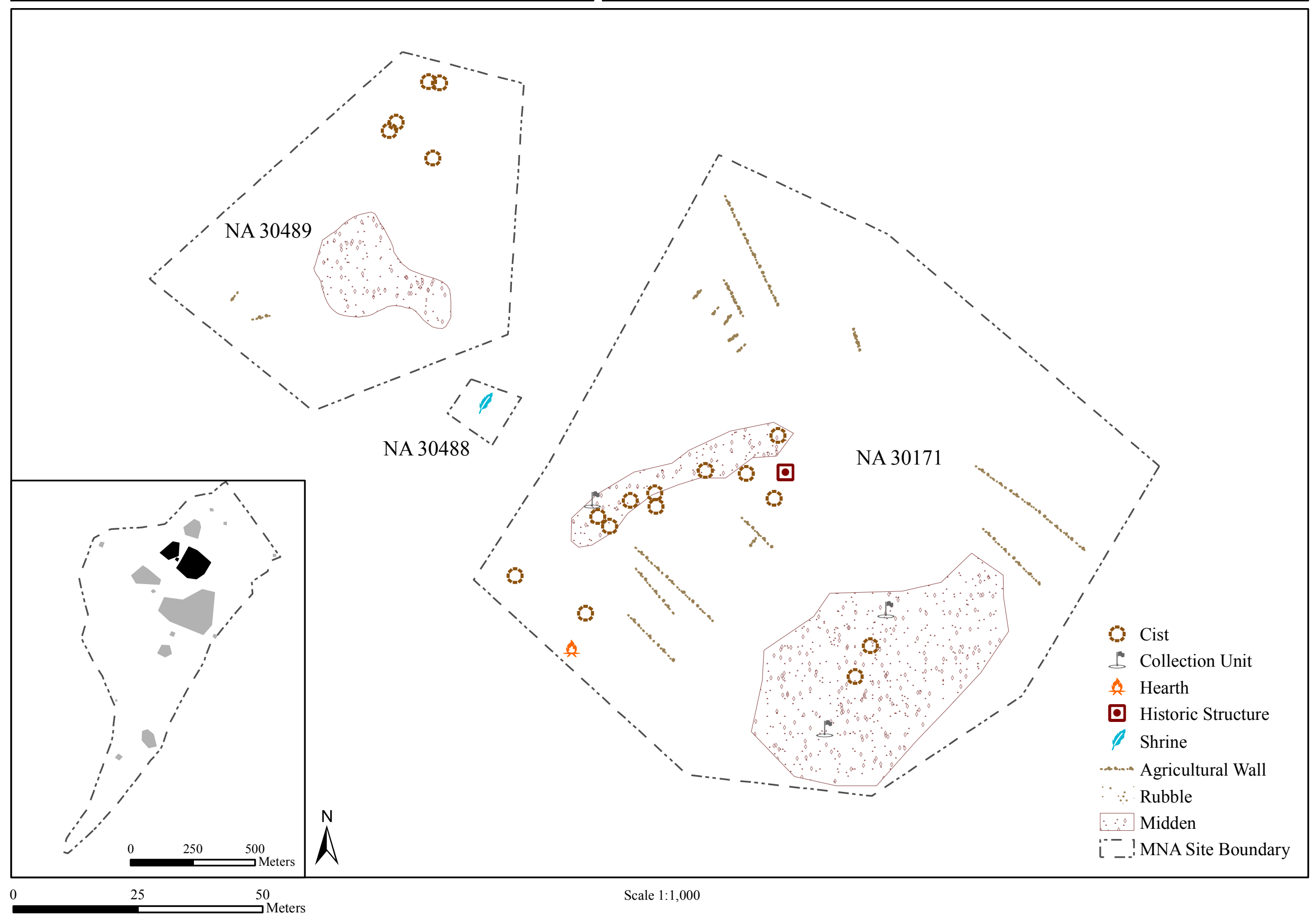




\section{First Mesa Survey}

NA30490; NA30491; NA30492

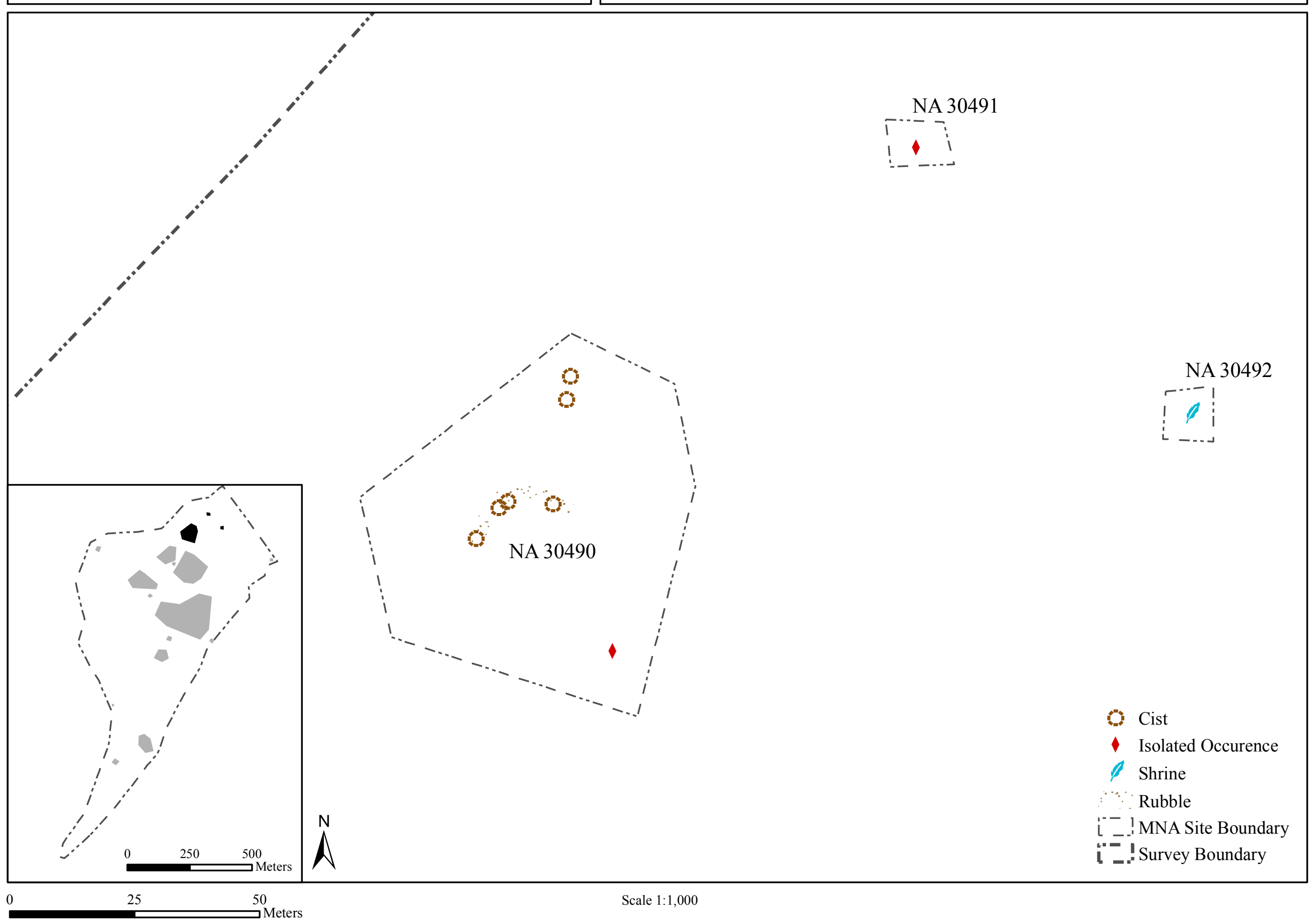




\section{First Mesa Survey \\ NA30481; NA30482; NA30483; NA30485: NA30486}

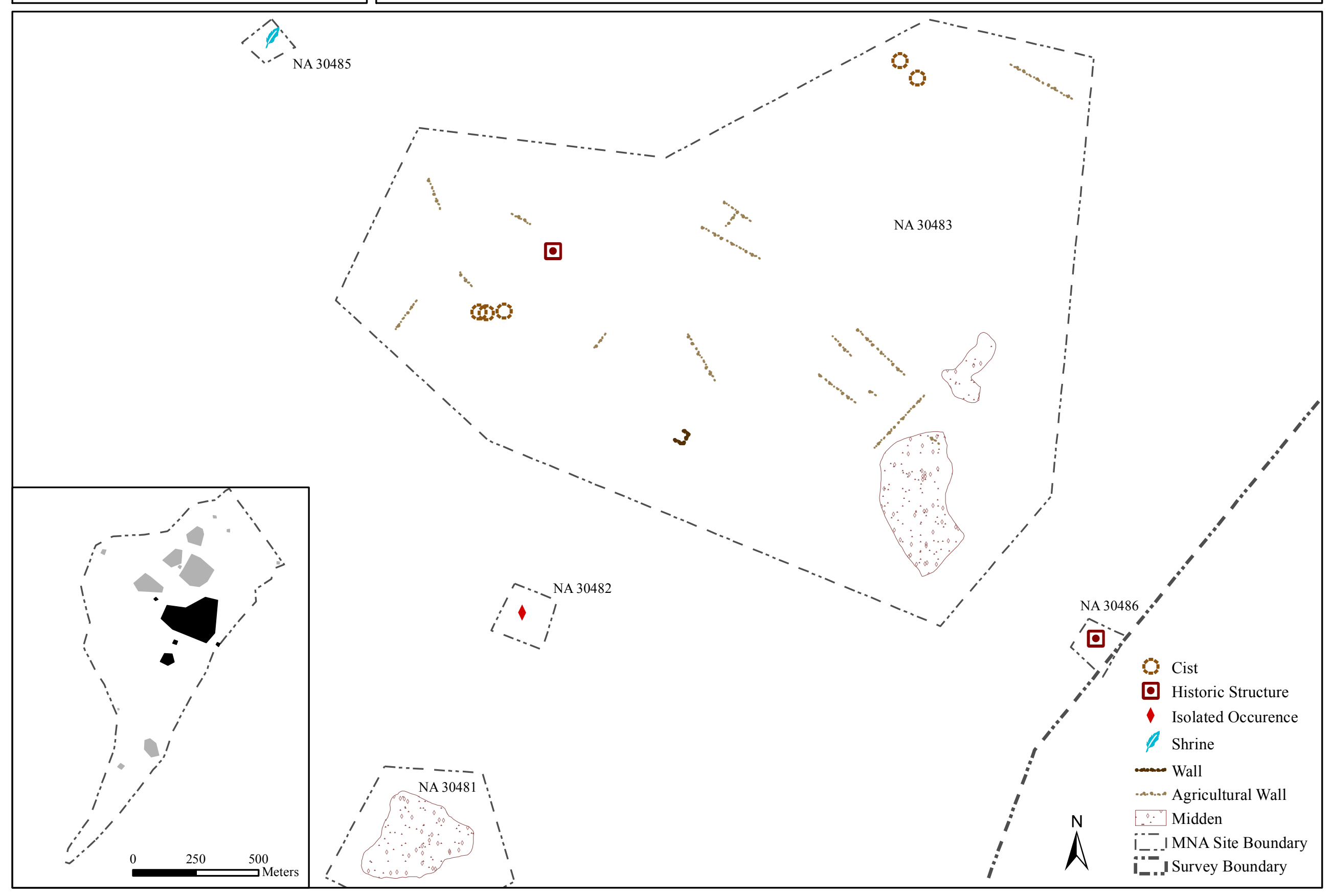




\section{First Mesa Survey}

NA30480; NA30494; NA30495

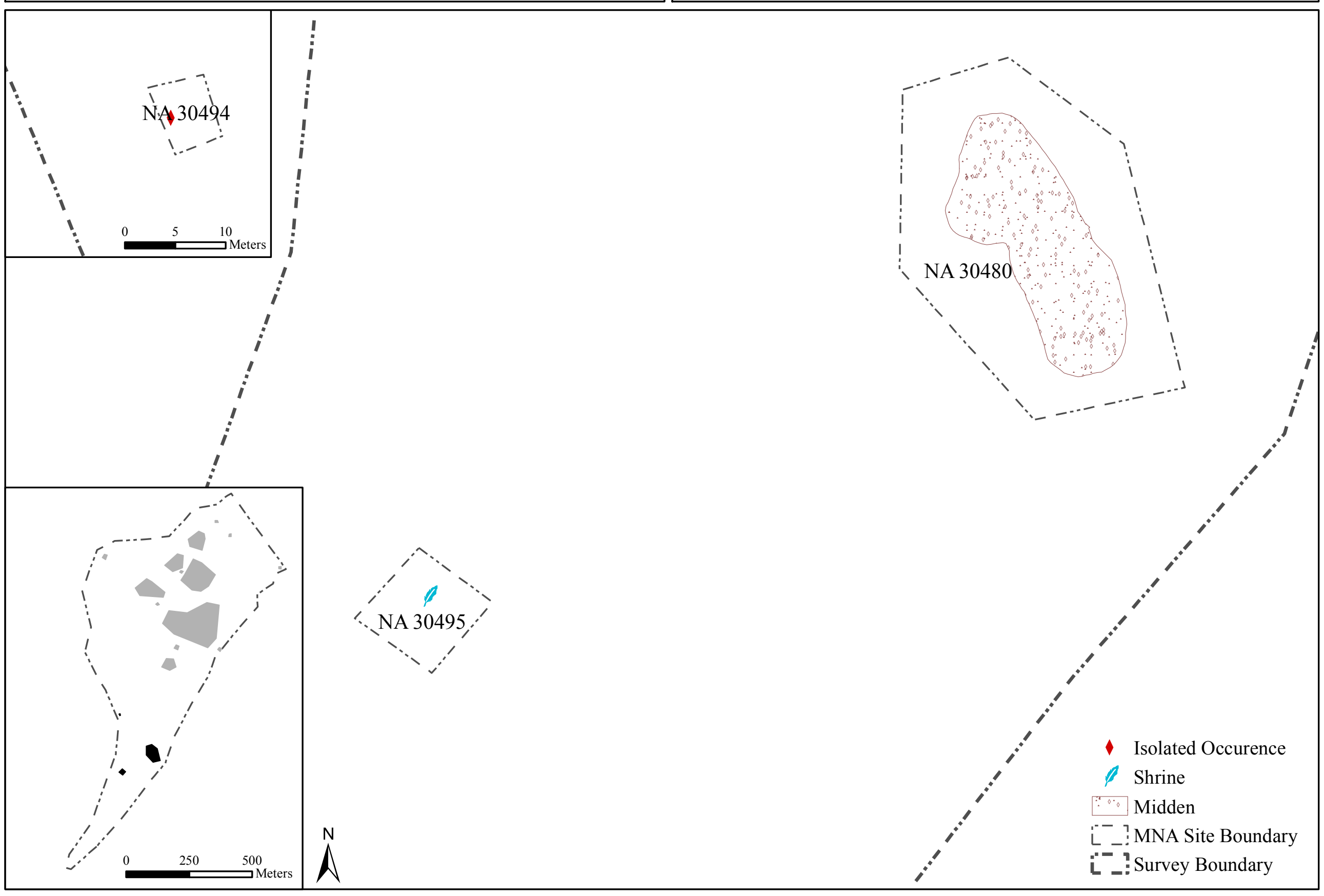




\section{First Mesa Survey}

NA30487; NA30484; NA30485

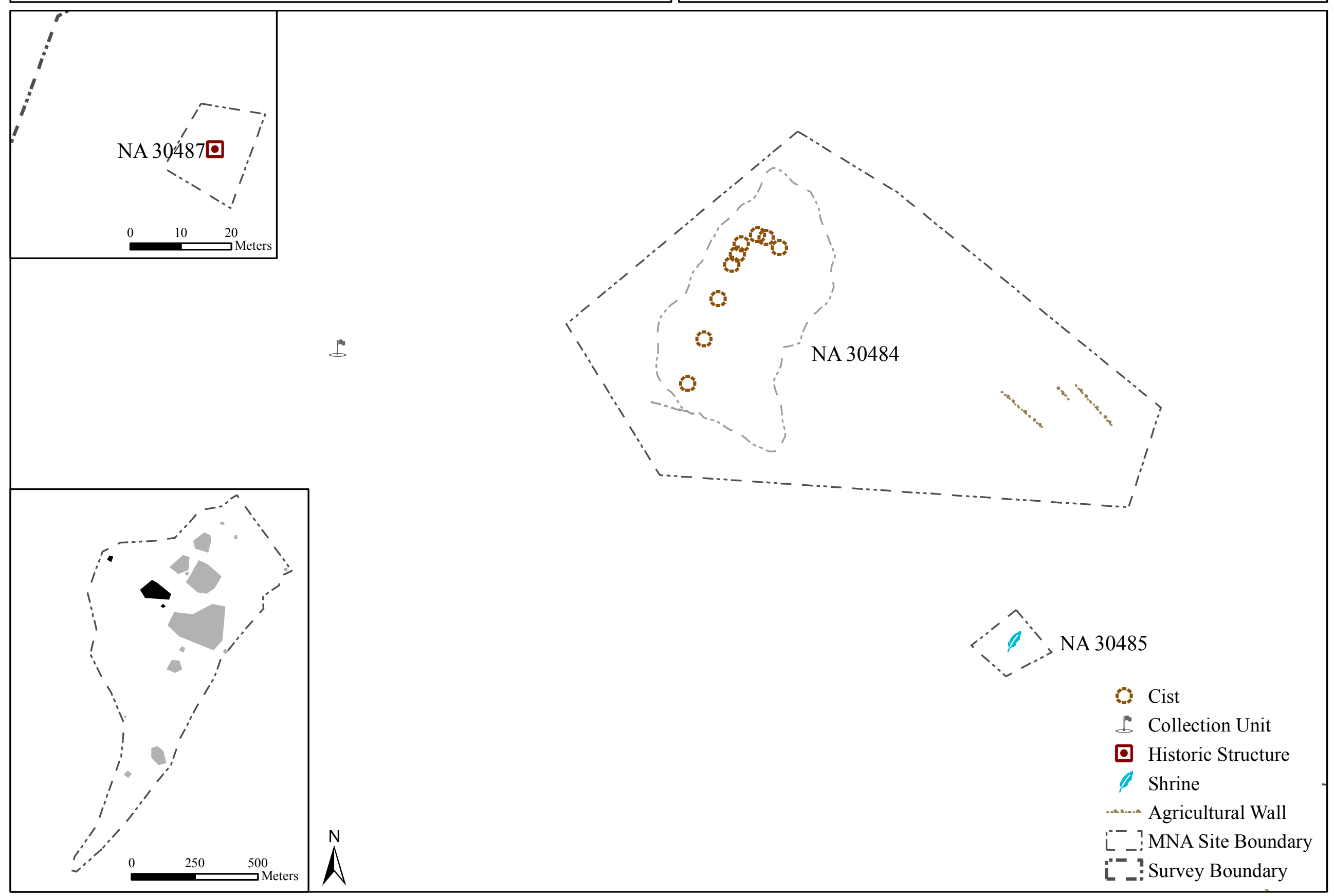




\section{Giant Chair}

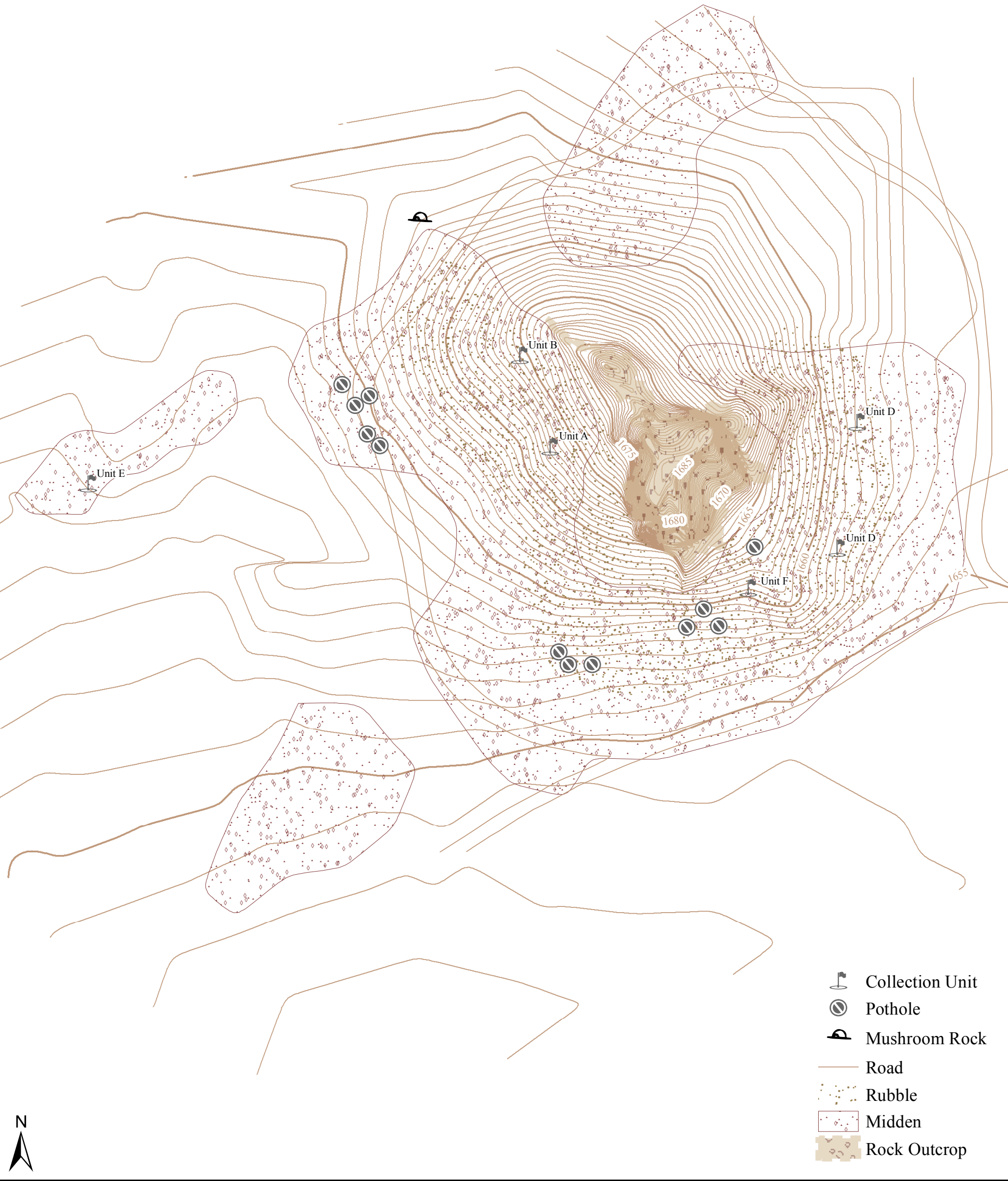




\section{Hukovi}

NA844

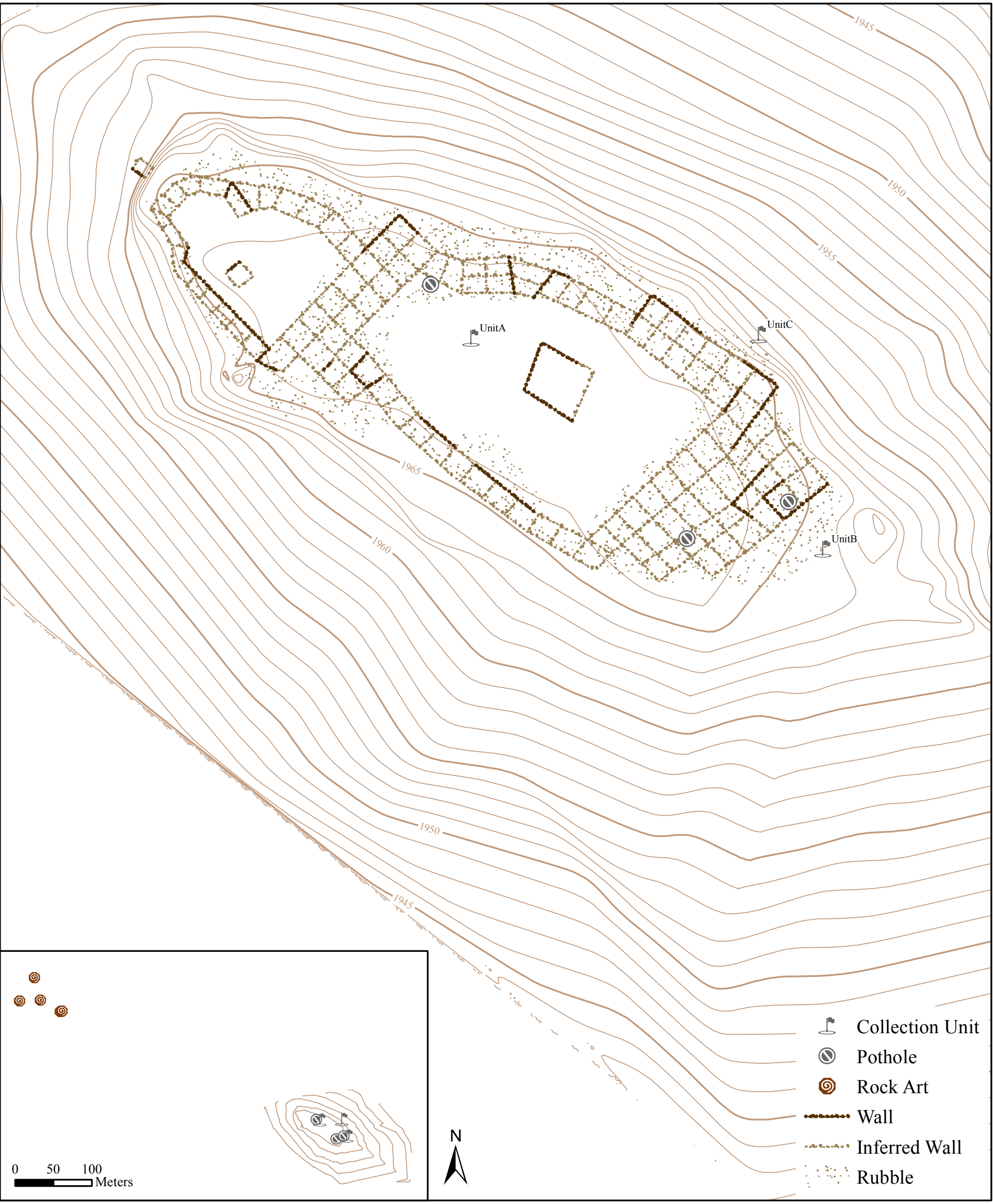




\section{Huvatuqwa \\ NA3095; NA30665; NA30666}

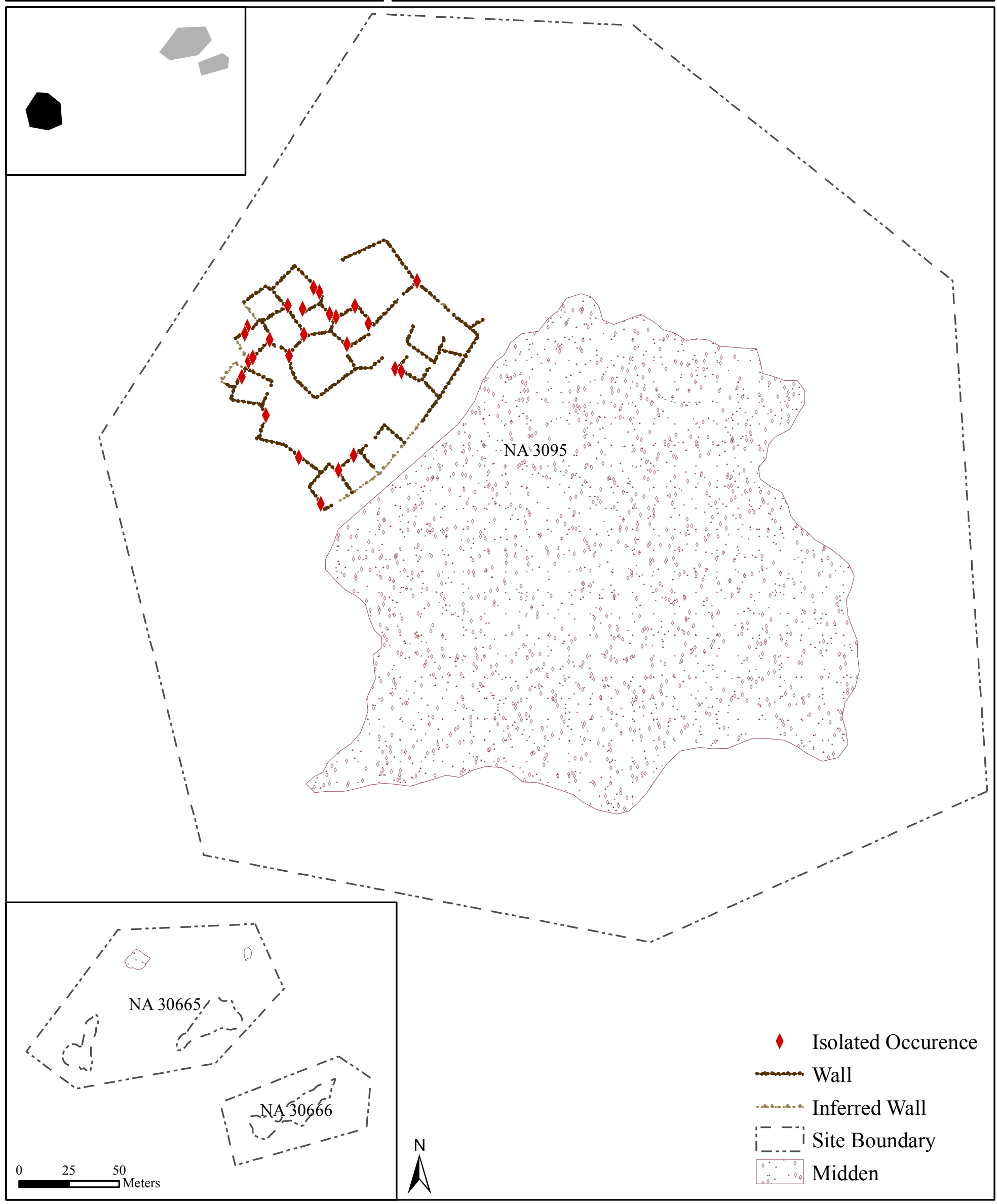




\section{Kawayka'a}

NA1001

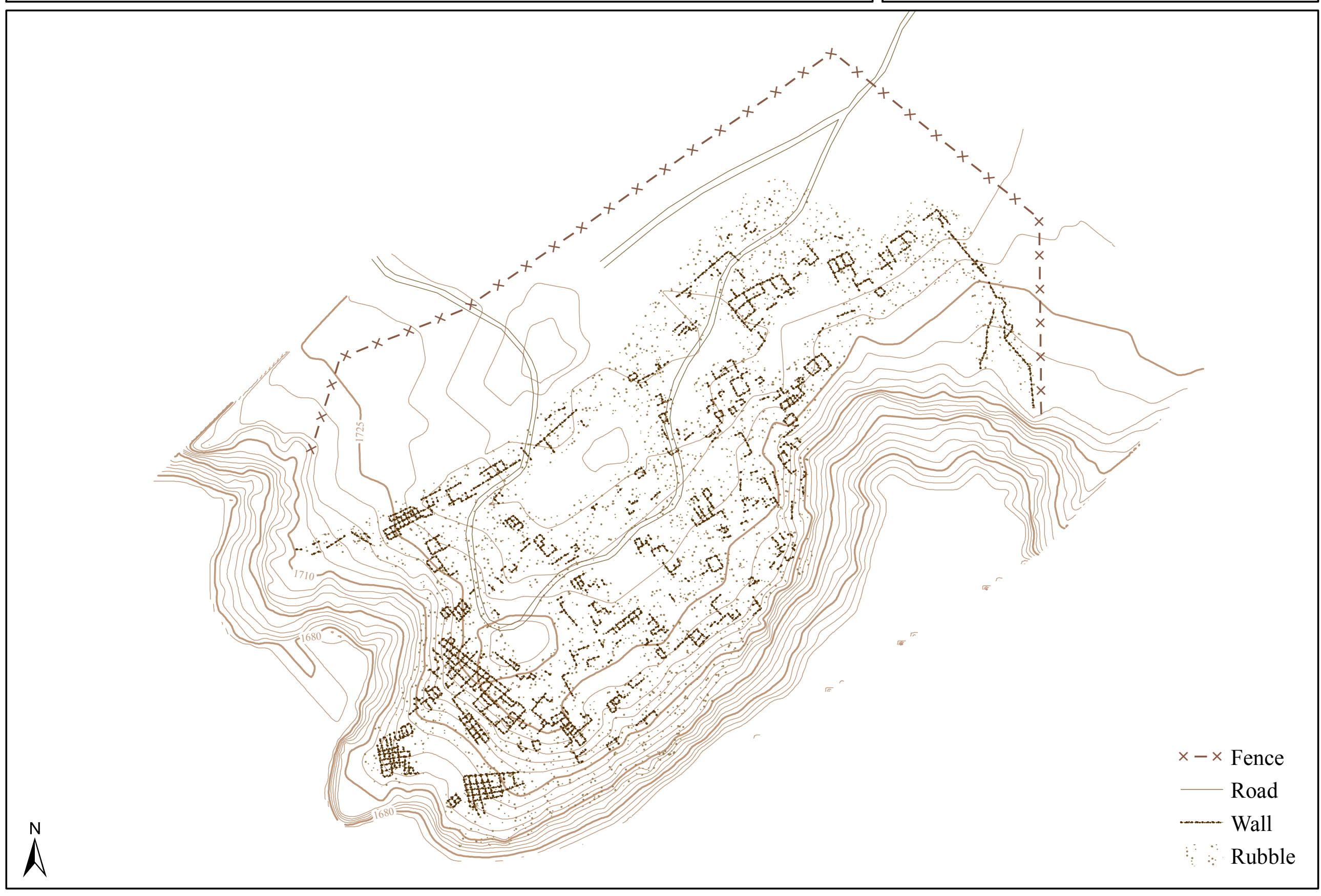




\section{Kisak'ovi}

NA813

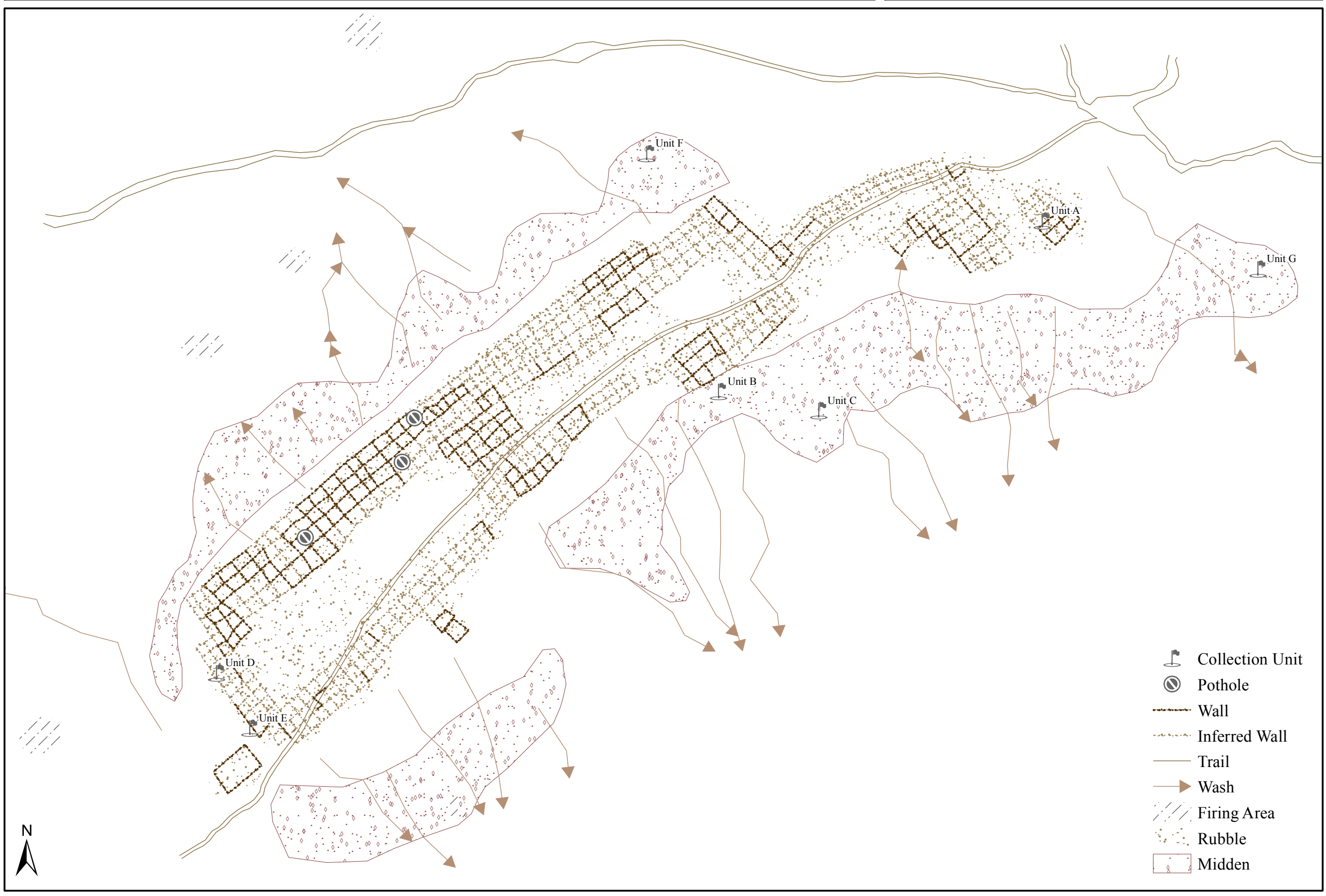




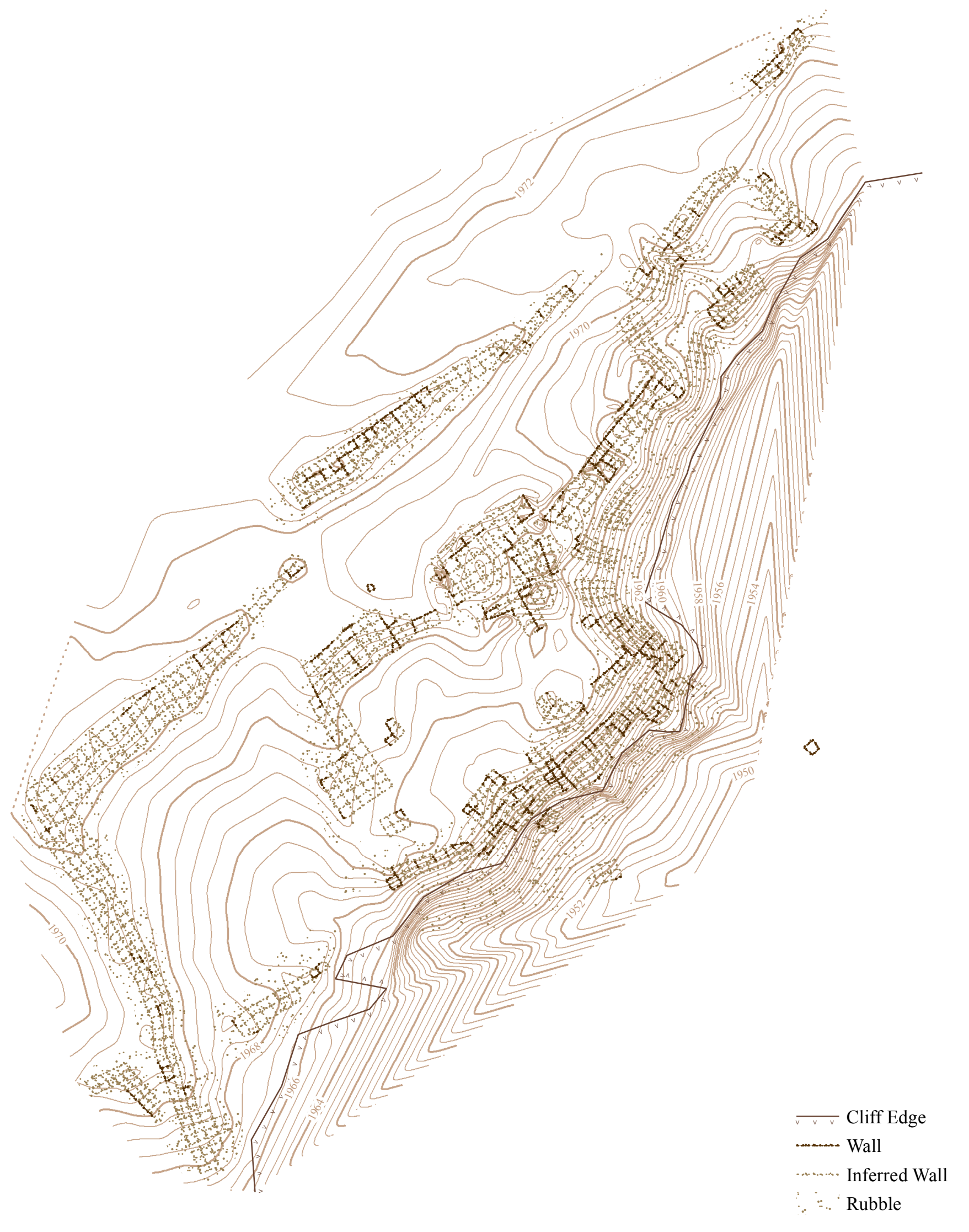

i 


\section{Kokotsomo}






\section{Lemeva \\ NA1707}

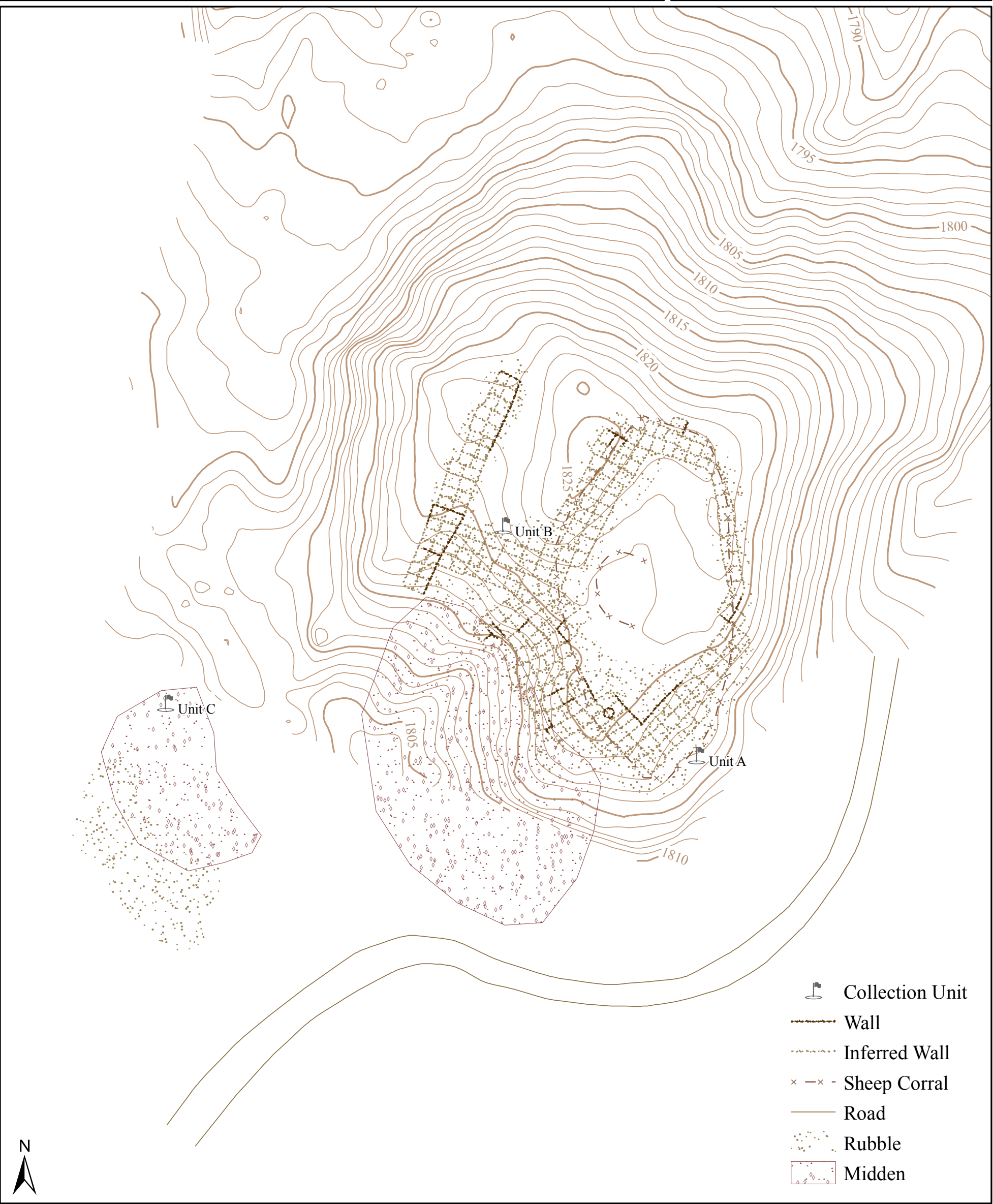




\section{Lolkangtuuyqa \\ NA1049}

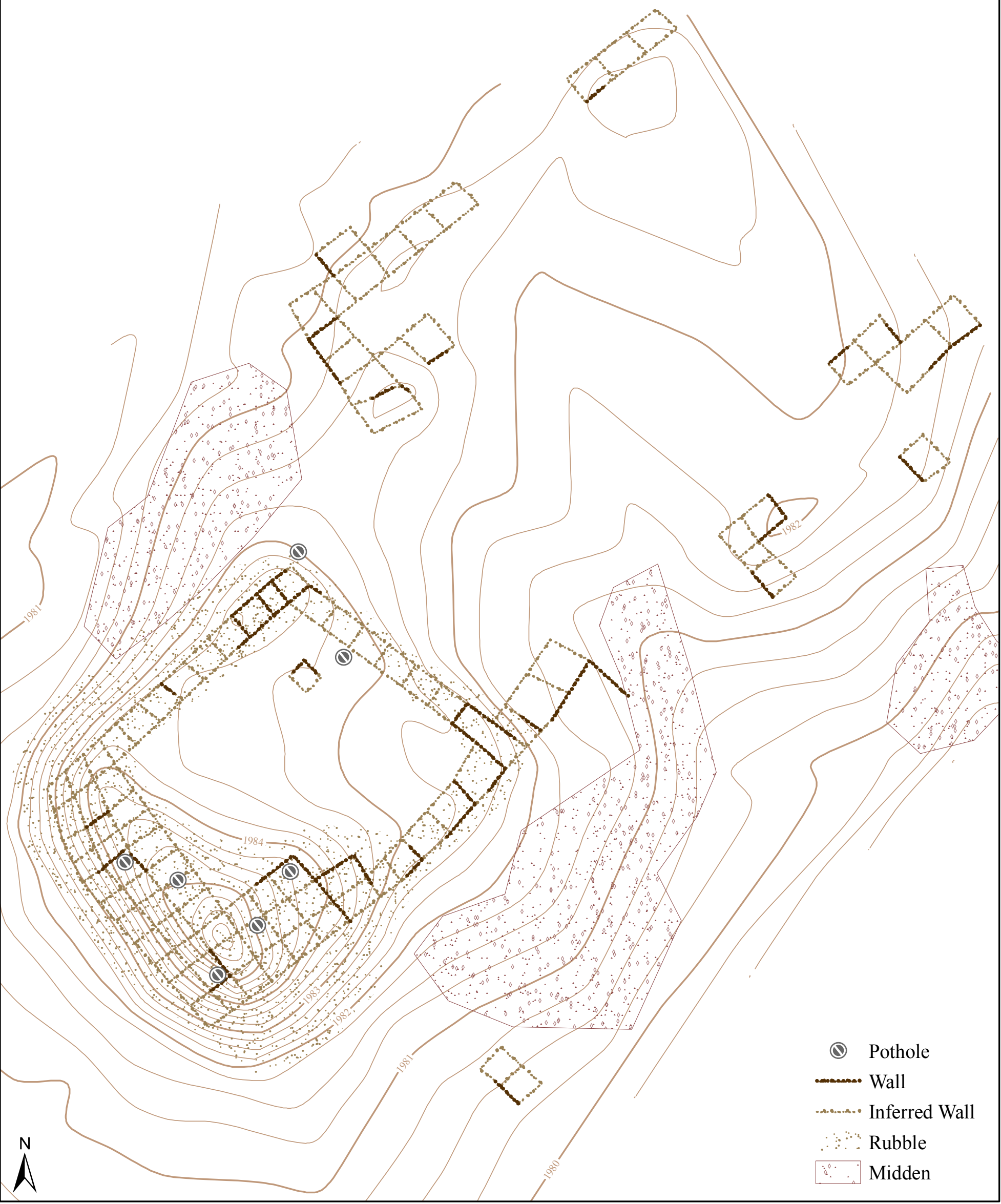




\section{Mosiptanga}

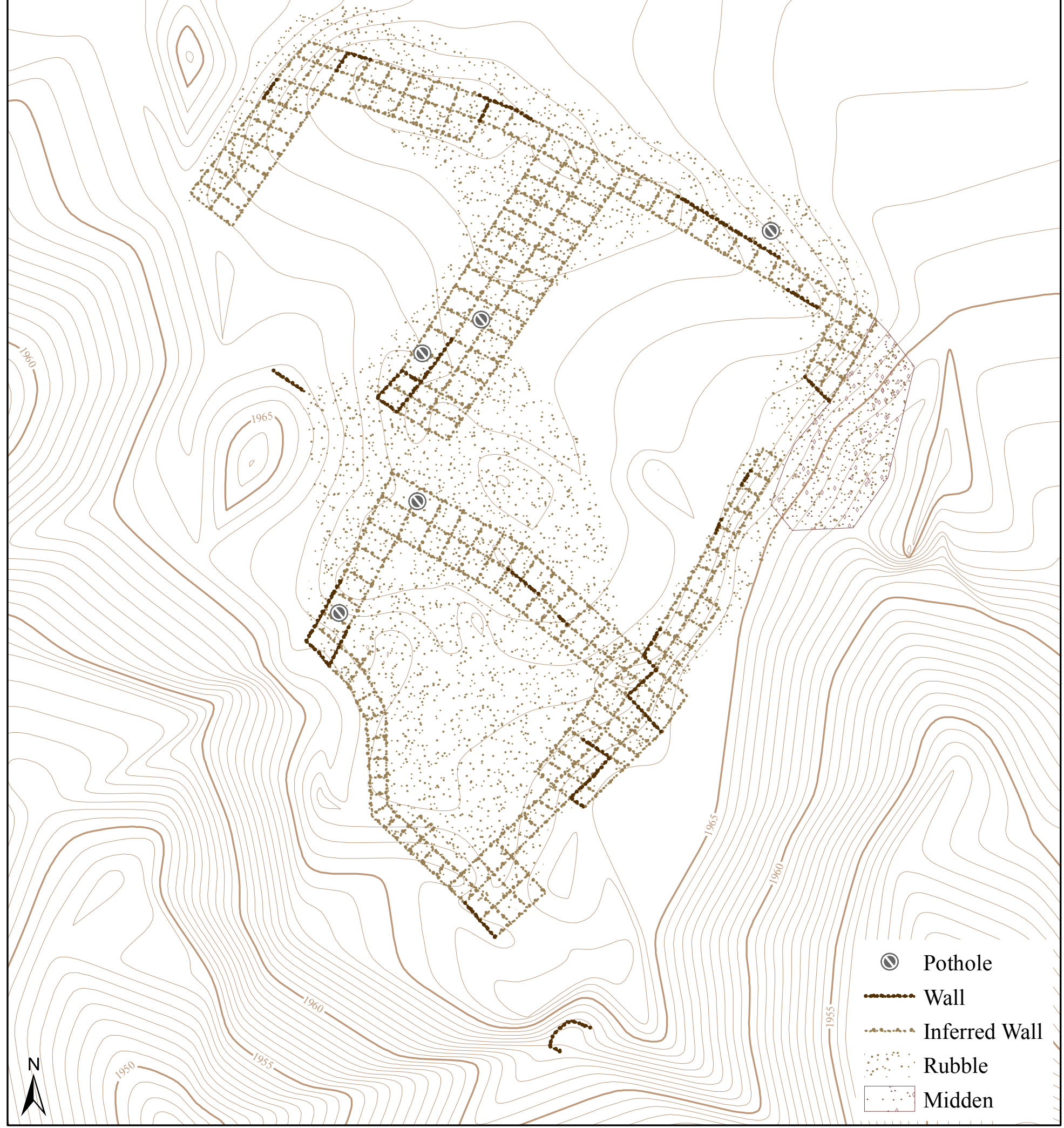




\section{Mungyaovi Survey}

NA30562

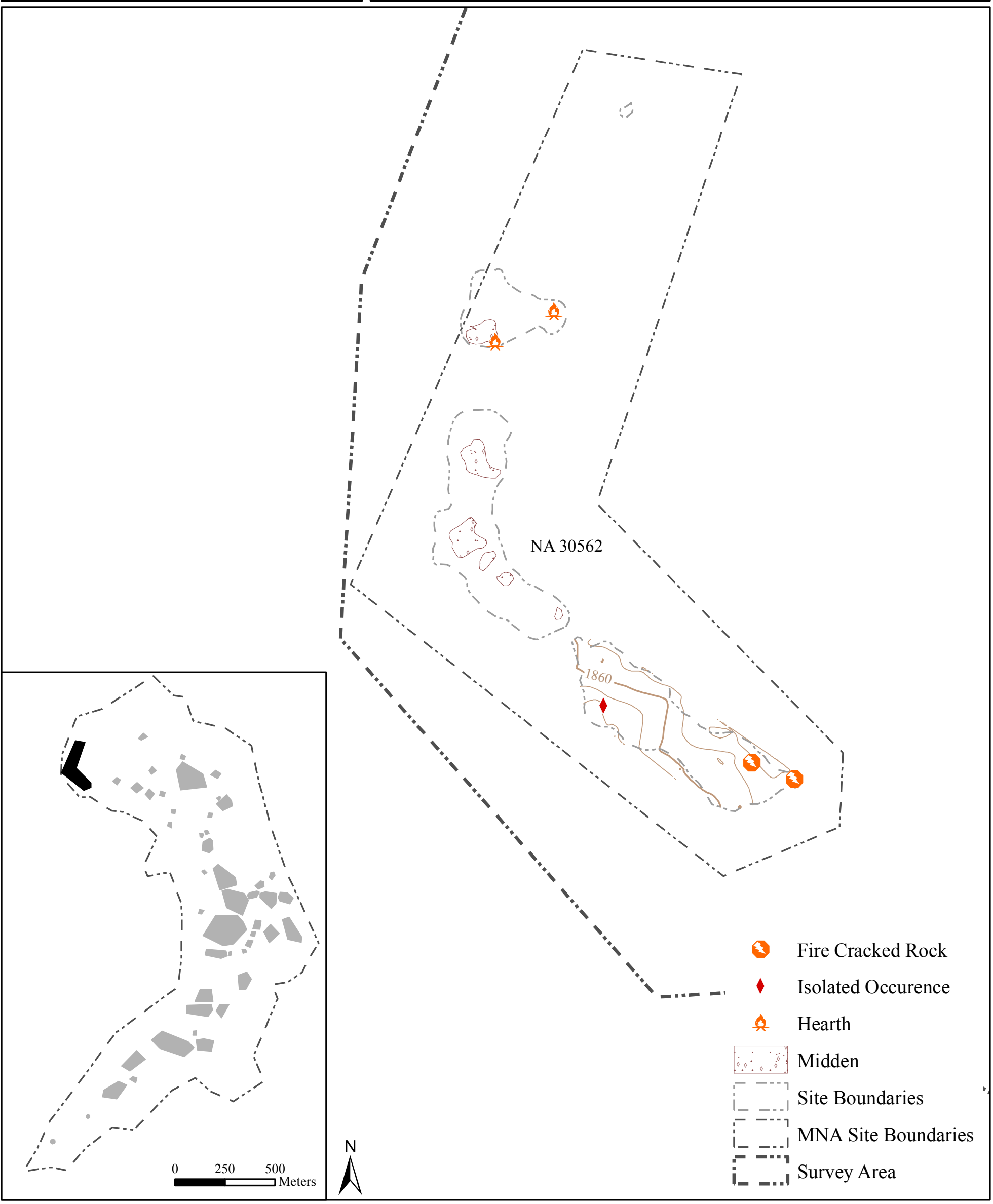




\section{Mungyaovi Survey}

\section{NA30556 - NA30560}

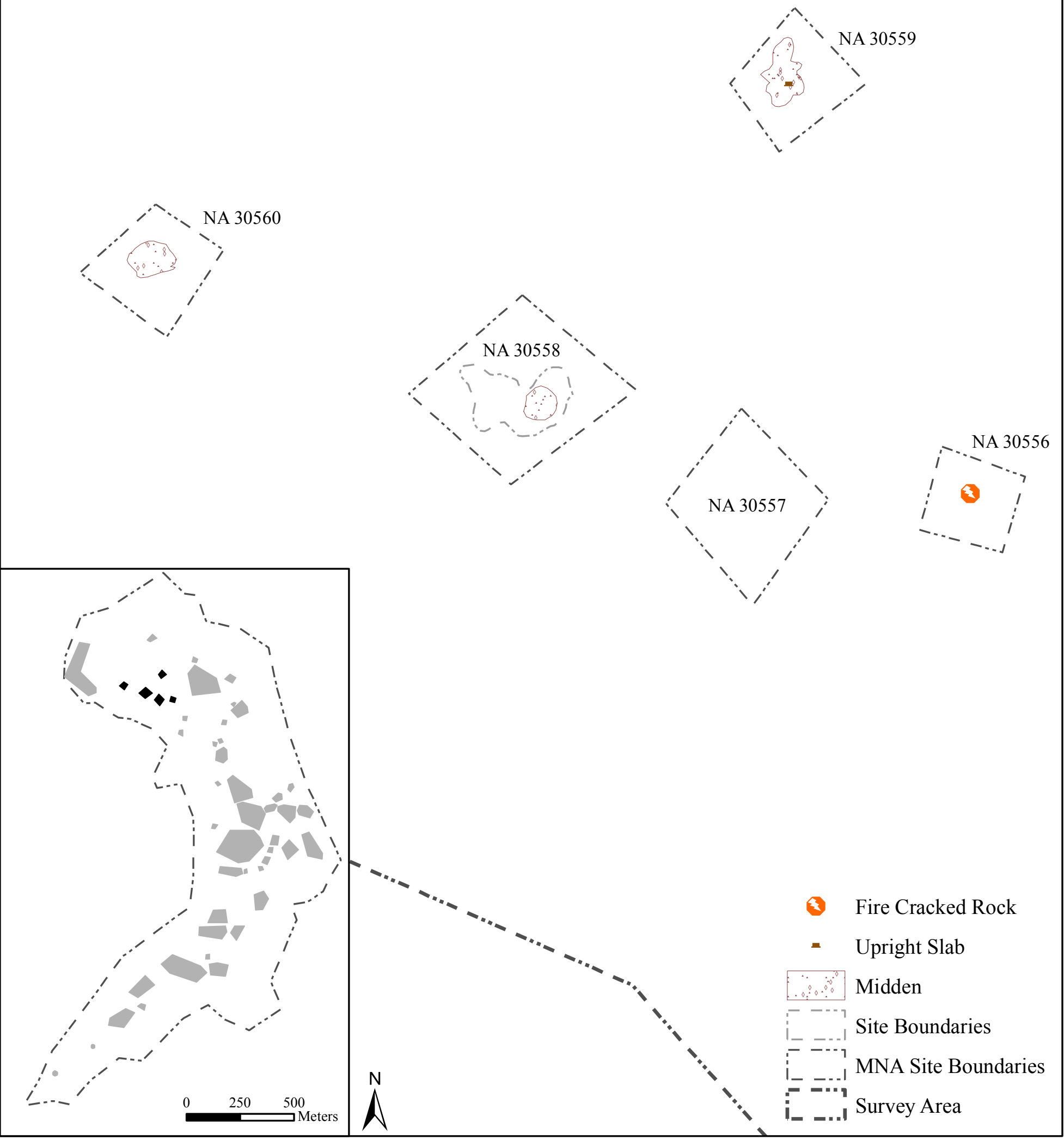




\section{Mungyaovi Survey}

NA30550 - NA30555; NA30561

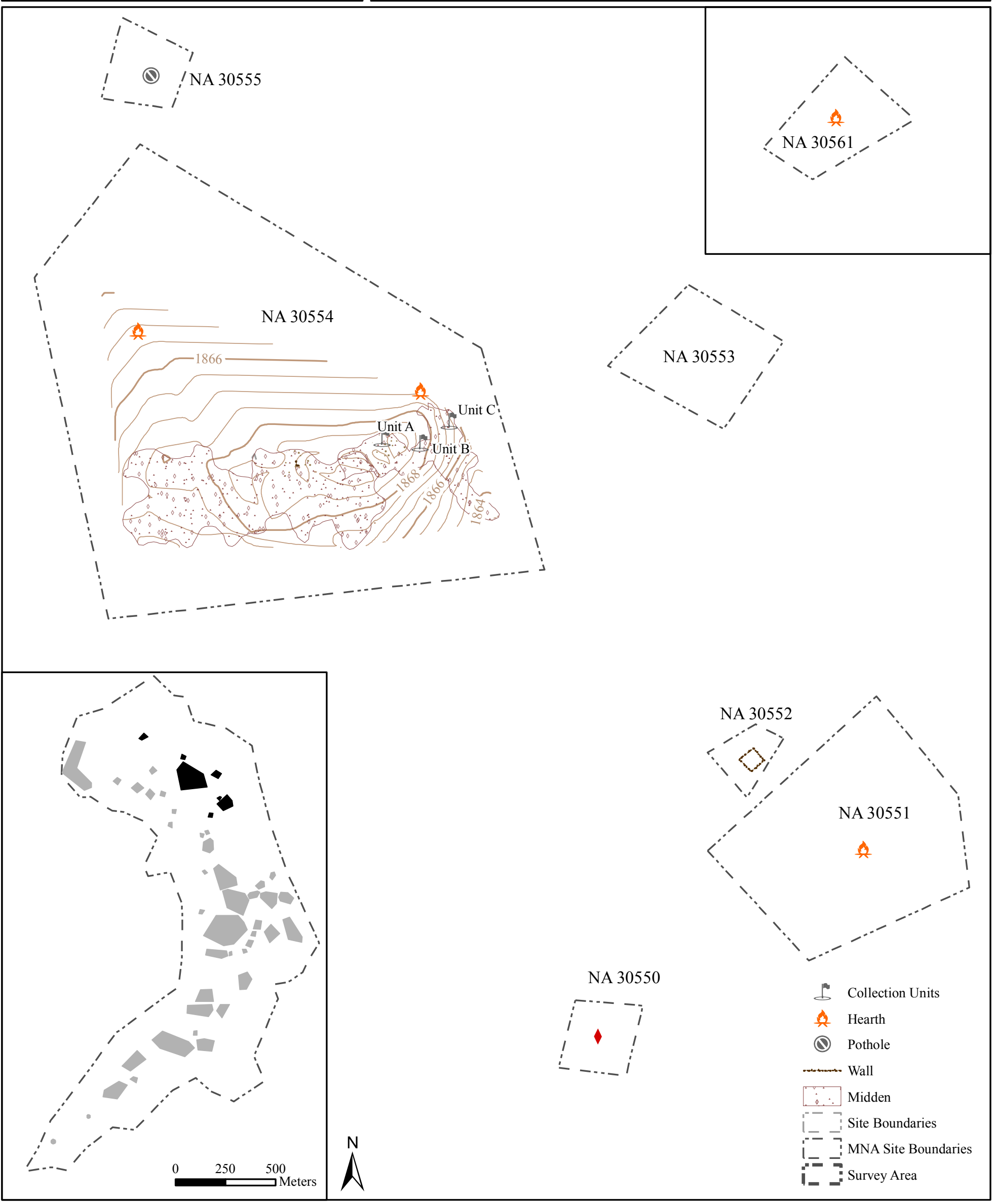




\section{Mungyaovi Survey}
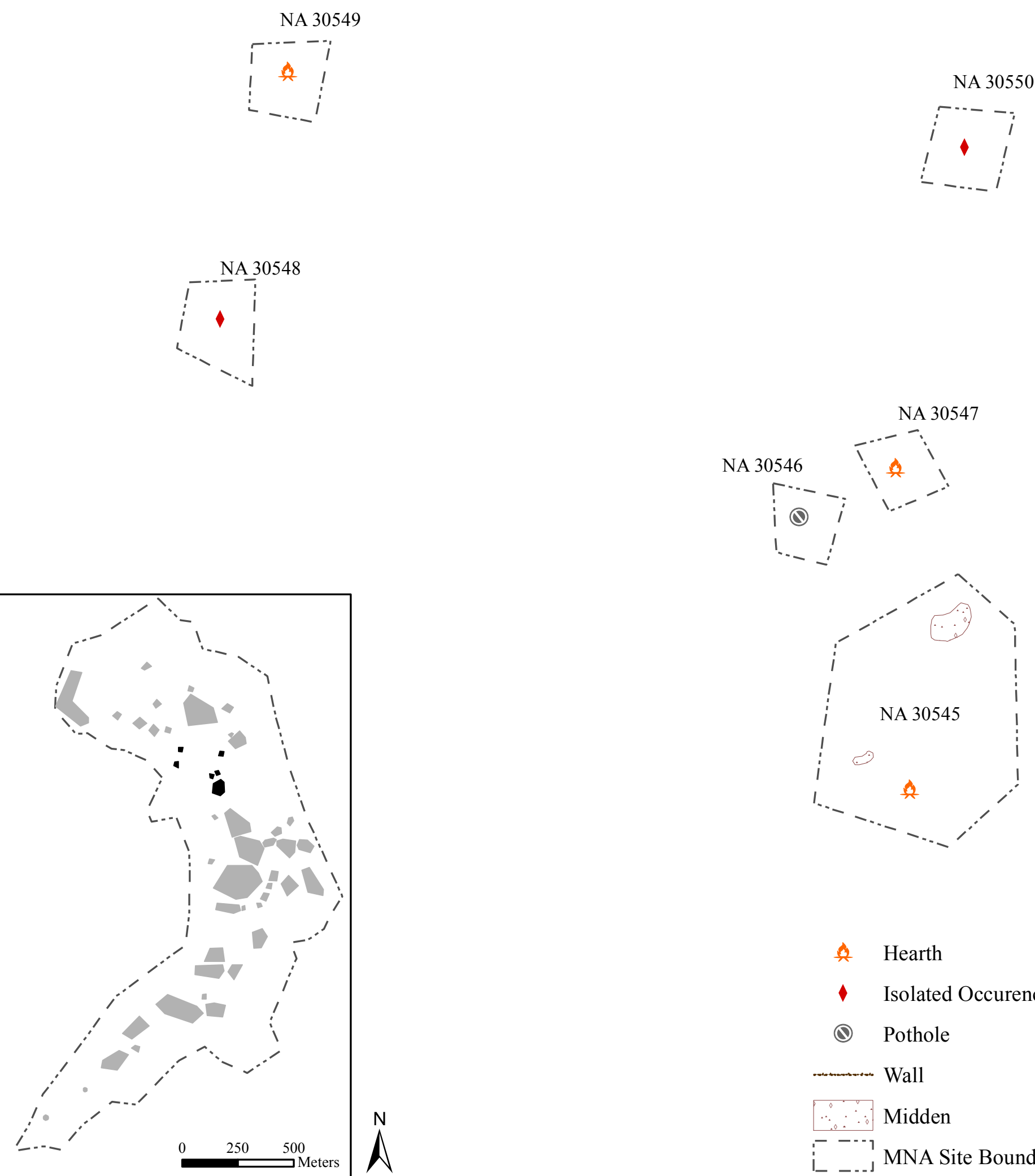

兵 Hearth

- Isolated Occurence

(a) Pothole

Wall

Midden

ב 


\section{Mungyaovi Survey}

\section{NA30543; NA30544; NA30537}

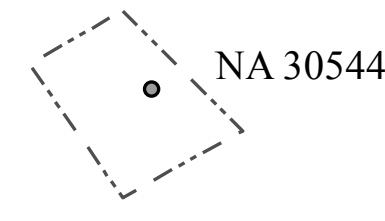

Cist

Collection Units

3 Fire Cracked Rock

兵 Hearth

- Clay Source

¿ Fire Craked Rock Polygon

$\because \div$ Midden

L
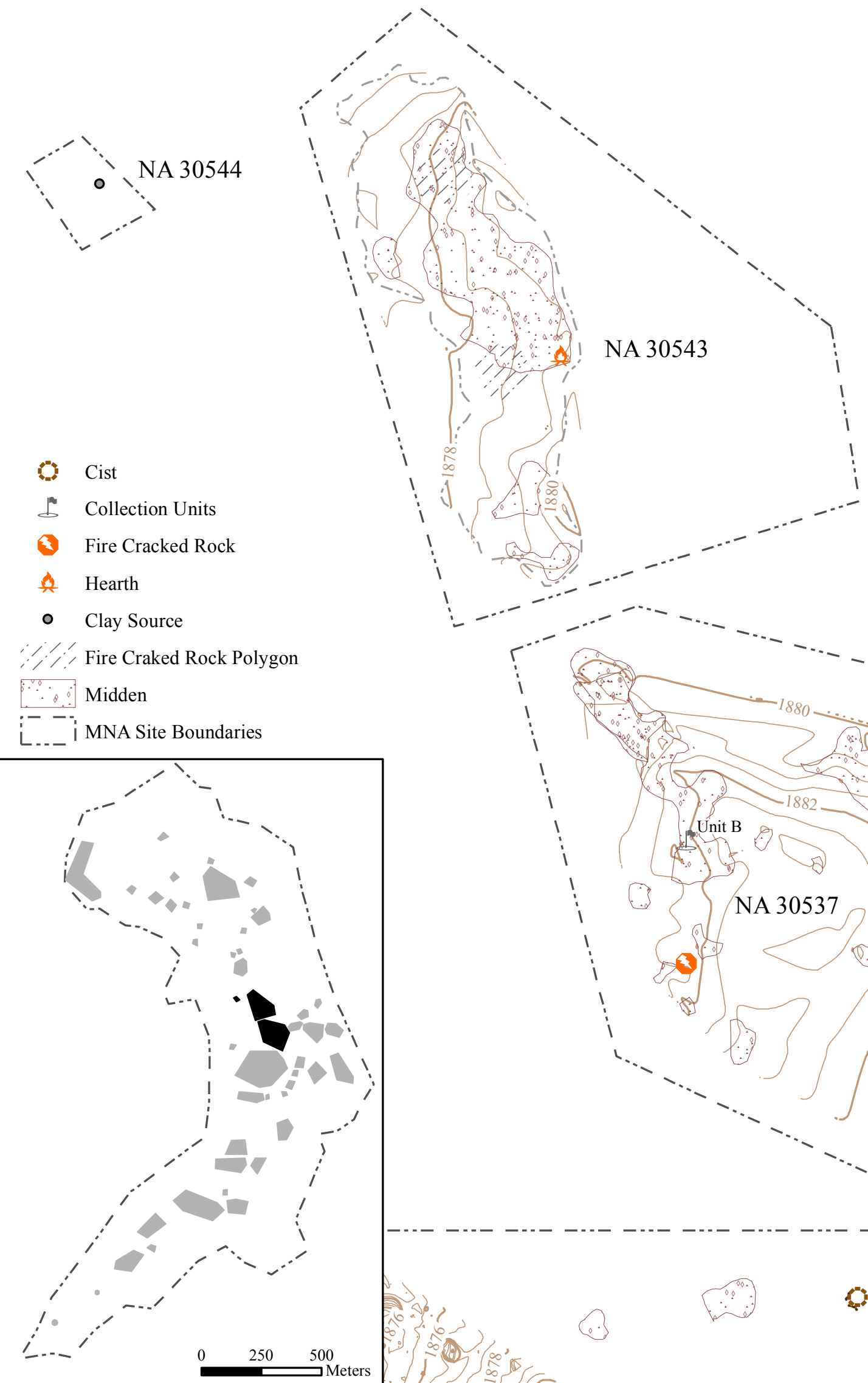


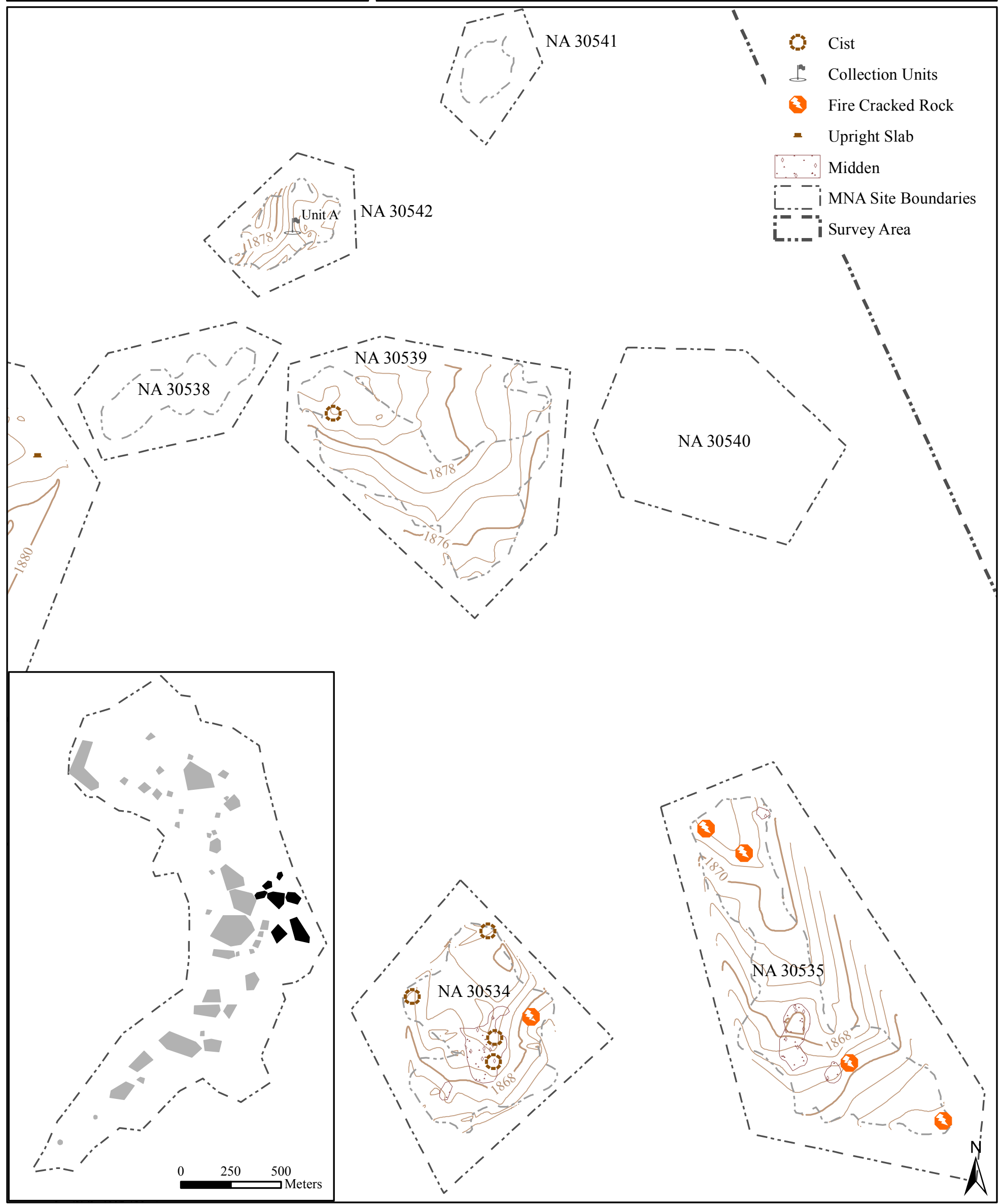




\section{Mungyaovi Survey}

\section{NA30526 - NA30533}

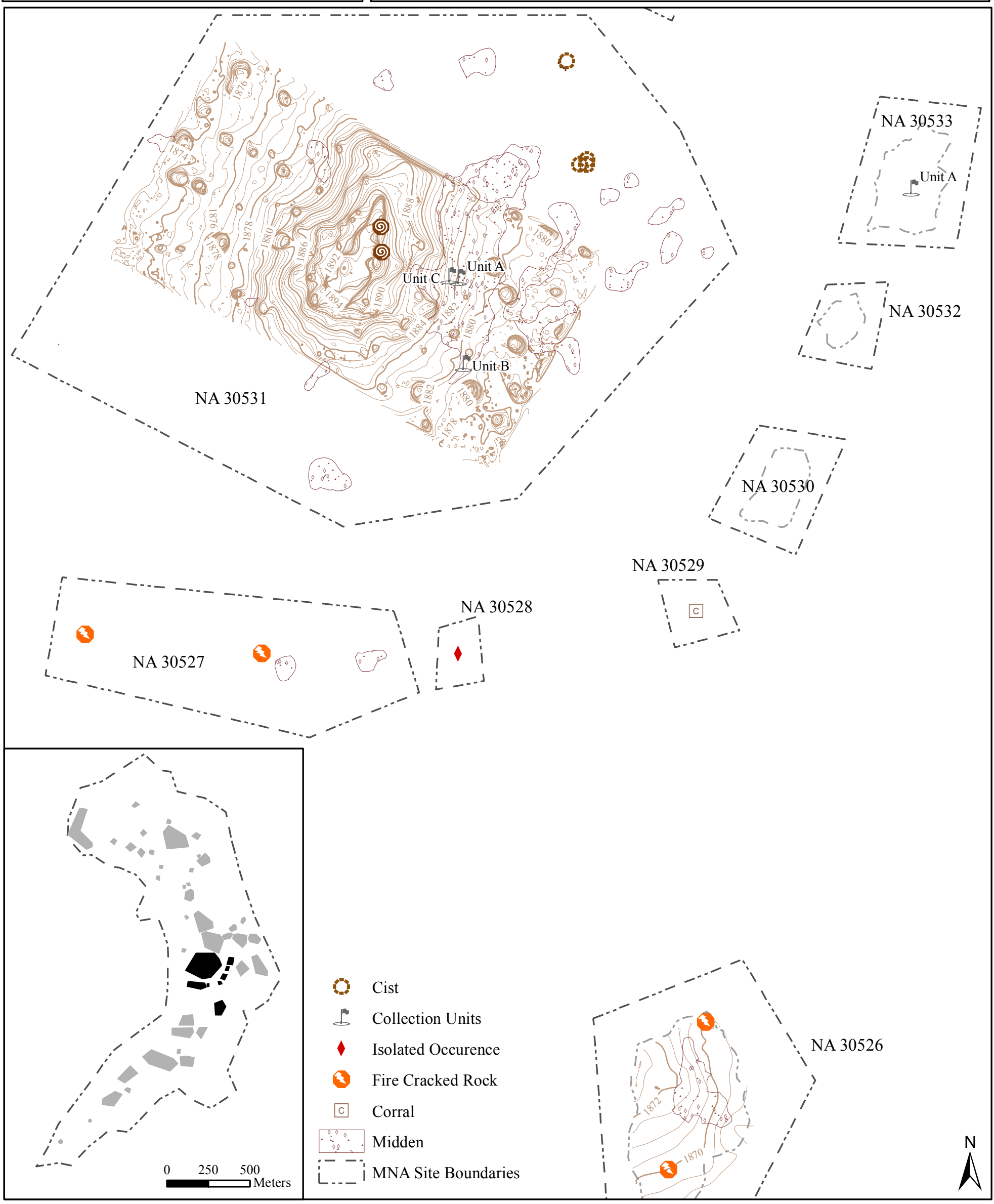




\section{Mungyaovi Survey}

\section{NA30520 - NA30525}

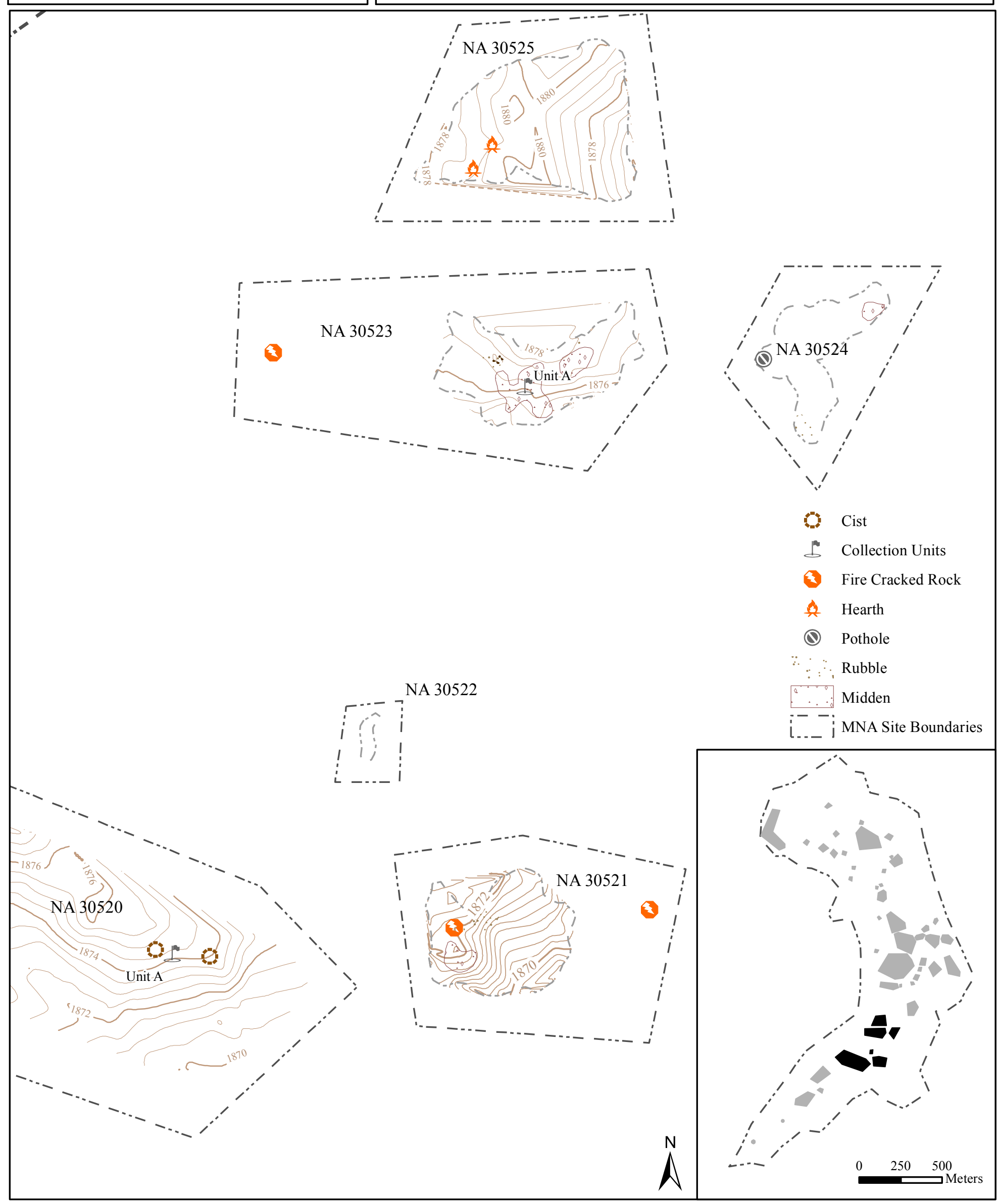




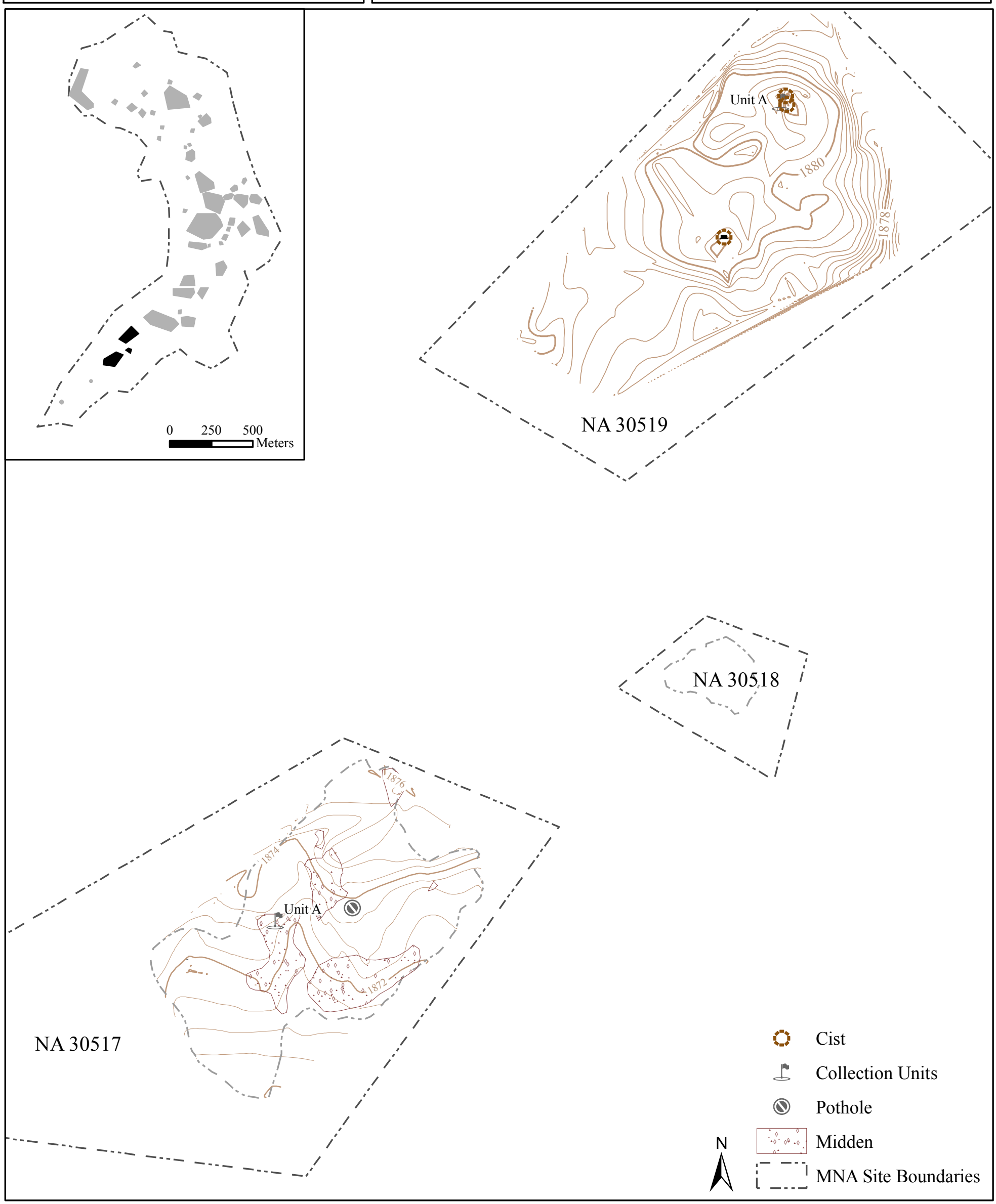




\section{Nakya'ovi}

NA28333

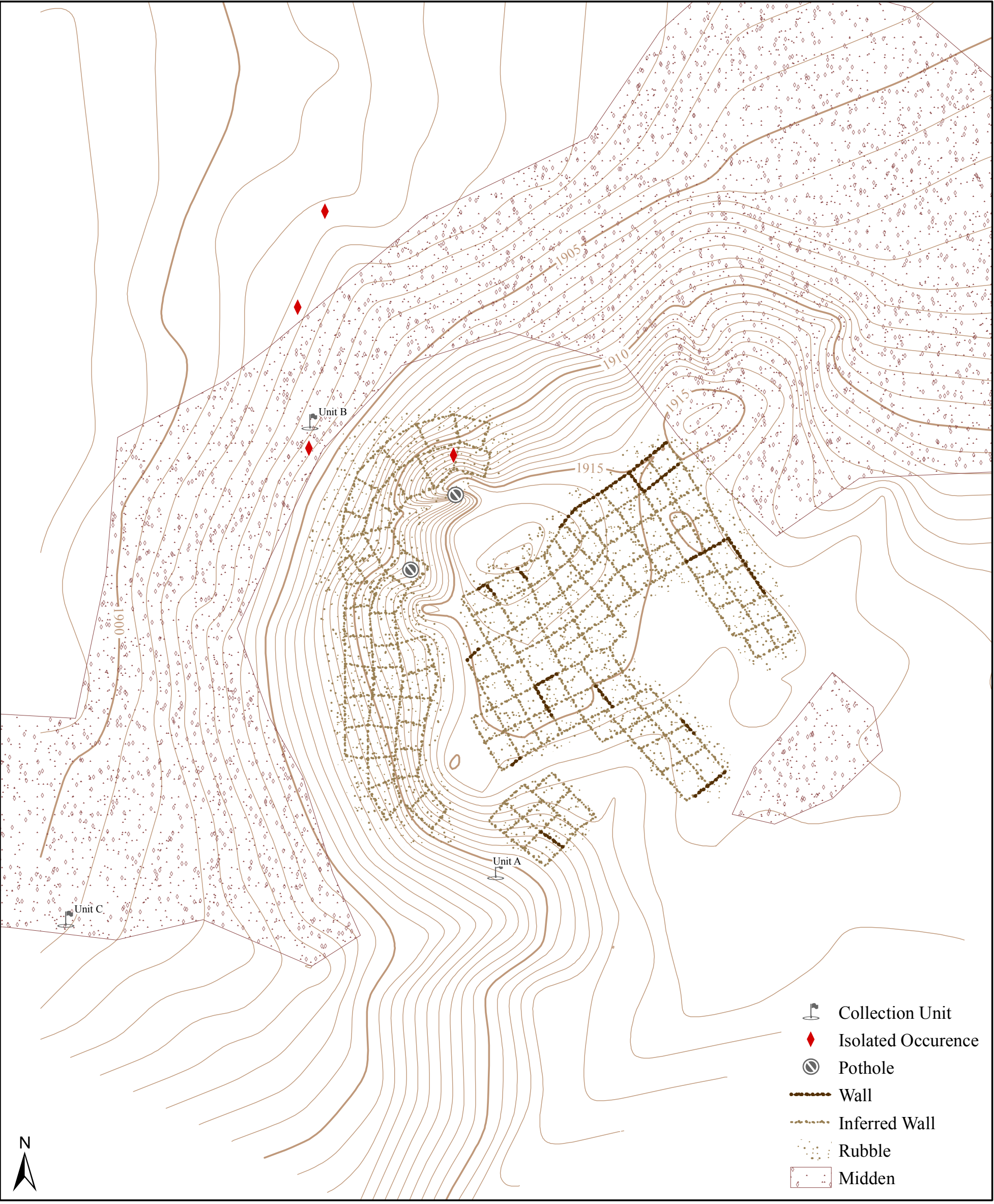




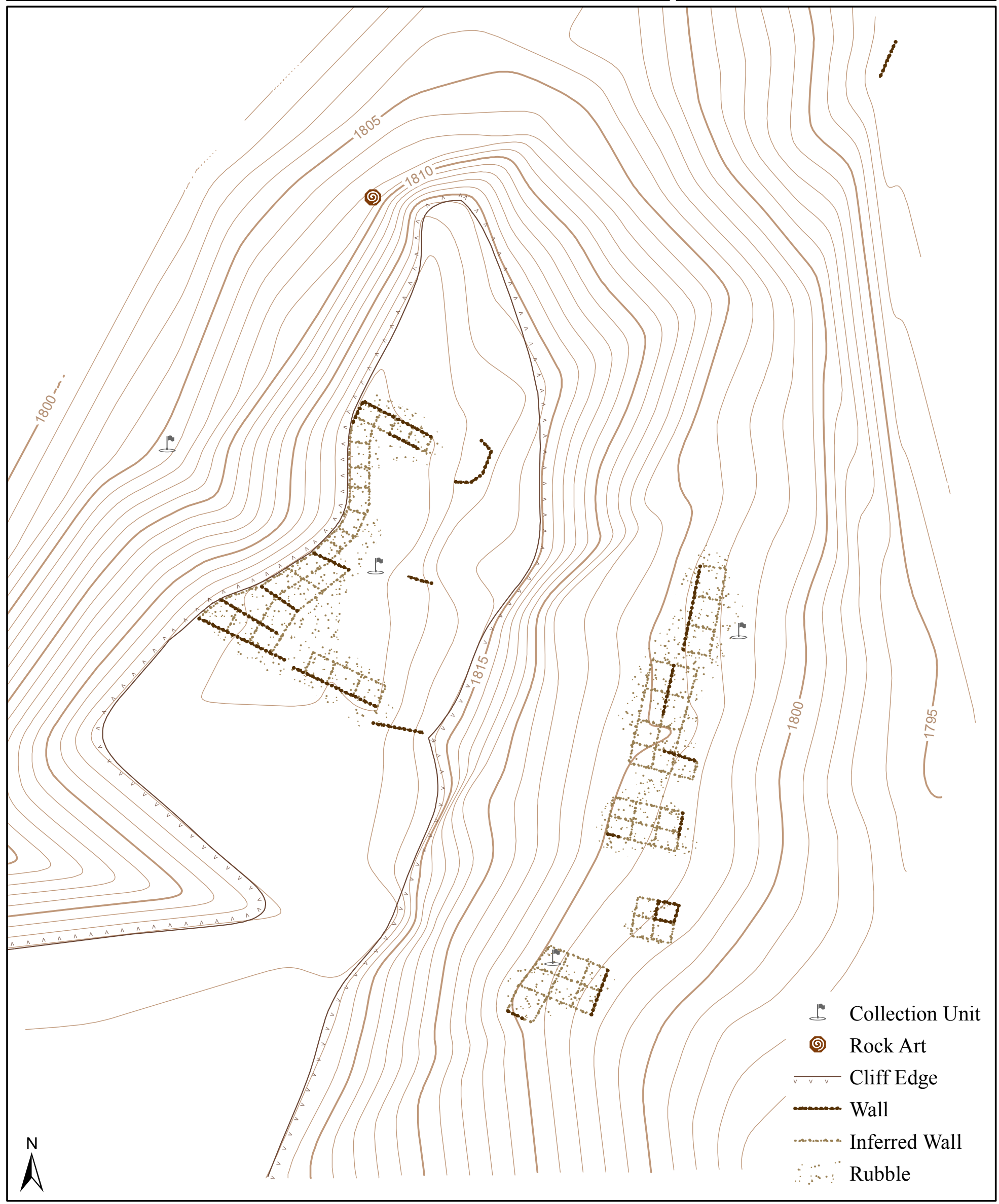




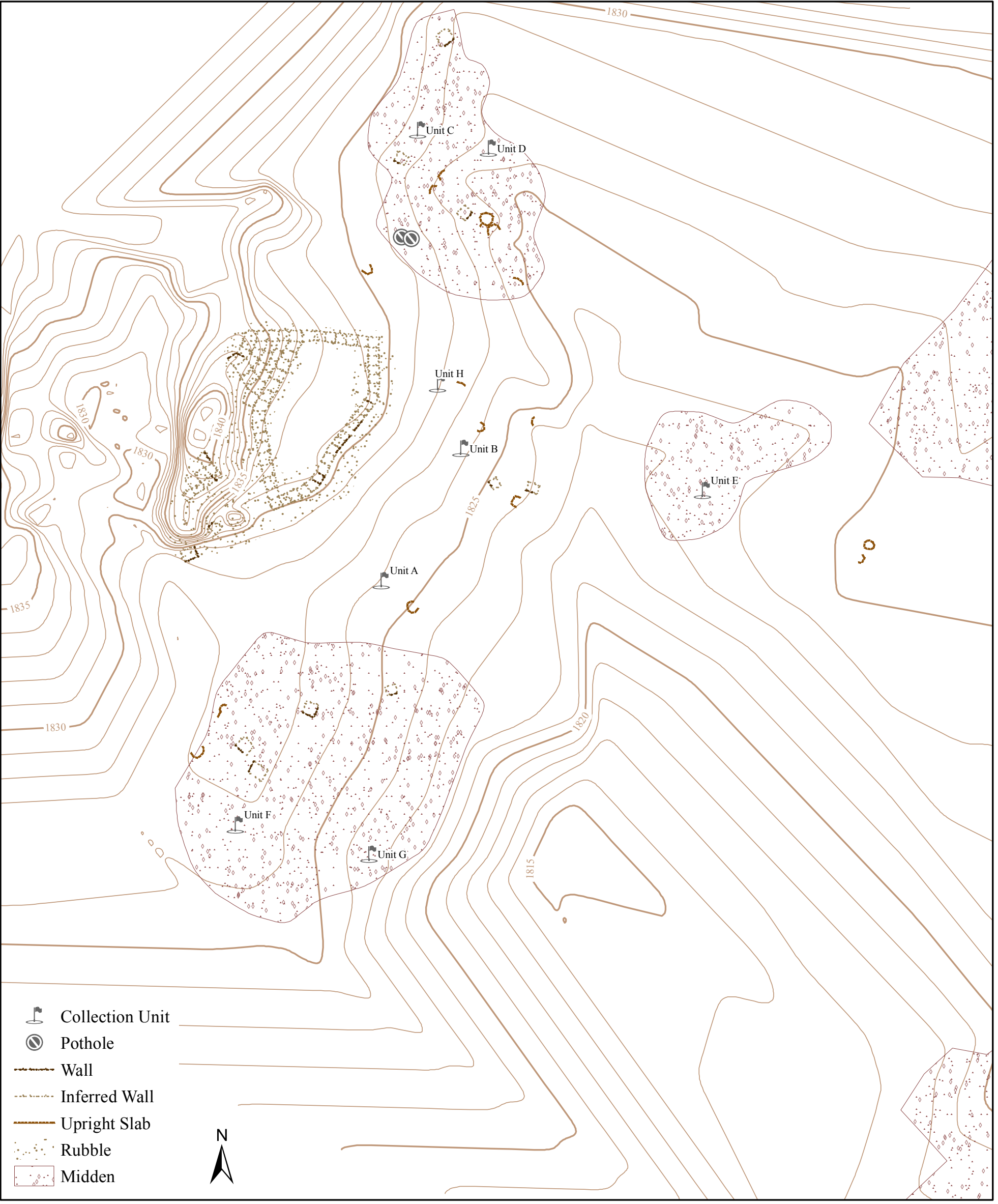




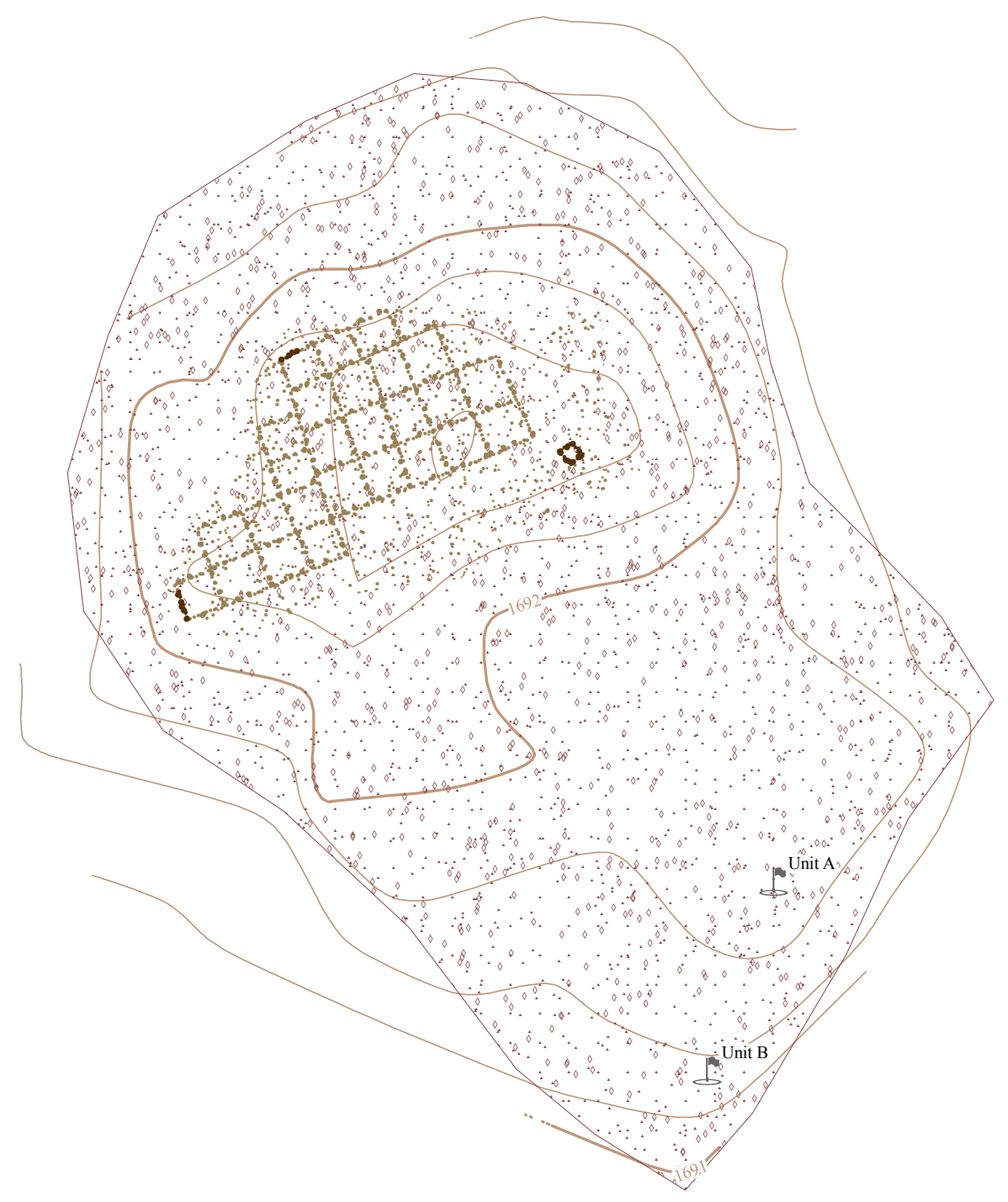

Collection Unit

-monomos. Wall

.......... Inferred Wall 


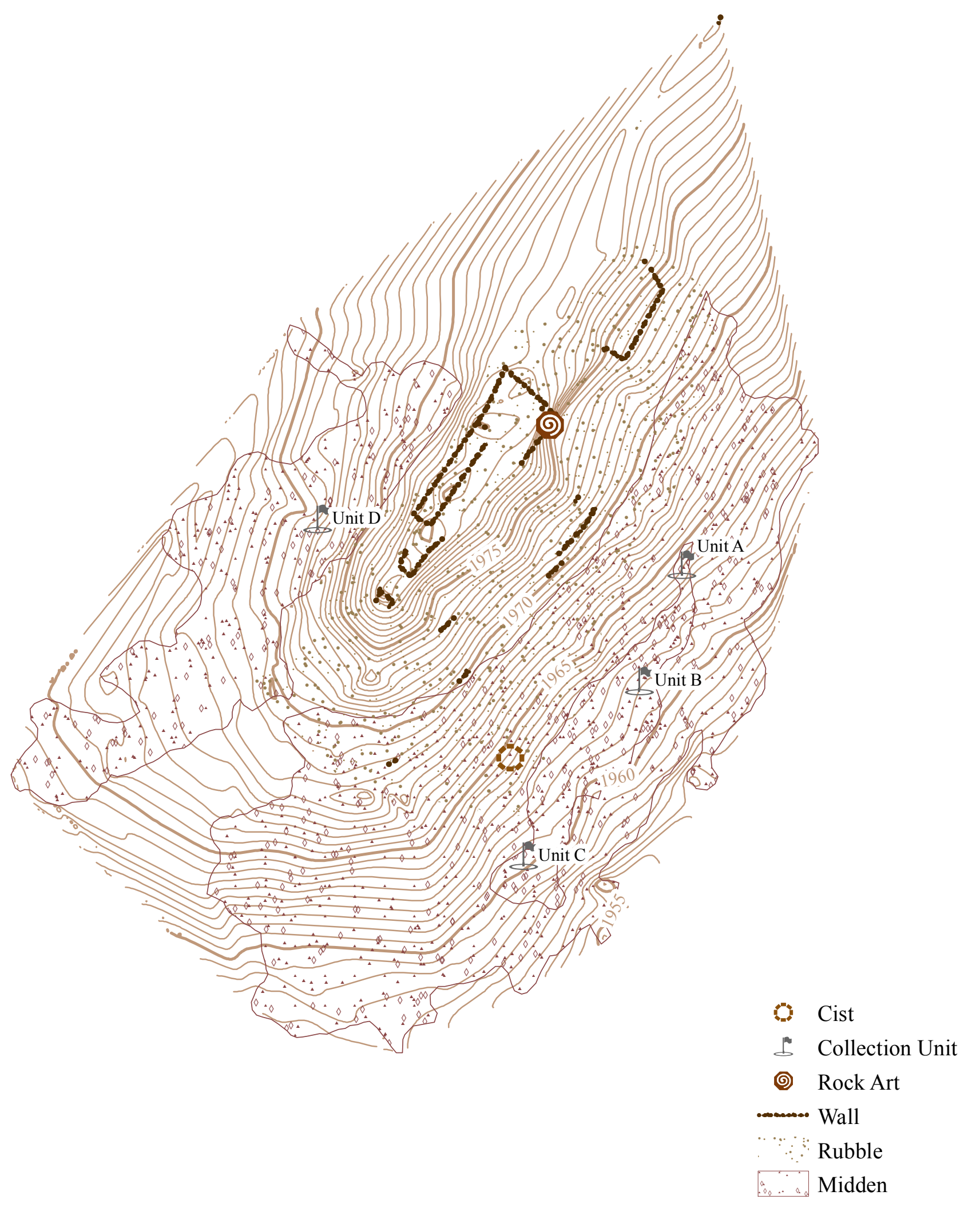




\section{Payupki}

NA1040






\section{\begin{tabular}{|l|l}
\hline Pink Arrow & NA1001
\end{tabular}}

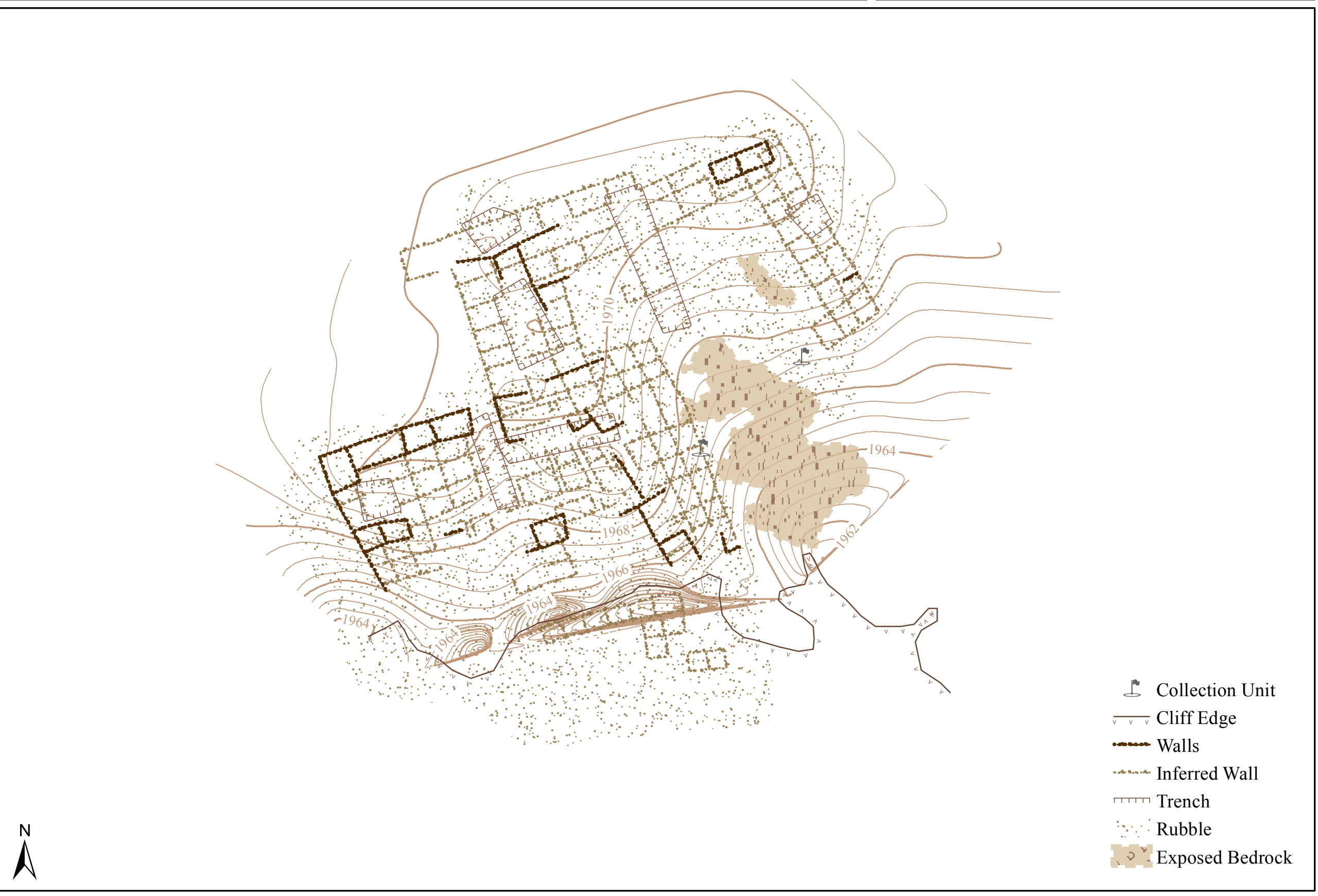




\section{Pivanhonkiopi}

NA1169

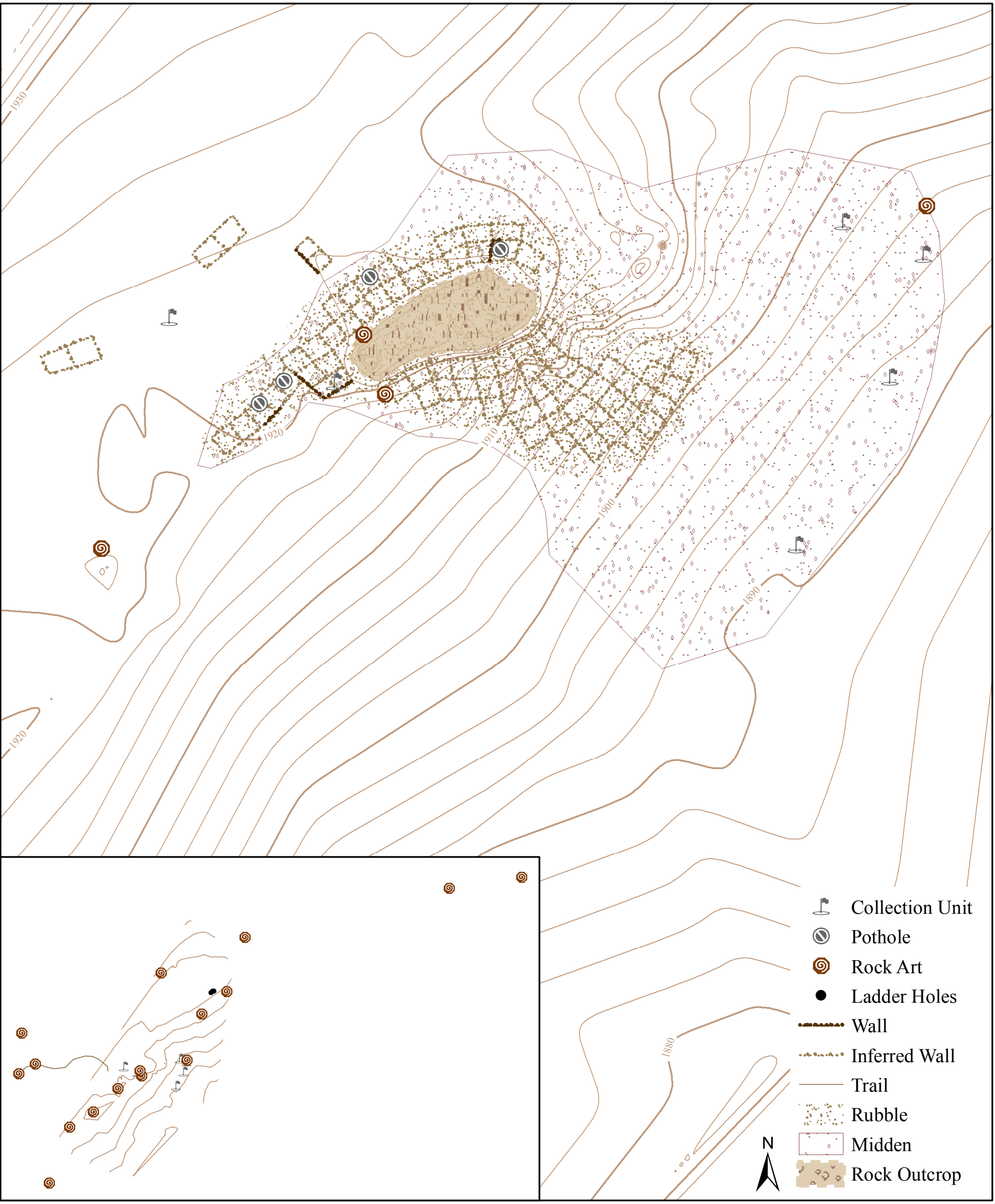




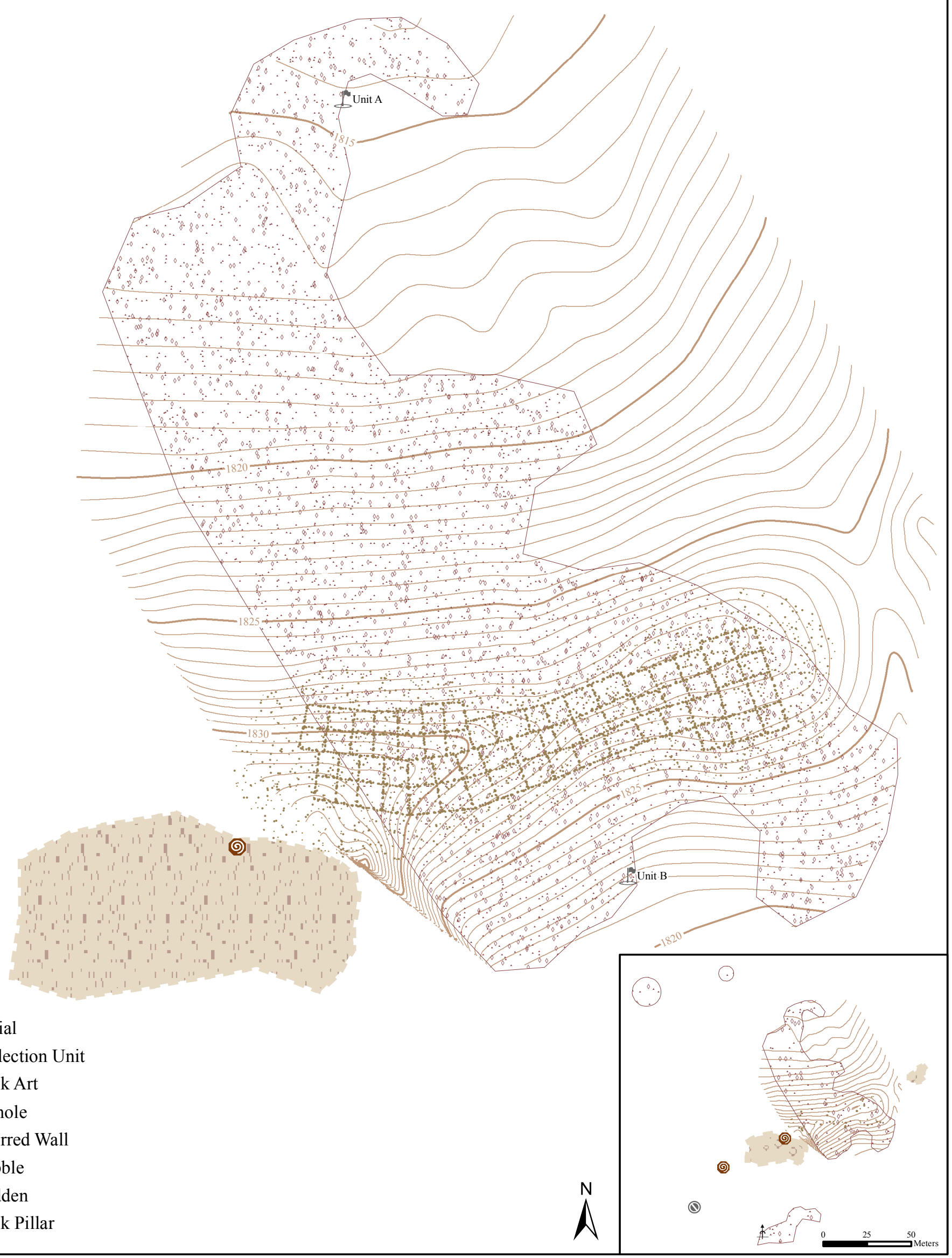

止 Burial

(b) Collection Unit

(2) Rock Art

(a) Pothole

......... Inferred Wall

Rubble

Midden

Rock Pillar 


\section{Qaotaqtipu}

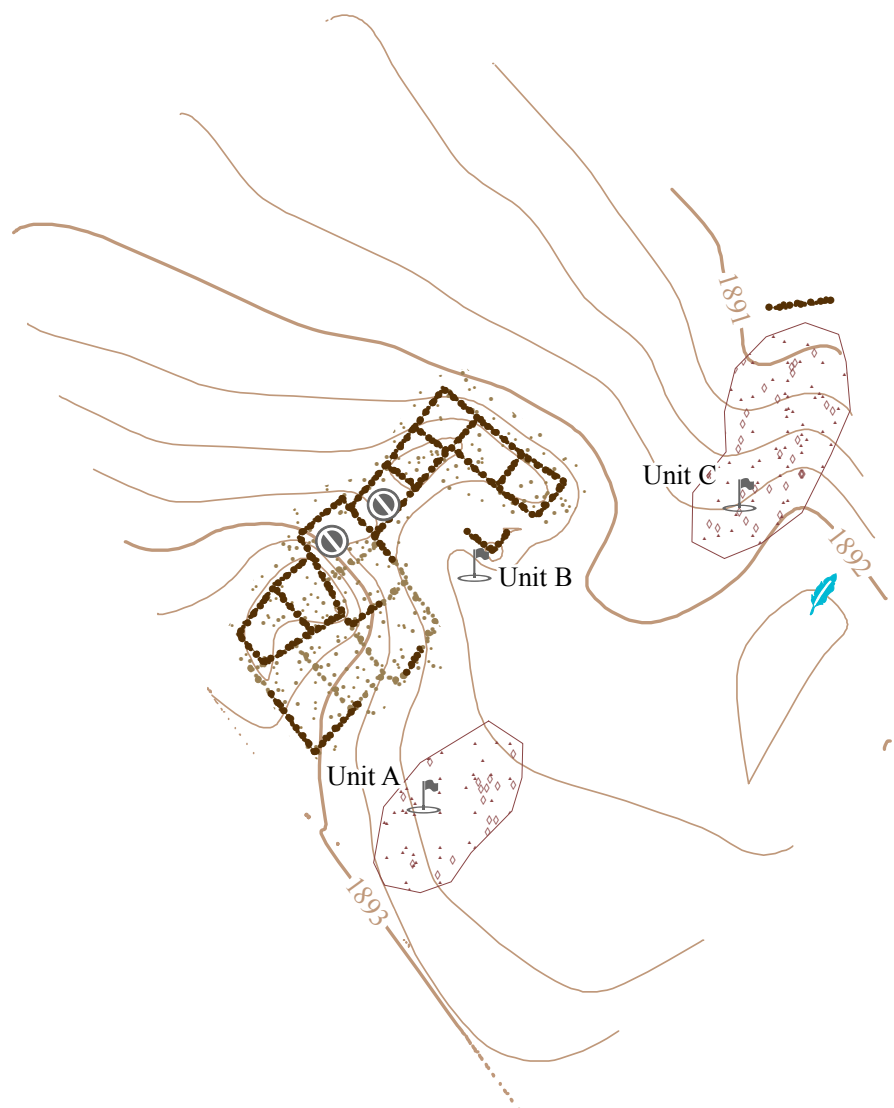

$\$$ Collection Unit

(1) Pothole

$\&$ Shrine

Wall

........ Inferred Wall

. . . Midden 


\section{Qootsaptuvela}

NA1699

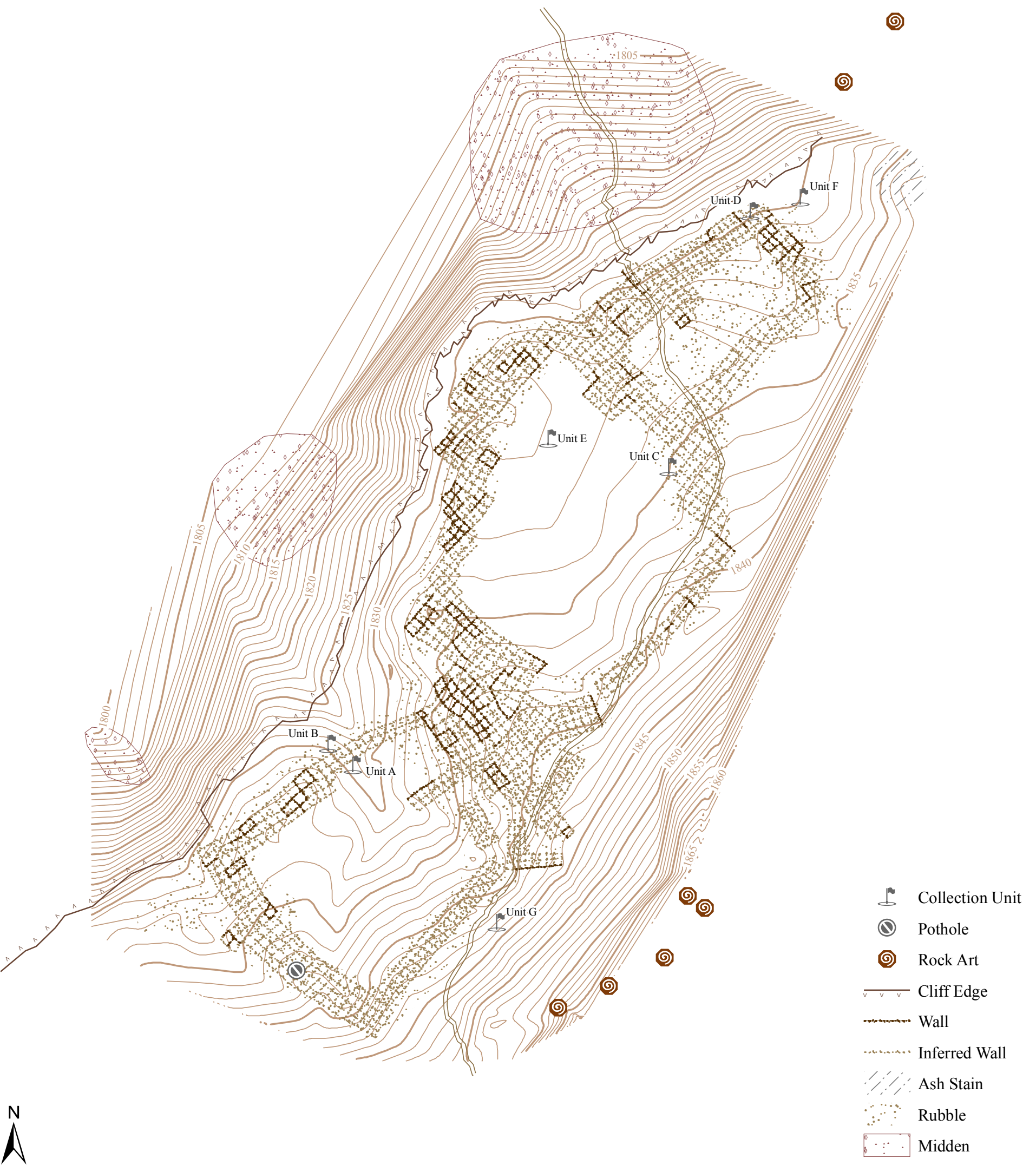




\section{Sanovi}

NA28332
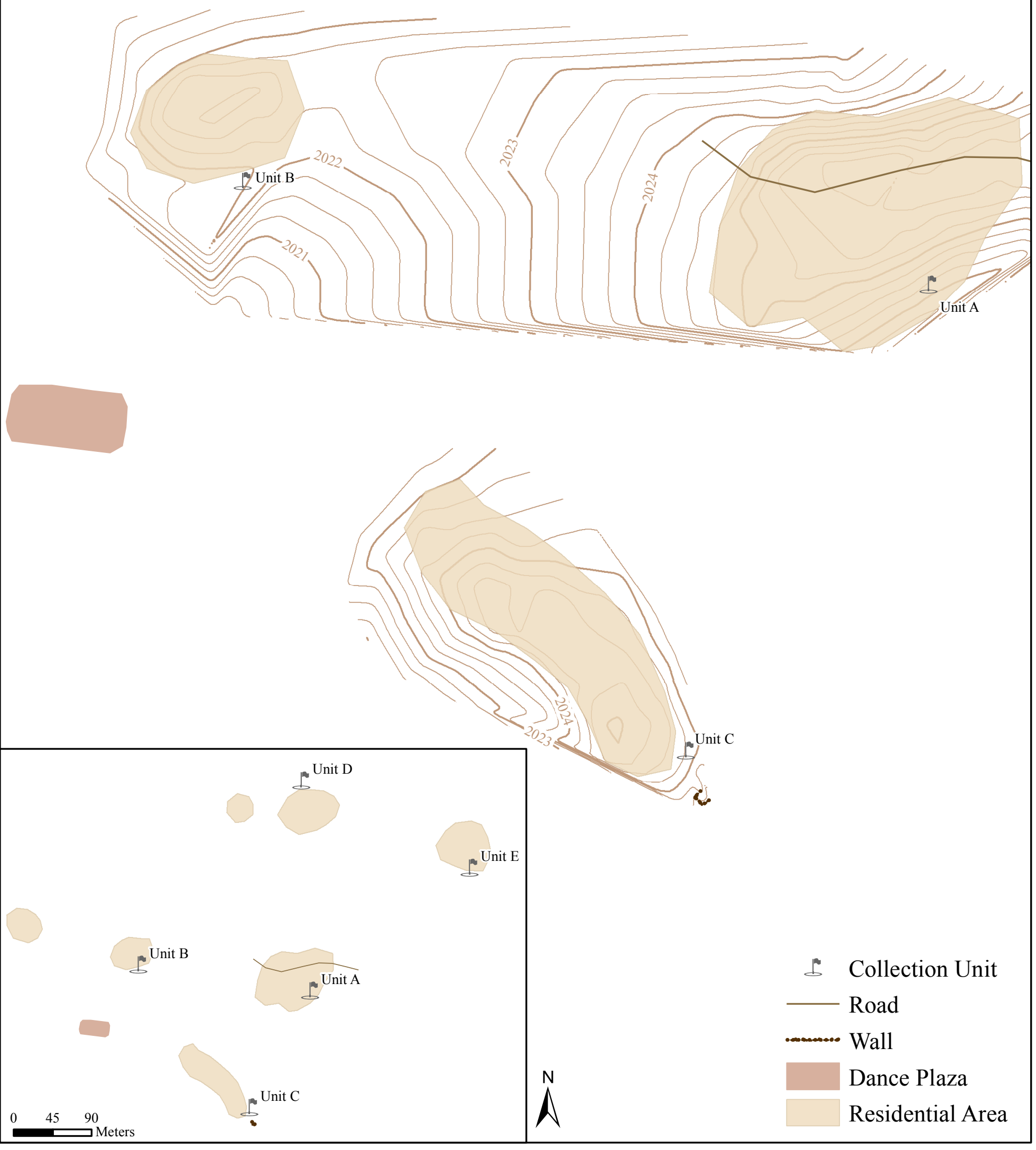


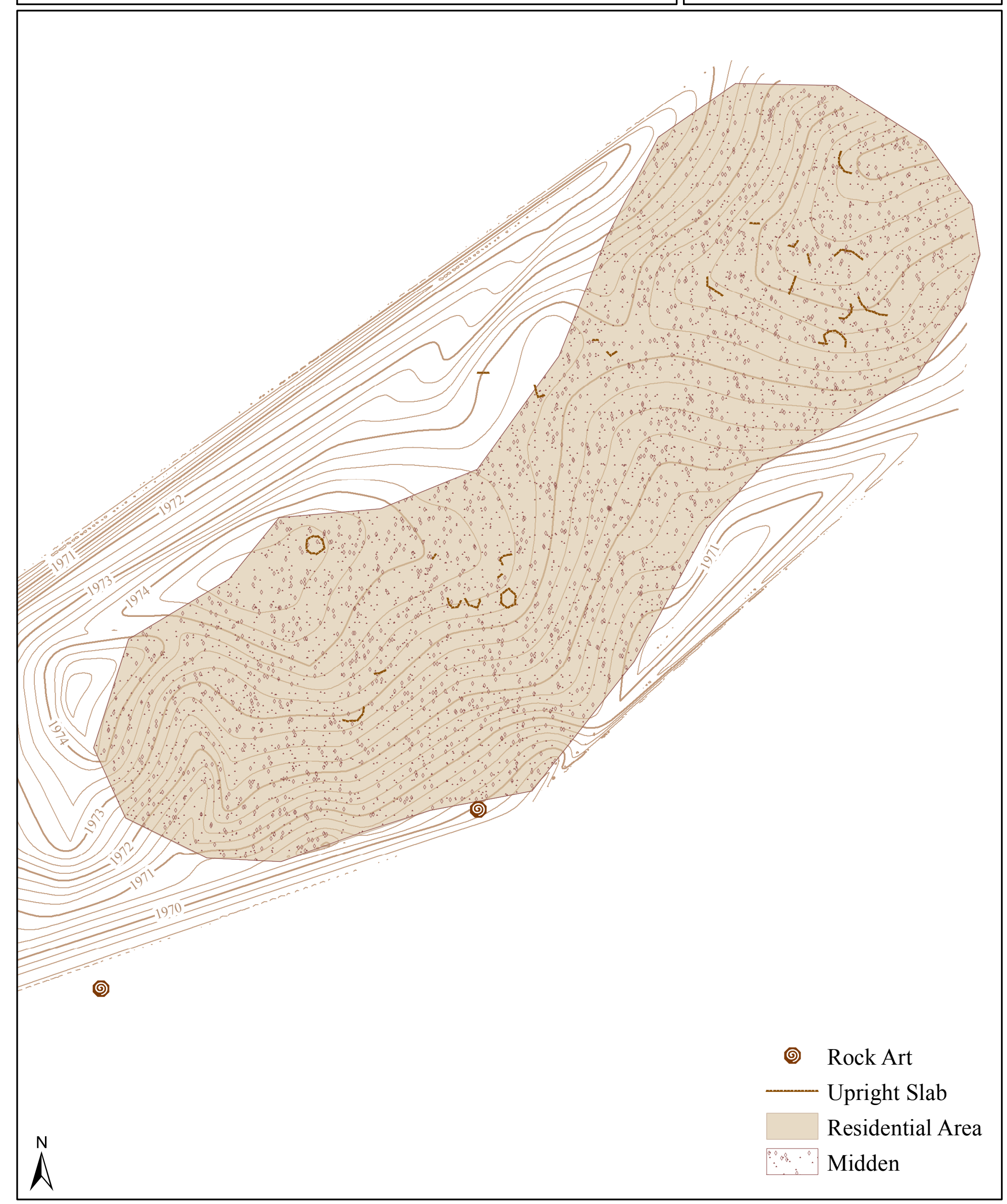




\section{West Sanovi}

NA28332

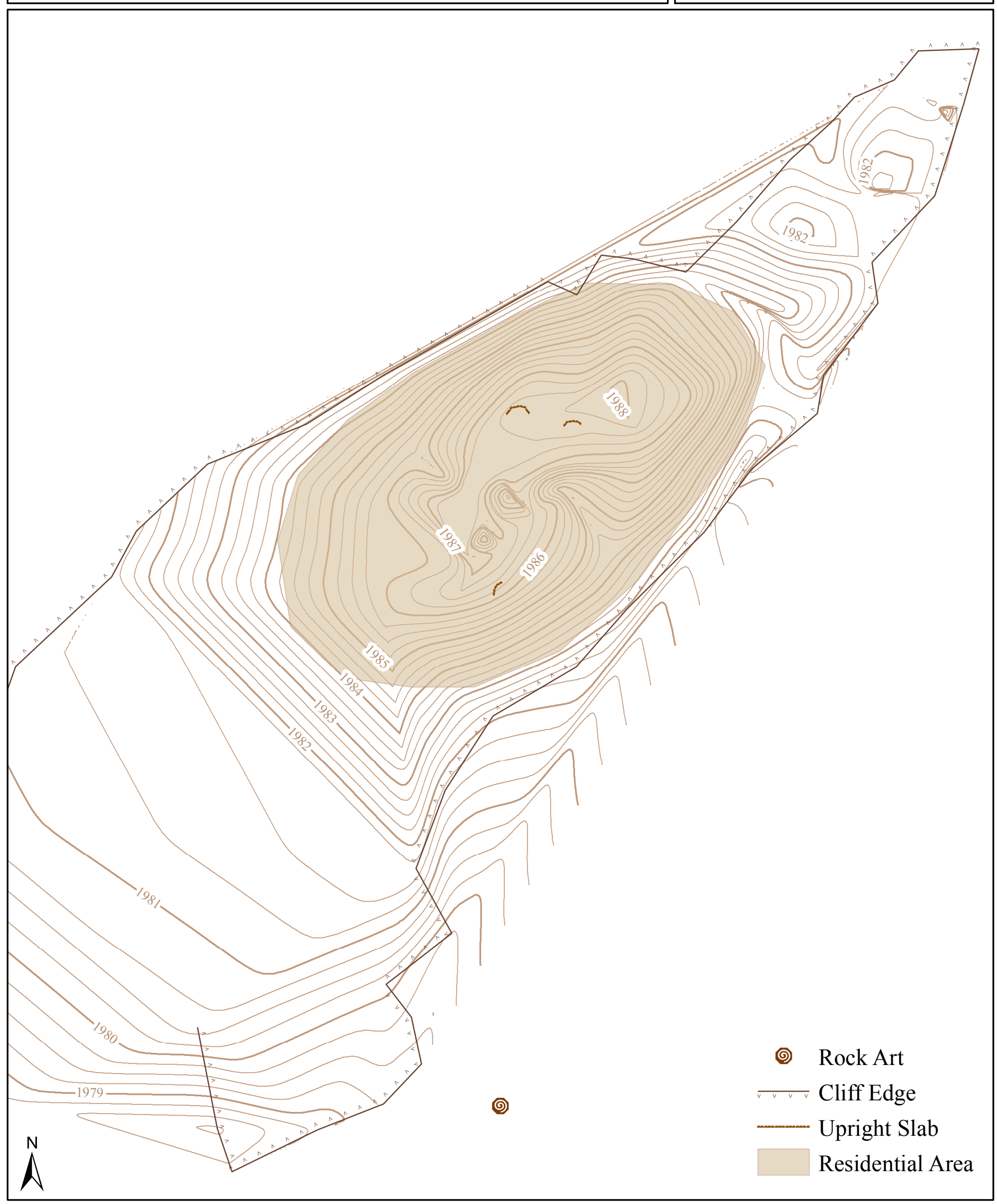




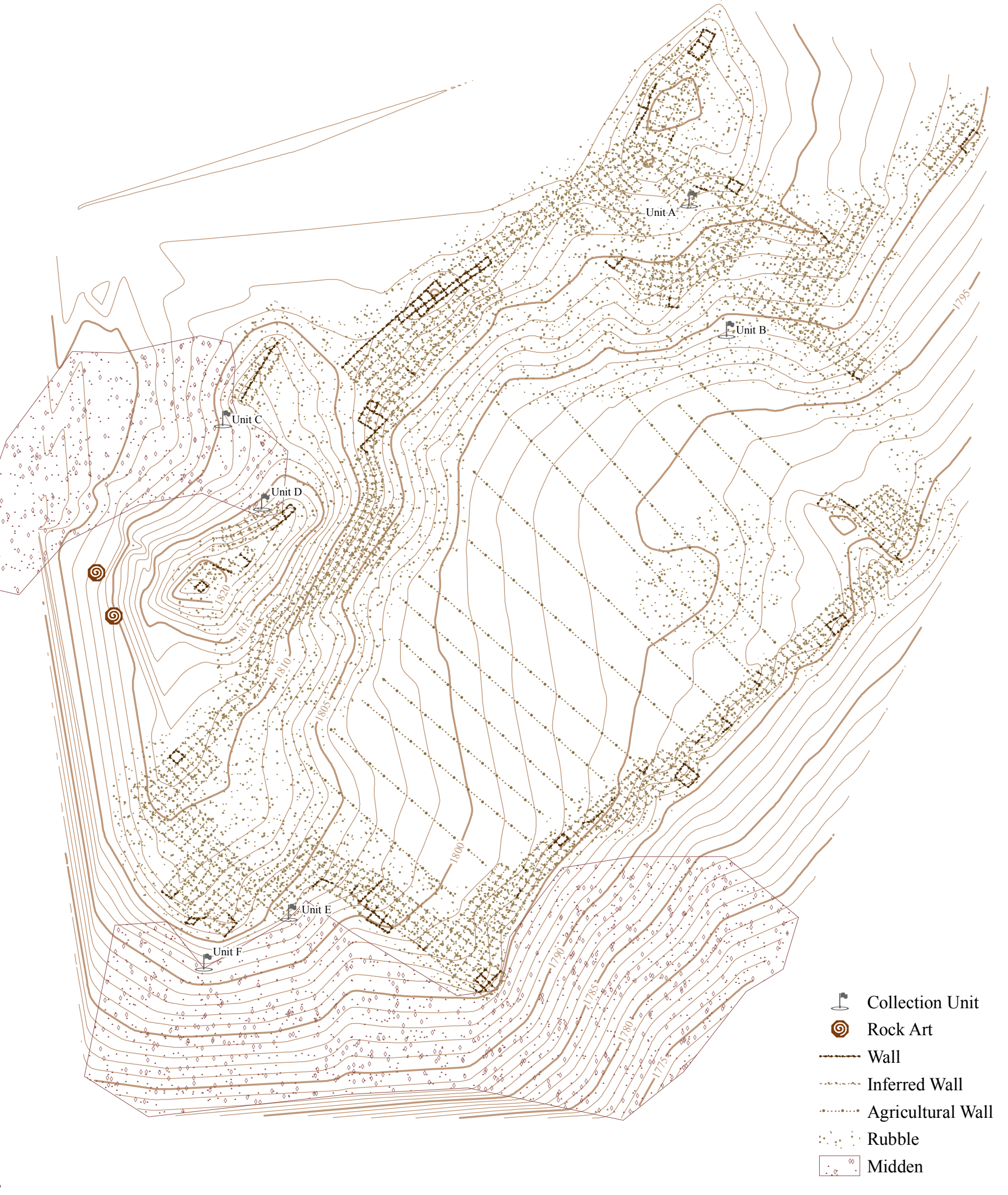




\section{Old Soongopavi

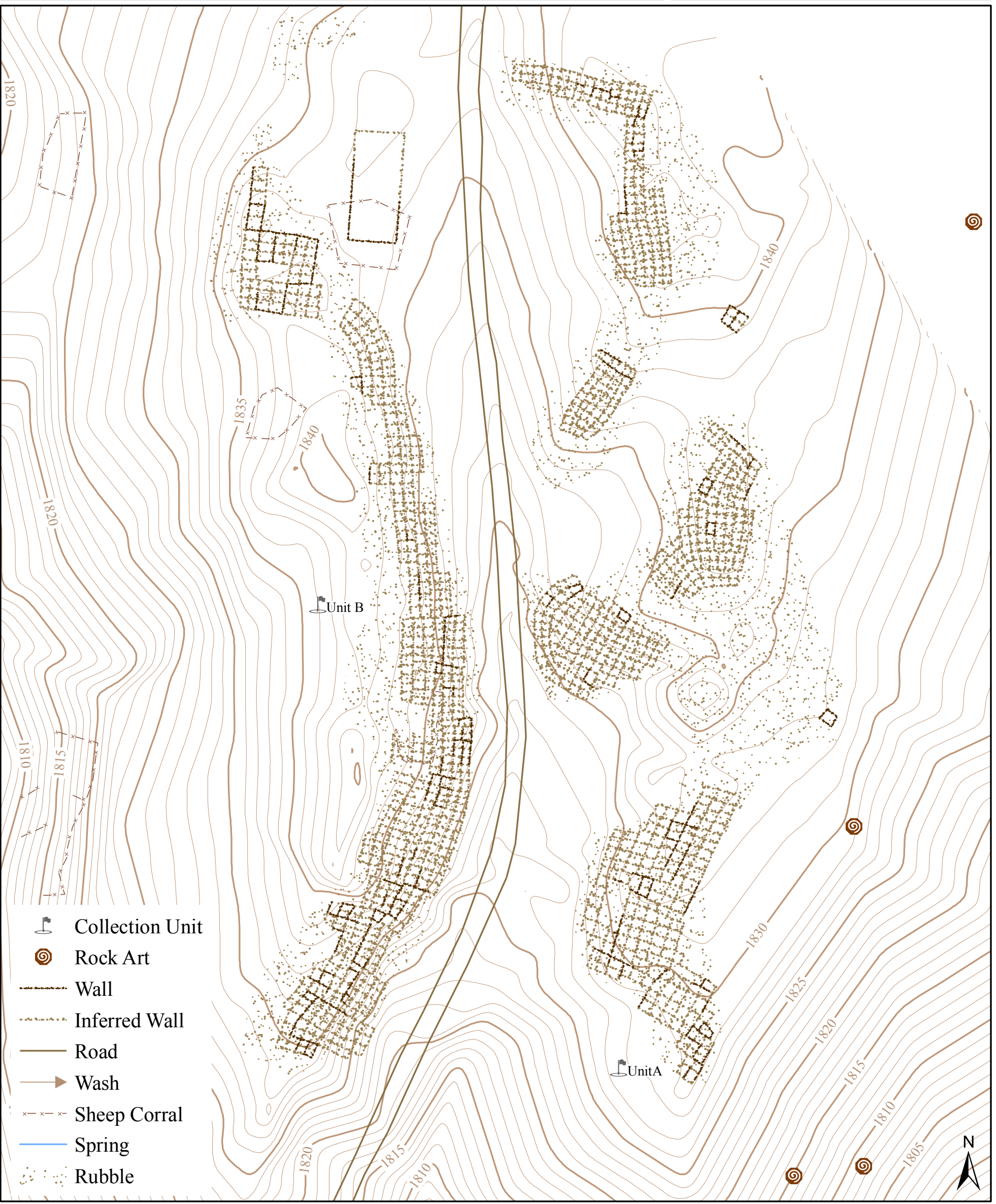




\section{Old Soongopavi \\ NA868}

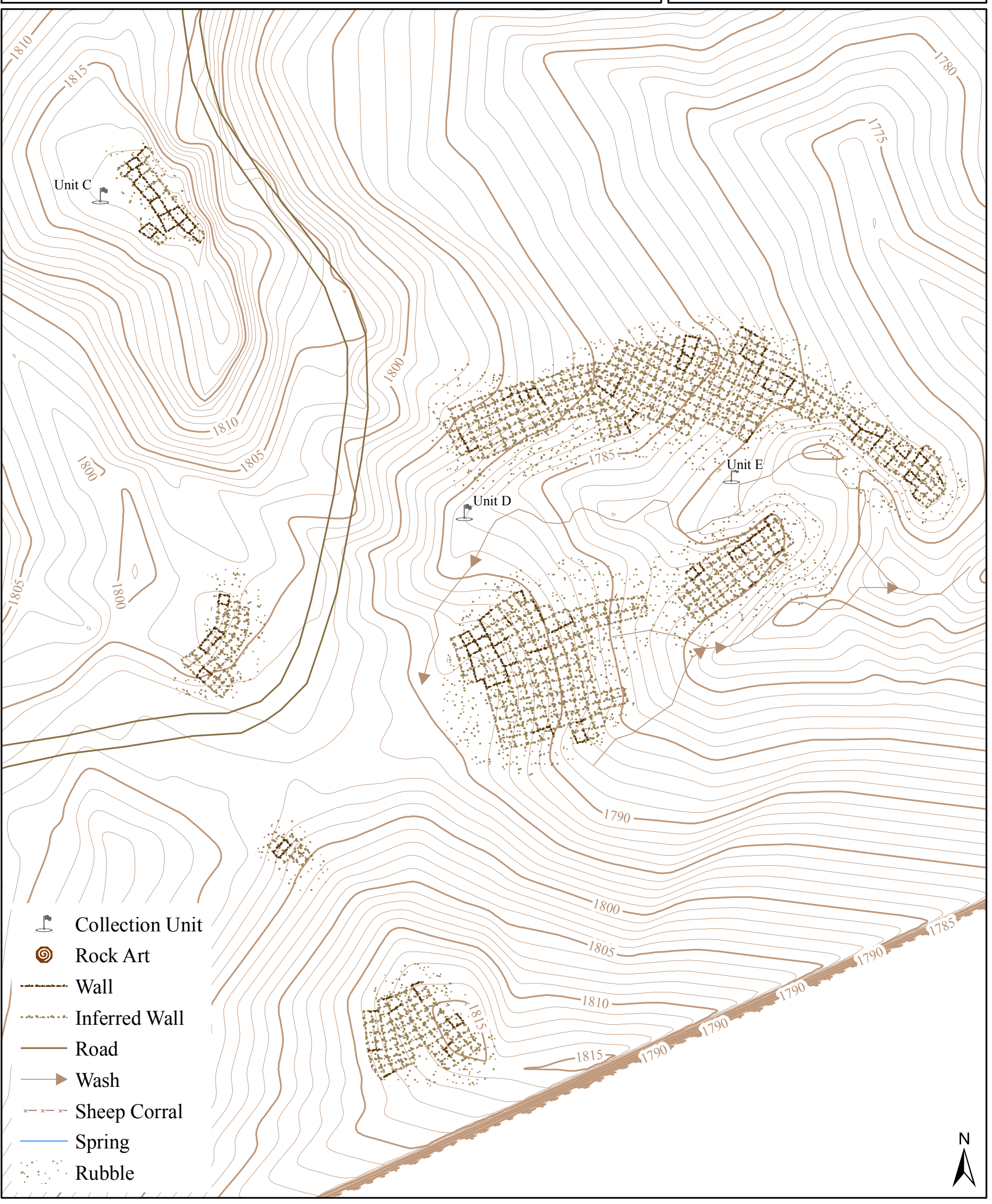




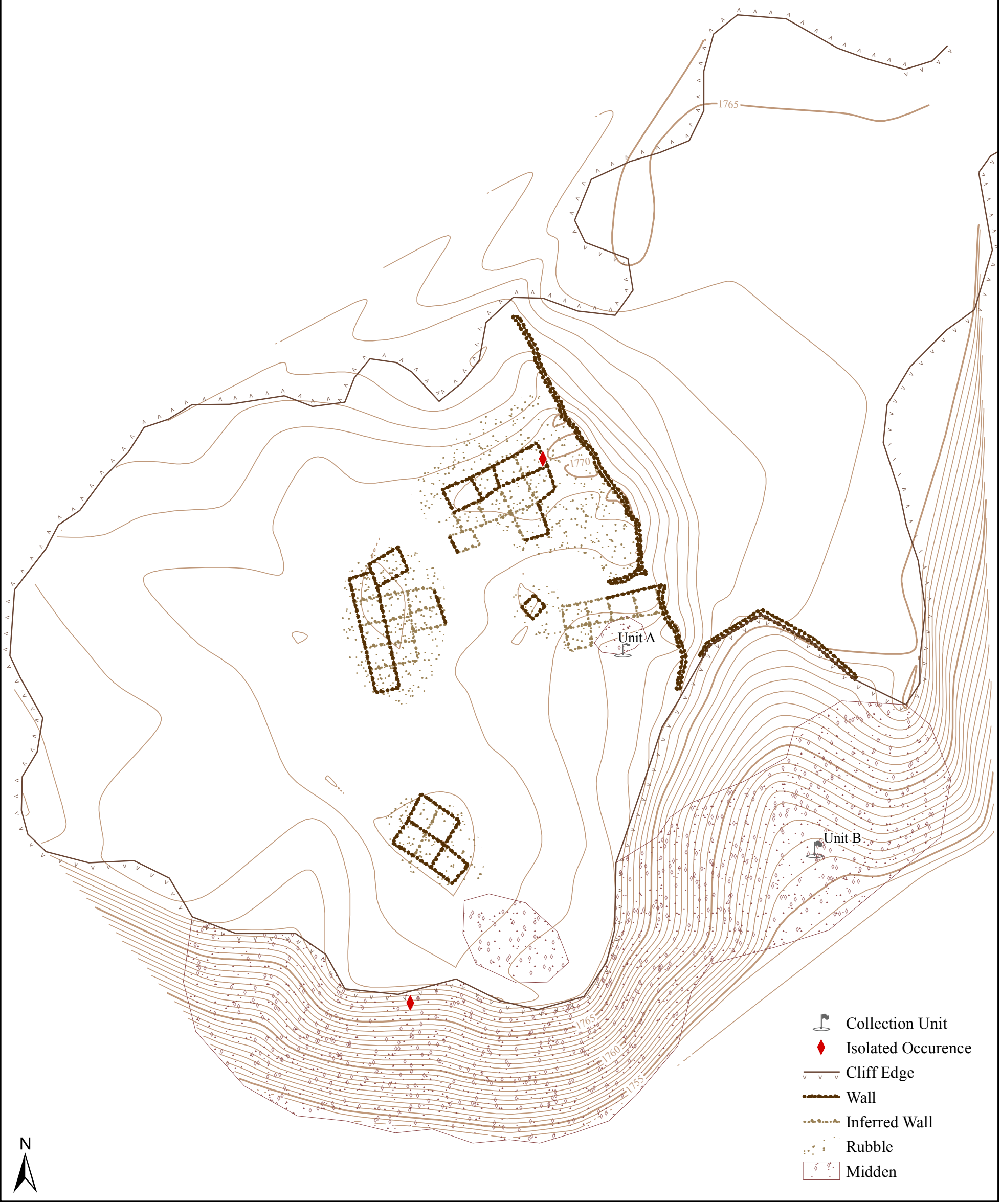




\section{Sun Forehead}

NA9502

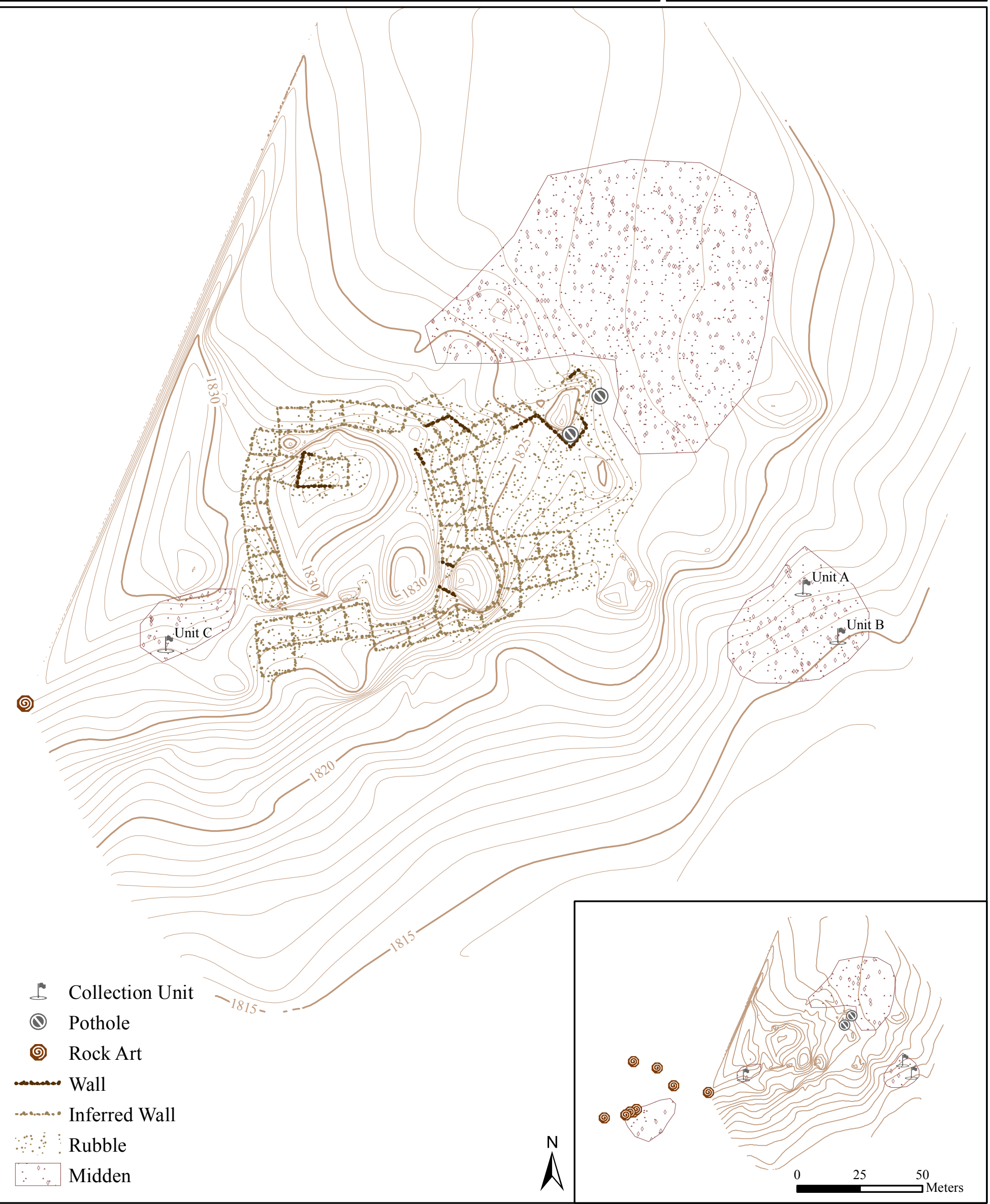




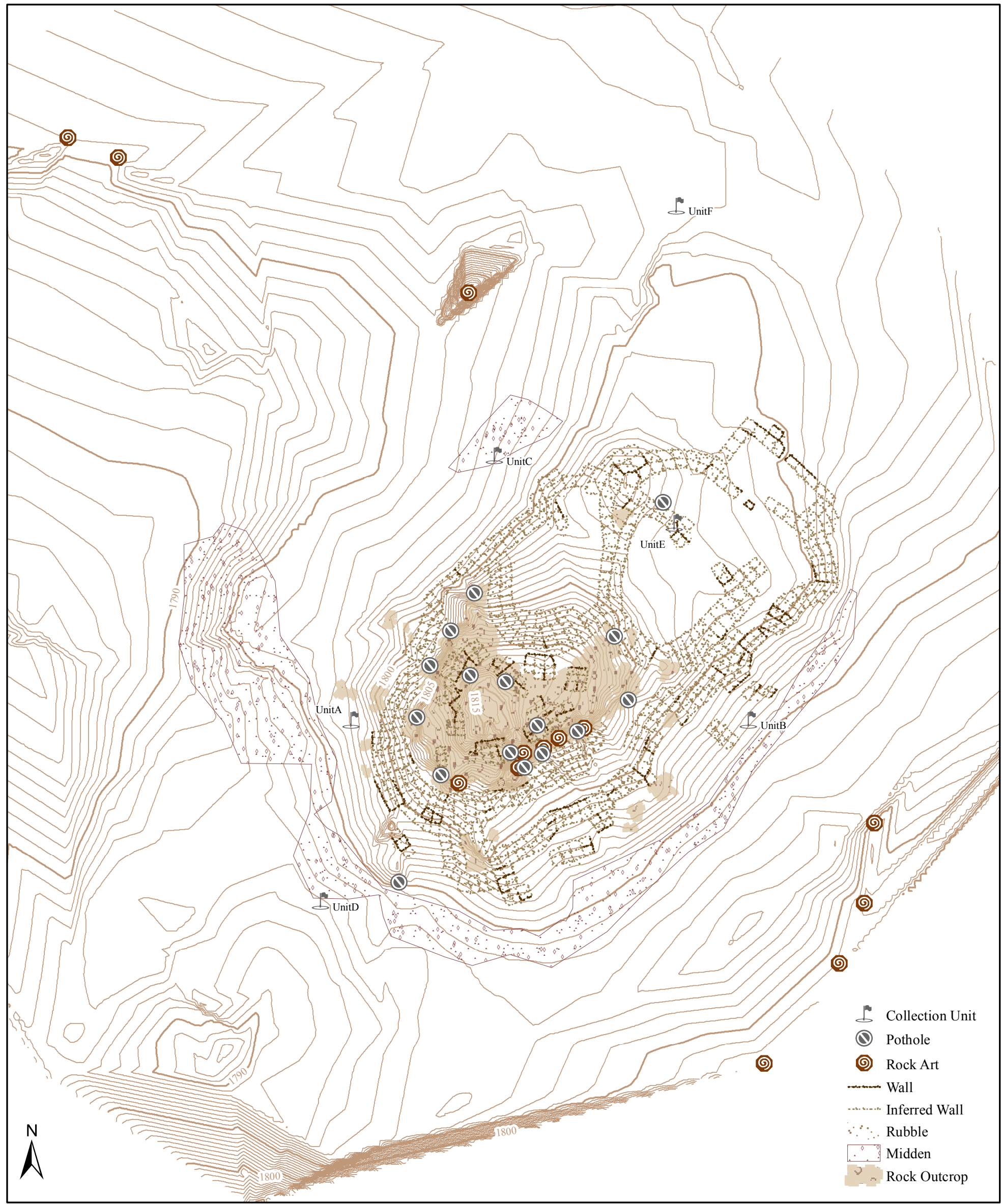




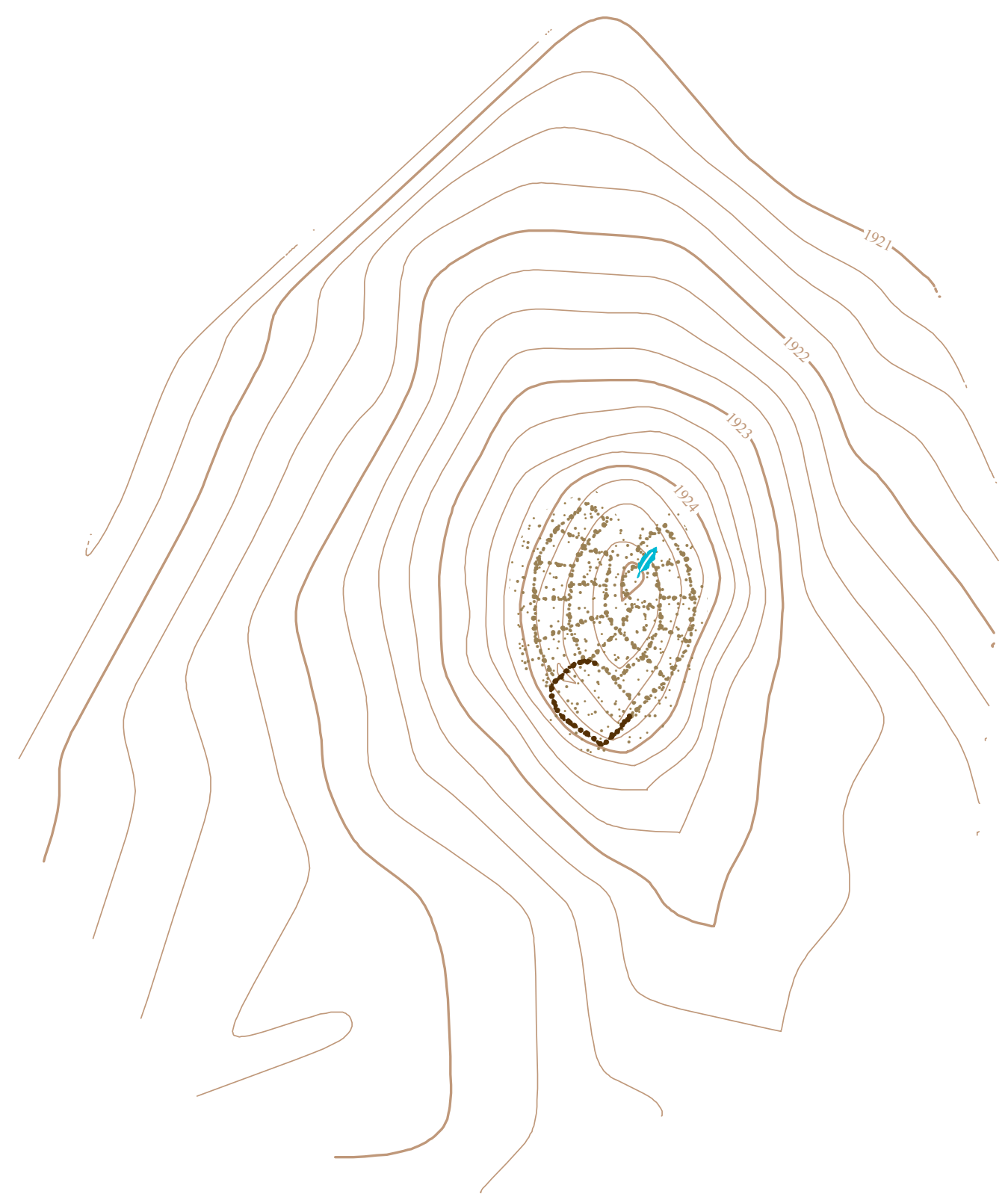




\section{Truskovi}

NA1132

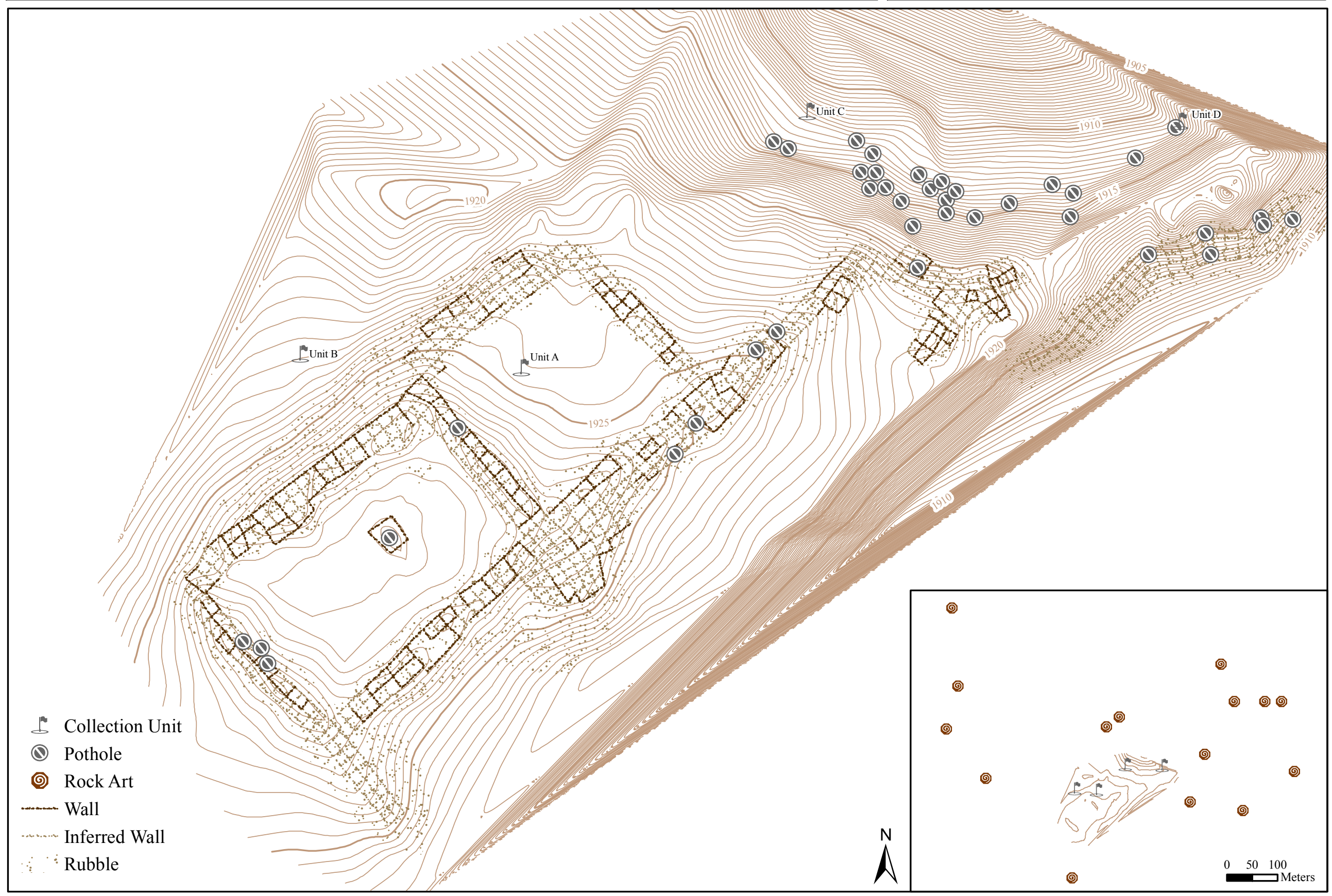




\section{Tsakpahu}

NA1039

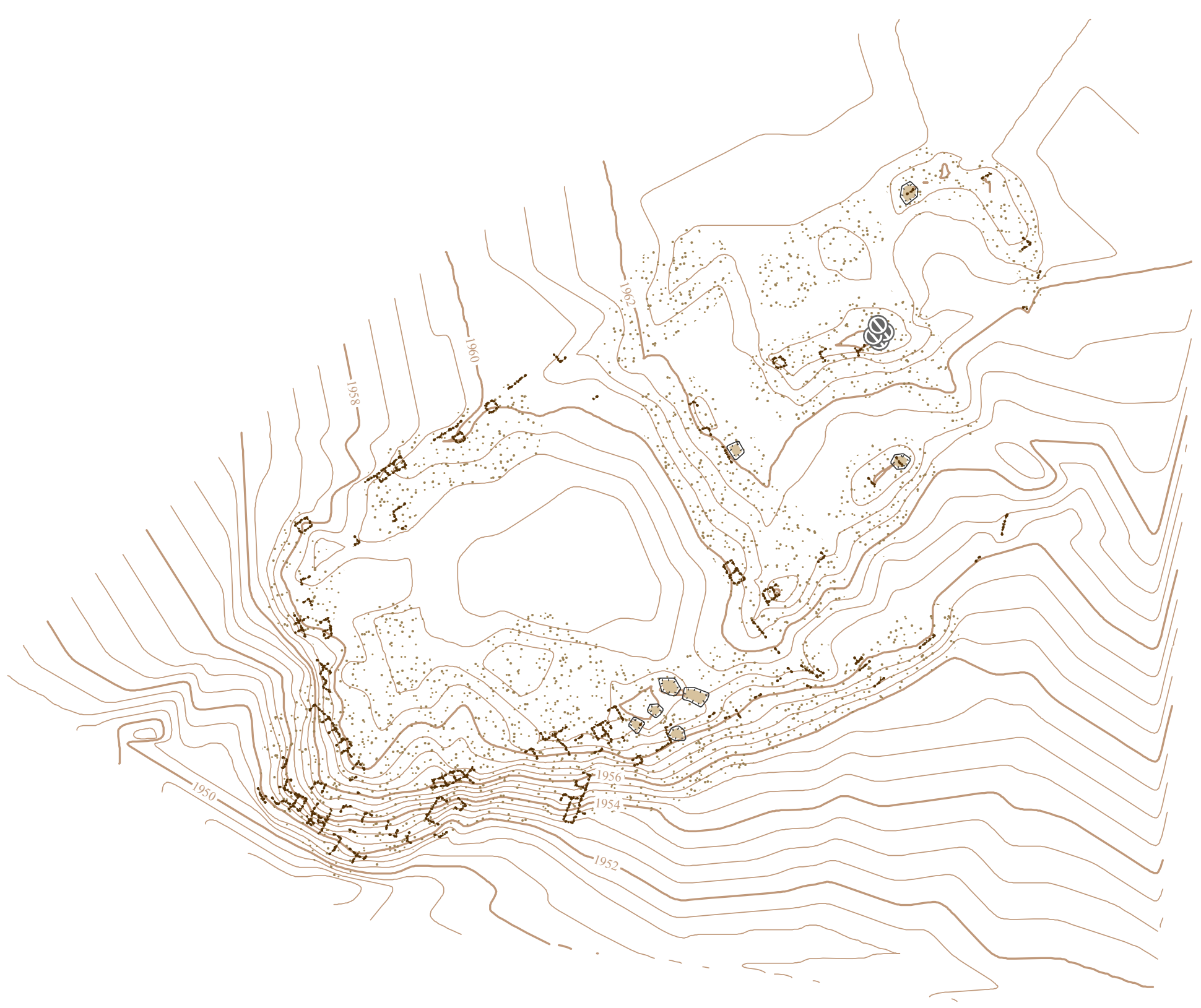

(a) Pothole Wall

Rubble

Depression 


\section{Tsaqkeni \\ NA30662}

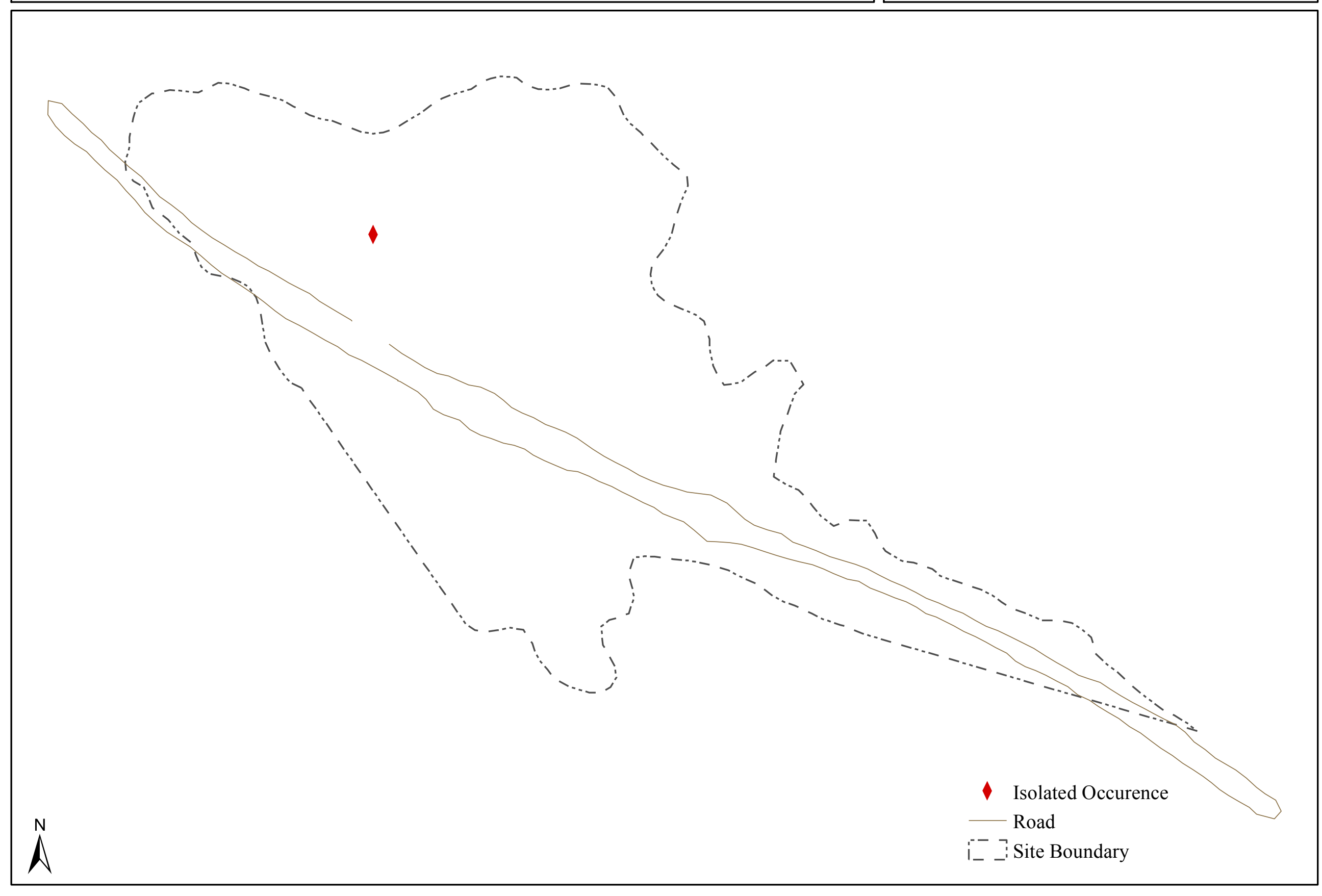




\begin{tabular}{|l|l}
\hline Tukinobi & NA30171 \\
\hline
\end{tabular}

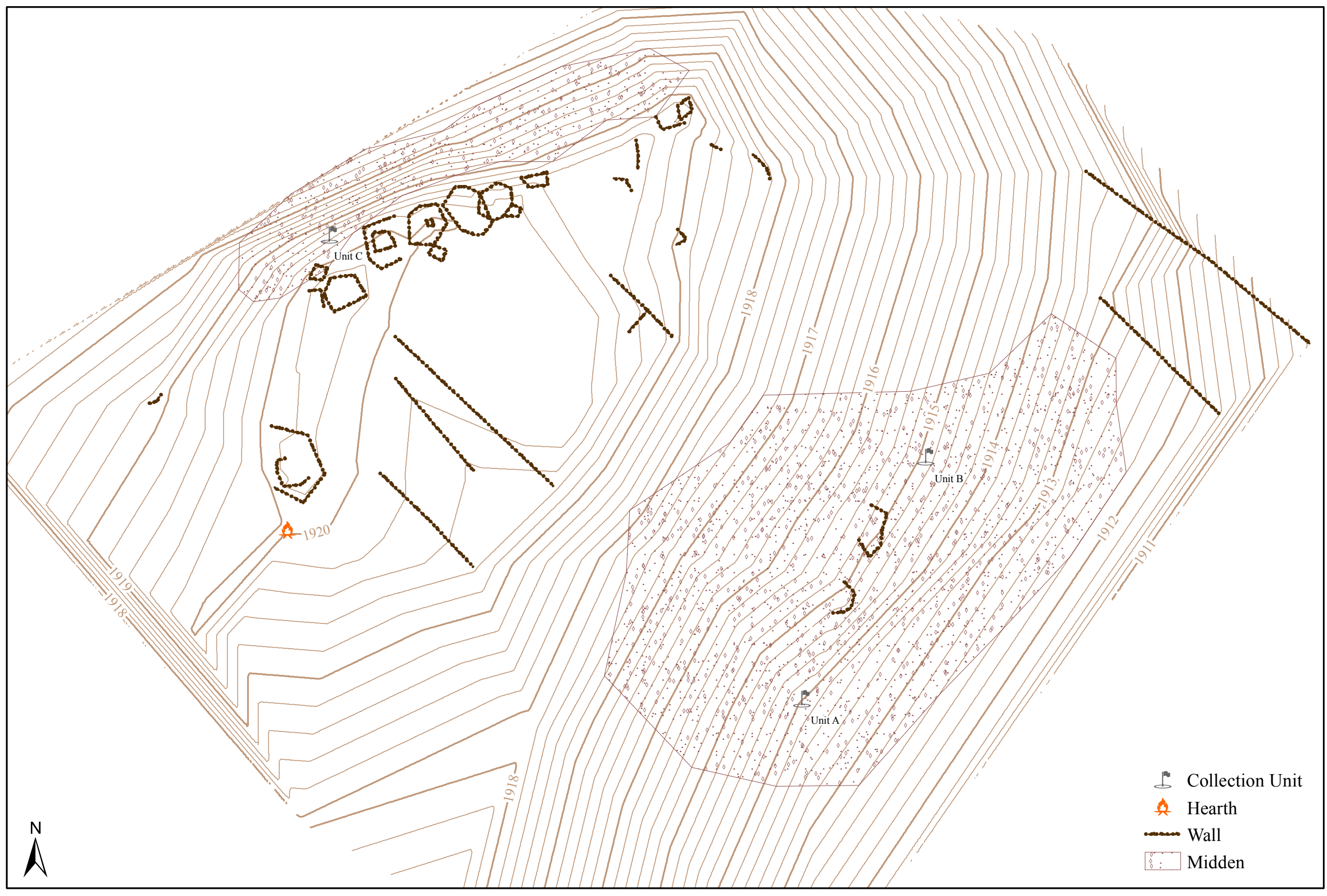




\section{Umtaqa}

NA29406

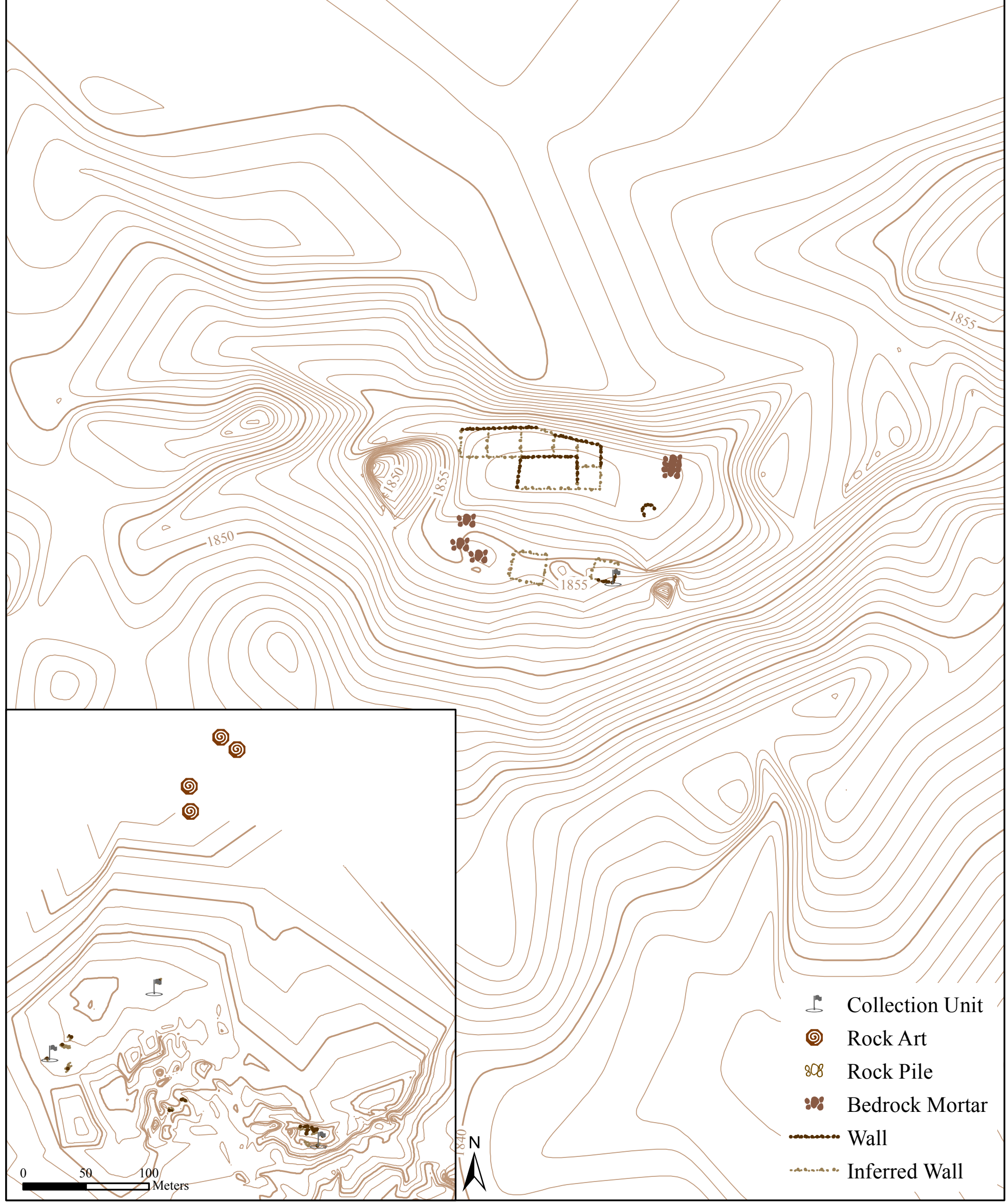

$\begin{array}{lll}0 & 10 & 20 \\ & \end{array}$ 


\section{White Ruin Canyon \\ NA30772}

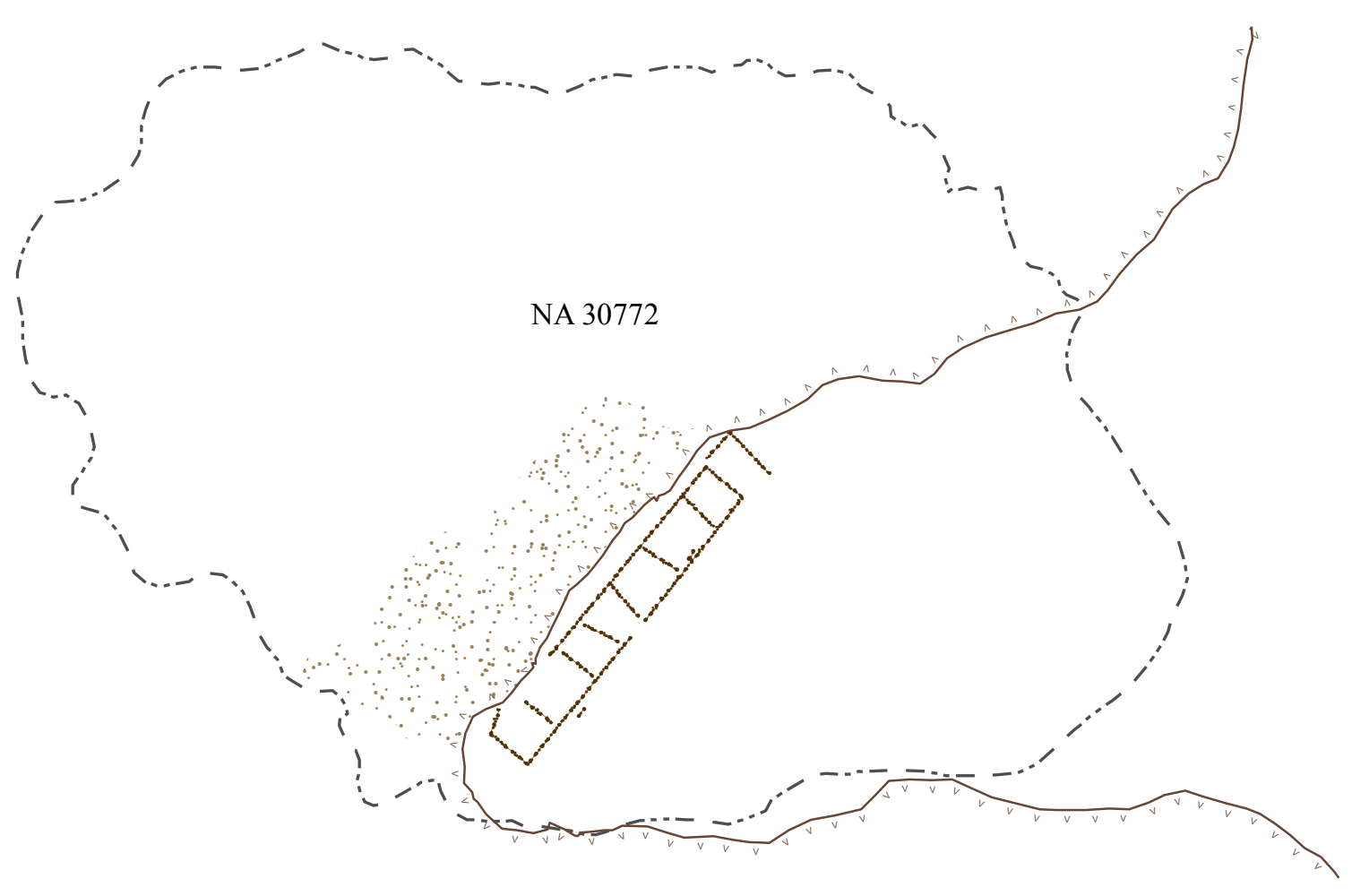

־ Cliff Edge

Wall

Rubble

i.- Site Boundary 


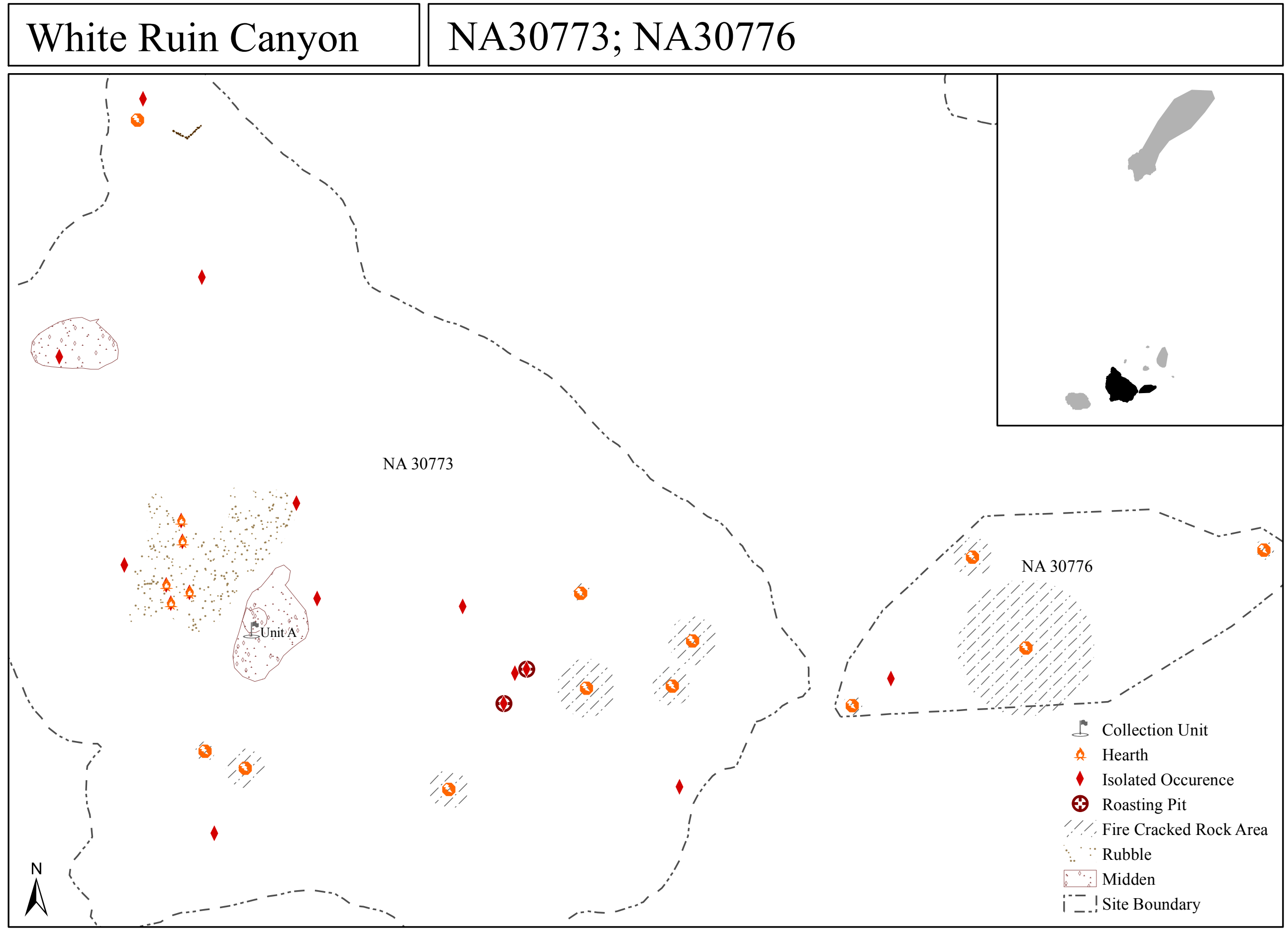




\section{\begin{tabular}{l|l} 
White Ruin Canyon & NA30775; NA30808 - NA30810
\end{tabular}}

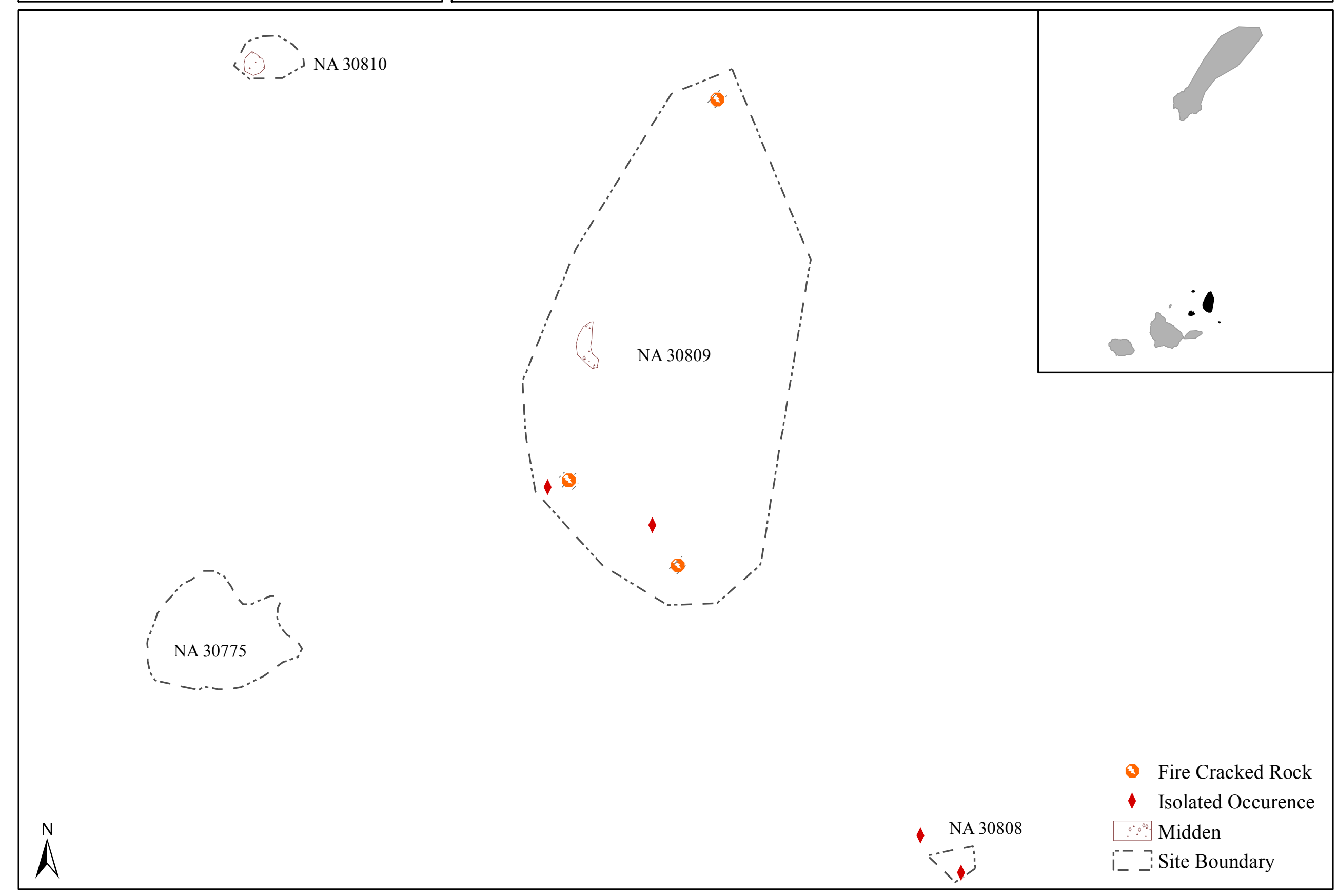




\section{White Ruin Canyon \\ NA30811}

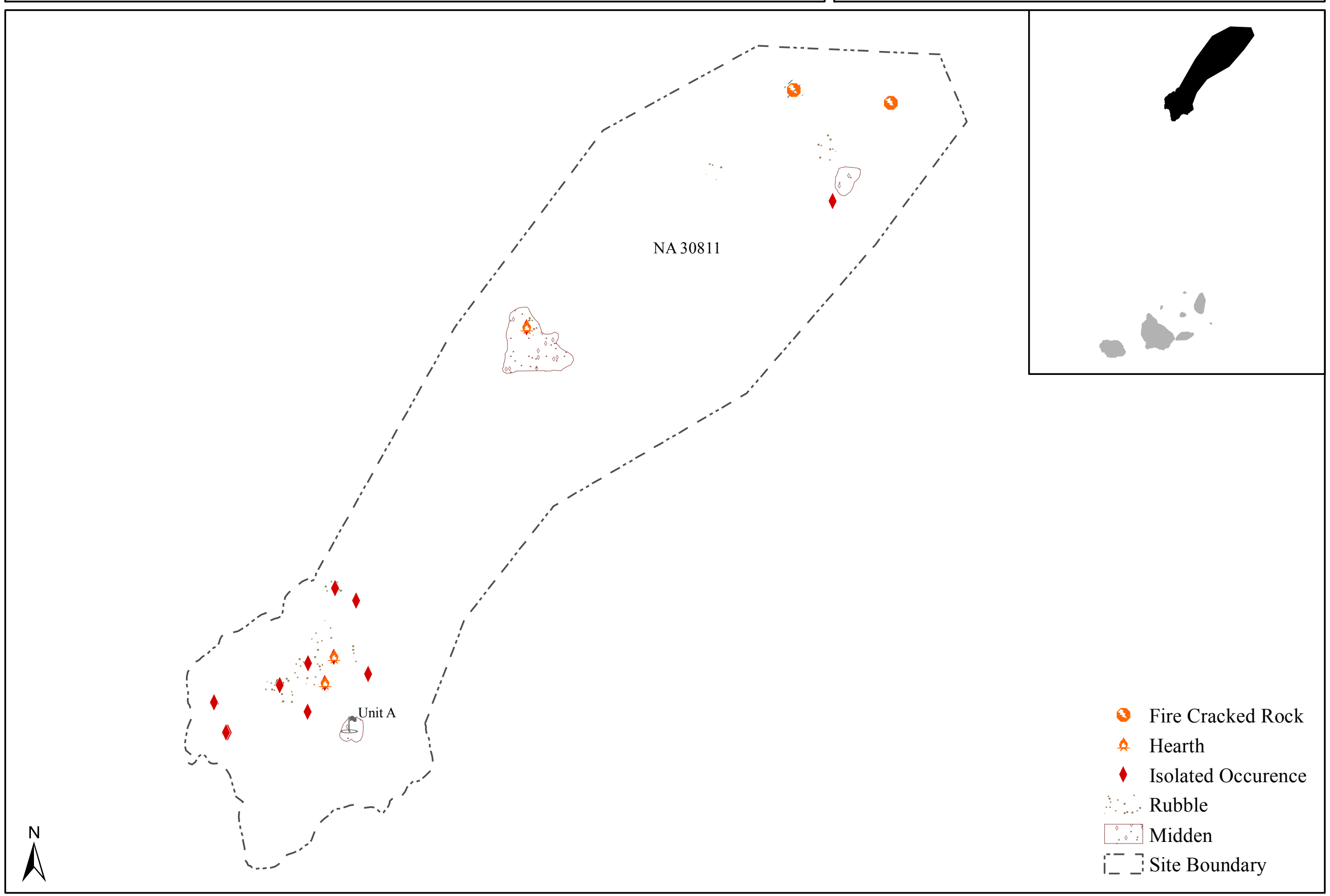




\section{White Spring \\ NA28334}

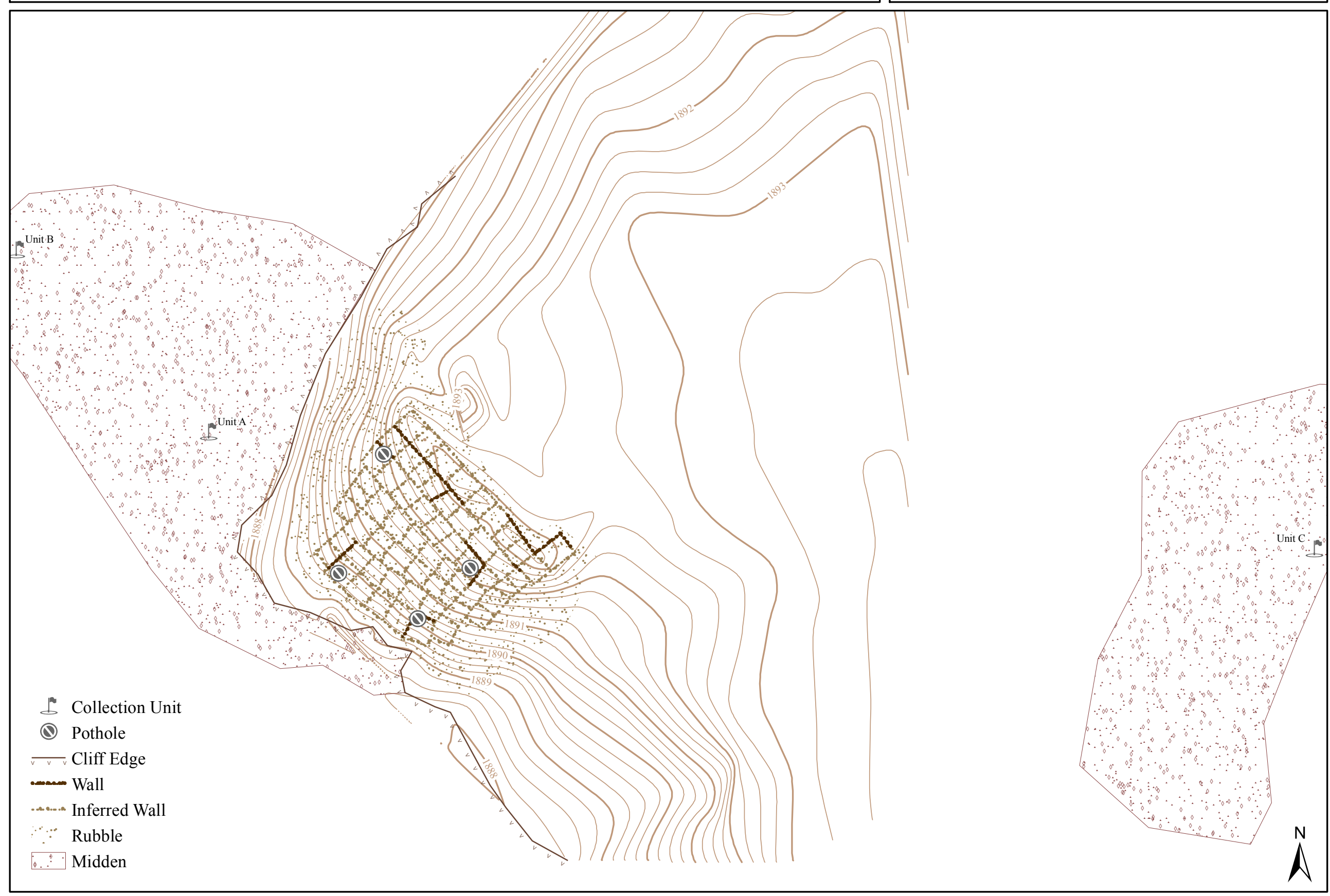




\section{Appendix B. Symbology Catalog}

\begin{tabular}{|c|c|c|c|c|c|c|c|c|c|c|c|}
\hline \multicolumn{12}{|c|}{ Symbology Catalog } \\
\hline & Type & RGB & HSL & CYMK & \#RGBA & RGB & HSL & CYMK & Label & Size & Offset \\
\hline \multicolumn{12}{|c|}{ Points } \\
\hline 1 & $\begin{array}{l}\text { Isolated } \\
\text { Occurrence }\end{array}$ & $212,0,0$ & $0,255,106$ & $0,100,100,17$ & $\mathrm{~d} 40000 \mathrm{ff}$ & $0,0,0$ & $0,0,0$ & $0,0,0,100$ & $\mathrm{n} / \mathrm{a}$ & $10 \mathrm{pt}$ & $\mathrm{n} / \mathrm{a}$ \\
\hline 2 & Cist & $140,81,10$ & $23,221,75$ & $0,42,93,45$ & $8 \mathrm{c} 510 \mathrm{aff}$ & $0,0,0$ & $0,0,0$ & $0,0,0,100$ & $\mathrm{n} / \mathrm{a}$ & $10 \mathrm{pt}$ & $\mathrm{n} / \mathrm{a}$ \\
\hline 3 & Upright Slab & $140,81,10$ & $23,221,75$ & $0,42,93,45$ & $8 \mathrm{c} 510 \mathrm{aff}$ & $0,0,0$ & $0,0,0$ & $0,0,0,100$ & $\mathrm{n} / \mathrm{a}$ & $10 \mathrm{pt}$ & $\mathrm{n} / \mathrm{a}$ \\
\hline 4 & $\begin{array}{l}\text { Collection } \\
\text { Unit }\end{array}$ & $102,102,102$ & $0,0,102$ & $0,0,0,60$ & $666666 \mathrm{ff}$ & $0,0,0$ & $0,0,0$ & $0,0,0,100$ & $\begin{array}{l}\text { White } \\
\text { halo 1pt }\end{array}$ & $10 \mathrm{pt}$ & $\mathrm{X} 0, \mathrm{Y} 3$ \\
\hline 5 & Pothole & $102,102,102$ & $0,0,102$ & $0,0,0,60$ & $666666 \mathrm{ff}$ & $0,0,0$ & $0,0,0$ & $0,0,0,100$ & $\mathrm{n} / \mathrm{a}$ & $10 \mathrm{pt}$ & $\mathrm{n} / \mathrm{a}$ \\
\hline 6 & Hearth & $255,102,0$ & $17,255,128$ & $0,60,100,0$ & ff6600ff & $0,0,0$ & $0,0,0$ & $0,0,0,100$ & $\mathrm{n} / \mathrm{a}$ & $10 \mathrm{pt}$ & $\mathrm{n} / \mathrm{a}$ \\
\hline 7 & $\begin{array}{l}\text { Fire Cracked } \\
\text { Rock }\end{array}$ & $255,102,0$ & $71,255,128$ & $0,60,100,0$ & ff6600ff & $0,0,0$ & $0,0,0$ & $0,0,0,100$ & $\mathrm{n} / \mathrm{a}$ & $10 \mathrm{pt}$ & $\mathrm{n} / \mathrm{a}$ \\
\hline 8 & Rock Art & $127,59,8$ & $18,225,68$ & $0,54,94,50$ & $7 \mathrm{f} 3 \mathrm{~b} 08 \mathrm{ff}$ & $0,0,0$ & $0,0,0$ & $0,0,0,100$ & $\mathrm{n} / \mathrm{a}$ & $10 \mathrm{pt}$ & $\mathrm{n} / \mathrm{a}$ \\
\hline 9 & Shrine & $0,185,212$ & $133,255,106$ & $100,13,0,17$ & 00b9d4f & $0,0,0$ & $0,0,0$ & $0,0,0,100$ & $\mathrm{n} / \mathrm{a}$ & $10 \mathrm{pt}$ & $\mathrm{X} 3, \mathrm{Y} 4$ \\
\hline 10 & Pithouse & $130,0,0$ & $0,255,65$ & $0,100,100,49$ & $820000 \mathrm{f}$ & $0,0,0$ & $0,0,0$ & $0,0,0,100$ & $\mathrm{n} / \mathrm{a}$ & $10 \mathrm{pt}$ & $\mathrm{n} / \mathrm{a}$ \\
\hline \multicolumn{12}{|c|}{ Lines } \\
\hline 11 & Boundary & $78,78,78$ & $0,0,31$ & $0,0,0,69$ & $\mathrm{n} / \mathrm{a}$ & $78,78,78$ & $0,0,31$ & $0,0,0,69$ & $\mathrm{n} / \mathrm{a}$ & $1 \mathrm{pt}$ & $\mathrm{n} / \mathrm{a}$ \\
\hline 12 & Trail & $138,112,69$ & $37,50,54$ & $46,56,73$ & $\mathrm{n} / \mathrm{a}$ & $104,104,104$ & $0,0,41$ & $0,0,0,59$ & $\mathrm{n} / \mathrm{a}$ & $.5 \mathrm{pt}$ & $\mathrm{n} / \mathrm{a}$ \\
\hline 13 & Wall & $84,48,5$ & $33,94,33$ & $67,81,98,0$ & $\mathrm{n} / \mathrm{a}$ & $0,0,0$ & $0,0,0$ & $0,0,0,100$ & $\mathrm{n} / \mathrm{a}$ & $1 \mathrm{pt}$ & $\begin{array}{l}\text { from - } \\
0.25 \text { to } \\
025\end{array}$ \\
\hline 14 & Inferred Wall & $153,128,84$ & $38,45,60$ & $19,24,39,0$ & $\mathrm{n} / \mathrm{a}$ & $130,130,130$ & $0,0,51$ & $0,0,0,49$ & $\mathrm{n} / \mathrm{a}$ & $1 \mathrm{pt}$ & $\begin{array}{l}\text { from - } \\
0.25 \text { to } \\
025\end{array}$ \\
\hline 15 & Cliff Edge & $105,69,52$ & $19,50,41$ & $59,73,80,0$ & $\mathrm{n} / \mathrm{a}$ & $0,0,0$ & $0,0,0$ & $0,0,0,100$ & $\mathrm{n} / \mathrm{a}$ & $3 \mathrm{pt}$ & $\mathrm{n} / \mathrm{a}$ \\
\hline 16 & Contours & $191,152,122$ & $26,36,75$ & $25,40,52,0$ & $\mathrm{n} / \mathrm{a}$ & $178,178,178$ & $0,0,61$ & $0,0,0,30$ & $\begin{array}{l}\text { White } \\
\text { halo } 2 \mathrm{pt}\end{array}$ & $\begin{array}{l}.5 \mathrm{pt} \text { and } \\
1 \mathrm{pt}\end{array}$ & $\mathrm{n} / \mathrm{a}$ \\
\hline \multicolumn{12}{|c|}{ Polygons } \\
\hline 17 & Bedrock & $\begin{array}{l}215,194,158 \\
\& 137,90,68\end{array}$ & $\begin{array}{l}38,27,84 \& 19 \\
50,54\end{array}$ & $\begin{array}{l}16,24,29,0 \& \\
137,90,68\end{array}$ & $\mathrm{n} / \mathrm{a}$ & $\begin{array}{l}204,204,204 \& \\
78,78,78\end{array}$ & $\begin{array}{l}0,0,80 \& 0 \\
0,31\end{array}$ & $\begin{array}{l}0,0,0,30 \& \\
0,0,0,69\end{array}$ & $\mathrm{n} / \mathrm{a}$ & $\mathrm{n} / \mathrm{a}$ & $\mathrm{n} / \mathrm{a}$ \\
\hline 18 & Ash Stain & $130,130,130$ & $0,0,51$ & $0,0,0,49$ & $\mathrm{n} / \mathrm{a}$ & $130,130,130$ & $0,0,51$ & $0,0,0,49$ & $\mathrm{n} / \mathrm{a}$ & $\mathrm{n} / \mathrm{a}$ & $\mathrm{n} / \mathrm{a}$ \\
\hline 19 & Midden & $137,68,68$ & $0,50,54$ & $46,73,73,0$ & $\mathrm{n} / \mathrm{a}$ & $78,78,78$ & $0,0,31$ & $0,0,0,69$ & $\mathrm{n} / \mathrm{a}$ & $\mathrm{n} / \mathrm{a}$ & $\mathrm{n} / \mathrm{a}$ \\
\hline 20 & Rubble & $153,128,84$ & $38,45,60$ & $40,50,67,0$ & $\mathrm{n} / \mathrm{a}$ & $104,104,104$ & $0,0,70$ & $0,0,0,59$ & $\mathrm{n} / \mathrm{a}$ & $\mathrm{n} / \mathrm{a}$ & $\mathrm{n} / \mathrm{a}$ \\
\hline 21 & Reservoir & $\begin{array}{l}0,197,255 \& \\
235,215,183\end{array}$ & $\begin{array}{l}194,100,100 \& \\
35,22,92\end{array}$ & $\begin{array}{l}100,24,0,0 \& \\
8,16,28,0\end{array}$ & $\mathrm{n} / \mathrm{a}$ & $\begin{array}{l}204,204,204 \& \\
78,78,78\end{array}$ & $\begin{array}{l}0,0,80 \& 0 \\
0,31\end{array}$ & $\begin{array}{l}0,0,0,20 \& \\
0,0,0,60\end{array}$ & $\mathrm{n} / \mathrm{a}$ & $\mathrm{n} / \mathrm{a}$ & $\mathrm{n} / \mathrm{a}$ \\
\hline
\end{tabular}




\section{Appendix C. Survey Results}

\begin{tabular}{|ccccc|}
\hline Survey Expected Answers & & & \\
\hline 1 Yes & $26 \mathrm{~A}$ & $51 \mathrm{~B}$ & $76 \mathrm{~B}$ & $101 \mathrm{~B}$ \\
$2 \mathrm{Any}$ & $27 \mathrm{~B}$ & $52 \mathrm{~A}$ & $77 \mathrm{~A}$ & $102 \mathrm{~A}$ \\
$3 \mathrm{Yes}$ & $28 \mathrm{~B}$ & $53 \mathrm{~A}$ & $78 \mathrm{~A}$ & $103 \mathrm{~B}$ \\
$4 \mathrm{n} / \mathrm{a}$ & $29 \mathrm{~B}$ & $54 \mathrm{~A}$ & $79 \mathrm{~A}$ & $104 \mathrm{~B}$ \\
$5 \mathrm{n} / \mathrm{a}$ & $30 \mathrm{~B}$ & $55 \mathrm{~A}$ & $80 \mathrm{~A}$ & $105 \mathrm{n} / \mathrm{a}$ \\
$6 \mathrm{n} / \mathrm{a}$ & $31 \mathrm{~A}$ & $56 \mathrm{~A}$ & $81 \mathrm{~B}$ & $106 \mathrm{n} / \mathrm{a}$ \\
$7 \mathrm{n} / \mathrm{a}$ & $32 \mathrm{~A}$ & $57 \mathrm{~B}$ & $82 \mathrm{~B}$ & $107 \mathrm{n} / \mathrm{a}$ \\
$8 \mathrm{Map} 2$ & $33 \mathrm{~B}$ & $58 \mathrm{~B}$ & $83 \mathrm{~B}$ & $108 \mathrm{n} / \mathrm{a}$ \\
$9 \mathrm{Map} 2$ & $34 \mathrm{~A}$ & $59 \mathrm{n} / \mathrm{a}$ & $84 \mathrm{~A}$ & $109 \mathrm{n} / \mathrm{a}$ \\
$10 \mathrm{Map} 1$ & $35 \mathrm{~A}$ & $60 \mathrm{n} / \mathrm{a}$ & $85 \mathrm{~A}$ & \\
$11 \mathrm{Map} 1$ & $36 \mathrm{~B}$ & $61 \mathrm{n} / \mathrm{a}$ & $86 \mathrm{~B}$ & \\
$12 \mathrm{Map} 2$ & $37 \mathrm{~B}$ & $62 \mathrm{n} / \mathrm{a}$ & $87 \mathrm{~A}$ & \\
$13 \mathrm{Map} 2$ & $38 \mathrm{~B}$ & $63 \mathrm{~B}$ & $88 \mathrm{~A}$ & \\
$14 \mathrm{Map} 2$ & $39 \mathrm{~B}$ & $64 \mathrm{~B}$ & $89 \mathrm{~A}$ & \\
$15 \mathrm{Map} 2$ & $40 \mathrm{~A}$ & $65 \mathrm{~A}$ & $90 \mathrm{~B}$ & \\
$16 \mathrm{Map} 2$ & $41 \mathrm{~A}$ & $66 \mathrm{~A}$ & $91 \mathrm{~B}$ & \\
$17 \mathrm{Map} 2$ & $42 \mathrm{~B}$ & $67 \mathrm{~A}$ & $92 \mathrm{~A}$ & \\
$18 \mathrm{n} / \mathrm{a}$ & $43 \mathrm{~A}$ & $68 \mathrm{~B}$ & $93 \mathrm{~A}$ & \\
$19 \mathrm{~B}$ & $44 \mathrm{~A}$ & $69 \mathrm{~B}$ & $94 \mathrm{~A}$ & \\
$20 \mathrm{~A}$ & $45 \mathrm{~A}$ & $70 \mathrm{~A}$ & $95 \mathrm{~A}$ & \\
$21 \mathrm{~B}$ & $46 \mathrm{~A}$ & $71 \mathrm{~B}$ & $96 \mathrm{~B}$ & \\
$22 \mathrm{~A}$ & $47 \mathrm{~A}$ & $72 \mathrm{~A}$ & \\
$23 \mathrm{~A}$ & $48 \mathrm{~B}$ & $73 \mathrm{~A}$ & $98 \mathrm{~A}$ & \\
$24 \mathrm{~A}$ & $49 \mathrm{~B}$ & $74 \mathrm{~A}$ & $99 \mathrm{~B}$ & \\
$25 \mathrm{~B}$ & $50 \mathrm{~B}$ & $75 \mathrm{~A}$ & $100 \mathrm{~A}$ & \\
\hline
\end{tabular}


Symbol Set Survey - A Cartographic Representation and Modeling of Hopi

Archaeology Sites

\section{Q1 I certify that I am 18 years or older.}

Answered: 29 Skipped: 0

Answer Choices

Yes

No - I can't participate in this survey(please exit survey)
Responses

$100.00 \%$

$0.00 \%$

\section{Q2 I certify I am currently enrolled in or majoring in one or more of the following disciplines.}

Answered: 29 Skipped: 0

Answer Choices

Anthropology

Archaeology

Environmental Sciences

History

Geographic Information Systems

None(please exit survey)
Responses

$3.45 \%$

$3.45 \%$

$3.45 \%$

$3.45 \%$

$93.10 \%$

$3.45 \%$

Total Respondents: 29

\section{Q3 I have read the above consent form and am willing to participate.}

Answered: 29 Skipped: 0

Answer Choices

Yes

No - I am not willing to participate (please exit survey)

\section{Responses}

$100.00 \%$

$0.00 \%$

\section{Q4 What is your current education level?}

Answered: 24 Skipped: 5

\begin{tabular}{|c|c|c|}
\hline Answer Choices & Respo & \\
\hline Freshman & $0.00 \%$ & 0 \\
\hline \multirow[t]{2}{*}{ Sophomore } & $0.00 \%$ & 0 \\
\hline & $4.17 \%$ & 1 \\
\hline \multicolumn{3}{|l|}{ Junior } \\
\hline Senior & $0.00 \%$ & 0 \\
\hline
\end{tabular}


Symbol Set Survey - A Cartographic Representation and Modeling of Hopi

Archaeology Sites

\begin{tabular}{l|l|l|}
\multicolumn{1}{|l|}{ Graduate } & $95.83 \%$ \\
\hline Total & Q5 How many GIS or cartography courses \\
have you taken? &
\end{tabular}

Answered: 24 Skipped: 5

\begin{tabular}{c|l|l}
\hline Answer Choices & Responses \\
\hline none & $0.00 \%$ & 0 \\
\hline 1 & $4.17 \%$ & 1 \\
\hline 2 & $4.17 \%$ \\
\hline 3 & $8.33 \%$ \\
\hline 4 & $0.00 \%$ \\
\hline $5+$ & $83.33 \%$ \\
\hline Total & 20 \\
\hline
\end{tabular}

\section{Q6 Please, rate yourself on the following map reading skills:}

\begin{tabular}{|c|c|c|c|c|c|c|c|c|}
\hline & Poor & $\begin{array}{l}\text { Below } \\
\text { Average }\end{array}$ & Average & $\begin{array}{l}\text { Above } \\
\text { Average }\end{array}$ & Excellent & N/A & Total & $\begin{array}{l}\text { Weighted } \\
\text { Average }\end{array}$ \\
\hline \multirow[t]{2}{*}{ How would you rate your map interpretation skills? } & $0.00 \%$ & $0.00 \%$ & $8.70 \%$ & $26.09 \%$ & $65.22 \%$ & $0.00 \%$ & & \\
\hline & 0 & 0 & 2 & 6 & 15 & 0 & 23 & 4.57 \\
\hline \multirow[t]{2}{*}{ How would you rate your spatial awareness skills? } & $0.00 \%$ & $0.00 \%$ & $0.00 \%$ & $39.13 \%$ & $60.87 \%$ & $0.00 \%$ & & \\
\hline & 0 & 0 & 0 & 9 & 14 & 0 & 23 & 4.61 \\
\hline \multirow[t]{2}{*}{ How would you rate your GIS skills? } & $0.00 \%$ & $0.00 \%$ & $4.35 \%$ & $34.78 \%$ & $60.87 \%$ & $0.00 \%$ & & \\
\hline & 0 & 0 & 1 & 8 & 14 & 0 & 23 & 4.57 \\
\hline \multirow{2}{*}{$\begin{array}{l}\text { How would you rate your ability to interpret symbols on a } \\
\text { map? }\end{array}$} & $0.00 \%$ & $0.00 \%$ & $4.35 \%$ & $26.09 \%$ & $69.57 \%$ & $0.00 \%$ & & \\
\hline & 0 & 0 & 1 & 6 & 16 & 0 & 23 & 4.65 \\
\hline \multirow[t]{2}{*}{ How would you rate your ability to read text on a map? } & $0.00 \%$ & $0.00 \%$ & $0.00 \%$ & $30.43 \%$ & $69.57 \%$ & $0.00 \%$ & & \\
\hline & 0 & 0 & 0 & 7 & 16 & 0 & 23 & 4.70 \\
\hline \multirow{2}{*}{$\begin{array}{l}\text { How would you rate your ability to understand a } \\
\text { topographic map? }\end{array}$} & $0.00 \%$ & $0.00 \%$ & $13.04 \%$ & $21.74 \%$ & $65.22 \%$ & $0.00 \%$ & & \\
\hline & 0 & 0 & 3 & 5 & 15 & 0 & 23 & 4.52 \\
\hline \multirow[t]{2}{*}{ How would you rate your artistic skills? } & $4.35 \%$ & $17.39 \%$ & $26.09 \%$ & $39.13 \%$ & $13.04 \%$ & $0.00 \%$ & & \\
\hline & 1 & 4 & 6 & 9 & 3 & 0 & 23 & 3.39 \\
\hline
\end{tabular}

\section{Q7 Please, rate how often you encounter maps:}

Answered: 23 Skipped: 6

\begin{tabular}{|r|r|r|}
\hline Never & Sometime & Often \\
\hline $0.00 \%$ & $30.43 \%$ & $17.39 \%$ \\
0 & 7 & 4 \\
\hline
\end{tabular}

\begin{tabular}{r|r} 
Frequently \\
\hline $34.78 \%$ \\
8
\end{tabular}

\begin{tabular}{|r|}
\hline Always \\
\hline $17.39 \%$ \\
4 \\
\hline
\end{tabular}

N/A
$\mathbf{0 . 0 0 \%}$
0

\begin{tabular}{|r|r|}
\hline Total & $\begin{array}{l}\text { Weighted } \\
\text { Average }\end{array}$ \\
\hline 23 & 3.39 \\
\hline
\end{tabular}


Symbol Set Survey - A Cartographic Representation and Modeling of Hopi

Archaeology Sites

How often do you use digital or online maps?

How often do you use the GPS on your mobile device?

How often do you use an interactive map (ex: Google Earth or

Bing Maps)

How often are maps used in your classrooms?

How often do you use maps for your projects or reports?

How often do you create maps?

\begin{tabular}{|c|c|c|c|c|c|c|c|}
\hline $0.00 \%$ & $0.00 \%$ & $4.35 \%$ & $43.48 \%$ & $52.17 \%$ & $0.00 \%$ & & \\
\hline 0 & 0 & 1 & 10 & 12 & 0 & 23 & 4.48 \\
\hline $0.00 \%$ & $4.35 \%$ & $4.35 \%$ & $43.48 \%$ & $47.83 \%$ & $0.00 \%$ & & \\
\hline 0 & 1 & 1 & 10 & 11 & 0 & 23 & 4.35 \\
\hline $4.35 \%$ & $21.74 \%$ & $13.04 \%$ & $30.43 \%$ & $30.43 \%$ & $0.00 \%$ & & \\
\hline 1 & 5 & 3 & 7 & 7 & 0 & 23 & 3.61 \\
\hline $0.00 \%$ & $13.04 \%$ & $13.04 \%$ & $43.48 \%$ & $17.39 \%$ & $13.04 \%$ & & \\
\hline 0 & 3 & 3 & 10 & 4 & 3 & 23 & 3.75 \\
\hline $0.00 \%$ & $4.35 \%$ & $30.43 \%$ & $34.78 \%$ & $30.43 \%$ & $0.00 \%$ & & \\
\hline 0 & 1 & 7 & 8 & 7 & 0 & 23 & 3.91 \\
\hline $0.00 \%$ & $4.35 \%$ & $34.78 \%$ & $34.78 \%$ & $26.09 \%$ & $0.00 \%$ & & \\
\hline 0 & 1 & 8 & 8 & 6 & 0 & 23 & 3.83 \\
\hline
\end{tabular}

\section{Q8 Which map is more visually appealing?}

Answered: 22 Skipped: 7

\begin{tabular}{|c|c|c|}
\hline Answer Choices & Responses & \\
\hline Map 1 & $63.64 \%$ & 14 \\
\hline Map 2 & $31.82 \%$ & 7 \\
\hline Both & $0.00 \%$ & 0 \\
\hline Neither & $4.55 \%$ & 1 \\
\hline NA & $0.00 \%$ & 0 \\
\hline Total & & 22 \\
\hline
\end{tabular}

\section{Q9 Which map is more legible?}

Answered: $21 \quad$ Skipped: 8

\begin{tabular}{|c|c|c|}
\hline Answer Choices & Respon & \\
\hline Map 1 & $38.10 \%$ & 8 \\
\hline Map 2 & $38.10 \%$ & 8 \\
\hline Both & $14.29 \%$ & 3 \\
\hline Neither & $9.52 \%$ & 2 \\
\hline NA & $0.00 \%$ & 0 \\
\hline Total & & 21 \\
\hline
\end{tabular}

\section{Q10 Which map is more visually appealing}

Answered: $21 \quad$ Skipped: 8

\begin{tabular}{|} 
Answer Cho \\
\hline Map 1 \\
\hline Map 2 \\
\hline Both
\end{tabular}


Symbol Set Survey - A Cartographic Representation and Modeling of Hopi

Archaeology Sites

\begin{tabular}{l|l|} 
Neither & $0.00 \%$ \\
\hline NA & $0.00 \%$ \\
\hline Total & \\
\hline 21 \\
\hline
\end{tabular}

\section{Q11 Which map is more legible?}

Answered: 21 Skipped: 8

\begin{tabular}{|c|c|c|}
\hline Answer Choices & Responses & \\
\hline Map 1 & $80.95 \%$ & 17 \\
\hline Map 2 & $9.52 \%$ & 2 \\
\hline Both & $4.76 \%$ & 1 \\
\hline Neither & $4.76 \%$ & 1 \\
\hline NA & $0.00 \%$ & 0 \\
\hline Total & & 21 \\
\hline
\end{tabular}

\section{Q12 Which map is more visually appealing?}

Answered: 20 Skipped: 9

\begin{tabular}{|c|c|c|}
\hline Answer Choices & Respons & \\
\hline Map 1 & $50.00 \%$ & 10 \\
\hline Map 2 & $35.00 \%$ & 7 \\
\hline Both & $5.00 \%$ & 1 \\
\hline Neither & $10.00 \%$ & 2 \\
\hline NA & $0.00 \%$ & 0 \\
\hline Total & & 20 \\
\hline
\end{tabular}

\section{Q13 Which map is more legible?}

Answered: 20 Skipped: 9

\begin{tabular}{|c|c|c|}
\hline Answer Choices & Responses & \\
\hline Map 1 & $30.00 \%$ & 6 \\
\hline Map 2 & $50.00 \%$ & 10 \\
\hline Both & $15.00 \%$ & 3 \\
\hline Neither & $5.00 \%$ & 1 \\
\hline NA & $0.00 \%$ & 0 \\
\hline Total & & 20 \\
\hline
\end{tabular}

\section{Q14 Which map is more visually appealing?}


Symbol Set Survey - A Cartographic Representation and Modeling of Hopi

Archaeology Sites

\begin{tabular}{|c|l|}
\hline Answer Choices & Responses \\
\hline Map 1 & $\mathbf{6 0 . 0 0 \%}$ \\
\hline Map 2 & $\mathbf{4 0 . 0 0 \%}$ \\
\hline Both & $\mathbf{0 . 0 0 \%}$ \\
\hline Neither & $\mathbf{0 . 0 0 \%}$ \\
\hline NA & $0.00 \%$ \\
\hline Total & 0 \\
\hline
\end{tabular}

\section{Q15 Which map is more legible?}

Answered: 20 Skipped: 9

\begin{tabular}{|l|l|}
\hline Answer Choices & Responses \\
\hline Map 1 & $45.00 \%$ \\
\hline Map 2 & $45.00 \%$ \\
\hline Both & $5.00 \%$ \\
\hline Neither & $0.00 \%$ \\
\hline NA & $5.00 \%$ \\
\hline Total & \\
\hline & Q16 Which map is more visually appealing?
\end{tabular}

Answered: 20 Skipped: 9

\begin{tabular}{|c|c|c|}
\hline Answer Choices & Responses & \\
\hline Map 1 & $35.00 \%$ & 7 \\
\hline Map 2 & $60.00 \%$ & 12 \\
\hline Both & $5.00 \%$ & 1 \\
\hline Neither & $0.00 \%$ & 0 \\
\hline NA & $0.00 \%$ & 0 \\
\hline Total & & 20 \\
\hline
\end{tabular}

\section{Q17 Which map is more legible?}

Answered: 19 Skipped: 10

Answer Choices

Map 1

Map 2

Both

Neither

NA

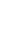

Responses

$31.58 \%$

$57.89 \%$

$5.26 \%$

$5.26 \%$

$0.00 \%$ 
Symbol Set Survey - A Cartographic Representation and Modeling of Hopi

Archaeology Sites

\section{Q18 Do you have any comments or suggestions for improvement about the previous maps?}

Answered: 10 Skipped: 19

Q19 Which symbol represents a shrine?

Answered: 19 Skipped: 10

Answer Choices

Symbol A

Symbol B

Total
Responses

$84.21 \%$

$15.79 \%$

Q20 Which symbol represents rock art?

Answered: 19 Skipped: 10

Answer Choices

Symbol A

Symbol B

Total
Responses

$21.05 \%$

$78.95 \%$
15

\section{Q21 Which symbol represents a cliff edge?}

Answered: 18 Skipped: 11

Answer Choices

Symbol A

Symbol B

Total
Responses

$44.44 \%$

$55.56 \%$

Q22 Which symbol represents midden?

Answered: 18 Skipped: 11

Answer Choices
Symbol A
Symbol B
Total

Responses

$55.56 \%$

10

$44.44 \%$

Q23 Which symbol represents a collection unit? 
Symbol Set Survey - A Cartographic Representation and Modeling of Hopi

Archaeology Sites

Answered: 19 Skipped: 10

\begin{tabular}{|l|l|}
\hline \multicolumn{1}{|l|}{$\begin{array}{l}\text { Answer Choices } \\
\text { Symbol A }\end{array}$} & Responses \\
\hline \multicolumn{1}{|l}{ Symbol B } & $84.21 \%$ \\
\hline Total & $15.79 \%$ \\
\hline & Q24 Which symbol represents a hearth?
\end{tabular}

Answered: 17 Skipped: 12

Answer Choices

Symbol A

Symbol B

Total
Responses

$64.71 \%$

$35.29 \%$

11

6

\section{Q25 Which symbol represents bedrock?}

Answered: 19 Skipped: 10

\begin{tabular}{l} 
Answer Choices \\
Symbol A \\
\hline Symbol B \\
\hline Total
\end{tabular}

Responses

$73.68 \%$

14

$26.32 \%$

\section{Q26 Which symbol represents a trail?}

Answered: 19 Skipped: 10

\begin{tabular}{|c|}
\hline Answer Choices \\
\hline Symbol A \\
\hline Symbol B \\
\hline
\end{tabular}

Responses

$21.05 \%$

Q27 Which symbol represents a pithouse?

Answered: 19 Skipped: 10

\begin{tabular}{|c|}
\hline Answer Choices \\
\hline Symbol A \\
\hline Symbol B \\
\hline Total
\end{tabular}


Symbol Set Survey - A Cartographic Representation and Modeling of Hopi

Archaeology Sites

\begin{tabular}{|l|l|}
\hline Answer Choices & Responses \\
\hline \multicolumn{1}{|l}{ Symbol A } & $31.58 \%$ \\
\hline \multicolumn{1}{|c|}{ Symbol B } & $68.42 \%$ \\
\hline Total & \\
\hline \multicolumn{2}{|l}{ Q29 Which symbol represents a cist? }
\end{tabular}

Answered: 16 Skipped: 13

Answer Choices

Symbol A

Symbol B

Total
Responses

$68.75 \%$

$31.25 \%$

11

\section{Q30 Which symbol represents ash stain?}

Answered: 18 Skipped: 11

Answer Choices

Symbol A

Symbol B

Total

Responses

$5.56 \%$

$94.44 \%$

\section{Q31 Which symbol represents a survey site boundary?}

Answered: 18 Skipped: 11

\begin{tabular}{l} 
Answer Choices \\
Symbol A \\
\hline Symbol B \\
\hline Total \\
\hline
\end{tabular}

Responses

$94.44 \%$

$5.56 \%$

\section{Q32 Which symbol represents a stone wall?}

Answered: 16 Skipped: 13

\begin{tabular}{l} 
Answer Choices \\
Symbol A \\
Symbol B \\
\hline Total
\end{tabular}

Responses

$62.50 \%$

$37.50 \%$

\section{Q33 Which symbol represents a pothole?}

Answered: 18 Skipped: 11 
Symbol Set Survey - A Cartographic Representation and Modeling of Hopi

Archaeology Sites

\begin{tabular}{|c|c|c|}
\hline Symbol A & $44.44 \%$ & 8 \\
\hline Symbol B & $55.56 \%$ & 10 \\
\hline Total & & 18 \\
\hline
\end{tabular}

\section{Q34 Which symbol represents rubble?}

Answered: 17 Skipped: 12

Answer Choices

Symbol A

Symbol B

Total

\section{Responses}

$88.24 \%$

$11.76 \%$

\section{Q35 Which symbol represents an inferred} stone wall?

Answered: 17 Skipped: 12

Answer Choices

Symbol A

Symbol B

Total
Responses

$94.12 \%$

$5.88 \%$

Q36 Which symbol represents fire cracked rock?

Answered: 18 Skipped: 11

Answer Choices

Symbol A

Symbol B
Responses

$11.11 \%$

$88.89 \%$

Q37 Which symbol represents an upright slab?

Answered: 15 Skipped: 14

\begin{tabular}{|c|}
\hline Answer Choices \\
\hline Symbol A \\
\hline Symbol B \\
\hline Total
\end{tabular}

Responses

$60.00 \%$

$40.00 \%$
9

6

15

\section{Q38 Which symbol represents an agricultural wall?}


Symbol Set Survey - A Cartographic Representation and Modeling of Hopi

Archaeology Sites

Answered: 16 Skipped: 13

Answer Choices

Symbol A

Symbol B

Total
Responses

$43.75 \%$

$56.25 \%$

Q39 Which symbol represents rock art?

Answered: 17 Skipped: 12

Answer Choices

Symbol A

Symbol B

Total
Responses

$88.24 \%$

$11.76 \%$

\section{Q40 Which symbol represents midden?}

Answered: 16 Skipped: 13

Answer Choices

Symbol A

Symbol B

Total
Responses

$93.75 \%$

$6.25 \%$

1

\section{Q41 Which symbol represents a hearth?}

Answered: 16 Skipped: 13

Answer Choices

Symbol A

Symbol B

Total
Responses

$62.50 \%$

$37.50 \%$

\section{Q42 Which symbol represents a trail?}

Answered: 17 Skipped: 12

Answer Choices

Symbol A

Symbol B

\section{Responses}

$0.00 \%$

$100.00 \%$

Q43 Which symbol represents an isolated occurrence? 
Symbol Set Survey - A Cartographic Representation and Modeling of Hopi

Archaeology Sites

Answer Choices

Symbol A

Symbol B

Total

\section{Responses}

$76.47 \%$

$23.53 \%$

\section{Q44 Which symbol represents a shrine?}

Answered: 16 Skipped: 13

\begin{tabular}{|l|l}
\hline Answer Choices & \multicolumn{1}{l}{ Responses } \\
\hline Symbol A & $25.00 \%$ \\
\hline Symbol B & $75.00 \%$ \\
\hline Total & Q45 Which symbol represents a ciff edge? \\
\hline
\end{tabular}

Answered: 16 Skipped: 13

\begin{tabular}{l} 
Answer Choices \\
Symbol A \\
\hline Symbol B \\
\hline Total
\end{tabular}

Responses

$81.25 \%$

$18.75 \%$

\section{Q46 Which symbol represents a collection unit?}

Answered: 17 Skipped: 12

\begin{tabular}{|c|c|c|}
\hline Answer Choices & Responses & \\
\hline Symbol A & $88.24 \%$ & 15 \\
\hline Symbol B & $11.76 \%$ & 2 \\
\hline Total & & 17 \\
\hline
\end{tabular}

\section{Q47 Which symbol represents bedrock?}

Answered: 17 Skipped: 12

\begin{tabular}{c} 
Answer Choices \\
\hline Symbol A \\
\hline Symbol B \\
\hline
\end{tabular}

\section{Responses}

$5.88 \%$

$94.12 \%$

\section{Q48 Which symbol represents a pithouse?}


Symbol Set Survey - A Cartographic Representation and Modeling of Hopi

Archaeology Sites

\begin{tabular}{|c|l|}
\hline Answer Choices & Responses \\
\hline Symbol A & $23.53 \%$ \\
\hline Symbol B & $\mathbf{7 6 . 4 7 \%}$ \\
\hline Total & \\
\hline
\end{tabular}

\section{Q49 Which symbol represents ash stain?}

Answered: 16 Skipped: 13

Answer Choices

Symbol A

Symbol B

Total

\begin{tabular}{lr} 
Responses & \\
\hline $\mathbf{1 2 . 5 0 \%}$ & 2 \\
\hline $\mathbf{8 7 . 5 0 \%}$ & 14 \\
\hline & 16 \\
\hline
\end{tabular}

Q50 Which symbol represents a stone wall?

Answered: 15 Skipped: 14

Answer Choices

Symbol A

Symbol B

Total

Responses

$26.67 \%$

$73.33 \%$

\section{Q51 Which symbol represents rubble?}

Answered: 15 Skipped: 14

\begin{tabular}{|c|}
\hline Answer Choices \\
\hline Symbol A \\
\hline Symbol B \\
\hline
\end{tabular}

Q52 Which symbol represents fire cracked rock?

Answered: 16 Skipped: 13

\begin{tabular}{|l|l|}
\hline \multicolumn{1}{|l|}{ Answer Choices } & Responses \\
\hline \multicolumn{1}{|l|}{ Symbol A } & $93.75 \%$ \\
\hline Symbol B & $6.25 \%$ \\
\hline Total & Q53 Which symbol represents an \\
& agricultural wall? \\
\hline
\end{tabular}


Symbol Set Survey - A Cartographic Representation and Modeling of Hopi

Archaeology Sites

\begin{tabular}{|l|l|}
\hline Answer Choices & Responses \\
\hline \multicolumn{1}{|c|}{ Symbol A } & $43.75 \%$ \\
\hline \multicolumn{1}{|c|}{ Symbol B } & $56.25 \%$ \\
\hline Total & \\
\hline \multicolumn{2}{l}{ Q54 Which symbol represents a cist? }
\end{tabular}

Answered: 15 Skipped: 14

Answer Choices

Symbol A

Symbol B

Total

\section{Responses}

$33.33 \%$

$66.67 \%$

Q55 Which symbol represents a site survey boundary?

Answered: 15 Skipped: 14

Answer Choices
Symbol A
Symbol B
Total

\section{Q56 Which symbol represents a pothole?}

Answered: 16 Skipped: 13

Answer Choices
Symbol A
Symbol B
Total

Responses

$62.50 \%$

Q57 Which symbol represents an inferred stone wall?

Answered: 15 Skipped: 14

\begin{tabular}{c} 
Answer Choices \\
\hline Symbol A \\
\hline Symbol B \\
\hline Total
\end{tabular}

Responses
$0.00 \%$
$100.00 \%$


Symbol Set Survey - A Cartographic Representation and Modeling of Hopi

Archaeology Sites

Answered: 15 Skipped: 14

\begin{tabular}{|c|}
\hline Answer Choices \\
\hline Symbol A \\
\hline Symbol B \\
\hline Total \\
\hline
\end{tabular}

Responses

$66.67 \%$

10

$33.33 \%$

\section{Q59 How easy was it to interpret the symbols to what they represent?}

Answered: 17 Skipped: 12

\begin{tabular}{|l|l|}
\hline Answer Choices & Responses \\
\hline Easy & $0.00 \%$ \\
\hline Fair & $\mathbf{0 . 0 0 \%}$ \\
\hline Moderate & $\mathbf{5 8 . 8 2 \%}$ \\
\hline Difficult & $\mathbf{2 9 . 4 1 \%}$ \\
\hline Challenging & $\mathbf{1 1 . 7 6 \%}$ \\
\hline Total & \\
\hline
\end{tabular}

Q60 How cohesive was the symbol set?

Answered: 17 Skipped: 12

\begin{tabular}{|c|} 
Answer Choices \\
Very cohesive \\
\hline Fairly cohesive \\
Somewhat cohesive \\
Not cohesive
\end{tabular}

\begin{tabular}{|lc}
\hline Responses & \\
\hline $\mathbf{0 . 0 0 \%}$ & 0 \\
\hline $\mathbf{2 3 . 5 3 \%}$ & 4 \\
\hline $\mathbf{4 7 . 0 6 \%}$ & 8 \\
\hline $\mathbf{2 9 . 4 1 \%}$ & 5 \\
\hline & $\mathbf{1 7}$ \\
\hline
\end{tabular}

\section{Q61 How sure are you about your answers?}

Answered: 16 Skipped: 13

\begin{tabular}{|l|l|}
\hline Answer Choices & Responses \\
\hline Very sure & $12.50 \%$ \\
\hline Sure & $6.25 \%$ \\
\hline Fairly Sure & $31.25 \%$ \\
\hline Unsure & $37.50 \%$ \\
\hline Very unsure & $12.50 \%$ \\
\hline Total & \\
\hline
\end{tabular}


Symbol Set Survey - A Cartographic Representation and Modeling of Hopi

Archaeology Sites

like to provided about Symbol Set 1?

Answered: 4 Skipped: 25

\section{Q63 Which symbol represents a cist?}

Answered: 16 Skipped: 13

Answer Choices

Symbol A

Symbol B
Responses

$18.75 \%$

$81.25 \%$

Q64 Which symbol represents ash stain?

Answered: 17 Skipped: 12

Answer Choices

Symbol A

Symbol B

Total
Responses

$29.41 \%$

$70.59 \%$

12

Q65 Which symbol represents a survey site boundary?

Answered: 17 Skipped: 12

\begin{tabular}{l|l} 
Answer Choices & Responses \\
\hline Symbol A & $64.71 \%$ \\
\hline Symbol B & $35.29 \%$ \\
\hline Total & Q66 Which Symbol represents a Stone Wall? \\
\hline
\end{tabular}

Answered: 16 Skipped: 13

Answer Choices

Symbol A

Symbol B
Responses

$93.75 \%$

$6.25 \%$

Q67 Which symbol represents a pothole?

Answered: 17 Skipped: 12 
Symbol Set Survey - A Cartographic Representation and Modeling of Hopi

Archaeology Sites

\begin{tabular}{|l|l|}
\hline Symbol B & $70.59 \%$ \\
\hline Total & 12 \\
\hline
\end{tabular}

\section{Q68 Which symbol represents rubble?}

Answered: 16 Skipped: 13

\begin{tabular}{l} 
Answer Choices \\
Symbol A \\
Symbol B \\
\hline Total
\end{tabular}

Responses

$31.25 \%$

$68.75 \%$

Q69 Which symbol represents an inferred stone wall?

Answered: 16 Skipped: 13

\begin{tabular}{|c|} 
Answer Choices \\
\hline Symbol A \\
\hline Symbol B \\
\hline Total
\end{tabular}

Responses

$25.00 \%$

$75.00 \%$

Q70 Which symbol represents fire cracked rock?

Answered: 17 Skipped: 12

\begin{tabular}{|c|}
\hline Answer Choices \\
\hline Symbol A \\
\hline Symbol B \\
\hline Total \\
\hline
\end{tabular}

Responses

$76.47 \%$

$23.53 \%$

\section{Q71 Which symbol represents an upright slab?}

Answered: 16 Skipped: 13

\begin{tabular}{|l|l|}
\hline $\begin{array}{l}\text { Answer Choices } \\
\text { Symbol A }\end{array}$ & \multicolumn{1}{c}{ Responses } \\
\hline Symbol B & $100.00 \%$ \\
\hline Total & Q72 Which symbol represents an \\
& agricultural wall? \\
\hline
\end{tabular}


Symbol Set Survey - A Cartographic Representation and Modeling of Hopi

Archaeology Sites

\begin{tabular}{|l|l|}
\hline Answer Choices & Responses \\
\hline \multicolumn{1}{|c|}{ Symbol A } & $88.24 \%$ \\
\hline \multicolumn{1}{|c|}{ Symbol B } & $11.76 \%$ \\
\hline Total & \\
\hline & Q73 Which symbol represents a reservior?
\end{tabular}

Answered: 17 Skipped: 12

\begin{tabular}{|l|l|}
\hline $\begin{array}{l}\text { Answer Choices } \\
\text { Symbol A }\end{array}$ & Responses \\
\hline \multicolumn{1}{|c|}{ Symbol B } & $100.00 \%$ \\
\hline Total & $0.00 \%$ \\
\hline & \\
\hline & Q74 Which symbol represents a shrine?
\end{tabular}

Answered: 16 Skipped: 13

\begin{tabular}{|c|c|}
\hline Answer Choices & Responses \\
\hline Symbol A & $50.00 \%$ \\
\hline Symbol B & $50.00 \%$ \\
\hline Total & \\
\hline
\end{tabular}

Answered: 16 Skipped: 13

\begin{tabular}{|c|}
\hline Answer Choices \\
\hline Symbol A \\
\hline Symbol B \\
\hline Total
\end{tabular}

Q76 Which symbol represents a cliff edge?

Answered: 16 Skipped: 13

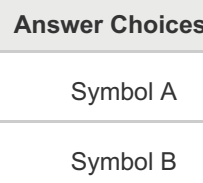

\section{Q77 Which symbol represents midden?}


Symbol Set Survey - A Cartographic Representation and Modeling of Hopi

Archaeology Sites

\begin{tabular}{|c|c|c|}
\hline Symbol A & $81.25 \%$ & 13 \\
\hline Symbol B & $18.75 \%$ & 3 \\
\hline Total & & 16 \\
\hline
\end{tabular}

\section{Q78 Which symbol represents a collection unit?}

Answered: 17 Skipped: 12

Answer Choices

Symbol A

Symbol B

Total
Responses

$82.35 \%$

$17.65 \%$

\section{Q79 Which symbol represents a hearth?}

Answer Choices

Symbol A

Symbol B

Total
Responses

$87.50 \%$

$12.50 \%$

14

2

\section{Q80 Which symbol represents bedrock?}

Answered: 17 Skipped: 12

Answer Choices

Symbol A

Symbol B

Total

\section{Responses}

$70.59 \%$

$29.41 \%$

\section{Q81 Which symbol represents a trail?}

Answered: 15 Skipped: 14

\begin{tabular}{l} 
Answer Choices \\
Symbol A \\
\hline Symbol B \\
Total
\end{tabular}

Responses

$6.67 \%$

1

$93.33 \%$

\section{Q82 Which symbol represents a pithouse?}

Answered: 16 Skipped: 13 
Symbol Set Survey - A Cartographic Representation and Modeling of Hopi

Archaeology Sites

\section{Q83 Which symbol represents an isolated occurrence?}

Answered: 16 Skipped: 13

Answer Choices

Symbol A

Symbol B

Total
Responses

$12.50 \%$

$87.50 \%$

Q84 Which symbol represents ash stain?

Answered: 15 Skipped: 14

Answer Choices

Symbol A

Symbol B

Total
Responses

$73.33 \%$

$26.67 \%$

Q85 Which symbol represents a stone wall?

Answered: 15 Skipped: 14

Answer Choices

Symbol A

Symbol B

Total

Responses

$93.33 \%$

$6.67 \%$

\section{Q86 Which symbol represents rubble?}

Answered: 15 Skipped: 14

\begin{tabular}{l} 
Answer Choices \\
Symbol A \\
\hline Symbol B \\
Total
\end{tabular}

Responses

$0.00 \%$

0

$100.00 \%$

15

\section{Q87 Which symbol represents fire cracked} rock?

Answered: 14 Skipped: 15 
Symbol Set Survey - A Cartographic Representation and Modeling of Hopi

Archaeology Sites

\begin{tabular}{|l|l|}
\hline Symbol A & $100.00 \%$ \\
\hline Symbol B & $0.00 \%$ \\
\hline Total & \\
\hline
\end{tabular}

\section{Q88 Which symbol represents an agricultural wall?}

Answer Choices

Symbol A

Symbol B

Total

\section{Responses}

$75.00 \%$

$25.00 \%$

\section{Q89 Which symbol represents a cist?}

Answered: 15 Skipped: 14

Answer Choices

Symbol A

Symbol B

Total
Responses

$100.00 \%$

$0.00 \%$
15

0

Q90 Which symbol represents a site survey boundary?

Answered: 15 Skipped: 14

Answer Choices

Symbol A

Symbol B

Total
Responses

$0.00 \%$

$100.00 \%$
0

Q91 Which symbol represents a pothole?

Answered: 15 Skipped: 14

Answer Choices

Responses

$33.33 \%$

5

$66.67 \%$

10

Q92 Which symbol represents an inferred stone wall? 
Symbol Set Survey - A Cartographic Representation and Modeling of Hopi

Archaeology Sites

\begin{tabular}{|c|l|}
\hline Answer Choices & Responses \\
\hline Symbol A & $93.33 \%$ \\
\hline Symbol B & $6.67 \%$ \\
\hline Total & \\
\hline
\end{tabular}

\section{Q93 Which symbol represents a reservoir?}

Answered: 16 Skipped: 13
Answer Choices

Symbol A

Symbol B

Total
Responses

$100.00 \%$

$0.00 \%$

\section{Q94 Which symbol represents an upright slab?}

Answered: 16 Skipped: 13
Answer Choices

Symbol A

Symbol B

Total
Responses

$100.00 \%$

$0.00 \%$

1


Symbol Set Survey - A Cartographic Representation and Modeling of Hopi

Archaeology Sites

\begin{tabular}{|l|l|} 
Symbol A & $6.67 \%$ \\
\hline Symbol B & $93.33 \%$ \\
\hline Total & \\
\hline
\end{tabular}

Q98 Which symbol represents a trail?

Answered: 15 Skipped: 14

\begin{tabular}{|c|c|c|}
\hline Answer Choices & Respons & \\
\hline Symbol A & $66.67 \%$ & 10 \\
\hline Symbol B & $33.33 \%$ & 5 \\
\hline Total & & 15 \\
\hline
\end{tabular}

\section{Q99 Which symbol represents an isolated occurrence?}

Answered: 15 Skipped: 14

\begin{tabular}{|c|c|c|}
\hline Answer Choices & Responses & \\
\hline Symbol A & $0.00 \%$ & 0 \\
\hline Symbol B & $100.00 \%$ & 15 \\
\hline Total & & 15 \\
\hline
\end{tabular}

\section{Q100 Which symbol represents a shrine?}

Answered: 15 Skipped: 14

Answer Choices

Symbol A

Symbol B

Total
Responses

$93.33 \%$

$6.67 \%$

\section{Q101 Which symbol represents a cliff edge?}

Answered: 15 Skipped: 14

\begin{tabular}{l} 
Answer Choices \\
Symbol A \\
\hline Symbol B \\
Total
\end{tabular}

Responses

$26.67 \%$

4

$73.33 \%$

Q102 Which symbol represents a collection unit?

Answered: 15 Skipped: 14 
Symbol Set Survey - A Cartographic Representation and Modeling of Hopi

Archaeology Sites

\begin{tabular}{|c|c|c|}
\hline Symbol A & $93.33 \%$ & 14 \\
\hline Symbol B & $6.67 \%$ & 1 \\
\hline Total & & 15 \\
\hline
\end{tabular}

\section{Q103 Which symbol represents bedrock?}

Answered: 16 Skipped: 13

\begin{tabular}{|l|l|}
\hline Answer Choices & Responses \\
\hline \multicolumn{1}{|c|}{ Symbol A } & $0.00 \%$ \\
\hline Symbol B & $100.00 \%$ \\
\hline Total & \\
\hline & Q104 Which symbol represents a pithouse?
\end{tabular}

Answered: 15 Skipped: 14

Answer Choices

Symbol A

Symbol B
Responses

$6.67 \%$

$93.33 \%$

16

Q105 How easy was it to interpret the symbols to what they represent?

Answered: 16 Skipped: 13

\begin{tabular}{|l|l|}
\hline Answer Choices & Responses \\
\hline Easy & $0.00 \%$ \\
\hline Fair & $50.00 \%$ \\
\hline Moderate & $31.25 \%$ \\
\hline Difficult & $18.75 \%$ \\
\hline Challenging & $0.00 \%$ \\
\hline Total & \\
\hline
\end{tabular}

\section{Q106 How cohesive was the symbol set?}

Answered: 16 Skipped: 13

Answer Choices

Very cohesive

Fairly cohesive

Somewhat cohesive

Not cohesive

\begin{tabular}{lc}
\hline Responses & \\
\hline $\mathbf{1 2 . 5 0} \%$ & 2 \\
\hline $\mathbf{5 6 . 2 5 \%}$ & 9 \\
\hline $\mathbf{2 5 . 0 0 \%}$ & 4 \\
\hline $\mathbf{6 . 2 5 \%}$ & 1 \\
\hline
\end{tabular}


Symbol Set Survey - A Cartographic Representation and Modeling of Hopi

Archaeology Sites

\section{Q107 How sure are you about your answers?}

Answered: 15 Skipped: 14

\begin{tabular}{|c|c|c|}
\hline Answer Choices & \multicolumn{2}{|c|}{ Responses } \\
\hline Very sure & $6.67 \%$ & 1 \\
\hline Sure & $13.33 \%$ & 2 \\
\hline Fairly Sure & $66.67 \%$ & 10 \\
\hline Unsure & $13.33 \%$ & 2 \\
\hline Very unsure & $0.00 \%$ & 0 \\
\hline Total & & 15 \\
\hline
\end{tabular}

Q108 Is there any commentary you would

like to provided about Symbol Set 2?

Answered: 5 Skipped: 24

\section{Q109 Please, enter any additional comments you have below:}




\section{Appendix D. Python Script}

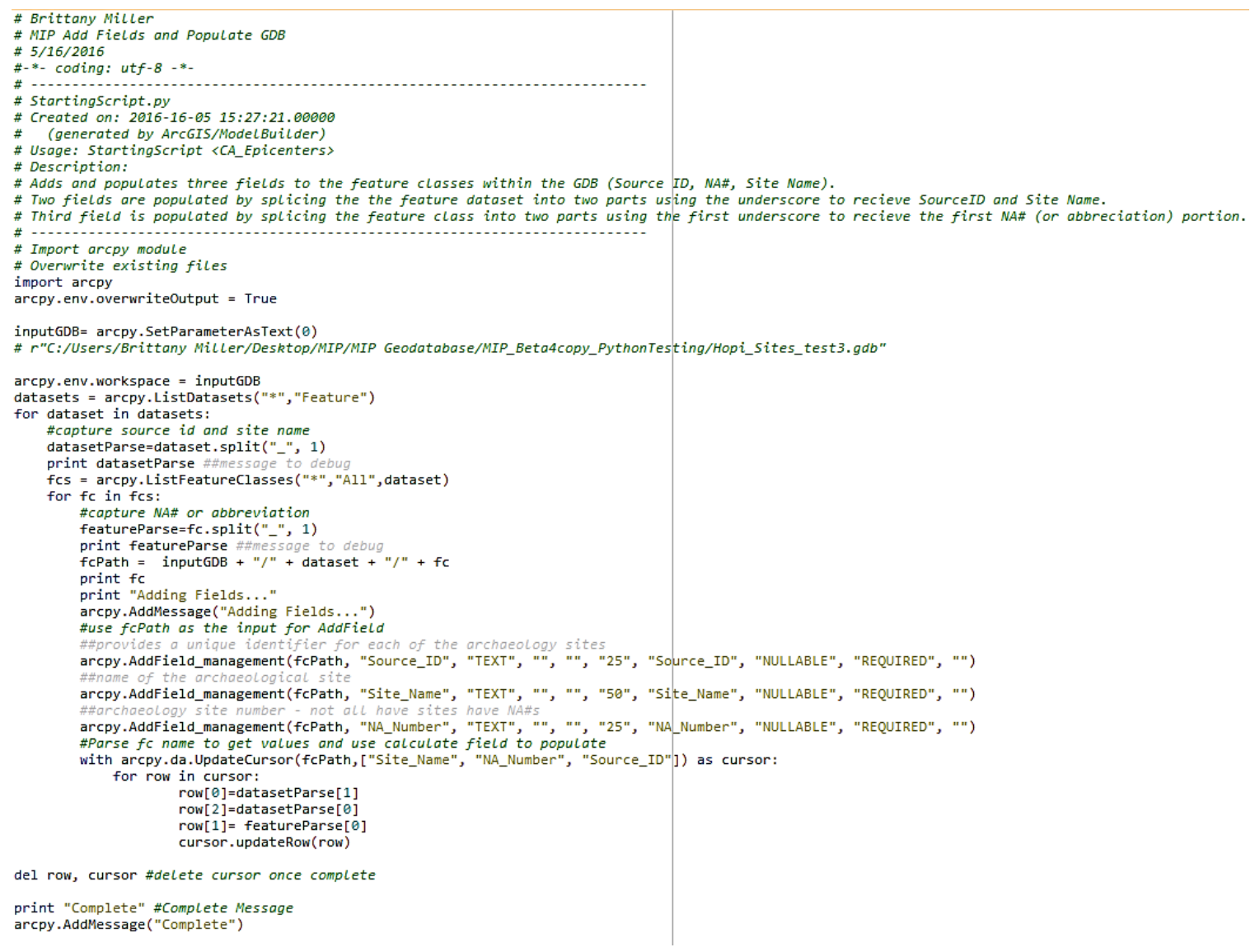

